Länderberichte 


\title{
Wahl und Wettbewerb im regulierten System - Normative Steuerung der Krankenhausversorgung in Deutschland
}

\author{
Markus Sichert*
}

Abkürzungen

Auswahl wichtiger (Reform-)Gesetze und Verordnungen für das Gesundheitswesen 67

I. Grundlagen

1. Der institutionelle Rahmen

1.1. Gesetzliche und Private Krankenversicherung

1.2. Finanzierungsstrukturen und solidarischer Ausgleich in der GKV bis 2008

1.3. Das GKV-Wettbewerbsstärkungsgesetz

1.3.1. Organisationsstrukturen und Wettbewerbsgrundlagen

1.3.2. Insbesondere: Neuordnung der Finanzierungsgrundlagen

1.4. Weiterentwicklung der Organisationsstrukturen:

Insolvenzfähigkeit auch der gesetzlichen Krankenkassen

2. Kernfragen des Wettbewerbs und der Regulierung im Gesundheitswesen

2.1. Die Rolle der Krankenhausversorgung

2.1.1. Die Krankenhausbehandlung und ihre Formen

2.1.2. Zur Relation zwischen ambulanten und stationären Behandlungsformen

2.1.3. Das Krankenhaus im Blickpunkt neuer Versorgungs- und Kooperationsformen

2.1.3.1. Das Krankenhaus - herausragender Akteur unter den Leistungserbringern

2.1.3.2. Integrierte Versorgung (IV)

2.1.3.3. Medizinische Versorgungszentren

2.1.4. Das Vordringen der Krankenhäuser in den ambulanten Bereich als Ausdruck des Wettbewerbs um den Erwerb von Krankenhäusern

2.1.5. Wettbewerb und Vergütungsstrukturen

2.1.5.1. Leistungsorientierung der Vergütung

2.1.5.2. Investitionskosten als Vergütungsbestandteil 94

2.1.6. Wettbewerb und Planung

2.1.7. Wettbewerb der Trägerstrukturen und Vertragswettbewerb 97

2.1.8. Qualität und Wettbewerb

2.2. Kostenbegrenzung und -kontrolle

2.2.2. Leistungsausgaben für die Krankenhausbehandlung in der PKV

2.2.3. Kosten der Krankenhäuser

2.2.4. Beteiligung der Krankenhäuser an finanziellen Stabilisierungsmaßnahmen

\footnotetext{
* Der Beitrag gibt ausschließlich die persönliche Auffassung des Verfassers wieder.
} 
2.2.4.1. Rechnungskürzungen mit Finanzierungsfunktion (Anschubfinanzierung IV)

2.2.4.2. „Sanierungsbeitrag“ der Krankenhäuser

2.2.5. Investitionen

2.3. Normative Steuerung und die politische Diskussion

2.3.1. Normative Steuerung

2.3.1.1. Normative und administrative Steuerung

2.3.1.2. Steuerung durch den formellen Gesetzgeber (Verantwortungsträger)

2.3.1.3. Doppelstellung der Länder als Adressaten und Verantwortungsträger

2.3.1.4. Steuerungsziele

2.3.1.5. Adressaten (I) - individuelle Leistungserbringer und Kostenträger

2.3.1.6. Adressaten (II) - (Spitzen-)Verbände und korporatistische Steuerung

2.3.1.7. Steuerungsansätze und -instrumente

2.3.1.8. Steuerungsaufwand und -anreize; Datenrelevanz

2.3.2. Akzente politischer Diskussion um die Krankenhausversorgung

3. Die Krankenhausversorgung

3.1. Der „Krankenhaussektor”

3.2. Organisationsstruktur des Krankenhaussektors

3.2.1. Determinanten

3.2.2. Zahl, Typologie und Organisation der Krankenhäuser

3.2.2.1. Trägerschaft

114

114

115

115

115

3.2.2.2. Krankenhaustypen und -arten

3.2.2.3. Unterscheidung nach Art der Zulassung

3.2.3. Aufgestellte Betten und Patientenbewegung

3.2.4. Ärztliches und nichtärztliches Personal

3.3. Historische Entwicklung

3.3.1. Funktionswandel der Krankenhäuser

3.3.2. Historische Entwicklung der Krankenhausfinanzierung

3.4.1. Allgemeines

3.4.2.1. Grundzüge

3.4.2.2. Aktuelle Entwicklungen im Zuge des KHRG 129

3.4.3. Krankenhausplanungsrecht

3.4.4. Leistungsrecht und Leistungserbringungsrecht 132

3.5 Kostenträger

4.1. Die Idee des Wettbewerbs

4.3. Verfassungsrechtliche Fundierung

4.3.1. Verfassungsrechtlicher Schutz der Formen freier wirtschaftlicher Betätigung

4.3.2. Grundrechtlicher Maßstab für selektivvertragliches Kontrahieren

4.3.3. Eigentumsgarantie 
4.4. Wettbewerbsrechtlicher Rahmen

4.4.1. Allgemeine Grundsätze der Anwendbarkeit des Wettbewerbsrechts, v.a. im Verhältnis der Krankenkassen zu den Leistungserbringern

4.4.2. Zusammenwirken von nationalem und europäischem Recht

4.4.3. Wettbewerbsrecht

4.4.3.1. Gesetz gegen den unlauteren Wettbewerb (UWG) und Heilmittelwerbegesetz (HWG)

4.4.3.2. EG-Beihilfenrecht

4.4.4. Kartellrecht

4.4.4.1. Nationales Kartellrecht

4.4.4.2. Europäisches Kartellrecht

4.4.5 Vergaberecht

4.4.6. Berufsrecht

4.5. Kontrollbehörden

4.5.1. Nationale Kartellbehörden

4.5.2. Europäische Kommission

4.5.2.1. Kartellverfahren

4.5.2.2. Prüfung der Vereinbarkeit von Beihilfen mit Gemeinschaftsrecht 159

4.5.2.3. Durchführung vergaberechtlicher Bestimmungen 160

4.5.3. Aufsichtsbehörden der Krankenkassen

4.5.4. Zuständigkeiten im vergaberechtlichen Nachprüfungsverfahren gem. GWB

II. Wettbewerb und Regulierung in der Krankenhausversorgung

1. Verbraucherorientierte Anreize der Krankenhausauswahl

1.1. Gesetzliche Krankenversicherung

1.1.1. Das Recht zur Wahl des Krankenhauses

1.1.2. Die Ausübung des Wahlrechts

1.1.3. Die Qualität der Versorgung und Qualitätsberichte als maßgebliche Faktoren der Auswahlentscheidung

1.1.4. Einschränkung des Rechts der Krankenhauswahl durch „Managed Care“

1.1.5. Werbung durch Krankenhäuser

1.2. Besonderheiten im Recht der PKV

$\begin{array}{ll}\text { 2. Die Wahl der Versicherung durch den Verbraucher } & 176 \\ \text { 2. Gesetzliche Krankenversicherung } & 176\end{array}$

2.1.1. Wahl- und Wechselrechte

2.1.2. Wahl der Krankenkasse aufgrund des Angebots krankenhausspezifischer Versorgungskonzepte (IV, DMP)

2.1.3. Wahl der Krankenkasse aufgrund tarifärer Privilegierung der Nichtinanspruchnahme stationärer Leistungen?

2.1.4. Die Vermittlung privater Zusatzversicherungen für die Krankenhausbehandlung seitens der Krankenkassen

2.1.5. Mitgliederwerbung

2.1.6. Wahltarif „Kostenerstattung für Chefarztbehandlung bzw. Ein-/ Zweibettzimmer"?

2.2. Besonderheiten im Recht der PKV

3.1. Gesetzliche Krankenversicherung

3.1.1. Das Grundverhältnis zwischen Krankenkassen und Krankenhäusern

3.1.2. Besonderheiten im ambulanten Bereich 
3.1.3. „Regulierungsverflechtung“

3.1.4. Vertragswettbewerb

3.1.4.1. Allgemeines

3.1.4.2. „DMP-Verträge“"

3.1.4.3. Verträge zur Integrierten Versorgung 189

3.1.5. Die Krankenkassen als Unternehmen

3.1.6. Ein neuer ,bestimmter Markt“ innerhalb des GKV-Systems? 196

3.1.7. Pflicht zur Ausschreibung von Kooperationsverträgen 197

3.2. Besonderheiten im Recht der PKV 198

4. Wettbewerb zwischen Krankenhäusern 199

4.1. Markteintritt; Konkurrenz um die (Plan-)Position im regulierten System 200

4.2. Angebotswettbewerb und Preiswettbewerb 202

4.2.1. Preisgestaltung nach Art der Leistung 202

4.2.2. Qualität, Versorgungsangebot und Spezialisierung 204

4.2.3. Absatzmenge 206

4.2.4. Exkurs: Einflüsse veränderten Kodierverhaltens 206

4.2.5. Besondere Versorgungsformen: Konzeption und Haftung 208

4.3. Einweisungssteuerung seitens der Krankenhäuser 209

4.3.1. Gründung von und Steuerung durch MVZ 209

4.3.2. „Einweisungspauschalen“ an Ärzte 211

4.3.2.1. Die Problematik 211

4.3.2.2. Sozialrechtliches Selektivvertragsrecht versus Berufsrecht? 213

4.3.3. Vereinbarungen/Absprachen zwischen den Krankenhäusern 214

4.4. Der Wettbewerb um Kooperationsgemeinschaften:

Die „Binnenkooperation“ mit anderen Leistungserbringern (,VorabIntegration")

4.5. Verdrängungswettbewerb: Konzentrationsprozeß und Gemeinwohlaufgaben

4.5.1. Entwicklungstendenzen $\quad 216$

$\begin{array}{ll}\text { 4.5.2. Verlustausgleich } & 217\end{array}$

4.6. Fusionswettbewerb $\quad 219$

4.7. Wettbewerbsdruck und strukturelle Reorganisation 220

4.7.1. Allgemeine Tendenzen, Zentrenbildung und Bedeutung für die Notfallversorgung

4.7.2. Symbiose von Plankrankenhaus und gewerblicher Privatklinik 222

4.7.3. Case- und Personalmanagement 224

4.7.4. Grunddaten der Krankenhäuser: Indikatoren struktureller Reorganisation?

$\begin{array}{ll}\text { 5. Wettbewerb zwischen den Versicherungsträgern } & 227 \\ \text { 5.1. Gesetzliche Krankenkassen als Konkurrenten im Wettbewerb } & 227\end{array}$

5.1.1. Exkurs: Der Risikostrukturausgleich (RSA) alter Prägung 228

5.1.2. Interner Steuerungsmechanismus: Neuordnung der

Finanzierungsströme im Zeichen des Gesundheitsfonds

5.1.2.1. (Risikoadjustierte) Zuweisungen aus dem Gesundheitsfonds 229

5.1.2.2. Kassenindividuelle Finanzierungsinstrumente (Zusatzbeiträge, Prämien)

5.1.3. Externer Kontrollmechanismus: Zur Anwendung des Wettbewerbs- und Kartellrechts

5.1.3.1. Allgemeines und Mitgliederwerbung 233

5.1.3.2. Fusionskontrolle 
5.1.4. „Ausschluss vom Markt": Schließung und Insolvenz

5.2. Wettbewerb zwischen privaten Versicherungsunternehmen

5.3. Wettbewerb zwischen gesetzlichen Krankenkassen und PKV-Unternehmen

III. Wesentliche Ergebnisse - Wahl und Wettbewerb im regulierten System

1. Zur Einführung und Kontrolle marktförmiger Strukturen durch Recht

2. Steuerungseffekte und Interdependenzen

2.1. Finanzierung, Ausgaben und Effizienz

2.2. Zusammenwirken und Wechselwirkungen: „Regulierungsverflechtung“

2.3. Wettbewerbsverzerrungen und exogene Stützung

3. Fazit

Literatur 


\section{Abkürzungen}

A.a.O.

AEV

a.F.

Abs.

AfG

AMPreisV

AOK

ApoG

ApoR

A\&R

Art.

Aufl.

AVWG

Az.

BAnz.

BayKrG

BayGVBl.

Bd.

Beck-OK

Bek.

Beschl.

BGB

BGB1.

BGH

BKartA

BKK

BMAS

BMG

BMGS

$\mathrm{BMV}(\ddot{\mathrm{A}})$

BPflV

BR-Drucks

BSG

BSGE

bspw.

BSSichG

BStatG

BT-Drucks.

BVA

BVerfG

BVerfGE

BVerfGK

BVerwG

bzw.

ca.

CDU
An angegebenem Ort

Arbeiter-Ersatzkassen-Verband e.V.

(m.W. zum 31.12.2008 aufgelöst, s. vdek)

alte(r) Fassung

Absatz

Ausschuss für Gesundheit

Arzneimittelpreisverordnung

Allgemeine Ortskrankenkasse(n)

Gesetz über das Apothekenwesen (Apothekengesetz)

Apotheke und Recht (Zeitschrift)

Apotheke und Recht (Zeitschrift)

Artikel

Auflage

s. Auswahl wichtiger (Reform-)Gesetze

Aktenzeichen

Bundesanzeiger

Bayerisches Krankenhausgesetz

Bayerisches Gesetz- und Verordnungsblatt

Band

Beckscher Online-Kommentar (Sozialrecht)

Bekanntmachung

Beschluß

Bürgerliches Gesetzbuch

Bundesgesetzblatt

Bundesgerichtshof

Bundeskartellamt

Betriebskrankenkasse

Bundesministerium für Arbeit und Soziales

Bundesministerium für Gesundheit

(vormals) Bundesministerium für Gesundheit und soziale Sicherung

Bundesmantelvertrag - Ärzte

Verordnung zur Regelung der Krankenhauspflegesätze

(Bundespflegesatzverordnung)

Bundesrats-Drucksache

Bundessozialgericht

Entscheidungen des Bundessozialgerichts (amtliche Sammlung)

beispielsweise

s. Auswahl wichtiger (Reform-)Gesetze

Bundesstatistikgesetz

Bundestags-Drucksache

Bundesversicherungsamt

Bundesverfassungsgericht

Entscheidungen des Bundesverfassungsgerichts (amtliche Sammlung)

Kammerentscheidungen des Bundesverfassungsgerichts

Bundesverwaltungsgericht

beziehungsweise

circa

Christlich Demokratische Union Deutschlands 
CSU

DÄ

DAWI

ders.

d.h.

dies.

DIMDI

DKG

DKI

DMP

DRG

DRV KBS

EBM

EG

EGV

EJSS

endg.

Einl.

ErsK

EuG

$\mathrm{EuGH}$

EuR

EuZW

EWG

f., ff.

Fn.

FPÄndG

FPG

FPV

G

GA

GBA

gem.

GesR

Gew

GewArch

GG

ggf.

GKV

GKV-GRG

GKV-OrgWG

GKV-WSG

$\mathrm{gGmbH}$

GMG

$\mathrm{GmbH}$

GOÄ

GRG

GRUR

GSG

GuS

GWB
Christlich Soziale Union

Deutsches Ärzteblatt

Dienstleistungen von allgemeinem wirtschaftlichen Interesse

derselbe, denselben

das heißt

dieselbe, dieselben

Deutsches Institut für medizinische Dokumentation und Information

Deutsche Krankenhausgesellschaft

Deutsches Krankenhausinstitut

Disease Management Programm

Diagnosis Related Groups

Deutsche Rentenversicherung Knappschaft Bahn See

Einheitlicher Bewertungsmaßstab

Europäische Gemeinschaft

Vertrag zur Gründung der Europäischen Gemeinschaft

European Journal of Social Security

endgültig

Einleitung

Die Ersatzkasse (Zeitschrift)

Europäisches Gericht erster Instanz

Europäischer Gerichtshof

Europarecht (Zeitschrift)

Zeitschrift für Europäisches Wirtschaftsrecht

Europäische Wirtschaftsgemeinschaft

folgende

Fußnote

s. Auswahl wichtiger (Reform-)Gesetze

s. Auswahl wichtiger (Reform-)Gesetze

Fallpauschalenvereinbarung

Gesetz

Generalanwalt

Gemeinsamer Bundesausschuß

gemäß

Gesundheitsrecht (Zeitschrift)

Gewerbeordnung

Gewerbearchiv (Zeitschrift)

Grundgesetz

gegebenenfalls

Gesetzliche Krankenversicherung

s. Auswahl wichtiger (Reform-)Gesetze

s. Auswahl wichtiger (Reform-)Gesetze

s. Auswahl wichtiger (Reform-)Gesetze gemeinnützige $\mathrm{GmbH}$

s. Auswahl wichtiger (Reform-)Gesetze

Gesellschaft mit beschränkter Haftung

Gebührenordnung für Ärzte

s. Auswahl wichtiger (Reform-)Gesetze

Gewerblicher Rechtsschutz und Urheberrecht (Zeitschrift)

s. Auswahl wichtiger (Reform-)Gesetze

Gesundheits- und Sozialpolitik

Gesetz gegen Wettbewerbsbeschränkungen 


\begin{tabular}{|c|c|}
\hline Hrsg. & Herausgeber \\
\hline HWG & Gesetz über die Werbung auf dem Gebiet des Heilwesens \\
\hline HS & Halbsatz \\
\hline i.d.F. & in der Fassung \\
\hline i.H.v. & in Höhe von \\
\hline InEK & Institut für das Entgeltsystem im Krankenhaus \\
\hline $\mathrm{InsO}$ & Insolvenzordnung \\
\hline IV & Integrierte Versorgung \\
\hline i.V.m. & in Verbindung mit \\
\hline $\mathrm{Jh}$. & Jahrhundert \\
\hline $\mathrm{JZ}$ & Juristenzeitung \\
\hline Kap. & Kapitel \\
\hline KBV & Kassenärztliche Bundesvereinigung \\
\hline KG & Kommanditgesellschaft \\
\hline KHG & s. Auswahl wichtiger (Reform-)Gesetze \\
\hline KHEntgG & s. Auswahl wichtiger (Reform-)Gesetze \\
\hline KHStatV & Krankenhausstatistikverordnung \\
\hline KHuR & Krankenhaus \& Recht (Zeitschrift) \\
\hline KHR & Zeitschrift für das gesamte Krankenhausrecht \\
\hline KHRG & s. Auswahl wichtiger (Reform-)Gesetze \\
\hline KG & Kommanditgesellschaft \\
\hline KOM & Kommission der Europäischen Gemeinschaften \\
\hline KV & Kassenärztliche Vereinigung, Krankenversicherung \\
\hline $\mathrm{KrV}$ & Die Krankenversicherung (Zeitschrift) \\
\hline LG & Landgericht \\
\hline LS & Leitsatz \\
\hline lt. & laut \\
\hline m. Änd. & mit Änderungen \\
\hline $\mathrm{MB} / \mathrm{KK}$ & $\begin{array}{l}\text { Allgemeine Versicherungsbedingungen für die Krankheitskosten- und } \\
\text { Krankenhaustagegeldversicherung/Teil I - Musterbedingungen des } \\
\text { Verbandes der privaten Krankenversicherung }\end{array}$ \\
\hline MBO & Musterberufsordnung für die deutschen Ärztinnen und Ärzte \\
\hline MedR & Medizinrecht (Zeitschrift) \\
\hline Mio. & Millionen \\
\hline MMG & Managementgesellschaft \\
\hline Mrd. & Milliarden \\
\hline MVZ & Medizinisches Versorgungszentrum \\
\hline m.w.N. & mit weiteren Nachweisen \\
\hline N. & Nachweis(e) \\
\hline n.F. & neue(r) Fassung \\
\hline NJW & Neue Juristische Wochenschrift \\
\hline NJW-RR & NJW-Rechtsprechungsreport (Zeitschrift) \\
\hline $\mathrm{Nr}$ & Nummer(n) \\
\hline NVwZ & Neue Zeitschrift für Verwaltungsrecht \\
\hline NZS & Neue Zeitschrift für Sozialrecht \\
\hline o.ä. & oder ähnliches \\
\hline OLG & Oberlandesgericht \\
\hline p.a. & per annum \\
\hline PflWeitG & s. Auswahl wichtiger (Reform-)Gesetze \\
\hline PharmaR & Pharma-Recht (Zeitschrift) \\
\hline PharmR & Pharma-Recht (Zeitschrift) \\
\hline
\end{tabular}




\begin{tabular}{|c|c|}
\hline PKV & Private Krankenversicherung \\
\hline PM & Pressemitteilung \\
\hline PPP & Public Private Partnership \\
\hline PrOVGE & Entscheidungen des Preußischen Oberverwaltungsgerichts \\
\hline Q & Quartal \\
\hline rd. & rund \\
\hline RGBl. & Reichsgesetzblatt \\
\hline $\mathrm{RL}$ & Richtlinie \\
\hline Rn. & Randnummer(n) \\
\hline Rs.(en) & Rechtssache(n) \\
\hline RSA & Risikostrukturausgleich \\
\hline RSAV & s. Auswahl wichtiger (Reform-)Gesetze \\
\hline RWI & Rheinisch-Westfälisches Institut für Wirtschaftsforschung \\
\hline s., S. & siehe, Seite(n), Satz \\
\hline SF & Sozialer Fortschritt (Zeitschrift) \\
\hline SGB II & $\begin{array}{l}\text { Sozialgesetzbuch (SGB) - Zweites Buch (II) - Grundsicherung für } \\
\text { Arbeitssuchende }\end{array}$ \\
\hline SGB IV & $\begin{array}{l}\text { Sozialgesetzbuch (SGB) - Viertes Buch (IV) - Gemeinsame Vorschriften } \\
\text { für die Sozialversicherung }\end{array}$ \\
\hline SGB V & s. Auswahl wichtiger (Reform-)Gesetze \\
\hline SGB $\mathrm{X}$ & $\begin{array}{l}\text { Sozialgesetzbuch (SGB) - Zehntes Buch (X) } \\
\text { - Sozialverwaltungsverfahren und Sozialdatenschutz }\end{array}$ \\
\hline SGB XI & Sozialgesetzbuch (SGB) - Elftes Buch (XI) - Soziale Pflegeversicherung \\
\hline SGB XII & Sozialgesetzbuch (SGB) - Zwölftes Buch (XII) - Sozialhilfe \\
\hline SGG & Sozialgerichtsgesetz \\
\hline SozSich & Soziale Sicherheit \\
\hline SPD & Sozialdemokratische Partei Deutschlands \\
\hline StBA & Statistisches Bundesamt \\
\hline SVHV & Verordnung über das Haushaltswesen in der Sozialversicherung \\
\hline tw. & teilweise \\
\hline u.a. & unter anderem, unter anderen \\
\hline UAbs. & Unterabsatz \\
\hline u.a.m. & und anderes mehr \\
\hline Urt. & Urteil \\
\hline u.U. & unter Umständen \\
\hline UWG & Gesetz gegen den unlauteren Wettbewerb \\
\hline v. & von, vom \\
\hline VAG & Versicherungsvertragsgesetz \\
\hline VÄndG & s. Auswahl wichtiger (Reform-)Gesetze \\
\hline vdek & Verband der Ersatzkassen e.V. \\
\hline verb. & verbunden \\
\hline VersR & Versicherungsrecht (Zeitschrift) \\
\hline vgl. & vergleiche \\
\hline $\mathrm{VgV}$ & Verordnung über die Vergabe öffentlicher Aufträge - Vergabeverordnung \\
\hline VO & Verordnung \\
\hline VOF & Verdingungsordnung für freiberufliche Leistungen \\
\hline Vol. & Volume \\
\hline $\mathrm{VOL} / \mathrm{A}$ & Verdingungsordnung für Leistungen - Teil A \\
\hline VSSR & Vierteljahresschrift für Sozialrecht (Zeitschrift) \\
\hline VVG & Versicherungsaufsichtsgesetz \\
\hline zahlr. & zahlreich \\
\hline
\end{tabular}


Markus Sichert

z.B.

Ziff.

zit.

ZMGR

ZuInvG zum Beispiel

Ziffer(n)

zitiert

Zeitschrift für das gesamte Medizin- und Gesundheitsrecht Gesetz zur Umsetzung der Zukunftsinvestitionen der Kommunen und Länder (Zukunftsinvestitionsgesetz - ZuInvG) 


\section{Auswahl wichtiger (Reform-)Gesetze und Verordnungen für das Gesundheitswesen}

$\begin{array}{lll}\text { G. v. 20.12.1988 } & \text { SGB V } & \begin{array}{l}\text { Sozialgesetzbuch (SGB) Fünftes Buch (V) } \\ \text { - Gesetzliche Krankenversicherung }\end{array} \\ \text { G. v. 20.12.1988 GRG } & \begin{array}{l}\text { Gesetz zur Strukturreform im Gesund- } \\ \text { heitswesen (Gesundheits-Reformgesetz - } \\ \text { GRG) }\end{array}\end{array}$

BGB1. I S. 2477

Bek.v.

KHG

Gesetz zur wirtschaftlichen Sicherung der

10.4.1991

Krankenhäuser und zur Regelung der

Krankenhauspflegesätze (Krankenhausfinanzierungsgesetz - KHG)

G. v. 21.12.1992 [GSG]

V. 3.1 .1994

RSAV

v. 26.9.1994

BPflV

G. v. 22.1.1999

[GKVGRG]

G. v. 23.4.2002 KHEntgG
Gesetz zur Sicherung und Strukturverbesserung der gesetzlichen Krankenversicherung (Gesundheitsstrukturgesetz)

Verordnung über das Verfahren zum Risikostrukturausgleich in der gesetzlichen Krankenversicherung (RisikostrukturAusgleichsverordnung - RSAV)

Verordnung zur Regelung der Krankenhauspflegesätze (Bundespflegesatzordnung - BPflV)

Gesetz zur Reform der gesetzlichen Krankenversicherung ab dem Jahr 2000 (GKVGesundheitsreformgesetz 2000)

Gesetz über die Entgelte für voll- und teilstationäre Krankenhausleistungen (Krankenhausentgeltgesetz - KHEntgG)

Gesetz zur Einführung des diagnoseorientierten Fallpauschalensystems für Krankenhäuser (Fallpauschalengesetz FPG)

G. v. 23.12.2002 BSSichG Gesetz zur Sicherung der Beitragssätze in
BGB1. I

S. 1412, 1422

BGB1. I S. 1412

BGB1. I S. 2477

BGB1. I S. 886

BGBl. I S. 2266

BGB1. I S. 55

BGB1. I S. 2750

BGB1. I S. 2626

BGB1. I S. 4637

1 Verkündet als Art. 1 GRG. 
der gesetzlichen Krankenversicherung und in der gesetzlichen Rentenversicherung (Beitragssatzsicherungsgesetz - BSSichG)
G. v. 17.7.2003
FPÄndG

Gesetz zur Änderung der Vorschriften zum diagnose-orientierten Fallpauschalensystem für Krankenhäuser (Fallpauschalenänderungsgesetz - FPÄndG)
G. v.
14.11.2003

GMG

Gesetz zur Modernisierung der gesetzlichen Krankenversicherung (GKVModernisierungsgesetz - GMG)

BGB1. I S. 1461

BGBl. I S. 2190, geänd. durch G. v. 15.12.2004, BGB1. I S. 3445

BGB1. I S. 3429

G. v.

15.12.2004

2.FPÄndG

Zweites Gesetz zur Änderung der Vorschriften zum diagnose-orientierten Fallpauschalensystem für Krankenhäuser und zur Änderung anderer Vorschriften (Zweites Fallpauschalenänderungsgesetz - 2 . FPÄndG)

G. v. 26.4.2006

[AVWG]

Gesetz zur Verbesserung der Wirtschaftlichkeit in der Arzneimittelversorgung

G. v.

VÄndG

22.12.2006

G. v. 26.3.2007

GKV-WSG

Gesetz zur Änderung des Vertragsarztrechts und anderer Gesetze (Vertragsarztrechtsänderungsgesetz - VÄndG)

BGB1. I S. 984

BGBl. I S. 3439

BGB1. I S. 378 der gesetzlichen Krankenversicherung (GKV-Wettbewerbsstärkungsgesetz GKV-WSG)

G v. 28.5.2008 [PflWeitG] Gesetz zur strukturellen Weiterentwicklung der Pflegeversicherung (PflegeWeiterentwicklungsgesetz)

G. v. 15.12.2008

GKVOrgWG

Gesetz zur Weiterentwicklung der Organisationsstrukturen in der gesetzlichen Krankenversicherung (GKV-OrgWG)

G. v. 17.3.2009 KHRG
BGBL. I S. 874

BGBl. I S. 1426

BGB1. I S. 534
Gesetz zum ordnungspolitischen Rahmen der Krankenhausfinanzierung ab dem Jahr 2009 (Krankenhausfinanzierungsreformgesetz - KHRG) 


\section{Grundlagen}

Wahlmöglichkeiten dominieren im Wettbewerb, und „Wettbewerb“ dominiert die Wortwahl gesundheitspolitischer Reformvorhaben der jüngeren Zeit. Die ,wettbewerbliche und freiheitliche Ausrichtung des Gesundheitswesens" war das zentrale Postulat zur Gesundheitspolitik seitens der koalierenden Parteien eingangs der 16. Wahlperiode (2005-2009), ${ }^{2}$ in der die v.a. seit dem GKV-Modernisierungsgesetz (GMG, $2003)^{3}$ so genannte ,solidarische Wettbewerbsordnung" fortentwickelt wurde.

Den Auftakt der Reformvorhaben ${ }^{4}$ bildete das Gesetz zur Verbesserung der Wirtschaftlichkeit in der Arzneimittelversorgung (AVWG) mit dem Ziel, den „Preiswettbewerb zu beleben“. Änderungen des Vertragsarztrechts und anderer Gesetze (VÄndG) dienten der Beseitigung von Wettbewerbshindernissen, um „die vertragsärztliche Berufsausübung effizienter und damit wettbewerbsfähiger zu gestalten“. 5 Verstärkt ,,wettbewerblich ausgerichtet" wurde das Gesundheitssystem alsdann durch das überwiegend zum 1.4.2007, 1.7.2008 und 1.1.2009 in Kraft getretene „Wettbewerbsstärkungsgesetz“ (GKV-WSG). Es verfolgt einen „Wettbewerb um Qualität und Wirtschaftlichkeit“6 und offeriert „mehr Wahlmöglichkeiten und neue Leistungen für GKV-Versicherte"7 ebenso wie „mehr Wettbewerb der Leistungserbringer durch größere Vertragsfreiheit für Krankenkassen"8 Das Gesetz zur Weiterentwicklung der Organisationsstrukturen in der gesetzlichen Krankenversicherung (GKV-OrgWG) schließlich konkretisiert diese Ausrichtung und „steuert nach“, indem vor dem Hintergrund des bis dahin ungleichen Insolvenzrechts für bundesunmittelbare Kassen einerseits und landesunmittelbare andererseits die „ungleichen wettbewerblichen Ausgangspositionen“ beseitigt und die Regelungen über die Haftung bei Schließung einer Krankenkasse im Hinblick auf die ,wettbewerblichen Strukturen des GKV-Systems" angepasst werden sollen. ${ }^{9}$

Diese Begriffsdominanz sagt nicht zwingend etwas darüber aus, welches primäre Ziel der Gesetzgeber bzw. mit welcher Intensität er dieses verfolgt. Betreibt er die „Stärkung des Wettbewerbs“ um der korrespondierenden Freiheit der Akteure willen oder dient diese zuvorderst der Instrumentalisierung zur effizienten Versorgung in Diensten der Solidargemeinschaft? Es steht zu vermuten, daß die Antwort - wenngleich in unterschiedlicher Gewichtung - beide Aspekte in sich vereint; v.a. ist offensichtlich,

2 Koalitionsvertrag zwischen CDU, CSU und SPD vom 11.1.2005, www.cducsu.de/upload/koavertrag0509.pdf, B IV. 7.2.2., S. 103.

3 Vgl. BT-Drs. 15/1525, S. 1, 71.

4 Vgl. die Übersicht „Auswahl wichtiger (Reform-)Gesetze für das Gesundheitswesen“.

5 BT-Drs. 16/2474, S. 1.

6 Vgl. BT-Drucks. 16/3100, S. 85.

7 A.a.O., S. 85.

8 A.a.O., S. 86.

9 BT-Drs. 16/9559, S. 1. 
daß sich Wettbewerb seinerseits in normativ beschriebenen Bahnen vollzieht. ${ }^{10}$ Danach gilt es, Instrumentarien, Kausalitäten und Potenziale zu identifizieren und ggf. zu bewerten. Das AVWG markiert offen das Spannungsfeld zwischen Wettbewerb und (Preis-)Regulierung, ${ }^{11}$ und der im GKV-WSG verfolgte Ansatz eines Wettbewerbs starker Marktteilnehmer geht einher mit einer - zentralisierenden - Straffung von Organisationsstrukturen, wie etwa der Bündelung von Kompetenzen beim neu gebildeten Spitzenverband Bund der Krankenkassen, sowie mit der konzentrierten Neuordnung der Finanzströme.

Wendet man den Blick nunmehr auf die im selben Zeitraum markanten äußeren Fakten, so fällt auf, daß die Ausgaben für die stationäre Versorgung in der gesetzlichen Krankenversicherung 2006 erstmals die Marke von $€ 50$ Mrd. übersteigen und im Jahr darauf erstmals mehr als $€ 1.000$ je Mitglied betragen. ${ }^{12}$ Zugleich setzt sich der Konzentrationsprozess auf Seiten der Kostenträger und Leistungserbringer (Krankenhäuser) fort: Nach der amtlichen Statistik KM1 existierten im Januar 2009 noch 202 gesetzliche Krankenkassen. ${ }^{13}$ Aus dem zum 1. Januar 2009 gestarteten Gesundheitsfonds erhielten genau 193 Krankenkassen ${ }^{14}$ Zuweisungen zur Deckung ihrer Ausgaben. Gegen Ende der Wahlperiode schließlich näherte sich die kontinuierlich sinkende Zahl der Krankenhäuser der Marke von 2.000 Einrichtungen.

Als größter Ausgabenposten nicht nur der gesetzlichen, sondern auch der privaten KV rückt die Krankenhausbehandlung naturgemäß in den Mittelpunkt, soweit der Reformbedarf - wie etwa im GKV-WSG - mit der Ineffizienz der verwendeten Mittel, aber auch dem nicht optimalen Einsatz der Ressourcen an Schnittstellen der Versorgung begründet wird. ${ }^{15} \mathrm{Zu}$ Beginn des Jahres 2009 attestierte eine Untersuchung des $R W I \mathrm{zu}$ „Effizienzreserven im Gesundheitswesen" 16 dem Krankenhaussektor bezogen auf das Jahr 2007 ein konservativ geschätztes Einsparpotenzial von $€ 2,19$ Mrd., das auf der Basis optimistischer Schätzungen gar $€ 4,37$ Mrd. betragen soll.

Dieses Szenario mutet zunächst seltsam an, führt man sich die Brisanz der wirtschaftlichen Situation der Krankenhäuser entsprechend den Umfragen 2007 und 2008 (Krankenhaus Barometer) des DKI vor Augen. ${ }^{17}$ Danach schrieben 28\% (Umfrage

10 Vgl. BGH, Beschl. v. 16.1.2008, KVG 26/07, NZS 2008, S. 653 (654): „Durch die staatliche Förderung und wirtschaftliche Planung des Krankenhauswesens wird in erheblichem Maße regulierend auf den Marktzutritt, die Marktbedingungen und die Marktentfaltung der Krankenhäuser Einfluss genommen.“.

11 Vgl. Sichert, Neue Versorgungsformen und Rabattregelungen für ausländische Leistungserbringer, EuR, Beiheft 2/2007, S. $101 \mathrm{ff}$.

12 Näher unten II.2.1.

13 BMG, Ergebnisse der GKV-Statistik KM1, Stand: 10. März 2009.

14 Die landwirtschaftlichen Krankenkassen nehmen am Risikostrukturausgleich nicht teil, § 266 Abs. 9 SGB V.

15 BT-Drs. 16/3100, S. 87.

16 Augurzky/Tauchmann/Werblow/Felder, Essen, 2009.

17 DKI (Hrsg.), Krankenhaus Barometer, Umfrage 2007; Umfrage 2008. 
2007) bzw. knapp 30\% (2008) der (304 bzw. 347 an der Umfrage beteiligten) Krankenhäuser Verluste. ${ }^{18}$ Der Umfrage 2007 zufolge befürchteten $42 \%$ der beteiligten Häuser eine Verschlechterung ihrer wirtschaftlichen Situation für das Jahr 2008 gegenüber 2007. ${ }^{19}$ Hinsichtlich der Kostendeckung im DRG-System meldete ein Viertel der Häuser, daß im Jahr 2006 eine Deckung nicht zu erzielen war, da in diesen Häusern zu viele der ihrer Ansicht nach nicht angemessen (relativ) bewerteten Leistungen erbracht wurden. ${ }^{20}$ Der Anteil der Krankenhäuser mit einem Jahresüberschuss war im Folgejahr wiederum rückläufig $(-3,5 \%){ }^{21}$

Die $D K G^{22}$ warnte, die Streichung von $€ 4$ Mrd. ginge angesichts der Personalkostenquote mit einer Streichung von ca. 60.000 Arbeitsplätzen einher, und mahnte im Kontext der Finanzkrise 2008/2009 zusätzliche Investitionen im Krankenhausbereich an. Die von der $D K G$ entsprechend in Auftrag gegebenen Prognos-Studie 23 attestiert solchen Investitionen besonders günstige makroökonomische Auswirkungen in Form einer zusätzlichen Bruttowertschöpfung von $0,85 \%$ BIP-Zuwachs bei $€ 10$ Mrd. Investitionsvolumen ${ }^{24}$ bzw. einem Multiplikator je investiertem Euro von 1,8.

Schon hier scheint eine Verflechtung politischer Ziele auf, auch wenn die vorgenannte Forderung nur zum Teil Eingang in das sog. „Konjunkturpaket II“ gefunden hatte: So wurden im Rahmen des Zukunftsinvestitionsgesetzes (ZuInvG) gem. § 3 Abs. 1 S. 1 Ziff. 2 lit. a) auch Krankenhäuser als „Investitionsschwerpunkte Infrastruktur“ anerkannt, die neben zahlreichen weiteren sowohl im Bereich der Bildungs- als auch der allgemeinen Infrastruktur mit insgesamt $€ 10$ Mrd. ( 1 Abs. 1 S. 2 ZuInvG) gefördert werden sollen. Überdies wurde die in Art. 14 des Gesetzes zur Sicherung von Beschäftigung und Stabilität in Deutschland verankerte Senkung des allgemeinen Beitragssatzes zur GKV 25 in den Dienst von Beschäftigung und Stabilität gestellt, wobei die Umsetzung durch die entlastungswirksam vorgezogene Erhöhung des Bundeszuschusses (Art. 13 des Gesetzes) nur bedingt mit der gesetzlichen Funktionsbindung, nämlich der ,pauschale(n) Abgeltung der Aufwendungen der Krankenkassen für versicherungsfremde Leistungen" 26 korreliert.

Ansätze zur Neuordnung des Krankenhausbereichs kreisen seit langem um Fragen einer Reform des Vergütungssystems und der Investitionskostenfinanzierung. Im lang-

18 A.a.O., S. 77 (Umfrage 2007), bzw. S. 62 (Umfrage 2008).

19 Umfrage 2007, S. 80.

20 A.a.O., S. 19.

21 Umfrage 2008, S. 62.

22 Pressemitteilung vom 8. Januar 2009 (www.dkgev.de).

23 Zusammenfassung unter www.dkgev.de; Zugriff: Jan. 2009.

24 S. auch unten I.2.2.5.

25 Vgl. Art. 13 und 14 des Gesetzes zur Sicherung von Beschäftigung und Stabilität in Deutschland vom 2. März 2009, BGBl. I 2009, S. 416 (431); vgl. zur Begründung noch BT-Drs. 16/11740, dort S. 1,33 .

26 Allgemein kritisch Rixen, in: Becker/Kingreen, SGB V 2008, § 221 Rn. 3 und 4 (,paradoxe Formulierung"). 
jährigen Streit um Verknüpfung und Interdependenz beider Aspekte stoßen die vehement verfochtenen Grundsatzpositionen immer wieder und oftmals unversöhnlich aufeinander. In der Reformdiskussion ringen zwei Konzepte um die Vorherrschaft: Die Umstellung zugunsten einer integrierten Abbildung und Finanzierung auch der Investitionskosten über die Leistungsvergütung (Monistik) einerseits und Ansätze zur modifizierten Fortentwicklung der externen und im föderativen Kompetenzgeflecht dezentralen (Steuerung der) Investitionskostenfinanzierung durch die Landesverwaltung (dualistische Finanzierung) andererseits. Hinzu kommen v.a. Fragen der Eröffnung selektiver Vertragsschlusskompetenzen auch im Krankenhausbereich sowie Ansätze zur Überwindung der Schnittstellen, insbesondere zwischen dem ambulanten und stationären Sektor.

Während die Integration von ambulantem und stationärem Bereich sowie das Vordringen des Krankenhauses in den ambulanten Bereich bereits im Zuge des GKV-WSG erleichtert wurden, befindet sich die Krankenhausfinanzierung - wie auch der Krankenhausmarkt überhaupt ${ }^{27}$ - weiterhin in einer höchst komplexen Phase des Umbruchs. Es galt zunächst, den Zeitraum bis zum Ende der sog. Konvergenzphase zur Einführung der DRG zu bewältigen, innerhalb dessen die Krankenhausbudgets bis zum 1. Januar 2009 an landesweit einheitliche Preise angeglichen wurden, sich den Landesbasisfallwerten anzunähern hatten. ${ }^{28}$ Der Gesetzgeber hatte sich bis dahin „auf gesetzliche Regelungen bis zum Ende der Konvergenzphase beschränkt“29 und das Vorhaben eines „Gesetz[es] zum ordnungspolitischen Rahmen der Krankenhausfinanzierung ab dem Jahr 2009“ (KHRG) zukünftiger Gesetzgebung überantwortet, die am 17. März ihren Abschluss fand. ${ }^{30}$ Im Hinblick darauf, da 3 weder der monistischen Finanzierung 31 noch selektivem Preiswettbewerb zum Durchbruch verholfen wurde, erfuhr bereits der Entwurf deutliche Kritik. 32

In der Tat fällt auf, daß sich das KHRG eher als Konglomerat an Einzelregelungen präsentiert, anstatt neue ,ordnungspolitisch“-konzeptionelle Grundprinzipien zu etablieren. Man mag dies als Konsequenz der bisherigen Anlage des DRG-Systems empfinden. Indes wäre eine stärker wettbewerbsorientierte Weiterentwicklung im vorgesehenen DRG-System möglich gewesen, ungeachtet der Frage seiner Weiterentwicklung. Während der 32seitige Entwurf des GKV-OrgWG in nahezu derselben Zahl Bezüge

27 Vgl. dazu Klauber/Robra/Schellschmidt (Hrsg.), Krankenhaus-Report 2006 (2007).

28 S. näher Leclerque/Friedrich, in: Klauber/Robra/Schellschmidt (Hrsg.), Krankenhaus-Report 2008/2009 (2009), S. 229 ff.; s. auch Braun//Rau/Tuschen, in: Klauber/Robra/Schellschmidt (Hrsg.), Krankenhaus-Report 2007 (2008), S. 3 (10): „Ziel der Konvergenzphase ist die Verbesserung der Ressourcenallokation durch Überführung der historisch krankenhausindividuell und kostenorientiert vereinbarten Krankenhausbudgets an ein leistungsbezogenes Preisniveau auf Landesebene im Jahr 2009“.

29 Vgl. BT-Drs. 16/10807, S. 1.

30 BGBl. I, S. 543.

31 S. aber unten I.3.4.2.2.

32 Vgl. etwa die auf der homepage des Bundestages abrufbaren Stellungnahmen der Einzelsachverständigen Neubauer, Drs. 16(14)90447(6), Ebsen, Drs. 16(14)90447(39), und Wasem, Drs. 16(14)90447(21). 
zum „Wettbewerb“ aufweist, ist davon im 48seitigen KHRG-Entwurf, von einer Bezugnahme auf das GKV-Wettbewerbsstärkungsgesetz einmal abgesehen, nur einmal die Rede. Dennoch bringt diese Passage ${ }^{33}$ die Überzeugung des Gesetzgebers von einem „leistungsorientierten Vergütungssystem“ zum Ausdruck, „,bei dem Wettbewerb zwischen den Krankenhäusern herrscht und Strukturveränderungen grundsätzlich erwünscht sind“".

Seit Beginn des Jahres 2009 steht auch die Entwicklung des Krankenhausmarktes im Kontext der Reform der allgemeinen Finanzierungsstrukturen der GKV. Von Bedeutung ist dies, weil sich Profil und Marktmacht der Kassen als Kostenträger und Vertragspartner verändern, insbesondere ein Fusions- bzw. Konzentrationsprozess eingesetzt hat, der in Verbindung mit den wettbewerbserheblichen Finanzierungsregelungen und auch mit der Einführung der insolvenzrechtlichen Vorschriften im Zusammenhang steht.

Vor diesem Hintergrund ist das Konzept der Steuerung durch Wettbewerb näher zu betrachten, v.a. die Justierung im Spannungsfeld zur Regulierung. Daß sich beide Ansätze jedenfalls aus sozialrechtlicher Warte nicht antinomisch gegenüber stehen müssen, zeigt bereits das Konzept vom „Wettbewerb um Qualität und Wirtschaftlichkeit“: „Wirtschaftlichkeit“ bedeutet hier keinen betriebswirtschaftlich autonom zu bestimmenden Ist-Zustand, sondern eine normative Größe, ein sozialrechtliches Gebot (§ 12 SGB V), dessen Einhaltung (vor allem) nach sozialrechtlich vorbestimmten und auszufüllenden (medizinischen) Standards zu beurteilen ist. Dasselbe gilt für die Qualität.

Um Potential und Grenzen der Mechanismen normativer Steuerung 34 im Bereich der Krankenhausversorgung entsprechend der zentralen Fragestellung der Untersuchung ergründen zu können, bedarf es zunächst eines allgemeinen Überblicks über die Systeme der GKV und der PKV. Das Krankenhausrecht wird dabei zunächst weitgehend ausgeblendet; es wird in den jeweiligen Funktionszusammenhängen ausführlich erörtert.

\section{Der institutionelle Rahmen}

\subsection{Gesetzliche und Private Krankenversicherung}

In der Bundesrepublik mit 82,2 Mio. Einwohnern ${ }^{35}$ erfolgt die versicherungsbezogene Vorsorge gegen das Risiko „Krankheit“ in erster Linie durch die Systeme von GKV und PKV. In beamtenrechtlichen Dienstverhältnissen wird ein Teil der Absicherung in Form der auf Beamtenrecht basierenden Beihilfe gewährt. Die GKV ist öffentlich-rechtlich organisiert. Träger sind die Krankenkassen als - nicht grundrechtsfähi-

33 BT-Drs. 16/10807. S. 30.

34 S. unten I.2.3.1.

35 Statistisches Bundesamt (StBA), Bevölkerungstand, 2007, abrufbar unter www.destatis.de. 
ge $^{36}$ - Körperschaften des öffentlichen Rechts mit Selbstverwaltung, die nach einzelnen Kassenarten gegliedert sind. ${ }^{37}$ Träger der PKV sind private Versicherungsunternehmen.

Das für die Untersuchung zentrale System der GKV gründet auf den Prinzipien „Solidarität" und „Eigenverantwortung“. 38 Der soziale Ausgleich kommt v.a. in der Familienversicherung zum Ausdruck. Ehegatten, Lebenspartner und Kinder von Mitgliedern genießen Versicherungsschutz und haben eigene Leistungsansprüche aus selbständigen Versicherungsverhältnissen. ${ }^{39}$ Die Leistungen in der GKV werden den Versicherten von den Krankenkassen zur Verfügung gestellt, sofern sie nicht der Eigenverantwortung unterliegen. ${ }^{40}$ Der Leistungsumfang ist für alle gesetzlich Krankenversicherten im Wesentlichen gleich. Es gibt nur wenige Unterschiede, ${ }^{41}$ z.B. unterschiedliche Präventionsangebote und damit verbundene Bonusleistungen ${ }^{42}$ sowie besondere (Integrationsund Koordinations-)Leistungen und damit verbundene Tarife im Rahmen besonderer Versorgungsformen. 43

Leistungen werden zur Verhütung und zur Früherkennung sowie zur Behandlung von Krankheiten erbracht. ${ }^{44}$ Sie müssen ausreichend, zweckmäßig und wirtschaftlich sein. ${ }^{45}$ Das in der GKV vorherrschende Prinzip ist das Sachleistungsprinzip, d.h., die Leistungen werden grundsätzlich als Sach- oder Dienstleistung erbracht. ${ }^{46}$ Es besteht jedoch auch die Möglichkeit, dem Patienten die von diesem verauslagten Kosten zu erstatten (Erstattungsprinzip). ${ }^{47}$ Die Leistungen werden von den zur Versorgung der Versicherten berechtigten Leistungserbringern wie Ärzten, Krankenhäusern und Apotheken erbracht. Ihre Beziehungen zu den Krankenkassen sind im Leistungserbringungsrecht besonders geregelt. ${ }^{4}$ Auch entfernter beteilige Akteure - wie zum Beispiel pharmazeutische Unternehmen - sind dort genannt. Im Jahre 2008 bestand Versiche-

36 S. Beschluss der 3. Kammer des Zweiten Senats des BVerfG vom 9. Juni 2004 - 2 BvR 1248/03, 2 BvR 1249/03 -, NZS 2005, S. 139, ferner BVerfGE 113, 165 (227), sowie bereits BVerfGE 39, $302(316)$.

$37 \S 4$ SGB V; $\S 21$ Abs. 1 SGB I, $\S \S 1$ und 2 SGB V.

$38 \S 1$ SGB V.

$39 \S 10 \mathrm{SGB} \mathrm{V}$.

$40 \S 2$ Abs. 1 S. 1 SGB V. Die Krankenkassen erbringen die Leistungen allerdings nicht selbst und nur in Ausnahmefällen durch Eigeneinrichtungen, vgl. § 140 SGB V.

41 Mühlhausen, Mitgliederwettbewerb innerhalb der gesetzlichen Krankenversicherung (2002), S. 185 ff.

$42 \S \S 20,25,26,65 \mathrm{a}$ SGB $\mathrm{V}$.

43 Vgl. § 53 Abs. 3 SGB V i.V.m. den dort genannten Vorschriften.

44 Vgl. § 11 SGB V.

45 Vgl. $\S 2$ Abs. 1, §§ 12, 70 Abs. 1 SGB V.

$46 \S 2$ Abs. 2 SGB V.

$47 \S 13$ SGB V.

$48 \S \S 69 \mathrm{ff}$. SGB V. 
rungsschutz der GKV für 70,2 Mio. Versicherte (Mitglieder und mitversicherte Angehörige). 49

Anders als in der GKV entscheiden im Recht der PKV ${ }^{50}$ private Verträge über Art und Höhe der Versicherungsleistungen. Hier gilt das Versicherungsvertragsgesetz, und die Inhalte der Versicherungsverträge richten sich nach den allgemeinen Versicherungsbedingungen für verschiedene Leistungen. Familienangehörige müssen mit eigenen Beiträgen, wenn auch meist günstigeren, eigens versichert und dafür eine Prämie gezahlt werden. Die Prämien in der PKV sind grundsätzlich ${ }^{51}$ alters-, geschlechts- und risikoabhängig. Zudem werden Rückstellungen für das Alter der Versicherten gebildet. In der PKV gilt das Kostenerstattungsprinzip. Ende 2007 verfügten 8,54 Mio. Personen über eine private Krankheitsvollversicherung. ${ }^{52} \mathrm{Gem}$. § 257 Abs. 2 SGB V erhalten v.a. die nur wegen Überschreitens der Jahresentgeltgrenze versicherungsfreien oder von der Versicherungspflicht befreiten und bei einem PKV-Unternehmen versicherten für sich und ihre Angehörigen (die nach $\S 10$ SGB V familienversichert wären) einen Beitragszuschuss von ihrem Arbeitgebeber.

Im Zeichen der Reform durch das GKV-WSG wurden die PKV-Unternehmen verpflichtet, ab dem 1.1.2009 eine „Basisversicherung“ mit spezifisch solidarischer Ausprägung anzubieten, speziell vor dem Hintergrund, daß eine Gruppe von Personen bisher ohne Versicherungsschutz geblieben war. Es sind bzw. waren dies z.B. Kleinunternehmer und finanziell in Bedrängnis geratene Selbständige, die von der Versicherungspflicht in der GKV befreit waren, die aber die Beiträge nicht mehr bezahlen konnten, oder solche, die aus dem Ausland zurückkehren und nicht versichert sind. ${ }^{53}$ Vor Einführung des Basistarifs bestand ein Kontrahierungszwang zur Versicherung dieser Personen seit dem 1.7.2007 im Standardtarif. Das Aufnahmerecht in den Basistarif ab 2009 bei jedem Versicherungsunternehmen steht bzw. stand allen in Deutschland nicht pflicht- oder freiwillig Versicherten, allen Beihilfeberechtigten, allen freiwillig GKVVersicherten mit sechs Monaten Wechselfrist sowie allen bisher PKV-Versicherten mit 6-Monatsfrist nach Einführung des Basistarifs zu. ${ }^{54}$ Der Beitrag darf den „Höchstbeitrag" der GKV nicht übersteigen; zudem wurde eine Sozialgrenze eingeführt, nach der sich der Beitrag für den Basistarif um die Hälfte vermindert, wenn allein durch seine

49 BMG, Gesetzliche Krankenversicherung, Kennzahlen und Faustformeln, 1.-4. Q. 2008, Stand. 4.3.2009, Ziff. 10a; abrufbar unter www.bmg.bund.de.

50 Neben den allgemeinen zivilrechtlichen Regelungen sind insbesondere die $\S \S 12-12 \mathrm{~g}$ und 81d des Versicherungsaufsichtsgesetzes (VAG) sowie $\S \S 193-208$ des Versicherungsvertragsgesetzes (VVG) maßgeblich.

51 Vgl. zum Basistarif sogleich.

52 Zahlenbericht der PKV 2007/2008, S. 27; Ende 2005 waren es demgegenüber nur 8,37 Mio. Personen, Zahlenbericht der PKV 2005/2006, S. 25.

53 Ausweislich der Gesetzesbegründung zum GKV-WSG handelt es sich um insgesamt 188.000 Personen (2003) ohne Krankenversicherungsschutz; BT-Drucks. 16/3100, Begründung, S. 94.

$54 \S 12$ Abs. 1b VAG i.d.F. des GKV-WSG mit Wirkung zum 1. Januar 2009 (Art. 46 Abs. 10). 
Zahlung Hilfebedürftigkeit i.S.d. SGB II bzw. SGB XII entstehen würde. 55 Dadurch entstehende Mehraufwendungen sind in einem Risikoausgleich auf alle beteiligten Versicherungsunternehmen im Sinne gleichmäßiger Belastungen zu verteilen (§ 12g Abs. 1 S. 2 HS. 2 VAG), während Mehraufwendungen, die im Basistarif auf Grund von Vorerkrankungen entstehen, auf alle im Basistarif Versicherten gleichmäßig verteilt werden müssen (§ 12g Abs. 1 S. 2 HS.1 VAG, § 203 Abs. 1 S. 3 VVG). In diesem „modifizierten $^{56}$ Standardtarif" waren im Jahr 2007 insgesamt 2.898 Personen versichert; bei 1.801 dieser Personen erfolgt eine Kappung des Beitrages auf den Höchstbeitrag der GKV. 57 Unter dem Gesichtspunkt der „Konvergenz der Systeme“ 58 auffällig ist die Erweiterung des Sicherstellungsauftrags der Kassenärztlichen (Bundes-)Vereinigungen auch auf die (zahn-)ärztliche Versorgung der im Standard bzw. Basistarif Privatversicherten gem. $\S 75$ Abs. 3a SGB V, wobei ausschließlich als Privatärzte tätige Ärzte nicht erfasst sind. 59

\subsection{Finanzierungsstrukturen und solidarischer Ausgleich in der GKV bis 2008}

Die Finanzierung der gesetzlichen Krankenversicherung beruht in erster Linie auf Beiträgen, 60 die zur Hälfte von den versicherungspflichtigen Beschäftigten (Arbeitern, Angestellten und zu ihrer Berufsausbildung Beschäftigten) ${ }^{61}$ und zur Hälfte von deren Arbeitgebern getragen werden. ${ }^{62}$ Bei versicherungspflichtigen ${ }^{63}$ Rentnern beteiligt sich zur Hälfte die Rentenversicherung, ${ }^{64}$ und freiwillig Versicherte, das sind Angehörige bestimmter leistungsfähiger Personengruppen, tragen die Beiträge allein, ${ }^{65}$ erhalten jedoch nach Maßgabe des $\S 257$ SGB V einen Zuschuss (vom Arbeitgeber). Freiwillig versichern 66 kann sich z.B. derjenige (Arbeiter oder Angestellte), dessen regelmäßiges Jahreseinkommen die so genannte Jahresarbeitsentgeltgrenze übersteigt. ${ }^{67}$ Wer Arbeits-

$55 \S 12$ Abs. 1c VAG i.d.F. des GKV-WSG mit Wirkung zum 1. Januar 2009 (Art. 46 Abs. 10).

56 Im bisher bracheneinheitlichen Standardtarif waren im Jahr 2007 insgesamt 31.046 Personen versichert, s. den Zahlenbericht der PKV 2007/2008, S. 30.

57 A.a.O.

58 Axer, Einbeziehung der PKV in die GKV, MedR 2008, S. 482 (492).

59 Axer, a.a.O., S. 488.

60 Vgl. $§ 220$ SGB V.

$61 \S 5$ Abs. 1 Nr. 1 SGB V. Der Kreis der Versicherten ist im übrigen wesentlich weiter gefasst: Auch Landwirte (Nr. 3), Künstler und Publizisten (Nr. 4), Studierende (Nr. 9) sowie weitere in $\S 5$ genannte Personengruppen sind erfaßt.

$62 \S 249$ Abs. SGB V.

63 Vgl. § 5 Abs. 1 Nr. 11 SGB V, zu Problemen der Vorversicherungszeit Just, in: Becker/Kingreen, SGB V, 2008, § 5 Rn. 48 ff.

$64 \S 249 \mathrm{a}$ SGB V.

$65 \S 250$ Abs. 2 SGB V.

$66 \S 9$ SGB V.

$67 \S 6$ Abs. 6 SGB V i.V.m. $\S 160$ SGB VI und $\S 4$ Abs. 1 der SozialversicherungsRechengrößenverordnung. Sie beträgt im Jahre $2009 € 48.600$ pro Jahr. Eine Sonderregelung gilt 
losengeld II nach dem SGB II bezieht, ist versicherungspflichtig;68 die Beiträge werden allerdings vom Bund (allein) getragen. 69

Die Höhe der Beiträge richtet sich nach der individuellen Leistungsfähigkeit des Versicherten, nach seinen beitragspflichtigen Einnahmen. ${ }^{70}$ Krankheits-, Risiko- oder Alterszuschläge werden grundsätzlich nicht erhoben. Der durchschnittliche allgemeine Beitragssatz, den die einzelnen Krankenkassen bis Ende des Jahres 2008 in ihren Satzungen individuell festlegten, ${ }^{71}$ betrug im Jahre 2008 (1.-4. Q) 14,0\% des beitragspflichtigen Einkommens. ${ }^{72}$ Hinzu kommt seit dem Sommer 2005 ein Zusatzbeitrag in Höhe von $0,9 \%$, den allein die Mitglieder zahlen. ${ }^{73}$ Die bis Ende 2008 kassenindividuell erhobenen Beiträge fielen demgemäß unterschiedlich aus, mussten sich indes streng am Finanzbedarf orientieren. V.a. über diesen „Beitragswettbewerb“74 fand ein Wettbewerb um Mitglieder statt. ${ }^{75}$ Denn die Mitglieder, ${ }^{76}$ also versicherungspflichtig Beschäftigte und freiwillige Mitglieder, haben seit 1996 das Recht, zwischen den Krankenkassen zu wählen, und - unter Beachtung gewisser Bindungsfristen - zu wechseln. ${ }^{77}$ Der Beitragswettbewerb galt vormals als der wichtigste Pfeiler im Mitgliederwettbewerb. Er war indes kein vollkommener, kein echter Preiswettbewerb, ${ }^{78}$ da die Krankenkassen an Grundprinzipien und Ausrichtung am Finanzbedarf gebunden sind. Gab es 1991 noch 1.209 Krankenkassen, ${ }^{79}$ waren es zum 1. Januar 2009 nur noch 202.80

Das neben der Mitversicherung Angerhöriger wesentliche solidaritätsorientierte Element, das Wettbewerb im Rahmen einer Solidargemeinschaft erst ermöglicht, ist der kassenartübergreifende Risikostrukturausgleich (RSA), der durch die Versichertenstruk-

für Versicherte, die am 31.12.2002 bereits versicherungsfrei und bei einem PKV-Unternehmen krankenversichert waren, vgl. $§ 6$ Abs. 7 SGB V (2009: € 44.100, s. § 4 Abs. 2 der vorgenannten Verordnung).

$68 \S 5$ Abs. 1 Nr. 2 a SGB V.

$69 \S 251$ Abs. 4 SGB V.

$70 \S 223$ Abs. 2 S. 1 ; $\S \S 226$ ff. SGB V.

$71 \S 194$ Abs. 1 Nr. 4 SGB V a.F.

72 BMG, Gesetzliche Krankenversicherung, Kennzahlen und Faustformeln 2008 (Stand: 4.3.2009), Ziff. 11.

$73 \S \S 241 \mathrm{a}, 249$ Abs. 1 SGB V.

74 Mühlhausen, Der Mitgliederwettbewerb innerhalb der gesetzlichen Krankenversicherung (2002), S. $167 \mathrm{ff}$.

75 Vgl. Becker, Gesetzliche Krankenversicherung zwischen Markt und Regulierung, JZ 1997, S. 534 (537).

76 Zur Mitgliedschaft $\S \S 186$ ff. SGB V.

$77 \S 173-175$ SGB V.

78 Mühlhausen Der Mitgliederwettbewerb innerhalb der gesetzlichen Krankenversicherung (2002), S. 176.

79 BMG, Pressemitteilung vom 24. Juni 2006, eingestellt am 25.5.2006, abrufbar unter www.bmg.bund.de (Presse, Archiv, Presse 2/2006, 25.6.2006: „Ulla Schmidt begrüßt erste länderübergreifende AOK-Fusion").

80 S. vor I.1. 
tur bedingte Einnahmeunterschiede und spezifisch risikobedingte Belastungsunterschiede der gesetzlichen Krankenkassen ausgleicht. ${ }^{81}$

\subsection{Das GKV-Wettbewerbsstärkungsgesetz}

\subsubsection{Organisationsstrukturen und Wettbewerbsgrundlagen}

Das GKV-WSG, dessen zentrale Regelungen v.a. mit Wirkung zum 1. April 2007, 1. Juli 2008 und 1. Januar 2009 in Kraft traten, ${ }^{82}$ verfolgt eine institutionell weit reichende Reform der GKV. Die Änderungen können hier nur kurz skizziert werden; Details normativer Steuerung bleiben der Betrachtung der rechtlichen Grundlagen bzw. der Wettbewerbsbeziehungen vorbehalten. Dies gilt auch für das Krankenhaus als Akteur, ${ }^{83}$ welches vom GKV-WSG z.B. im Hinblick auf die (Berechtigung zur) ambulante(n) Operation nach $\S 116 \mathrm{~b}$ SGB V sowie Qualitätssicherungsmaßnahmen erfasst wird.

Mit dem GKV-WSG kam es zu strukturellen Veränderungen im Bereich der Organisation der Krankenkassen, um nach der Vorstellung des Gesetzgebers erhöhten Wettbewerbs- und Leistungsanforderungen in wirtschaftlicher Weise gerecht zu werden. Es gelte „ungenutzte Potentiale für Kassenzusammenschlüsse“ zu befördern, und zwar auch über die Grenzen der Kassenarten hinweg. ${ }^{84}$ Dies wird ermöglicht durch Beschluß der Verwaltungsräte der beteiligten Krankenkassen; Voraussetzung dafür ist allerdings die Genehmigung der zuständigen Aufsichtsbehörden. Einer Straffung der Strukturen und dem Abbau von Bürokratie dient überdies die Schaffung des einheitlichen „Spitzenverband[es] Bund der Krankenkassen", 85 der die bestehenden (vormals ${ }^{86}$ sieben) Spitzenverbände ablöst. Er ist Körperschaft des öffentlichen Rechts und der Aufsicht durch das BMG unterstellt. Ihm obliegen u.a. der Abschluß von Verträgen und die Mitwirkung beim Erlaß von Richtlinien. 87

Besonderer Hervorhebung bedarf darüber hinaus die Einführung von Wahltarifen 88 , sei es für Selbstbehalte, besondere Versorgungsformen, für Tarife der Kostenerstattung oder die Übernahme der Kosten für Arzneimittel besonderer Therapieeinrichtungen (z.B. Homöopathie). Im übrigen wurde das Modell selektiver Verträge einzelner Kos-

81 Ausführlich unter II.5.1.1.

82 Vgl. Art. 46 Abs. 1, BGBl. I 2007, 378 (471). Im Übrigen sind - differenzierend - 11 weitere, unterschiedliche Zeitpunkte bestimmt.

83 Vgl. zum Überblick über die das Krankenhaus betreffenden Änderungen den Beitrag „GKV-WSG: Darstellung der krankenhausrelevanten Regelungen“ (ohne Autor), das Krankenhaus 2007, S. 199206.

$84 \S 171 \mathrm{a}$ SGB V.

$85 \S \S 217$ a ff. SGB V.

86 Zum 1.1.2009 haben sich alle Ersatzkassen zum vedk zusammengeschlossen, einschließlich der Gmünder Ersatzkasse, die zuletzt einziges Mitglied im AEV war, welcher zum 31.12.2008 aufgelöst wurde.

$87 \S 210$ Abs. 2 SGB V.

$88 \S 53$ i.d.F. des GKV-WSG. 
tenträger mit ausgesuchten Leistungserbringern gestärkt, z.B. im Rahmen der hausarztzentrierten wie der besonderen ambulanten ärztlichen Versorgung, mit Blick auf Verträge zur Integrierten Versorgung (IV), zur Versorgung mit Hilfsmitteln und Rabattverträge ${ }^{89}$ zur Arzneimittelversorgung. Speziell letztere lösen mannigfache Privilegierungen der rabattierten Arzneimittel aus, z.B. durch die Bevorzugung im Wege des aut idem oder die diesbezügliche Freistellung der Ärzte von Wirtschaftlichkeitsprüfungen. 90

\subsubsection{Insbesondere: Neuordnung der Finanzierungsgrundlagen}

Die größten Veränderungen sind mit der Neuordnung der Finanzierung entstanden. Die Krankenkassen legen den allgemeinen Beitragssatz nicht mehr autonom fest und erhalten insoweit keine individuellen Beiträge mehr. Stattdessen werden der allgemeine Beitragssatz i.H.v. zuletzt 14,9\% (Juli 2009) von der Bundesregierung festgelegt ( $\$ 241$ Abs. 1 SGB V) ${ }^{91}$ und die Beträge von den Krankenkassen und der DRV KBS ${ }^{92}$ als Einzugsstellen ${ }^{93}$ ebenso an den Gesundheitsfonds gezahlt (§ 271 Abs. 1 Nr. $1^{94}$ und Nr. $3^{95}$ SGB V), wie dort Beiträge der Direktzahler ( 252 Abs. 2 S. 1 SGB V) ${ }^{96}$ und der DRV Bund ( $§ 255$ Abs. 3 S. 4 SGB V) ${ }^{97}$ eingehen. Hinzu kommen Bundesmittel nach $\S 22198$ (vgl. § 271 Abs. 1 Nr. 5 SGB V). Der Grundsatz der Beitragsfinanzierung bleibt erhalten, § 220 Abs. 1 S. 1 SGB V.

89 Ausführlich Sichert, Neue Versorgungsformen und Rabatte für ausländische Leistungserbringer, EuR, Beiheft 2/2007, S. $101 \mathrm{ff}$.

90 Sichert, a.a.O., S. 123.

$91 \S 1$ der GKV-Beitragssatzverordnung (BGB1. I 2008, S. 2109, geändert durch Art. 14 des G zur Sicherung von Beschäftigung und Stabilität in Deutschland vom 5. März 2009, BGBl. I 2009, S. 416 (431), lautet: „Der paritätisch finanzierte Beitragssatz in der gesetzlichen Krankenversicherung beträgt, 14,0 Prozent. Der allgemeine Beitragssatz nach $\S 241$ des Fünften Buches Sozialgesetzbuch ist der um 0,9 Beitragssatzpunkte erhöhte Beitragssatz nach Satz 1.“

$92 \S 28 \mathrm{i} \mathrm{S.} 5 \mathrm{SGB}$ IV.

93 Einzuziehen, vgl. $\S \S 28 \mathrm{~d}, 28 \mathrm{~h}$ und 28i SGB IV, sind v.a. der Gesamtsozialversicherungsbeitrag (vgl. $\S 28 \mathrm{k}$ Abs. 1 S. 1 HS. 2, § 28e SGB IV, § 253 SGB V) sowie sonstige Krankenversicherungsbeiträge, z.B. der freiwillig Versicherten und Studenten (vgl. §§ 250, 254 SGB V), Beiträge aus Versorgungsbezügen ( $§ 256$ SGB V) oder der Rehabilitationsträger ( $\$ 251$ Abs. 1 und 2 i.V.m. $\S 252$ Abs. 2 SGB V).

94 I.V.m. § 28k Abs. 1 S. 1 SGB IV und $§ 252$ Abs. 2 S. 3 SGB V.

95 I.V.m. § 28k Abs. 2 SGB IV.

96 I.V.m. § 271 Abs. 1 Nr. 4 SGB V; vgl. § 251 Abs. 3, 4 und 4a: Künstlersozialkasse, Bundesämter für Zivildienst und Wehrverwaltung, zugelassene kommunale Träger bei Bezug von Arbeitslosengeld II, Bundesagentur für Arbeit. Für die Beiträge zur Pflegeversicherung agiert der Bund insoweit als Treuhänder, vgl. $\S 60$ Abs. 3 S. 1 HS. 2 SGB XI.

97 I.V.m. § 271 Abs. 1 Nr. 2 SGB V.

98 Satz 1 ebenfalls geändert durch Art. 13 des G zur Sicherung von Beschäftigung und Stabilität in Deutschland vom 5. März 2009, BGB1. I 2009, S. 416 (431): „Der Bund leistet zur pauschalen Abgeltung der Aufwendung der Krankenkassen für versicherungsfremde Leistungen 7,2 Milliarden Euro für das Jahr 2009 und 11,8 Milliarden Euro für das Jahr 2010 in monatlich zum ersten Bankarbeitstag zu überweisenden Teilbeträgen an den Gesundheitsfonds." Nach S. 2 erhöhen sich die Leis- 
Aus dem Gesundheitsfonds erhalten die Krankenkassen Zuweisungen zur Deckung ihrer Ausgaben ( $\$ 266$ Abs. 1 SGB V). Die Zuweisungen sind - bezogen auf die standardisierten Leistungsausgaben - nach den Grundsätzen des Risikostrukturausgleichs alters-, geschlechts- und risikoadjustiert. Der nunmehr allein ausgabenseitig angelegte RSA ist integraler Bestandteil des Gesundheitsfonds 99 und soll entsprechend der morbiditätsorientierten Weiterentwicklung dem Ziel fairer Wettbewerbsgrundlagen im Solidarsystem, insbesondere der Vermeidung von Risikoselektion, noch besser gerecht werden. Die Einzelheiten 100 zur Ermittlung der Zuweisungen und der Verfahrenssystematik regeln $\S 266$ ff. SGB V sowie die $\S \S 35 \mathrm{ff}$. RSAV. 101

Entgegen a.A. ist der Gesundheitsfonds (vgl. § 271 Abs. 1 SGB V) kein Sondervermögen des Bundes, sondern gemeinschaftliches Sondervermögen der Krankenkassen. ${ }^{102}$ Von der Vermögensträgerschaft indes ist die Verwaltungsträgerschaft zu unterscheiden: Verwaltungsträger ist der Bund und die für die Verwaltung zuständige Behörde, das Bundesversicherungsamt (BVA), als selbständige Bundesoberbehörde in ihrer Funktion als Durchführungsbehörde.

Soweit der Finanzbedarf einer Krankenkasse aus dem Fonds nicht gedeckt ist, hat sie von ihren Mitgliedern einen individuellen Zusatzbeitrag zu erheben. ${ }^{103}$ Soweit umgekehrt die Zuweisungen den Finanzbedarf übersteigen, kann die Kasse Prämien an ihre Mitglieder auszahlen, wenn hinreichend Rücklagen gebildet wurden. ${ }^{104}$ Zusatzbeitrag und Prämie sind nach Vorstellung des Gesetzgebers zusätzliche Wettbewerbsinstrumente. ${ }^{105}$ Sie sollen als kostenorientierter Wettbewerbsparameter, soweit sich dieser durchsetzt, den Mitgliederwettbewerb in ähnlicher Weise steuern wie vormals der Beitragssatzwettbewerb. 106 Der Zusatzbeitrag, den das Mitglied allein zu tragen hat, ist unmittelbar von diesem an die Kasse zu zahlen; er ist kein (vom Arbeitgeber zu zahlender) Gesamtsozialversicherungsbeitrag 107 , zumal kein Quellenabzug erfolgt. ${ }^{108}$ In Umkehrung dieser Grundsätze dürfen Prämien auch nur an Mitglieder ausgeschüttet werden und kommen den Arbeitgebern ungeachtet der bis auf 0,9\% paritätischen Finanzierung

tungen des Bundes in den Folgejahren um jährlich 1,5 Milliarden Euro bis zu einer jährlichen Gesamtsumme von 14 Milliarden Euro.

99 Pfohl/Sichert, Der Gesundheitsfonds: Sondervermögen des Bundes oder der Krankenkassen?, NZS 2009, S. 71 (72 f.).

100 Siehe Göpffarth, Wie der morbiditätsorientierte Finanzkraftausgleich funktioniert, Soziale Sicherheit 2009, S. 12 ff.

101 Näher unten II.5.1.2.1.

102 Ausf. Pfohl/Sichert, Der Gesundheitsfonds: Sondervermögen des Bundes oder der Krankenkassen?, NZS 2009, S. 71 (72 f.).

$103 \S 242$ Abs. 1 SGB V.

$104 \S 242$ Abs. 2 SGB V.

105 BT-Drs. 16/3100, S. 165.

106 S. unter II.5.1.2.2.

107 Vgl. § 28e SGB IV.

108 S. BT-Drs. 16/3100, S. 266; BT-Drs. 16/4247, S. 54, sowie $\S 251$ Abs. 6, § 252 Abs. 1 S. 2 und $\S 266$ Abs. 1 S. 1 SGB V. 
des (Großteils des) allgemeinen Beitragssatzes nicht zugute. ${ }^{109}$ Sowohl Zusatzbeitrag als auch Prämie können als Pauschale oder in Prozent der beitragspflichtigen Einnahmen in der Satzung festgelegt werden. ${ }^{110}$ Der Zusatzbeitrag ist auf ein Prozent der beitragspflichtigen Einnahmen des Mitglieds begrenzt (§ 242 Abs. 1 S 2 SGB V).

\subsection{Weiterentwicklung der Organisationsstrukturen:}

\section{Insolvenzfähigkeit auch der gesetzlichen Krankenkassen}

Im Zeichen der Weiterentwicklung des Wettbewerbs in der GKV erkannte der Gesetzgeber das wesentliche Bedürfnis, die „finanziell bedeutsame Ungleichbehandlung“ in Form der Geltung der Insolvenzordnung (InsO) nur für die bundesunmittelbaren Krankenkassen zu beseitigen. ${ }^{111}$ Gleichzeitig galt es seiner Auffassung nach zu bedenken, daß die bisherigen Regelungen über die Haftung bei Schließung einer Krankenkasse mit den wettbewerblichen Strukturen immer weniger in Einklang zu bringen waren, zumal auch Krankenkassen einer Kassenart zueinander im Wettbewerb stehen. ${ }^{112}$

Vor diesem Hintergrund bestimmt das Gesetz zur Weiterentwicklung der Organisationsstrukturen in der gesetzlichen Krankenversicherung (GKV-OrgWG) ${ }^{113}$ die Anwendung der Insolvenzordnung (s. § 171b Abs. 1 SGB V) vom 1. Januar 2010 an mit Ausnahme der nicht am Wettbewerb teilnehmenden landwirtschaftlichen Krankenkassen sowie der (unselbständigen Abteilung) der DRV, der Knappschaft Bahn See. ${ }^{114}$ Für die Kassen gelten damit, wie der als Rechtsgrundverweisung angelegte $\S 171 \mathrm{~b}$ Abs. 2 SGB $\mathrm{V}$ verdeutlicht, die Insolvenzgründe der Insolvenzordnung (vgl. §§ 17-19 InsO). Deren Anwendung bringt - wie noch zu zeigen sein wird ${ }^{115}$ - erhebliche Schwierigkeiten mit sich. Bereits zum 1. Januar 2009 ist die Haftung der Länder für Versorgungsansprüche und Ansprüche auf Insolvenzgeld entfallen. ${ }^{116}$ Des weiteren beinhaltet das GKVOrgWG v.a. Bestimmungen über das Verhältnis zum Recht der Schließung (aufgrund auf Dauer nicht mehr gesicherter Leistungsfähigkeit) ${ }^{117}$, über das Haftungssystem bei Schließung und Insolvenz ${ }^{118}$ sowie Normen betreffend die Vermeidung der Schließung oder Insolvenz von Krankenkassen. ${ }^{119}$ Unterschätzt und ihm Rahmen der Darstellungen zum GKV-OrgWG meist ausgeblendet wird die Bedeutung des Art. 6 des Gesetzes,

109 Vgl. auch Wolf, Der neue Zusatzbeitrag nach § 242 SGB V, GesR 2008, S. 567 (571).

110 Vgl. BT-Drs. 16/3100, S. 165, wenngleich explizit nur zur Erhebung des Zusatzbeitrages.

111 BT-Drs. 16/9599, S. 1; BT-Drs. 16/10609, S. 1 (Beschlussempfehlung und Bericht).

112 Jeweils a.a.O.

113 Vom 18. Dezember 2008, BGB1. I, S. 2426.

$114 \S 171 b$ Abs. 1 SGB V; zu den Ausnahmen BT-Drs. 16/9559, S. 25.

115 S. unter II.5.1.4.

$116 \S 171 \mathrm{c}$ SGB V.

117 Vgl. v.a. § 171 b Abs. 3 SGB V.

118 S. $\S 171 \mathrm{~d}$ SGB V.

$119 \S \S 172,265 a, 265 b$ SGB V. 
der wesentliche Regelungen der Risikostrukturausgleichsverordnung (RSAV) zur Ermittlung der Zuweisungen aus dem Gesundheitsfonds und zur Durchführung des Zahlungsverkehrs einschließlich Strukturanpassungen ${ }^{120}$ und Bescheidsystematik enthält.

\section{Kernfragen des Wettbewerbs und der Regulierung im Gesundheitswesen}

\subsection{Die Rolle der Krankenhausversorgung}

Im Jahre 2006 haben die Leistungsausgaben der GKV für die Krankenhausbehandlung erstmals die Grenze von $€ 50$ Mrd. überschritten. ${ }^{121}$ Mit 34,7\% Anteil an den Leistungsausgaben von $€ 151,12$ Mrd. (2008) ist dieser Bereich der mit Abstand kostenträchtigste. ${ }^{122}$ Diesem von jeher besonderen Anteil der Ausgabenlast entsprechend markiert der „Krankenhaussektor“ ein zentrales Feld gesetzlicher Reformbestrebungen zur Kostenkontrolle, -senkung und -begrenzung. Seit geraumer Zeit befindet sich dieser Sektor im Umbruch, der in maßgeblicher Abwendung von tradierter Steuerung durch Regulierung zunehmend wettbewerbsorientierten Gestaltungs-, Verdrängungs- und Kontrollmechanismen unterliegt. Zur Vergütung der stationären Leistungen ist ab dem Jahre 2003 für Akutkrankenhäuser ein leistungsorientiertes Fallpauschalensystem (DRG) eingeführt worden, dessen Weiterentwicklung hin auf ein landeseinheitliches Preisniveau zunächst bis Ende 2008 an- und entsprechend der Verlängerung der Konvergenzphase in modifizierter Form fortdauerte. ${ }^{123}$ Darüber hinaus ist zum Jahre 2000 - mit effektiven Anreizen ab dem Jahre 2004 - die IV zur Verzahnung vor allem des ambulanten und stationären Sektors ebenso ermöglicht und weiter forciert worden, wie seit 2004 das verstärkte Vordringen der Krankenhäuser in den ambulanten Bereich (z.B. die ambulante Erbringung spezialisierter Leistungen). Nicht zuletzt im Gefolge dieser Entwicklungen herrscht unter den Krankenhäusern ein starker Fusions- bzw. Verdrängungswettbewerb.

Der Ausbildung trans- und intersektoraler Versorgungskonzepte unter Einbeziehung der Krankenhäuser wird weiterhin besonderes Entwicklungspotential eingeräumt. Gerade in dieser Hinsicht war der Krankenhausbereich auch Gegenstand der Reform durch das GKV-WSG, ${ }^{124}$ während er im übrigen bis zum Abschluß der Entwicklung der DRG in Richtung auf ein landeseinheitliches Preissystem von der Reform weithin ausgeklammert und die Schaffung eines neuen Ordnungs- und Finanzierungsrahmens späteren Reformanstrengungen überlassen wurde, die in das KHRG mündeten.

120 Näher unten II.5.1.2.1.

121 BMG, Gesetzliche Krankenversicherung, Kennzahlen und Faustformeln; Stand: 4.3.2009.

122 BMG (www.bmg.bund.de), Kennzahlen der gesetzlichen Krankenversicherung, Ziff. 2.

123 Vgl. näher unten I.3.3.2., I.3.4.2.

124 Vgl. bereits den Gesetzesentwurf, BT-Drucks. 16/3100, S. 89. 
Eine zentrale Herausforderung für den Einsatz wettbewerbsorientierter normativer Steuerungseffekte im Gesundheitswesen besteht mithin darin, die vorherrschenden Strukturen weithin separierter Versorgungsbereiche zielgenau zu erfassen. Die Identifizierung und Bewertung aktuell und potentiell wirksamer Mechanismen setzen daher voraus, die Wirkungszusammenhänge sowohl in den einzelnen Versorgungssektoren des Gesundheitsweisen als auch für die besonderen Formen der Krankenhausbehandlung einzeln und sektorenübergreifend zu lokalisieren und zu bestimmen.

\subsubsection{Die Krankenhausbehandlung und ihre Formen}

Das deutsche Gesundheitswesen zeichnet sich traditionell und noch immer durch eine Abschottung der Versorgungsbereiche voneinander aus. Zur voll- oder teilstationären Krankenhausbehandlung des gesetzlich Krankenversicherten bedarf es grundsätzlich einer Einweisung durch einen ambulant tätigen niedergelassenen Arzt oder durch einen Notarzt in eines der nächst erreichbaren geeigneten Krankenhäuser, § 39 Abs. 2, 73 Abs. 4 SGB V. ${ }^{125}$ Von Notfällen abgesehen muß das Krankenhaus bei lediglich ambulant indizierter Behandlung den Patienten in aller Regel ab- bzw. an den niedergelassenen Arzt verweisen. ${ }^{126}$

Während die stationäre Behandlung im Krankenhaus im Akutstadium auch eine Frührehabilitation umfasst ( $\$ 39$ Abs. 1 S. 3 SGB V), verlaufen die anschließenden Phasen der Rehabilitation in den vom Krankenhaussektor getrennten Bereichen der ambulanten Behandlung oder einer stationären Versorgung in Rehabilitationseinrichtungen (vgl. $\S \S 40,107$ Abs. 2 SGB V), welche in gesondertem Verfahren einschließlich Begutachtung zu beantragen ist, § 40 SGB V. Im Zeichen eines ganzheitlichen Gesundungsprozesses kann sich die Abgrenzung der Sektoren ,(Frührehabilitation in) der stationären Krankenhausbehandlung“ und ,stationäre Behandlung mit Rehabilitationsleistungen" als schwierig und ggf. wenig effizient erweisen. 127 Der dem deutschen Gesundheitswesen insofern nach wie vor attestierte Mangel an patientenorientierter und kostengünstiger Abstimmung der Bereiche wird weiterhin als strukturelles Defizit erachtet.

Darüber hinaus existieren innerhalb des differenziert normierten Tätigkeitsbereichs der Krankenhäuser verschiedene, mitunter sektorengleiche ${ }^{128}$ Behandlungsformen. Die stationäre Behandlung im Krankenhaus ist lediglich eine, wenngleich als vollstationäre

125 Zur Frage des Wahlrechts des Versicherten unten II.1.1.1. und II.1.1.2.

126 Vgl. § 39 Abs. 1 S. 3 SGB V und die nachfolgend dargestellten Sonderformen ambulanter Behandlung. Siehe ferner Tuschen/Trefz, Krankenhausentgeltgesetz (2004), S. 14.

127 S. auch Hambüchen, Aktuelle Rechtsprechung des BSG zum Krankenhausrecht, GesR 2008, S. 393 (400).

128 S. Baumann (2007), in: juris-PK SGB V, § 140a Rn. 25, die von einem Sektor der „Versorgung mit Leistungen der Krankenhausbehandlung“" spricht; ferner Huster, in: Becker/Kingreen, SGB V (2008), § 140a Rn. 8, sowie ausf. unten II.3.1.4.3. 
Behandlung die Hauptform und zugleich Regelfall der Krankenhausbehandlung. ${ }^{129}$ Nach $\S 39$ Abs. 1 S. 1 SGB V wird die Krankenhausbehandlung im Leistungsbereich der gesetzlichen Krankenversicherung ,vollstationär, teilstationär ${ }^{130}$, vor- und nachstationär ( $§ 115 \mathrm{a}$ SGB V) sowie ambulant ( $(115 \mathrm{~b}$ SGB V) erbracht.“" Die vollstationäre Krankenhausbehandlung zeichnet sich durch die über Tag und Nacht (oder mindestens einen vollen Tagesablauf) ${ }^{131}$ hinweg ununterbrochene, physische und organisatorische Eingliederung des Patienten in das Versorgungssystem des Krankenhauses aus. ${ }^{132}$ Es besteht Vorrang der teil-, vor- und nachstationären sowie der ambulanten Behandlung vor der stationären Versorgung, § 39 Abs. 1 S. 2 SGB V. ${ }^{133}$ Die verschiedenen Formen der Krankenhausbehandlung folgen weithin eigenen Strukturbedingungen, Vergütungsregelungen, ${ }^{134}$ Investitionsbedürfnissen und Qualitätssicherungsstandards. ${ }^{135}$

Die gem. § 39 Abs. 1 S. 2 SGB V von den Krankenhausärzten zu treffende Entscheidung über die Erforderlichkeit der Krankenhausbehandlung ist auch in wirtschaftlicher Hinsicht von besonderer Bedeutung. Denn vereinbarte Entgelte sind unbeschadet besonderer vertraglicher Regelungen nach § 112 SGB V gem. § 109 Abs. 4 S. 3 SGB V i.V.m. der jeweiligen Pflegesatzvereinbarung nur zu zahlen, wenn die Versorgung im Krankenhaus i.S.d. § 39 Abs. 1 S. 2 SGB V erforderlich ist bzw. war. ${ }^{136}$ Entscheidend ist dabei, daß nach dem Beschl. des Großen Senates des BSG von 25.9.2007137 die vormals divergierenden Auffassungen des ersten und dritten Senates des BSG ${ }^{138}$ dahingehend abgelöst wurden, daß dem Krankenhausarzt fortan keine Einschätzungsprärogative mehr zukommt, sondern die Notwendigkeit gerichtlich uneingeschränkt zu überprüfen ist. Die exakte Beurteilung der Erforderlichkeit auf der Grundlage medizinischer Erfordernisse 139 - vom $B S G$ nachfolgend als ex ante-Prognose auf der Grundlage objektiver medizinischer Befunde und wissenschaftlicher Erkenntnisses zum Zeitpunkt der Behandlung bei verfügbarem Wissens- und Kenntnisstand überprüft ${ }^{140}$ - liegt damit im unmittelbaren ökonomischen Interesse des Krankenhauses. Dasselbe gilt für die Abgrenzung vollstationärer Krankenhausbehandlung und stationärer medizinischer Re-

129 Schmidt (2004/2006), in: Peters, Handbuch der Krankenversicherung, SGB V, § 39 Rn. 99, 130.

130 Z.B. in Dialyse-Stationen.

131 Zur Begrifflichkeit im Rahmen des Privatversicherungsrechts Schoenfeldt/Kalis, in: Bach/Moser, Private Krankenversicherung (2002), C Rn. 71.

132 Noftz (2008), in: Hauck/Noftz, SGB V, K § 39 Rn. 46 m.w.N.

133 Näher Becker, in: ders./Kingreen, SGB V (2008), § 39 Rn. 21 ff.

134 Näher unter I.2.1.5.

135 Siehe z.B. $\S \S 115$ b Abs. 1 S. 1 Nr. 3, S. 3 SGB V.

136 S. in vorliegendem Kontext Hambüchen, Aktuelle Rechtsprechung des BSG zum Krankenhausrecht, GesR 2008, S. 393 (401 f.).

137 SozR 4-2500, § 39 Nr. 10, S. 73 (83 Rn. 29).

138 S. die Nachweise im Beschl. des Großen Senates, a.a.O., S. 76 Rn. 11 ff.

139 A.a.O., S. 78 Rn. 15 ff.

140 BSG, Urt. v. 10.4.2008, SozR 4-2500, § 39 Nr. 12, Ls. 3; S. 86 ff. 
habilitation im Hinblick auf die Fortdauer der Notwendigkeit vollstationärer Krankenhausbehandlung, insbesondere bei chronisch psychiatrischen Fällen. ${ }^{141}$

In jüngerer Zeit erfolgte eine wettbewerbsorientierte Teilöffnung der Krankenhäuser für den ambulanten Bereich. Sie kommt über die Zulassung zum ambulanten Operieren nach $\S 115$ b SGB V hinaus ${ }^{142}$ gleichwohl nur in Betracht, wenn eine entsprechende Ermächtigung zur Teilnahme an der vertragsärztlichen Versorgung erteilt wurde (§ 116 SGB V), eine fachgebietsspezifische Ermächtigung bei Unterversorgung vorliegt ( $116 \mathrm{a}$ SGB V), ferner im Rahmen vertraglicher Vereinbarungen zur Durchführung strukturierter Behandlungsprogramme (§ 116b Abs. 1 i.V.m. § 137g SGB V), für Hochschul- (§ 117 SGB V) und Psychiatrische Institutsambulanzen ( 1118 SGB V) sowie ggf. im Rahmen integrierter Versorgung nach $\S \S 140 \mathrm{a}$ ff. SGB V. Die Zunahme der Möglichkeiten ambulanter Behandlung mit Blick auf $\S \S 115 \mathrm{~b}, 116 \mathrm{~b}$ und 140a ff. SGB $\mathrm{V}$ entspricht der sozialpolitisch motivierten Forderung ,ambulant vor stationär“.143 Auch die Erbringung bestimmter Katalogleistungen nach $\S 116 \mathrm{~b}$ Abs. 2-4 SGB V stellt eine Ausweitung in Richtung des ambulanten Bereichs dar, die jedoch weniger wettbewerblich als vielmehr planerisch dominiert wird.

Die Einteilung in voll-, teil-, vor- und nachstationäre sowie die ambulante Krankenhausbehandlung ist der Sache nach auch im Privatversicherungsrecht bekannt. Anders als durch das Sozialrecht wird im Privatversicherungsrecht allerdings nicht bestimmt, wie die Krankenhausbehandlung (als Krankenbehandlung), auf die ein Anspruch besteht, ${ }^{144}$ erbracht wird; vielmehr werden die Bedingungen für den durch die Krankheitskostenversicherung gewährten Ersatz für Aufwendungen normiert. ${ }^{145}$ Der stationären Behandlung sind entsprechende Tarifbedingungen zugeordnet. Ein Krankenhaustagegeld wird im Falle der Versicherung nur bei stationärer Behandlung gewährt, vgl. § 1 Abs. 1 lit. b MB/KK (2009). Abgrenzungsschwierigkeiten bestehen im Bereich der teilstationären Behandlung, die z.T. restriktiv und nicht als (voll-)stationäre Behandlung erfasst wird. ${ }^{146}$ Als ambulante Behandlungen gelten offenbar auch solche zur „Vorbereitung oder Nachbeobachtung". 147

Ihrer Bedeutung, Eingliederungstiefe und medizinischer Erforderlichkeit entsprechend machen die Kosten für die stationäre Krankenhausleistungen den überragenden

141 Hambüchen, Aktuelle Rechtsprechung des BSG zum Krankenhausrecht, GesR 2008, S. 393 (400).

142 Allein die Typologie der Behandlungsformen ist abschließend, vgl. Noftz (2008), in: Hauck/Noftz, SGB V, K § 39 Rn. 6.

143 S. etwa Tuschen/Trefz, Krankenhausentgeltgesetz (2004), S. 4.

$144 \S 27$ Abs. 1 Nr. 5 SGB V.

$145 \S 1$ Abs. 1a, § 4 Abs. 4 und 5 der MB/KK (2009); abgedruckt bei Rüffer/Halbach/Schimikowski, VVG, 2009, S. 1602 ff., ferner abrufbar unter www.pkv.de.

146 Schoenfeldt/Kalis, in: Bach/Moser, Private Krankenversicherung (2002), C Rn. 71.

147 Schoenfeldt/Kalis, a.a.O. Die ,vor- und nachstationäre Behandlung“ im Sinne des SGB V allerdings ist rechtsdogmatisch und angesichts vergütungsrechtlicher Konsequenzen eindeutig weder als ambulante noch als klassische stationäre Behandlung einzustufen, vgl. Rau (2005), in O/R/S/W/Z, SGB V, § 115a Rn. 12. 
Teil der auf den Krankenhaussektor entfallenden Leistungsausgaben aus. Dies trifft sowohl für private als auch für Kostenträger der GKV zu. In beiden Versicherungssektoren machten die Ausgaben im Jahre 2005 jeweils rund ein Drittel der Leistungsausgaben aus. ${ }^{148}$ Durch den Verband der PKV werden ,stationäre Leistungen“ grundsätzlich allgemein ausgewiesen; eine Differenzierung im Sinne der oben genannten Behandlungsformen erfolgt nicht. ${ }^{149}$ Bei der GKV entfielen lediglich 0,4\% der Leistungsausgaben für die Krankenhausbehandlung auf die vor- und nachstationäre Behandlung, auf das ambulante Operieren im Krankenhaus $0,8 \%$ und auf die teilstationäre Behandlung in Dialysestationen ca. $0,3 \%$.

Im Zuge konzeptioneller Weiterentwicklung der Krankenhausvergütung wird zunehmend eine Differenzierung innerhalb stationärer Leistungen postuliert, die notfallähnliche Leistungen von einem so genannten „elektiven Fallspektrum“ abgrenzt. ${ }^{150}$ Letzteres beinhaltet stationäre Leistungen, bei denen das Krankenhaus den Umständen nach ausgewählt werden kann bzw. die „planbar“ und insoweit potentiell dem Kassenwettbewerb zuzuordnen sind. Elektive Leistungen lassen sind ihrer Natur nach wettbewerblich organisieren, d.h. insbesondere selektivvertraglich vereinbarter Vergütung unterstellen; entsprechend müsste über die Pflicht zur Ausschreibung nachgedacht werden. ${ }^{151}$

\subsubsection{Zur Relation zwischen ambulanten und stationären Behandlungsformen 152}

Nimmt man den Betrachtungszeitraum seit der erstmaligen Erfassung ambulanter Operationen (2002) bis zum Jahr 2007 als groben Rahmen, ${ }^{153}$ so lässt sich folgendes feststellen:

Die Zahl vollstationärer Behandlungen hat sich seit 2002 wenig verändert. 17,17 Mio. Behandlungsfälle (20.883 je 100.000 Einwohner) im Jahr 2007 stehen 17,43 Mio (21.135 je 100.000 Einwohner) im Jahr 2002 gegenüber, wobei die Zahl zunächst bis auf 16,82 Mio. im Jahre 2006 abnahm und dann wieder anstieg. Stärker auffallend sind die Veränderungen im Bereich der vor-, nach- und teilstationären Behandlungen: 2002

148 Näher unter I.2.2.

149 Verband der privaten Krankenversicherung (Hrsg.), Die private Krankenversicherung, Zahlenbericht 2007/2008, S. 51, 53. Eine weitere Unterteilung erfolgt gleichwohl nach allgemeinen Krankenhausleistungen, Wahlleistung Chefarzt, Wahlleistung Unterkunft sowie ErsatzKrankenhaustagegeld.

150 Grundlegend Leber/Malzahn/Wolff, Elektiv wird selektiv, in: Klauber/Robra/Schellschmidt, Krankenhausreport 2007 (2008), S. $81 \mathrm{ff}$.

151 S. u. II.3.1.7.

152 Die nachfolgenden Angaben beruhen v.a. auf einer im November 2005 veröffentlichten Umfrage in der Zeit von April bis Juli 2005, an der sich 319 Krankenhäuser beteiligt haben: DKI (Hrsg.), Krankenhaus Barometer, Umfrage 2005, vgl. S. 1 und, zu Methodik und Stichprobenbildung, S. 67 f. Daten für das Jahr 2005 beinhaltet die Umfrage 2006, an der sich 341 Krankenhäuser beteiligten.

153 S. dazu StBA (Hrsg.), „Grunddaten der Krankenhäuser“ 2002 (2005) bzw. 2007 (2008), Ziff. 1.1.1. bzw. 2.12; soweit die Vergleichsdaten auch der Statistik $2007 \mathrm{zu}$ entnehmen sind, wurde darauf zurückgegriffen. 
wurden 1,17 Mio vor-, rd. 747.200 nach- und 376.473 teilstationäre Leistungen erbracht. Demgegenüber waren es 2007 gut 2,71 vor-, rd. 781.200 nach- und 675.082 teilstationäre Behandlungen. Der starke Anstieg der im Vorfeld einer vollstationären Behandlung, etwa als Voruntersuchungen, erbrachten Leistungen entspricht offenkundig einem Versorgungsmanagement, welches effizienter Belegungsplanung und kurzer Verweildauer geschuldet ist.

Im Jahre 2007 führten 1.309 der 2.087 Krankenhäuser insgesamt rd. 1,64 Mio. ambulante Operationen i.S.d. § 115 b SGB V durch. Im ersten Jahr der Aufzeichnung (2002) waren es 1.059 der 2.221 Häuser, die zusammen nur gut 575.000 Operationen durchführen. Während das Vordringen in den ambulanten Sektor der Zahl der Operationen nach deutlich zum Ausdruck kommt, steht diesem Zuwachs von über 1 Mio. Operationen ein eher moderater Anstieg des Anteils der Krankenhäuser gegenüber, in denen ambulant operiert wird, nämlich von $47,6 \%$ auf $62,7 \%$.

Detaillierte Analysewerte für einen Ausschnitt der Entwicklung liefert die Umfrage „Krankenhausbarometer“ des Jahres 2005: Danach gingen die auf den DRGFallpauschalenkatalog (2004) bezogenen vollstationären Leistungen der Krankenhäuser (ab 100 Betten) im Jahre 2004 mit 10.647 Fällen im Mittel um ca. 4\% gegenüber dem Vorjahr zurück. ${ }^{154}$ Gleichzeitig stieg die Zahl ambulanter Operationen nach $\S 115 \mathrm{~b}$ SGB V für das Jahr 2004 um 53\% gegenüber dem Vorjahr (638) auf durchschnittlich 977 ambulante Operationen. ${ }^{155}$ Krankenhäuser ab 600 Betten führten im Mittel 1.955 ambulante Operationen durch. Werden Erlöse aus ambulanten und stationären Leistungen als betriebliche Erlöse im engeren Sinne zusammengefaßt, beträgt der Anteil stationärer Leistungen 96,9\%, der Anteil ambulanter Leistungen 3,1\% (2004). ${ }^{156} 80 \%$ der Erlöse im ambulanten Bereich resultieren aus Krankenhaus- oder Chefarztambulanzen, $20 \%$ aus ambulanten Operationen nach $\S 115 \mathrm{~b}$ SGB V. Der Erlös je ambulanter Operation nach $\S 115$ b SGB V betrug durchschnittlich $€ 322,80^{157}$ Bezogen auf die Gesamtleistung eines Krankenhauses, würde diese durch die Anzahl der ambulanten Operationen gem. $\S 115 \mathrm{~b}$ SGB V und die Anzahl der stationären Fälle bestimmt, betrüge der Anteil der ambulanten Operationen im Mittel über alle Krankenhäuser 6,8\% im Jahre 2004 und 7,8\% im Jahre 2005. ${ }^{158}$ Der Anteil stieg in den Jahren 2006 (8,7\%) und 2007 $(9,3 \%)$ nochmals an. 159

154 Krankenhaus Barometer, Umfrage 2005, S. 4, 10 f. Eine Aussage zum Rückgang stationärer Fallzahlen insgesamt kann mit Blick auf die 2003 und 2004 gezahlten sonstigen Entgelte (s. § 6 Abs. 1 KHEntgG) und mögliche Veränderungen der Abrechnungsbestimmungen allerdings nicht getroffen werden, a.a.O., S. 11.

155 A.a.O., S. 10, 12. Gegenüber 2004 ist für das Jahr 2005 nochmals ein Anstieg von 16\% zu verzeichnen, vgl. Krankenhaus Barometer, Umfrage 2006, S. 35.

156 A.a.O., Umfrage 2005, S. 14.

157 A.a.O., S. 12. Im Jahre 2005 waren es $€$ 311, Krankenhaus Barometer, Umfrage 2006, S. 20.

158 Krankenhaus Barometer, Umfrage 2006, S. 19.

159 Krankenhaus Barometer, Umfrage 2008, S. 17. 


\subsubsection{Das Krankenhaus im Blickpunkt neuer Versorgungs- und}

Kooperationsformen

Neben der Teilöffnung der Krankenhäuser für den ambulanten Bereich erfasst der Wettbewerb in besonderer Weise zwei Bereiche, nämlich die sektorenübergreifende Versorgung und die Erweiterung der Kooperationsmöglichkeiten vor allem der Leistungserbringer. In diesem Sinne sind „Herzstücke“160 der Reformansätze des GMG161 die Flexibilisierung und der Ausbau der im Jahre 2000 eingeführten sektorenübergreifenden Integrierten Versorgung (IV) ${ }^{162}$ sowie die Zulassung Medizinischer Versorgungszentren (MVZ) ${ }^{163}$ als neue Organisationsform. Zusammen mit der ambulanten Versorgung im Rahmen strukturierter Behandlungsprogramme ${ }^{164}$ soll dadurch ein „Wettbewerb zwischen verschiedenen Versorgungsformen“ möglich werden, der Innovationen beschleunigt und die Möglichkeit eröffnet, Effizienzreserven zu erschließen. ${ }^{165}$ Der Wettbewerb der Versorgungsformen ist gleichfalls ein solcher der Kooperationsformen, zwischen MVZ als „Kooperation unterschiedlicher ärztlicher Fachgebiete untereinander sowie mit nichtärztlichen Leistungserbringern“166, der IV als „Kooperation zwischen den einzelnen Versorgungsbereichen" 167 sowie zwischen verschiedenen Gestaltungsformen der MVZ und der IV und zwischen Formen mit und ohne Beteiligung auch der Träger der MVZ (oder ihrer Gemeinschaften) an der IV, vgl. § 140b Abs. 1 Nr. 3 SGB V.

\subsubsection{Das Krankenhaus - herausragender Akteur unter den Leistungserbringern}

Krankenhäuser sind umfassend in neue Versorgungsformen eingebunden und spielen angesichts der herkömmlichen Strukturen und der Kostenintensität des „Krankenhaussektors“ eine herausragende Rolle. Sie verfügen im Bereich komplexer Versorgungsstrukturen, welche Planung, Kalkulation, Investition und Vertragsgestaltung erfordern, oft über die erforderlichen Managementkapazitäten, ${ }^{168}$ die andere Leistungserbringer (z.B. Ärzte) eher nicht mitbringen, unbeschadet der zuweilen mangelnden Vertrautheit mit den Strukturen der ambulanten Versorgung im Zuge der Gründung eines MVZ. Ihnen kommt damit häufig ein strukturelles Übergewicht bei Vertragsschlüssen und Kooperationen zu. Trotz angespannten Budgets verfügen sie eher über entsprechende In-

160 S. Zwingel/Preißler, Das Medizinische Versorgungszentrum (2004), Kap. 1 Rn. 7-9, S. 20.

161 GKV-Modernisierungsgesetz (GMG), v. 14.11.2003, BGBl. I, 2003, S. 2189; siehe BT-Drucks. $15 / 1525$, S. $1,71 \mathrm{ff}$.

$162 \S \S 140$ a bis d SGB V.

163 Vgl. § 95 Abs. 1 S. 2 und 3 SGB V.

$164 \S 116$ a und $\mathrm{b}$ SGB V.

165 BT-Drucks. 15/1525, S. 74.

166 BT-Drucks. 15/1525, S. 108.

167 BT-Drucks. 14/1245, S. 55.

168 Vgl. für private Krankenhausketten, Amelung, in: ders./Meyer-Lutterloh/Schmid/Seiler/Weatherly, Integrierte Versorgung und medizinische Versorgungszentren (2006), S. 38. 
vestitions- und Finanzierungsvolumina, auch, um weitere Leistungserbringer für Kooperationsgemeinschaften anzuwerben, zumal allein (noch) 2.087 Krankenhäuser (2007) ${ }^{169}$ den stationären Krankenhaussektor ausmachen.

\subsubsection{Integrierte Versorgung (IV)}

Eine die Leistungssektoren überschreitende bzw. inderdisziplinär-fachübergreifende Versorgung ermöglichen konzeptionelle Vereinbarungen zur ,integrierten Versorgung“. Entsprechende Verträge regeln die integrierte Versorgung der freiwillig 170 teilnehmenden Versicherten im Verbund mehrerer Leistungserbringer. ${ }^{171}$ Aus ihnen ergeben sich das krankenhausspezifische Versorgungsangebot und die Voraussetzungen seiner Inanspruchnahme, ${ }^{172}$ Bedingungen für die Teilnahme der Versicherten, ${ }^{173}$ die Vergütung, ${ }^{174}$ besondere Tarife, ${ }^{175}$ die Finanzierung ${ }^{176}$ und ggf. die Einrichtung von Leitstellen zur Leistungssteuerung sowie zur Gewährleistung. ${ }^{177}$ Es gibt indikationsbezogene Modelle ${ }^{178}$, solche für ganze Krankheitsbilder und solche für umfassendes CareManagement mit Budgetverantwortung. Organisationsspezifisch lassen sich verschiedenste „Gesellschafts-“, „Kooperations-“ und „Einkaufsmodelle“ unterscheiden. ${ }^{179}$ Klinische Pfade bestimmen das Case Management, wie bspw. für einen IV-Vertrag zur Frühversorgung von Demenzpatienten unter Beteiligung (der Gedächtnisambulanz) einer Psychiatrischen Klinik dokumentiert. 180

„Die für die ambulante Behandlung im Rahmen der IV notwendige Versorgung mit Arzneimitteln“, so lautet der mit dem GKV-WSG um S. 5 erweiterte § 140a Abs. 1 SGB V, ,soll durch Verträge nach $\S 130$ a Abs. 8 erfolgen“. Somit können Managementgesellschaften ( $§ 140$ b Abs. 1 Nr. 4 SGB V) seit dem AVWG nun an beiden Arten von Verträgen beteiligt sein; Leistungserbringer ebenso wie Pharmaunternehmen erwä-

169 Näher unter I.3.2.2.

$170 \S 140 \mathrm{a}$ Abs. 1 S. 2 SGB V.

171 Vgl. § 140a Abs. 1 S. 1, Abs. 2 S. 1 SGB V.

$172 \S 140$ a Abs. 1 S. 4 SGB V.

173 Vgl. etwa $\S 4$ der „Vereinbarung einer integrierten Versorgung gem. § 140 b SGB V“ zwischen der Praxisgemeinschaft Ambulantes Operationszentrum Kronshagen GbR, der Praxisklinik Kronshagen GmbH \& Co. KG, der Techniker Krankenkasse und der KV Schleswig-Holstein, SchleswigHolsteinisches Ärzteblatt 12/2003, S. 66 ff.

$174 \S 140 \mathrm{c}$ Abs. 1 SGB V.

175 Vgl. § 53 Abs. 3 SGB V.

176 Einschließlich der durch $\S 140$ d Abs. 4 SGB V vorgegebenen Preisdifferenzierung, Bohle, a.a.O., S. $31 \mathrm{f}$.

177 Bohle, a.a.O., S. 9 (29 f., 32 ff.).

178 Greuèl, Neue Steuerungsansätze im Gesundheitswesen durch Integrierte Versorgung, Betreuungsmanagement 2005, S. 10 (12 f.).

179 Bohle, in: ders., Vertragsgestaltung in der Integrierten Versorgung (2005), S. 9 (25).

180 Siehe Fellgiebel/Schanz/Huwer, IV-Vertrag zur Frühversorgung von Demenzpatienten mit der BARMER Ersatzkasse, das Krankenhaus 2007, S. 318 ff. 
gen, sich an diesen Gesellschaften zu beteiligen. ${ }^{181}$ Zudem „sollen“ diese Verträge nach $\S 140 \mathrm{a}$ Abs. 1 S. 5 SGB V n.F. ,eine bevölkerungsbezogene Flächendeckung der Versorgung ermöglichen“. Dies deutet auf eine neue bzw. besondere Form der Regelversorgung, jedenfalls auf eine Hinwendung zum Care-Management mit Budgetverantwortung. ${ }^{182}$

Wichtig für Krankenhausleistungen ist die Regelung, der zufolge Integrationsleistungen des Krankenhauses aus der Anschubfinanzierung nur insoweit zu bezahlen sind bzw. waren, als sie nicht zu den mit dem Budget aus DRG und BPflV zu vergütenden Grundleistungen gehör(t)en. ${ }^{183}$ Erfasst sind mithin nur zusätzliche Leistungen sowie der Koordinierungs- und Dokumentationsaufwand. ${ }^{184}$ Denkbar sind auch die Vergütung von Mehr-Mengen und die Erweiterung auf bisher nicht als Krankenhausleistung vereinbarte Leistungen. 185

Statt selbst mit der Krankenkasse zu kontrahieren, können Krankenhausträger mit Managementgesellschaften ${ }^{186}$ kooperieren (und konkurrieren), die Leistungen zur IV quasi „einkaufen“ und ihrerseits mit den Krankenkassen Versorgungsverträge schließen, $\S 140 \mathrm{~b}$ Abs. 1 Nr. 4 SGB V. Im Verbund der Leistungserbringer können sich zwischen Krankenhäusern und anderen Leistungserbringern i.ü. umfassende Beziehungen zur Binnenkooperation entwickeln.

Mit dem GKV-WSG wurde der Kreis der Vertragspartner auf Pflegekassen und zugelassene Pflegeeinrichtungen ausgedehnt (§ 140b Abs. 1 Nr. 5 SGB V i.V.m. § 92b SGB XI). Das KHRG gestattet zudem die Einbeziehung der Praxiskliniken nach $\S 115$ Abs. 2 S. 1 Nr. 1 SGB V, vgl. $§ 140 b$ Abs. 1 Nr. 7 SGB V. 187

\subsubsection{Medizinische Versorgungszentren}

Als Leistungserbringer dürfen Krankenhäuser gem. § 95 Abs. 1 S. 3 HS. 2 SGB V medizinische Versorgungszentren gründen. MVZ sind fachübergreifende ärztlich geleitete Einrichtungen, 188 in denen in das Arztregister eingetragene Ärzte als Angestellte

181 Grenzen ergeben sich in diesem Zusammenhang insbesondere aus dem ärztlichen Berufsrecht.

182 Das Integrations-Vertrags-Projekt „Gesundes Kinzigtal“ zwischen der gleichnamigen Managementgesellschaft und der AOK Baden-Württemberg geht in diese Richtung: Das Vertragsgebiet umfaßt 17 Städte und Gemeinden mit insgesamt 64.000 Menschen; 1550 Patienten zur Vollversorgung waren bis März 2007 eingeschrieben, maßgeblich für 13\% der Gesamtkosten; ausf. Hermann u.a., Das Modell „Gesundes Kinzigtal“, GuS 2006, S. 21 ff.

$183 \S 140 \mathrm{~d}$ Abs. 4 SGB V i.V.m. $\S$ 3, 4 KHEntgG und $\S 6$ BPflV. Eingehend zur Problematik Quaas, in: ders./Zuck, Medizinrecht (2008), S. 192 ff., ferner unten II.3.1.4.3, auch zur Finanzierung ab 2009.

184 Hencke (2007), in: Peters, Handbuch der Krankenversicherung, SGB V, § 140d Rn. 6.

185 Siehe Quaas, in: Wenzel (Hrsg.), Handbuch des Fachanwalts Medizinrecht (2007), Kap. 12, S. 1174.

186 Diese selbst sind keine Leistungserbringer.

187 BT-Drs. 16/11429, S. 42, 65.

188 Die Idee zu MVZ geht zurück auf das Modell der im frühen 20. Jh. gegründeten und nach Ende des zweiten Weltkrieges in der DDR existierenden Polikliniken. 
oder Vertragsärzte tätig werden. 189 Die Leistungen des an der vertragsärztlichen Versorgung teilnehmenden MVZ beziehen sich allerdings nur auf den ambulanten Bereich. ${ }^{190}$ Die „Bündelung der medizinischen Kompetenz unter einem Dach“, die „strukturierte Behandlung durch engere Zusammenarbeit der Ärzte“ und die „Gemeinschaftliche Nutzung der Medizintechnik" sind gleichwohl die mit der Reform verbundenen zentralen Vorteile und Chancen der Versorgungszentren. ${ }^{191}$ Die abgestimmte fachübergreifende Ausrichtung an den Bedürfnissen des Patienten liegt als ,potentieller Wettbewerbsvorteil“ 192 in der Struktur der MVZ begründet, 193 die oft als „Nukleus für eine Integrationsversorgung“194 und ,geborene(n)“195 IV-Vertragspartner (§140b Abs.1 Nr. 3 SGB V) ${ }^{196}$ bezeichnet werden.

\subsubsection{Das Vordringen der Krankenhäuser in den ambulanten Bereich als}

Ausdruck des Wettbewerbs um den Erwerb von Krankenhäusern

Mit dem Vordringen der Krankenhäuser in den ambulanten Bereich sind oftmals strategische Interessen zugunsten auch des stationären Bereichs verbunden, um in einem zunehmenden Verdrängungs- und Fusionswettbewerb privater Krankenhausträger zu bestehen. Dies wird deutlich in einer Entscheidung des Bundeskartellamts (BKartA) vom März 2005 betreffend den „Wettbewerb um den Erwerb von Krankenhäusern.“197 Dem BKartA zufolge ist die Beteiligung an einem MVZ von strategischer Bedeutung im Wettbewerb um den Zugang zu Absatzmärkten. Eine Beteilung erweitere die „Möglichkeit von Krankenhäusern, auf ambulante Einrichtungen und damit auf potentielle Pati-

$189 \S 95$ Abs. 1 S. 2 SGB V.

190 S. unter Verweis auf $\S 73$ SGB V Andreas, Medizinische Versorgungszentren, ArztRecht 2005, S. 144; ferner Zwingel/Preißler, Das Medizinische Versorgungszentrum (2004) Kap. 4 Rn. 15 m.w.N.

191 A.a.O.

192 Altendorfer/Merk/Jensch, Das Medizinische Versorgungszentrum (2004), S. 63.

193 Die Besonderheiten der MVZ müssen überdies bei der Zusammenfassung der im einheitlichen Bewertungsmaßstab aufgeführten Leistungen zu Leistungskomplexen oder Fallpauschalen besonders berücksichtigt werden, vgl. § 87 Abs. 2a SGB V. S. auch BT-Drucks. 15/1525, S. 105.

194 Richter-Reichhelm, Faire Wettbewerbschancen für alle, Forum für Gesundheitspolitik 2004, S. 165 (167).

195 Richter-Reichhelm, a.a.O., S. 168. Siehe kritisch dazu Ratzel, in: Dahm/Möller/Ratzel, Rechtshandbuch Medizinische Versorgungszentren (2005), Kap. XIV. Rn. 4.

196 Überdies können die Krankenkassen auch Verträge zur hausarztzentrierten Versorgung mit MVZ abschließen, vgl. § 73b Abs. 2 Nr. 2 SGB V.

197 Beschlussabteilung B 10 - 109/04, Öffentliche Version, Ziff. 30 f. (Ziff. 33). „Aufgrund der Fallpauschalenregelung (...) werden sich die Verluste unterausgelasteter oder ansonsten unwirtschaftlicher Krankenhäuser weiter erhöhen, so dass sich immer mehr öffentliche und freigemeinnützige Träger gezwungen sehen werden, ihre Krankenhäuser entweder zu schließen oder an private Krankenhausbetreiber zu veräußern. “ Das BKartA hat der Rhön-Klinikum AG letztlich untersagt, ,,sämtliche Geschäftsanteile der Städtisches Krankenhaus Eisenhüttenstadt GmbH von der Stadt Eisenhüttenstadt zu erwerben“ (a.a.O., Tenor, Ziff. 1). 
entenströme Einfuß zu gewinnen.“198 Für die Krankenhäuser „stellt die Ausweitung ihrer Tätigkeit in den ambulanten Bereich nicht nur ein zusätzliches Tätigkeitsfeld dar, das zu einer Ertragsstabilisierung und Umsatzausweitung führt“, sondern bewirkt „Synergieeffekte für ihr Kerngeschäft“ durch Nutzung der „Tätigkeit zur Anwerbung von Krankenhauspatienten bzw. zur Einweisungssteuerung." "199 Eine konsequente Verfolgung dieser Strategie wird zu einer attraktiven Angebotspalette führen, die Verhandlungsvorteile u.a. für den Bereich der IV zur Folge hat. Wegen der Ausweitung auf den ambulanten Bereich müssen daher auch Krankenhäuser ,,von den Niedergelassenen sehr aufmerksam beobachtet und als ernstzunehmende Konkurrenzen eingeschätzt werden.“200

Mit dem GKV-WSG soll die ambulante Behandlung im Krankenhaus weiter forciert werden. Das betrifft insbesondere die Erbringung hochspezialisierter Leistungen sowie die Behandlung seltener Erkrankungen sowie solcher mit besonderen Krankheitsverläufen, § 116b Abs. 2 SGB V, abgesehen von der Teilnahme an DMP (Abs. 1). Die den Krankenkassen ursprünglich zur Ergänzung der vertragsärztlichen Versorgung zugewiesene, jedoch kaum genutzte ${ }^{201}$ Kompetenz zum Vertragsschluß entfällt. Stattdessen fällt die Entscheidung nach Antrag und unter Nennung der betreffenden Leistungen und Erkrankungen im Rahmen der Krankenhausplanung unter Berücksichtigung der vertragsärztlichen Versorgungssituation.

\subsubsection{Wettbewerb und Vergütungsstrukturen}

Besonderer Ansatzpunkt normativer Steuerung im Krankenhauswesen sind die Vergütungsstrukturen. Aus wettbewerbsorientierter Perspektive stellen sich für den Krankenhaussektor zwei Grundfragen: erstens, ob das Vergütungssystem als solches leistungsorientiert und effizient ausgestaltet ist, und zweitens, ob - bzw. inwieweit - auch die Investitionskosten Teil der Vergütung werden, d.h. der Vergütungsmechanismus zum finanzwirtschaftlich weithin exklusiven Steuerungsmodell avanciert. Im übrigen überwiegt im Rahmen der Investitionskostenfinanzierung seitens der öffentlichen Hand der regulatorische Ansatz, auch wenn die Förderung einen wesentlichen Faktor bzw. eine maßgebliche Strukturkomponente für die Aufstellung der Krankenhäuser im Wettbewerb darstellt.

198 A.a.O., Ziff. 130, S. 54.

199 A.a.O., Ziff. 132, S. 55.

200 Richter-Reichhelm, Faire Wettbewerbschancen für alle, Forum für Gesundheitspolitik 2004, S. 165 (168).

201 S. BT-Drucks. 16/31000, S. 139. 


\subsubsection{Leistungsorientierung der Vergütung}

Unter Wettbewerbsgesichtspunkten steht die Vergütung der allgemeinen Krankenhausleistungen, weniger die ambulante 202 Behandlungsform, im Blickpunkt.

Wettbewerbsorientierte Vergütungsmechanismen basieren zum einen darauf, daß sie nicht den korporatistisch agierenden Verbänden, sondern den Einzelakteuren Vergütungsautonomie zuzuweisen, eigene Vergütungsvereinbarungen zu treffen; sie werden durch Verantwortung und Vertragsgestaltungskompetenz zu kostengünstigem Wirtschaften angehalten. Dabei sind die Krankenkassen in Anbetracht direkter Vergütungsbeziehungen um preiswerte Krankenhausversorgung, die Krankenhäuser um Vertragsabschlüsse sowie ggf. die Akquisition zusätzlicher Vergütungsanteile und demgemäß um Rationalität und Effizienz bemüht. Dies weist in Richtung (Selektiv-)Vertragswettbewerb, von dem im Rahmen der Beziehungen der Marktteilnehmer noch ausführlich die Rede ist. 203

Bedeutsamer im gegebenen System ist die leistungsorientierte Ausgestaltung der Vergütung der allgemeinen Krankenhausleistungen. Im Zeichen des Wettbewerbs geschieht dies durch „leistungsorientierte“ Fallpauschalen. Dahinter verbirgt sich heute ein ebenso gewichtiges wie komplexes und neuartiges System; weithin undifferenzierte Fallpauschalen sind im Recht der Vergütung bereits seit längerem bekannt, etwa durch die Bundespflegesatzverordnung von 1973 (BPflV), die tagesgleiche und vollpauschalierte Pflegesätze normierte. ${ }^{204}$ Auf weitere (z.B. fachabteilungsbezogene) ${ }^{205}$ Tagespauschalen (vgl. § 115a Abs. 3 SGB V) sowie sonstige Vergütungsformen im ambulanten Bereich (§ 115b Abs. 5; § 116b Abs. 5 SGB V) soll hier jedoch zunächst ebenso wenig eingegangen werden wie auf den Kanon sonstiger und Zusatzentgelte bzw. Pauschalierungen nach der Bundespflegesatzverordnung, soweit das DRG-System (noch) keine Anwendung findet. ${ }^{206}$

Auf der Grundlage des $\S 17 \mathrm{~b}$ des Krankenhausfinanzierungsgesetztes (KHG) und der im Jahre 2005 erstmals ausgehandelte Fallpauschalenvereinbarung (FPV 2005) wurde nach Vorgabe des Fallpauschalengesetzes (2002) 207 ein ,durchgängiges, leistungsorientiertes und pauschalierendes Vergütungssystem“ eingeführt und in ersten Schritten umgesetzt. „Die leistungsorientierte Vergütung der Krankenhäuser“, so die Gesetzesbe-

202 Insbesondere nicht die Vergütung ambulanter Behandlungen durch Krankenhäuser bei Unterversorgung nach $\S 116 \mathrm{a}$ SGB V, die nach den Grundsätzen der vertragsärztlichen Gesamtvergütung vorzunehmen sind, § 120 Abs. 1 SGB V, welche unbeschadet einer gewissen Privilegierung von Kooperationsformen im EBM wenig wettbewerbsorientiert ausfällt.

203 S. II.3.1.4.

204 Patt, System und Kontrolle der Preisbildung für Krankenhausleistungen (1996) S. 45 f.; Tuschen/Trefz, Krankenhausentgeltgesetz (2004), S. 19 ff.

205 Rau (2007), in: O/R/S/W/Z, SGB V, § 115a Rn. 27.

206 Siehe Tuschen/Trefz, Krankenhausentgeltgesetz (2004), S. 99 ff.

207 Gesetz zur Einführung des diagnose-orientierten Fallpauschalensystems für Krankenhäuser (Fallpauschalengesetz - FPG), BGBl. I, S. 1412. 
gründung, ${ }^{208}$,wird zu mehr Wettbewerb und zu einer stärker am tatsächlichen Bedarf orientierten Entwicklung der Leistungsstrukturen und Leistungskapazitäten führen“. Seitdem richtet sich die Vergütung (ergänzend) nach dem Krankenhausentgeltgesetz (KHEntgG). Bei dem aus Australien bekannten und zunächst grundsätzlich übernommenen ${ }^{209}$ System der „Diagnosis Related Groups“ (DRG, vgl. § 17b Abs. 2 KHG) wird jedem stationären Behandlungsfall eine der nunmehr 1.192210 Fallgruppen zugeordnet. ${ }^{211}$ Jede Gruppe wird in Relation bewertet, (seit 2005) einem Basisfallwert zugeordnet ( $§ 17 \mathrm{~b}$ Abs. 3 S. 5 KHG) und durch Multiplikation beider Parameter die Vergütungshöhe bestimmt. ${ }^{212}$ Das Vergütungssystem betrifft im Grunde auch die Abgeltung der allgemeinen teilstationären Leistungen ( $§ 17 \mathrm{~b}$ Abs. $1 \mathrm{~S}$. $3 \mathrm{KHG})$; allerdings gelten für diese angesichts einer gerade hier besonders komplexen Neujustierung der Vergütungsstrukturen noch Sonderregelungen, vgl. $§ 6$ Abs. $1^{213}$ KHEntgG, $\S \S 5$ ff. FPV 2009. 214

Abgesehen von den Wahlleistungen ( $\S 16 \mathrm{ff}$. KHEntgG) sind die Entgelte für allgemeine Krankenhausleistungen für alle Benutzer des Krankenhauses einheitlich zu berechnen, die Fallpauschalen mithin auch für die privat Versicherten maßgeblich (vgl. $\S \S 8$ Abs. 1 S. 1 KHEntgG, $\S 17$ Abs. 1 S. 1 KHG, $\S 14$ Abs. 1 HS. 1 BPflV); dies trifft für Pflegesätze und Vergütungen der vor- und nachstationären Behandlung ebenfalls zu, vgl. § $17 \mathrm{KHG}$.

\subsubsection{Investitionskosten als Vergütungsbestandteil}

In Deutschland besteht seit 1972 ein System der dualen Krankenhausfinanzierung. Investitionskosten werden von der öffentlichen Hand, die behandlungsbezogenen Betriebskosten von den Krankenkassen bzw. den Patienten getragen. ${ }^{215}$ Staatliche Fördermittel allerdings sind weder die einzige Quelle der Investitionsfinanzierung, noch würden sie den tatsächlichen Investitionsbedarf der Krankenhäuser decken. ${ }^{216}$

Je deutlicher Wettbewerb ausgeprägt ist, je größer also der Verantwortungsbereich der Krankenhäuser gezogen und je geringer der staatliche Einfluß ist, desto eher muß

208 S. 2, abrufbar unter www.gesundheitspolitik.net.

209 Eine direkte Bezugnahme kommt in $\S 17$ b Abs. 6 S. 2 Hs. 2 KHG zum Ausdruck, demzufolge ,die auf Grundlage australischer Bewertungen vereinbarten Relativgewichte ... in den folgenden Jahren [nach 2004] auf der Grundlage deutscher Kostenerhebungen zu ersetzen sind.“ Grundlegend zur Systematik der australischen DRG-Klassifikation Tuschen/Trefz, Krankenhausentgeltgesetz (2004), S. $127 \mathrm{ff}$.

210 Im Jahre 2008 waren es noch 1.137, Quelle: GKV-Spitzenverband, G-DRG 2009 nach InEK.

211 Hinzu kommen 127 Zusatzentgelte (2008: 115); Quelle: a.a.O.

212 Näher Knorr/Kraemer, Krankenhausrecht (2005), Einführung, S. XVI.

213 V.a. „für Leistungen, die“, so die Neufassung durch das KHRG, „,noch nicht mit den DRGFallpauschalen und Zusatzentgelten sachgerecht vergütet werden können“.

214 Vgl. etwa $\S \S 6,7$ Abs. 1 S. 2, Abs. 3 HS. 2 FPV 2009.

$215 \mathrm{Zu}$ Einzelheiten unter I.3.4.2.

216 Vgl. unter I.2.2.4. 
das Krankenhaus selbst für die Investitionsmittel aufkommen. Dem vollkommenen Wettbewerb entspräche eine Verknüpfung der Investitionskosten auf der einen mit den Einnahmen bzw. Erlöse zur Vergütung für Leistungen auf der anderen Seite. Ein wettbewerbsorientiertes Vergütungssystem ist danach im Grunde ein eher monistisches System. Der Rückzug der Länder aus der Investitionsförderung allerdings hätte zur Folge, ein regulierendes Steuerungsinstrument aus der Hand zu geben, daß an einer „bedarfsgerechte(n) Versorgung der Bevölkerung mit leistungsfähigen, eigenverantwortlich wirtschaftenden Krankenhäusern" ausgerichtet ist, § 1 Abs. 1, §6 Abs. 1 und § 8 KHG. „Um“ dazu beizutragen, ist „Zweck“ - besser: nachgeordnetes Steuerungsziel des KHG - „die wirtschaftliche Sicherung“ der Krankenhäuser, § 1 Abs. 1 KHG. ${ }^{217}$ Durch die Koppelung der Planbedürfnisse mit der wirtschaftlichen Sicherung und weil gem. § 8 Abs. 2 KHG kein Rechtsanspruch auf Feststellung der Aufnahme in den Krankenhausplan und in das Investitionsprogramm besteht, werden Wettbewerbsanreize, die Investitionsmittel selbst zu erwirtschaften und „Planungssicherheit“ herzustellen, weithin außer acht gelassen. Die Einbindung in wettbewerbliche Nachfragestrukturen stärkte die Investitionsautonomie. Neben der Frage, welche Steuerungsmechanismen dem Staat verbleiben, ist dann allerdings dringend zu beantworten, ob der umschichtungsbedingte „Mehraufwand“ in der direkten Vergütungsfinanzierung damit den Krankenkassen, und über den Beitrag letztlich den versicherten Patienten anzulasten wäre. ${ }^{218}$

Im Zeichen der Einführung des DRG-Systems hat es daher Bestrebungen gegeben, die Investitionskosten durch die Einbeziehung in das Fallpauschalensystem in die Vergütung zu integrieren. Diese waren zunächst gescheitert, ${ }^{219}$ wobei zu vermuten steht, daß neben der Frage der ,zusätzlichen“ Finanzierungslast (durch die Kostenträger) das komplexe Vergütungssystem angesichts nochmals komplexer Übergangsmechanismen, Vereinbarungen und Bewertungsfragen damit stark überfordert (worden) wäre. Zugleich wurde vorausgesehen, sie würden danach erneut zum „Hauptthema“, auch im europäischen Kontext. 220

Wie bereits dargelegt, hat auch das KHRG den entscheidenden Schritt zur Monistik (noch) ${ }^{221}$ nicht gewagt und einen Wandel zum Selektivvertragssystem unter Abschaffung des Kontrahierungszwangs gem. $\S 108$ SGB V 222 nicht vollzogen. Rechtspraktisch vorerst obsolet sind damit auch zwei vormals für diskussionswürdig erachtete Reformüberlegungen in Richtung Monistik:223 zum einen die Verteilung der Investitionsmittel durch die Krankenkassen in Form eines Investitionsprogramms mit Einzelmaß-

\section{Siehe auch I.2.3.1.4.}

218 Siehe auch Neubauer, in: Arnold/Klauber/Schellschmidt (Hrsg.) Krankenhausreport 2002 (2003), S. 71,86 .

219 Näher dazu unter I.3.3.2.

220 Siehe Neubauer, in: Arnold/Klauber/Schellschmidt (Hrsg.) Krankenhausreport 2002 (2003), S. 71 (90).

221 S. unten I.3.4.2.2.

222 Robbers, Geplanter Wettbewerb, das Krankenhaus 2007, S. 207.

223 A.a.O., S. 207 f. 
nahmen, zum anderen - als quasi „echte monistische Finanzierung“ - im Wege eines DRG-Zuschlages. Beide Wege sind - theoretisch - der effektiven Implementierung mit Steuerungswirkung durch Recht zugänglich.

In diesem Sinne konstatierte die Bundesregierung 224 noch vor Inkrafttreten des KHRG in Reaktion auf das Siebzehnte Hauptgutachten 225 der Monopolkommission:

„Eine dauerhafte wirtschaftliche Finanzausstattung der Krankenhäuser könnte auch durch eine monistische Finanzierung gewährleistet werden, bei der der Investitionsbedarf der Krankenhäuser neben den Betriebskosten aus einer Hand finanziert wird. Eine Voraussetzung... wäre jedoch, dass der Bund von den Ländern einen finanzielle Kompensation in Höhe der sich ergebenen Mehrbelastungen erhielte“. Diesbezüglich sei derzeit keine Einigung zu erzielen.

Mit entsprechenden Bestrebungen verbunden wäre die Überlegung, in welchem Verhältnis Planung und Investitionsfinanzierung fortan zueinander stünden und inwiefern die Planung im übrigen die weitgehend flächendeckende und wohnortnahe Versorgung sicherstellen könnte. Insofern ist der regionale Bezug nicht von der Hand zu weisen. Auf der anderer Seite erhebt die Monopolkommission Kritik grundsätzlicher Art an der dualen Finanzierung, nämlich der ökonomischen Problematik der Voraussetzung triftiger Nachfrageprognosen durch staatliche Planung 226 , der Trennung von Kosten- und Ertragseite 227 sowie der Benachteilung von Nichtplankrankenhäusern gegenüber Plankrankenhäusern im Wettbewerb um Privatpatienten. 228

Aus normativer Warte ist der Steuerungsansatz grundsätzlich nicht präjudiziert. Während die dezentrale Verknüpfung von Förderung und Planung tendenziell indirekt und weniger wettbewerbsorientiert ansetzt, verliert sie an verfassungsrechtlich gebotener Rechtfertigungsfähigkeit bezüglich wirtschaftlicher Freiheiten, soweit ein leistungsorientiertes Vergütungssystem ohne eine Finanzierungsbelastung infolge vonÜberkapazitäten die bedarfswirtschaftliche Ausrichtung des Krankenhausrechts entbehrlich macht. ${ }^{229}$ Auf der anderen Seite birgt das monistische System nicht nur die Herausforderung reformpolitisch kaum umsetzbarer beitragsneutraler Umstellung, sondern auch der verantwortungsvollen Organisation der Investitionsfinanzierung in der Verantwortung der GKV bzw. der Gemeinschaft der Kostenträger ${ }^{230}$ bei Sicherstellung bedarfsgerechter Versorgung, ggf. durch zusätzliche regional- und strukturbezogene Investitionsanreize. Eine rein wettbewerbliche Investitionsfinanzierung unter Verzicht auf jede Bedarfsplanung wird staatlicher Gewährleistungsverantwortung kaum gerecht.

224 BT-Drs. 16/11558, S. 10.

225 BT-Drs. 16/10140.

226 A.a.O., S. 323, Ziff. 827.

227 A.a.O., S. 323, Ziff. 829.

228 A.a.O., S. 324, Ziff. 830.

229 S. Lenz, in: ders./Dettling/Kieser, Krankenhausrecht (2007), S. 25 f. Rn. 7 ff.

230 Quaas, in. ders./Zuck, Medizinrecht, 2. Aufl. (2008), S. 535 Rn. 10. 


\subsubsection{Wettbewerb und Planung}

Somit kann es zu einem konfliktträchtigen Aufeinandertreffen von Wettbewerb und Planung kommen, der auch als „ordnungspolitischer Dualismus“ bezeichnet wird. ${ }^{231}$ Dabei ist zu bedenken, daß die durch die Länder verantwortete Investitionsförderung im föderalen System der Bundesrepublik derzeit höchst unterschiedlich ausfällt. ${ }^{232}$ Manche Länder kommen der Investitionsförderung nach verbreiteter Einschätzung nur unzureichend nach, die Finanzierung wird damit zunehmend und tendenziell auf eine ,monistische“ reduziert. ${ }^{233}$ Dies erklärt auch den Befund eines „finanzpolitischen Dualismus zwischen dualistischer und monistischer Finanzierung". 234 Dadurch kann es zu Wettbewerbsverzerrungen kommen. Für die Krankenkassen besteht ein Kontrahierungszwang gegenüber den Plankrankenhäusern. Selbst wenn sich die Länder aus der Investitionsförderung zurückziehen, wird der Status als Plankrankenhaus seitens vieler Krankenhäuser deshalb verteidigt. ${ }^{235}$ So besteht Wettbewerb auch mit Blick auf die Teilhabe an den Planungen der Länder. ${ }^{236}$ Im übrigen werden sich die Länder dagegen stellen, „Investitionsmittel“ in ein monistisches System einzubringen, ohne damit einen maßgeblich Einfluß auf planerisch und infrastrukturell relevante Investitionsentscheidungen zu nehmen. 237

Auf die neu eingeführte Kompetenz zur Entscheidung über die Berechtigung zur ambulanten Behandlung nach § 116b Abs. 2 SGB V durch Bestimmung im Rahmen der Krankenhausplanung wurde bereits hingewiesen. ${ }^{238}$ Die damit verbundene Ersetzung der vormaligen Vertragskompetenz der Krankenkassen durch Integration der Behandlungsbefugnis in den Planungszusammenhang weist ordnungspolitisch zurück in Richtung Regulierung.

\subsubsection{Wettbewerb der Trägerstrukturen und Vertragswettbewerb}

Vor allem im Zeichen neuer Versorgungsformen wird sich ein Vertragswettbewerb um und durch neue Regelungsmodelle entwickeln, die auf der hinzugewonnenen Abschluß- und Gestaltungsfreiheit basieren. Daran allerdings haben die Krankenhäuser

231 Bruckenberger, in: Arnold/Klauber/Schellschmidt (Hrsg.) Krankenhausreport 2002 (2003), S. 93 (101).

232 Vgl. die Abb. bei Bruckenberger, a.a.O., S. 100.

233 S. Neubauer, in: Arnold/Klauber/Schellschmidt (Hrsg.) Krankenhausreport 2002 (2003), S. 71 (77 f.).

234 Bruckenberger, in: Arnold/Klauber/Schellschmidt (Hrsg.) Krankenhausreport 2002 (2003), S. 93 (101).

235 Neubauer, in: Arnold/Klauber/Schellschmidt (Hrsg.) Krankenhausreport 2002 (2003), S. 71 (90).

236 S. unten I.4.3.1. und v.a. II.4.1.

237 Robbers, Geplanter Wettbewerb, das Krankenhaus 2007, S. 207.

238 Unter I.2.1.4. 
(lediglich) ebenso Anteil wie andere Akteure, von der besonderen Bedeutung der Beteiligung an MVZ (oftmals in Form von $\mathrm{GmbH}$ ) ${ }^{239}$ einmal abgesehen.

Um besser zu bestehen und die neuen Möglichkeiten insbesondere der Binnenkooperation der Leistungserbringer besser nutzen zu können, könnte der Wettbewerb zwischen den Krankenhäusern zunehmend auch Ausdruck eines Wettbewerbs um Trägerund Organisationsstrukturen, ${ }^{240}$ eines Wettbewerbs der Gesellschaftsrechte werden. $\mathrm{Da}$ zunehmender Wettbewerb eine besondere „Privatisierungswelle“ präjudiziert, ist nicht zwingend, wenngleich Vorteile im Bereich der „Handlungs- und Entscheidungsfähigkeit, Wirtschaftlichkeit und Investitionskraft" zu würdigen sind, ebenso aber auch Nachteile privater Rechtsformen. ${ }^{241}$

\subsubsection{Qualität und Wettbewerb}

Krankenhäuser stehen in einem Qualitätswettbewerb, der bei stationärer und intensivmedizinischer Behandlung besondere Bedeutung erfährt. Inwiefern „Qualität ein Wettbewerbsparameter im Gesundheitswesen“242 sein kann, unterliegt differenzierter Einschätzung. Qualität ist schwerer zu messen als es Kosten sind, und der Patient wird im Vorfeld einer erstmaligen oder erneuten Behandlung in Anbetracht begrenzter Erfahrungen und Beurteilungskapazitäten eher selten valide Entscheidungshilfen bekommen, um die „Nachfrage“ von Gesundheitsleistungen zu beeinflussen. Hinzu kommt, daß Qualität herkömmlicherweise regulativ gesichert wird. ${ }^{243}$ Ferner besteht die Tendenz, Qualität weniger als Steuerungsfaktor, sondern als begleitendes Ergebnis zu bewerten, und die Furcht, daß Rationalisierungs- und Effizienzwettbewerb unbeschadet regulativer Sicherungsmechanismen zu Qualitätsverlusten führen. Andererseits kann der Innovationswettbewerb Qualität befördern, und im Zeichen eines Spezialisierungswettbewerbs spricht die Menge operativer Routineeingriffe durchaus für einen positiven $\mathrm{Zu}$ sammenhang mit dem Ergebnis. ${ }^{244}$

Ein entscheidender Ansatz lautet daher: Qualitätstransparenz. So wurde im Zuge des Fallpauschalengesetzes eine Regelung geschaffen (vgl. § 137 Abs. 3 S. 1 Nr. 4 SGB V), der zufolge ab $2005^{245}$ alle zwei Jahre von allen zugelassenen Krankenhäusern Quali-

239 Während die GmbH als Rechtsform in den Gesetzesmaterialien erwähnt ist, gilt dies für die GmbH \& Co. KG nicht, bei der nicht nur die primär an medizinischen Vorgaben orientierte Führung tendenziell in Frage steht, sondern zumeist auch die Vereinbarkeit mit dem Berufsrecht nicht gewährleistet sein dürfte, vgl. SG Köln, Urt. v. 24.10.2008, SA KA 1/07, GesR 2009, S. 89.

240 Ausf. I.3.2. sowie II.4.7.

241 Strehl, in: Arnold/Klauber/Schellschmidt (Hrsg.) Krankenhausreport 2002 (2003), S. 113 (127 f.).

242 Näher de Pouvourville, in: Arnold/Klauber/Schellschmidt (Hrsg.) Krankenhausreport 2002 (2003), S. $175 \mathrm{ff}$.

243 Vgl. die $\S \S 135 \mathrm{ff}$. SGB V.

244 Heller, in: Klauber/Robra/Schellschmidt (Hrsg.) Krankenhausreport 2004 (2005), S. 213 ff.

245 Lütticke/Schellschmidt, in: Klauber/Robra/Schellschmidt (Hrsg.) Krankenhausreport 2004 (2005), S. 197. 
tätsberichte zu veröffentlichen sind. Die Gesetzesbegründung 246 sieht darin „einen geeigneten Ansatz ..., auch den Wettbewerb um eine gute Qualität der medizinischen und pflegerischen Versorgung zu stärken“. In der außenwirksamen Instrumentalisierung „können die Kassenärztlichen Vereinigungen und die Krankenkassen und ihre Verbände“, darüber hinaus gem. § 137 Abs. 3 S. 4 SGB V „,die Vertragsärzte und die Versicherten auf der Basis der Qualitätsberichte nach Nr. 6 auch vergleichend über die Qualitätsmerkmale der Krankenhäuser informieren und Empfehlungen aussprechen“. „Leistungsstarke Krankenhäuser“, so die Einschätzung von Medizinern, „,önnen hier sehr profitieren, eine adäquate Risikoadjustierung vorausgesetzt.“247

Mit dem GKV-WSG wurden zahlreiche zusätzliche Qualitätsvorgaben normiert. Sie betreffen insbesondere das ambulante Operieren nach $\S 115 \mathrm{~b}$ und die ambulante Behandlung nach $\S 116 \mathrm{~b}$ SGB V sowie die Vorgabe von Mindestmengen gem. § 137 Abs. 3 S. 1 Nr. 2 SGB V, deren voraussichtliche Nichterreichung bei planbaren Leistungen dazu führt, daß entsprechende Leistungen nicht erbracht werden dürfen (Abs. $3 \mathrm{~S}$. 2). Maßgeblich sind die Festlegungen des diesbezüglich kompetenten Gemeinsamen Bundesausschusses (GBA), die Richtlinienstatus erhalten. ${ }^{248}$

\subsection{Kostenbegrenzung und-kontrolle}

Kosten einzudämmen und zu senken ist seit jeher zentrales Anliegen und Angriffspunkt normativer Steuerung im Gesundheitswesen. Diesem Ziel verpflichtet erweist sich die zunehmende Verankerung wettbewerblicher Elemente im Krankenhauswesen in historischer Perspektive als insgesamt eher junger konzeptioneller Ansatz, der herkömmliche Mechanismen traditioneller Kostendämpfungspolitik ergänzt bzw. ablöst. Das Bemühen um Kostenkontrolle, das dem sozialstaatlich und -politisch geprägten Handlungsauftrag folgt, ein leistungsfähiges und kostengünstiges Krankenhaussystem zu schaffen und zu erhalten, 249 welches die Stabilität der Beitragssätze nicht gefährdet, zieht sich wie ein roter Faden durch die Geschichte der Krankenhausfinanzierung. ${ }^{250}$ Kostendämmung war und bleibt Ziel und Ansatzpunkt normativer Steuerung, und in einem Umfeld vorwiegend dualistischer Finanzierungsströme eine überaus komplexe Aufgabe.

Im wettbewerblich strukturierten System eigenständiger Verantwortungsbereiche vermag der Gesetzgeber die Akteure einzubinden und ihnen direkt oder indirekt die

246 BT-Drucks. 14/6893, S. 31.

247 Schrappe, in: Klauber/Robra/Schellschmidt (Hrsg.) Krankenhausreport 2004 (2005), S. 17 (20)

248 Vgl. auch den Beitrag „GKV-WSG: Darstellung der krankenhausrelevanten Regelungen“ (ohne Autor), das Krankenhaus 2007, S. 199 (203).

249 Vgl. auch $\S 1$ Abs. 1 des KHG, das, „die wirtschaftliche Sicherung der Krankenhäuser“ verfolgt, „um eine bedarfsgerechte Versorgung der Bevölkerung mit leistungsfähigen, eigenverantwortlich wirtschaftenden Krankenhäuern zu gewährleisten und zu sozial tragbaren Pflegesätzen beizutragen."

250 Näher dazu unter I.3.3.2. 
Aufgabe der Kostenkontrolle bereichsspezifisch zuzuweisen. Effizienz wird dabei zum Grundpfeiler der Existenz des Akteurs, v.a. des Leistungserbringes, denn Wettbewerb um Vergütungsanteile, Innovation und Spezialisierung führt auch zu einem Verdrängungswettbewerb. Es ist Ausdruck des Wettbewerbs, daß alle Akteure ,in ein umfassendes Anzreizsystem integriert werden“,251 auch zur Kostensenkung im Krankenhauswesen.

Wettbewerbsorientierte Kostensenkung findet daher in einem multipolaren Beziehungsgeflecht mit spezifizierten, gleichwohl beschränkten Handlungsspielräumen statt. Fallpauschalen verlangen nach Rationalisierung und erhöhen den Druck auf die Krankenhäuser, Sach- und Betriebs- sowie Personalkosten zu senken, ggf. ihre Binnen-, Organisations- oder Kooperationsstrukturen zu ändern. Krankenkassen sind bestrebt, durch den Abschluß neuer, kostengünstiger Versorgungsverträge Effizienzgewinne zu erzielen. Überdies erweist sich Effizienz in der Kostenstruktur anhand der für die Investitionsförderung maßgeblichen zukunftsgerichteten Entscheidungen.

\subsubsection{Leistungsausgaben für die Krankenhausbehandlung in der GKV}

Rund 90\% der bundesrepublikanischen Bevölkerung sind gesetzlich, ${ }^{252}$ etwa $10 \%$ privat krankenversichert. ${ }^{253}$ In der GKV entfielen im Jahre $2008 € 52,57 \mathrm{Mrd}$. und damit 32,7\% der GKV-Gesamtausgaben in Höhe von $€ 160,76$ Mrd. allein auf die Krankenhausbehandlung. ${ }^{254}$ Bezogen auf die Leistungsausgaben ${ }^{255}$ liegt der Anteil bei $34,7 \%$.

Ausgaben für die Krankenhausbehandlung markieren den mit Abstand größten Posten der Ausgaben der GKV. Im zweistelligen Bereich rangieren darüber hinaus nur die Ausgaben für Arzneimittel (18,2\%) und die ärztliche Behandlung (15,1\%). ${ }^{256}$ Ausgaben für Vorsorge- und Rehabilitationsleistungen durch eigenständig kategorisierte (§ 107 Abs. 2 SGB V) und erfasste Vorsorge- und Rehabilitationseinrichtungen sind in den Ausgaben für die Krankenhausbehandlung nicht enthalten. Mit $€ 2,44$ Mrd. werden diese statistisch ebenso getrennt erfasst wie die Vorsorge- und Rehabilitationseinrichtungen ( $§ 111$ SGB V) selbst. ${ }^{257}$ Bezogen auf das einzelne Mitglied stiegen die Ausga-

251 S. Gerlinger, Zwischen Korporatismus und Wettbewerb: Gesundheitspolitische Steuerung im Wandel, Veröffentlichungen der Arbeitsgruppe Public Health, Wissenschaftszentrum Berlin für Sozialforschung (2002), S. 7.

252 BMGS (Hrsg.), Ratgeber zur gesetzlichen Krankenversicherung Teil 1 (2005), S. 19.

253 Der Verband der PKV ermittelt für 2007 eine Zahl von 10,40\% (private Vollversicherung), vgl. den Zahlenbericht 2007/2008, S. 27.

$254 B M G$, Gesetzliche Krankenversicherung - Kennzahlen und Faustformeln, 1.-4. Q. 2008, Stand: 4.3.2009, Ziff. 2; abrufbar unter www.bmg.bund.de (Statistiken, Gesetzliche Krankenversicherung).

255 Unter Abzug der Netto-Verwaltungskosten von $€$ 8,29 Mrd. und damit ausgehend von $€ 151,12$ Mrd., a.a.O.

256 Ohne Dialysesachkosten und Soziotherapie, a.a.O.

257 Vgl. unter I.3.3.2. 
ben für die Krankenhausbehandlung im Jahr 2007 auf erstmals knapp über $€ 1.000$ an. 258

\subsubsection{Leistungsausgaben für die Krankenhausbehandlung in der PKV}

In der Bundesrepublik verfügten Ende 2007 rund 8,54 Mio. Personen über eine private Krankheitskostenvollversicherung, die per Definition allgemeine Krankenhausleistungen einschließt. ${ }^{259} 20$ Mio. Personen 260 sind (nach gesonderter Zählung) zusatzversichert; 2006 waren es noch 18,4 Mio., ${ }^{261}$ im Jahre 2003 nur 14,69 Mio. Personen. ${ }^{262}$ Krankenhausspezifisch hatten 5,16 Mio. Personen eine Versicherung für Wahlleistungen (§ 17 KHEntgG) im Krankenhaus abgeschlossen. ${ }^{263}$ Die Zahl der 2007 privat Vollversicherten mit Wahlleistungen im Krankenhaus lag bei 7,2 Mio., derjenigen mit Krankenhaustagegeldversicherung bei 2 Mio. Personen. 264

$€ 5,55$ Mrd. und damit 30,33\% der Leistungsausgaben von $€ 18,89$ Mrd. in der PKV im Jahre 2007 entfielen auf den als „stationäre Leistungen“ erfassten Krankenhausbereich. 265 Von den Ausgaben für stationäre Leistungen entfielen $€ 2,80 \mathrm{Mrd}$. auf allgemeine Krankenhausleistungen; $€ 2,09$ Mrd. wurden für die Wahlleistung „Arzt“, $€ 519$ Mio. für die Wahlleistung „Unterbringung“ ausgegeben. ${ }^{266}$

Bezogen auf das Jahr 2005 gingen die Leistungsausgaben im Bereich der Wahlleistungen Unterkunft (-3,21\%) und Ersatz-Krankenhaustagegeld (-7,2\%) deutlich zurück, stiegen im Bereich der allgemeinen Krankenhausleistungen indes mit 7,11\% deutlich höher an als im Vergleich zur GKV (2,8\% bzw. $+3,3 \%$ je Mitglied im Jahre 2005). ${ }^{267}$ Dem entsprach eine Reduktion der Erlöse der Krankenhäuser in den alten Bundeslän-

258 BMG, Gesetzliche Krankenversicherung - Kennzahlen und Faustformeln, Stand: 4.3.2009, Ziff. 4; abrufbar unter www.bmg.bund.de (Statistiken, Gesetzliche Krankenversicherung).

259 Zahlenbericht 2007/2008, S. 27; der Anteil der Krankheitsvollversicherung, der Hauptversicherungsart der PKV, an den gesamten Beitragseinnahmen beträgt 71,99\%. Die im Sinne der PKV positive Differenz zwischen den Übertritten zur PKV und den Abgängen zur Pflichtversicherung im Rahmen der Krankheitsvollversicherung betrug im Jahre 2007 nur 79.000 (2006: 140.800), a.a.O.; 2005: 120.300 (2004: 167.100; 2003: 208.000); a.a.O., S. 25 (2005/2006); S. 14 (2004/2005).

260 Doppelzählungen ergeben sich u.a. daraus, daß auch Versicherte mitgezählt werden, die Krankheitsvollversicherung und Zusatzversicherung bei verschiedenen Unternehmen abgeschlossen haben, Zahlenbericht der PKV 2007/2008, S. 33.

261 A.a.O.

262 Zahlenbericht der PKV 2004/2004, S. 12, 17.

263 Zahlenbericht 2007/2008, S. 33.

264 A.a.O., S. 29.

265 A.a.O., S. $51 \mathrm{f}$.

266 A.a.O., S. 49. Hinzu kommen $€ 56,7$ Mio. für Ersatz-Krankenhaustagegeld und $€ 77,4$ Mio. für Sonstiges. Über die Abrechnung der Wahlleistung „Unterkunft“ haben die Unternehmen der privaten Krankenversicherung mit mehr als 66\% der Krankenhäuser Verträge abgeschlossen, vgl. Deutsches Krankenhausinstitut (Hrsg.), Krankenhaus Barometer, Umfrage 2005, S. 17.

267 Vgl. BMG, Gesetzliche Krankenversicherung - Kennzahlen und Faustformeln, Stand: 18. August 2008, abrufbar unter www.bmg.bund.de (Statistiken, Gesetzliche Krankenversicherung). 
dern aus der Wahlleistung „Unterkunft“ von 2000 bis 2004 um rund ein Viertel,268 während der durchschnittliche Erlös für die Wahlleistung „Arzt“ je Krankenhaus (alte und neue Länder) gegenüber 2000 um 23,3\% auf $€ 827.223$ angestiegen ist.

Bezogen auf die folgenden Jahre stiegen die Erlöse aus der Wahlleistung Unterkunft wiederum um jeweils mehr als $6 \%$ gegenüber dem Vorjahr an, ${ }^{269}$ zuletzt mit einer Steigerung der Aufwendungen für allgemeine Krankenhausleistungen mit 3,5\% gegenüber dem Vorjahr im Jahre 2007. Die zuletzt (2007) ähnlich hohe Ausgabensteigerung in der GKV fiel im Jahr zuvor kaum spürbar aus.

\subsubsection{Kosten der Krankenhäuser}

Von den Leistungsausgaben der GKV und PKV zu unterscheiden sind die Gesamtkosten der Krankenhäuser und ihrer Ausbildungsstätten. Sie werden auf der Grundlage der Krankenhausstatistikverordnung (KHStatV) 270 i.V.m. dem Bundesstatistikgesetz (BStatG) erhoben. Die seit 2002 nach dem Bruttoprinzip ermittelten Gesamtkosten für das Jahr 2007 betrugen $€ 68,1$ Mrd. mit einem Personalkostenanteil von rund $€ 42 \mathrm{Mrd}$. Die um den nichtstationären Anteil bereinigten Kosten ${ }^{271}$ lagen bei $€ 60,4 \mathrm{Mrd}$. Dies bedeutet eine Kostensteigerung gegenüber dem Vorjahr um 4,1\%, wobei die Fallzahl um 2,1\% zunahm, die Verweildauer um 0,2 Tage zurückging und die Zahl der Einrichtungen um 0,8\% abnahm. ${ }^{272}$ Die durchschnittlich bereinigten Kosten pro Krankenhaus stiegen sogar um 4,9\% auf $€$ 28,6 Mio.; im Jahre 1991 betrugen die Gesamtkosten noch $€ 37,4$ Mrd. insgesamt bzw. $€ 15,5$ Mio. je Krankenhaus. Gut $60 \%$ der Personalkosten im Jahre 2007 entfielen auf den ärztlichen und den Pflegedienst. Knapp die Hälfte Sachkosten in Höhe von $€ 25,7$ Mrd. entfiel auf den medizinischen Bedarf, wobei die Arzneimittel mit $€ 2,9$ Mrd. den größten Anteil ausmachten. 273

\subsubsection{Beteiligung der Krankenhäuser an finanziellen Stabilisierungsmaßnahmen}

\subsubsection{Rechnungskürzungen mit Finanzierungsfunktion (Anschubfinanzierung IV)}

Anreizfunktion für den Abschluß von IV-Verträgen entfaltete bis einschließlich 2008 das Instrument der so genannten Anschubfinanzierung, § 140d SGB V. Da die Mittel

268 Dies ist sowohl einem Rückgang der Leistungen als auch einem Rückgang je Wahlleistungstag geschuldet, siehe DKI (Hrsg.), Krankenhaus Barometer, Umfrage 2005, vgl. S. 18 f.

269 Zahlenberichte 2006/2006 und 2007/2008, jeweils S. 51.

270 In der für das Berichtsjahr gültigen Fassung, vgl. die Erläuterungen zum Kostennachweis in StBA (Hrsg.), „Kostennachweis der Krankenhäuser“ 2007 (2008).

271 Diese können im Gegensatz zu einzelnen Kostenarten über die Jahre hinweg durchgängig verglichen werden. Es handelt sich um die Gesamtkosten unter Abzügen für wissenschaftliche Forschung und Lehre, für die Ambulanz sowie sonstige Abzüge (v.a. vor- und nachstationäre Behandlungen, ärztliche und nichtärztliche Wahlleistungen sowie belegärztliche Leistungen), vgl. Kostennachweis 2005, Erläuterungen.

272 StBA, Grunddaten der Krankenhäuser 2007 (2008), Allgemeiner Überblick 2007, sowie Ziff. I. 1.1.

273 StBA (Hrsg.), „Kostennachweis der Krankenhäuser“ 2007 (2008), Allgemeiner Überblick 2005. 
der Anschubfinanzierung allerdings aus Kürzungen der an die Kassenärztlichen Vereinigungen zu entrichtenden Gesamtvergütung sowie aus Kürzungen der Rechnungen der Krankenhäuser in Höhe von jeweils 1\% aufzubringen waren, korrespondiert mit der Anreizfunktion ein zusätzlicher Kosten- bzw. ein Druck zur partiellen Refinanzierung, um insgesamt gesehen keine Nachteile bei der Vergütung hinnehmen zu müssen.

Die Anschubfinanzierung wurde zunächst für die Jahre 2004 bis 2006 eingeführt und im Zuge des VÄndG zunächst bis zum 31.12.2007 verlängert. Mit der erneuten Verlängerung durch das GKV-WSG bis Ende 2008 ging - zur Vermeindung von Quersubventionen - eine Begrenzung der Förderung der nach dem 1. April 2007 geschlossenen Verträge auf Leistungen der Krankenhäuser und der Vertragärzte einher, § 140d Abs. 1 S. 2 und 3 SGB V.

Der gesetzlich verankerte Beitrag der Krankenhäuser zur Anschubfinanzierung erfolgte in Form eines kassenseitigen, zweckgebunden Einbehalts in Höhe von bis zu 1\% der Krankenhausrechnungen für voll- und teilstationäre Versorgung, § 140d SGB V. ${ }^{274}$ Bezogen auf das Jahr 2002 bspw. bedeutet dies ein potentielles Finanzierungsvolumen von bis zu $€ 460$ Mio., 275 wobei alsbald ein Streit darüber entbrannte, ob die Berechtigung der Krankenkassen zum - vorläufigen, als faktische Stundung finanzwirksamen Einbehalt von Vergütungsanteilen davon abhängt, daß diese einen Versorgungsvertrag bereits abgeschlossen hat. ${ }^{276}$ Die dahin gehende Auffassung hat sich mittlerweile durchgesetzt. ${ }^{277}$ Soweit die Vertragskosten über die Mittel der Anschubfinanzierung hinausgingen, war die Gesamtvergütung nach $\S 85$ Abs. 2 SGB V nach den Vorgaben des $\S 140 d$ Abs. 2 S. 1 SGB V zu bereinigen.

Damit werden die Krankenhäuser allgemein zur Reformfinanzierung herangezogen, die ihnen langfristig in konkreten Verhältnissen möglicherweise mittelbar in Teilen wieder zugute kommt, die sie aktuell jedoch finanziell belastet. Nach jüngeren Umfragewerten lag die durchschnittliche Rechnungskürzung im Jahre 2005 bei $€ 116.100$ pro Krankenhaus; für Krankenhäuser ab 600 Betten betrug sie $€ 418.200 .278$ Bezogen auf die an der IV teilnehmenden Krankenhäuser lag die Kürzung im Durchschnitt bei $€ 235.000$, der allerdings durchschnittliche Erlöse von $€ 388.000$ gegenüber standen. ${ }^{279}$

Zum 1.1.2009 ist die Anschubfinanzierung endgültig ausgelaufen. Dies steht im Zusammenhang mit der Einführung der morbiditätsorientierten Vergütung in der vertrags-

$274 \S 140 d$ Abs. 1 SGB V. Davon strikt zu unterscheiden sind Rechnungskürzungen bzw. Erstattungen und Abgaben wegen Schlechtleistung, Fehlbelegungen und nicht indizierten Behandlungen (vgl. $\S 17 \mathrm{c} \mathrm{KHG)} \mathrm{etc.}$

275 BT-Drucks. 15/1525, S. 131.

276 Dagegen LSG Brandenburg, Beschl. v. 1.11.2004, abgedruckt in MedR 2005, S. 62 (63) m. krit. Anm. von Bold, a.a.O.; anders das SG Saarland, Beschl. v. 14.12.2004, abgedruckt in: ZMGR 2005, S. $62 \mathrm{ff}$.

277 Siehe Huster, in. Becker/Kingreen, SGB V (2008), § 140d Rn. 1.

278 DKI (Hrsg.), Krankenhaus Barometer, Umfrage 2006, S. 57. Im Jahre 2004 lag sie bei $€ 24.800$ pro Krankenhaus; für Krankenhäuser ab 600 Betten betrug sie $€$ 76.700, Umfrage 2005, S. 48.

279 Vgl. oben I.2.1.3.2. 
ärztlichen Versorgung, vgl. § 87a SGB V. Erst deren Einführung macht eine systemkonsistente Bereinigung des (gesamten) Behandlungsbedarfs (vgl. nunmehr § 87a Abs. 3 S. 2 SGB V) möglich. Eine Bereinigung vor Einführung der morbiditätsorientierten Vergütung hätte entweder zu größeren Verwerfungen oder höchst komplexen Bereinigungsmechanismen geführt.

Gleichzeitig, so ließe sich argumentieren, wird die Gefahr von Verwerfungen bzw. (Wettbewerbs-)Verzerrungen mittels der Aufhebung der Anschubfinanzierung in der integrierten Versorgung beseitigt. So mag die Zahl der Verträge, insbesondere der im Hinblick auf die Anschubfinanzierung geschlossenen oder aus anderen, wie etwa Strukturverträgen heraus „umstrukturierten“, zurückgehen. Erfolg wird jedoch mutmaßlich populationsbezogenen und flächendeckenden bzw. qualitativ und unter dem Gesichtspunkt des Einspar-Contractings optimierten indikationsbezogenen Vertragsmodellen beschieden sein.

\subsubsection{2. „Sanierungsbeitrag“ der Krankenhäuser}

„Um die Krankenhäuser an den finanziellen Stabilisierungsmaßnahmen für die gesetzliche Krankenversicherung zu beteiligen“, der einem Prozent der Ausgaben für stationäre Krankenhausleistungen entsprechen sollte, wurde den Krankenhäusern im Zuge des GKV-WSG ein dreiteiliger „Sanierungsbeitrag“ auferlegt: 280 Erstens erfolgte nach $\S 8$ Abs. 9 KHEntgG eine unmittelbare Kürzung der Krankenhausrechnungen für vollund teilstationäre Leistungen ab dem Jahr 2007, die allerdings zuletzt mit dem KHRG für das Jahr 2009 wieder abgeschafft wurde. Im Jahr 2009 führt die Abschaffung „wieder“ - zu „Mehrausgaben in Höhe von 230 Mio. €“. ${ }^{281}$ Zweitens wurde die Rückzahlungspflicht der Krankenkassen für nicht verwendete Mittel der Anschubfinanzierung für die Integrierte Versorgung, die sich bis 2008 auch aus Rechnungskürzungen für Krankenhäuser speisten, abgeschafft, soweit Mittel in den Jahren 2004 bis 2006 einbehalten wurden, $\S 140$ d Abs. 1 S. 8 SGB V. Drittens wurde die in $\S 4$ Abs. 9 S. 2 KHEntgG verankerte Mindererlösausgleichsquote 282 von grundsätzlich $40 \%$ auf $20 \%$ ab dem Jahre 2007 abgesenkt.

Der Sanierungsbeitrag ist nach Auffassung der bisher vorliegenden sozial- und landessozialgerichtlichen Rspr. ${ }^{283}$ verfassungsgemäß. Der Eingriff in die Berufsfreiheit sei durch vernünftige Gründe des Gemeinwohls ebenso gerechtfertigt, wie der Rechnungsabschlag - Rabattverpflichtungen vergleichbar - nicht abgabengleich, sondern „der Ka-

280 Zu Terminologie und Bestandteilen BT-Drs. 16/3100, S. 194.

281 Rau, Regelungen des Krankenhausfinanzierungsreformgesetzes, das Krankenhaus 2009, S. 198 (201).

282 Ausgleich für Mindererlöse infolge einer hinter dem vereinbarten Budget zurückbleibenden Leistungsmenge.

283 Vgl. insbesondere $S G$ Aachen, S 13 KR 41/08 (s. auch die dortigen Hinweise zur gegenteiligen Auffassung), S 13 KR 118/07 bis 120/07, ebenso bestätigt durch $L S G N R W$, L 16 KR 87/08, 88/08 und 18/09 wie ein Urteil des SG Dortmund (Pressemitteilung, 26.3.2009). 
tegorie staatlicher Preisreglementierungen zuzuordnen“. Weitere Rechtsschutzersuchen einschließlich der Anrufung von $B S G$ und $B V e r f G$ sind angekündigt bzw. stehen zu erwarten.

\subsubsection{Investitionen}

In wettbewerblichen Wirtschaftsstrukturen hängt der Erfolg unternehmerisch handelnder Akteure wesentlich von den Investitionen, speziell von Rationalisierungs- und Innovationsinvestitionen ab. ${ }^{284}$ Aus volkswirtschaftlicher Sicht tragen vor allem „kapazitätserweiternde Neu-Investitionen“ zum Stand der Medizin bei. 285

Im Jahr 2005 betrug die Investitionsfinanzierung der Länder gem. § 9 KHG einer Bestandsaufnahme der $D K G$ zufolge $€ 2,7$ Mrd. ${ }^{286}$ Gegenüber 2004 ist ein Rückgang um 4,6\% zu verzeichnen. 287 Der Rückgang der Investitionsfinanzierung des Jahres 2003 gegenüber dem Vorjahr beträgt nominal 11,75\%.288 Der Rückgang der Fördermittel zwischen 1991 und 2003 betrug mit $€ 797$ Mio. 21,9\% (real rund 38,8\%);289 der Anteil am Bruttoinlandsprodukt sank von 0,24\% auf 0,13\% im Jahre 2003 bzw. 0,12\% im Jahr 2005. 290

Rund $67 \%$ der Investitionsmittel sind Fördermittel des Landes, davon 47\% aus Pauschal-, 20\% aus Einzelfördermitteln. ${ }^{291}$ Die Krankenhäuser steuern 20,4\% aus Eigenmitteln bei, der Träger fördert in Höhe von 5,5\%. Die Erwartung der Krankenhäuser im Hinblick auf die künftige Bereitstellung von Investitionsmitteln ist skeptisch. ${ }^{292} \mathrm{Zu}$ nehmend wird der Versuch unternommen, private Investoren im Wege vielgestaltiger Modelle des Public Private Partnership (PPP) einzubinden, ${ }^{293}$ d.h. nach Wegen der (vorwiegend funktionellen) (Teil-) Privatisierung, z.B. nach Betreiber-, Konzessions-, Herstellungs- und Leasingmodellen zu suchen. Die Bau- und Anlagenfinanzierung nimmt nach Umfragewerten gleichwohl einen geringen Anteil an PPP-Projekten ein. ${ }^{294}$ Je nach Ausgestaltung der ,partnerschaftlichen Investitionslösung“ können PPP und

284 Neubauer, in: Arnold/Klauber/Schellschmidt (Hrsg.) Krankenhausreport 2002 (2003), S. 78.

285 Neubauer, a.a.O., S. 79.

$286 D K G$, Bestandsaufnahme zur Krankenhausplanung und Investitionsfinanzierung in den Bundesländern, Stand Januar 2006, S. 57; abrufbar unter ww.dkgev.de (Downloads, Finanzierung \& Planung), 4.1.2006.

287 A.a.O.

$288 D K G$, Bestandsaufnahme zur Krankenhausplanung und Investitionsfinanzierung in den Bundesländern, November 2004, S. 37; abrufbar unter ww.dkgev.de (Downloads, Finanzierung \& Planung), 24.11.2004.

289 A.a.O.

$290 D K G$, Bestandsaufnahme zur Krankenhausplanung und Investitionsfinanzierung in den Bundesländern, Stand Januar 2006, S. 51; abrufbar unter ww.dkgev.de (Downloads, Finanzierung \& Planung), 4.1.2006.

291 DKI, Krankenhaus Barometer Umfrage 2005, S. 25.

292 A.a.O., S. $28 \mathrm{ff}$.

293 A.a.O., S. 30.

294 A.a.O. 
Förderrecht durchaus kompatibel sein und einander ergänzen, nicht zuletzt vor dem Hintergrund einer förderrechtlichen Privilegierung von Ausgliederungen oder der ausdrücklichen Aufnahme einer Teilförderung in das Förderrecht. ${ }^{295}$ Der Kanon der förderrechtlich zulässigen PPP-Modelle orientiert sich insbesondere daran, ,dass die Fördermittel auch langfristig zweckentsprechend zur Finanzierung einer Krankenhausinvestition eingesetzt werden“. ${ }^{296}$ Bereits im Koalitionsvertrag von 2005 wurde das Modell des PPP beschworen und kritisiert, daß das KHG insofern Hindernisse bereite. ${ }^{297}$

Vor allem die staatliche, rückläufige Förderung wird dem tatsächlichen Investitionsbedarf im Zeichen anhaltender Kostensteigerungen und medizinischem Fortschritt nach überwiegender Auffassung insgesamt nicht gerecht. ${ }^{298}$ Im Zeichen wettbewerbsorientierter Restrukturierung und Spezialisierung wird der Investitionsbedarf möglicherweise noch deutlich ansteigen. Gleichwohl ist ein Rückgang der öffentlichen Investitionstätigkeit zu verzeichnen, Indikator nicht ausreichender Investitionsförderung. ${ }^{299}$ Ein Vergleich der Anteile der Investitionen an den Anschaffungswerten der Krankenhäuser seit 2002 weist eine negative Entwicklung aus. ${ }^{300}$ Die Kennzahl sank von 15,3\% im Jahre 2002 auf 13,6\% im Folgejahr und schließlich 11,3\% im Jahre 2004. In Relation zu den Erlösen aus Krankenhausleistungen betrug die Quote der Investitionen im Jahre 2004 im Durchschnitt 10,5\%. ${ }^{301}$ Bereits auf der Grundlage der volkswirtschaftlichen Bruttoinvestitionsquote von 21,3\% im Jahre 1999 wurde eine Investitionslücke von jährlich $€$ 10.9 Mrd. ermittelt. 302

Die Krankenhäuser investieren nach der Umfrage 2005 des DKI im Durchschnitt rund $42 \%$ der Mittel für die Einrichtung der Krankenzimmer und Behandlungsräume und $27,4 \%$ in Grundstücke und Gebäude. ${ }^{303}$ Auf Software und EDV-Technik ${ }^{304}$ entfal-

295 So für Bayern Knorr, das Krankenhaus 2007, S. 743 (745).

296 Knorr, a.a.O., S. 743 (747) und zu den konkreten Modellen S. 746.

297 www.cducsu.de/upload/koavertrag0509.pdf, B I. 1.2.

$298 D K G$, Bestandsaufnahme zur Krankenhausplanung und Investitionsfinanzierung in den Bundesländern, November 2004, S. 37 (40), ww.dkgev.de (Downloads, Finanzierung \& Planung), 24.11. 2004, unter Verweis auf das Gutachten „Finanzierung der Investitionen und der Instandhaltung von Krankenhäusern durch Nutzungsentgelte“, hrsg. vom Institut für Funktionsanalyse im Gesundheitswesen, Hamburg 2000, abrufbar unter www.bmg.bund.de (unter Themenschwerpunkte/Ressortforschung, Kurzberichte Archiv, 16.3.2000. Eine empirische Bedarfsanalyse ist diesem jedoch nicht zu entnehmen; stattdessen wird die relative Stagnation untermauert bzw. die Aussage getroffen, ein Investitionsrückstau könne durch Instandhaltungsquoten, die zudem dynamisch fortzuschreiben seien, kaum ausgeglichen werden, vgl. z.B. S. 137, S. 143 f.

299 DKI, Krankenhaus Barometer Umfrage 2005, S. 25.

300 A.a.O., S. 23.

301 A.a.O., S. 24.

302 Neubauer, in: Arnold/Klauber/Schellschmidt (Hrsg.) Krankenhausreport 2002 (2003), S. 71, 77 f. gemessen am Ausgaben- bzw. Erlösvolumen für Krankenhausleistungen von 67,8 Mrd. €, angesichts eines Fördervolumens von $€$ 3,5 Mrd.

303 DKI, Krankenhaus Barometer Umfrage 2005, S. 26 f.

304 Mit zunehmender Bettengrößenklasse nimmt der Anteil der Ausgaben für Hard- und Software (22\% bei Häusern unter 100 Betten) stark ab ( $8,9 \%$ bei Krankenhäusern über 600 Betten). 
len 7,8 bzw. 6,3\%, auf technische Betriebsanlagen 5,4\% und 10,5\% auf sonstige Bereiche (Wirtschafts- und Verwaltungsbedarf, Anlagen im Bau). Nach der Umfrage (DKI) Anfang 2009, die im Kontext der Diskussion um die Bereitstellung von Investitionsfördermitteln des „Konjunkturpaktes II“ und in Anknüpfung an die Ergebnisse der Prognos-Studie ${ }^{305}$ zugunsten besonders positiver makroökonomischer Auswirkungen zusätzlicher Investitionen erstellt wurde, betrug im Jahr 2009 der Bedarf an realisierund abschließend durchführbaren Investitionen $€ 7,38$ Mrd. ${ }^{306}$

\subsection{Normative Steuerung und die politische Diskussion}

\subsubsection{Normative Steuerung}

Der Begriff „Steuerung“ lässt sich - unbeschadet seiner Verankerung in der Soziologie - auch juristisch erfassen, v.a. funktional als Verhaltenssteuerung durch Recht. § 62 Abs. 5 SGB V spricht von der (Evaluation der) „Steuerungswirkung“ der „Ausnahmeregelungen von der Zuzahlungspflicht““.307 Auch im Rahmen des § 87 Abs. 7 SGB V geht es um Beurteilungen hinsichtlich der Steuerung des Niederlassungsverhaltens durch Zulassungsbeschränkungen. Der Begriff wird positiv meist dann verwendet, wenn es um das $\mathrm{Ob}$ oder die Beurteilung von ergriffenen Maßnahmen geht. I.ü. wird Verhaltenssteuerung oftmals mittels ,,indirekter“ Wirkungsweisen durch Anreiz- und Kontrollelemente verfolgt. Dabei ist u.U. ein hohes Maß an finanzieller Transparenz vonnöten, damit „der Gesetzgeber seine Steuerungsfunktion sachgerecht wahrnehmen“ kann. ${ }^{308}$

Die Konzeptualisierung juristisch fundierter Anreizstrukturen und der dabei erkennbaren Interdependenzen sollte angesichts dessen systematisch begriffen und nach Verantwortungsträgern, Adressaten, Steuerungsrichtung, Einsatz rechtlicher Mittel sowie wirkungsspezifisch konturiert werden. Sie ist mehr- und vielschichtig und zeichnet sich nicht nur dadurch aus, daß Verhaltensweisen und Verhältnisse der Akteure (einschließlich der staatlichen) erfaßt werden, die Vorgaben umsetzen und vollziehen, die gestalten und entscheiden. Darüber hinaus befördern Strukturprinzipien wie das Selektivvertragsprinzip einen Wettbewerb der Vertrags- und Organisationsmodelle, welche ihrerseits zu direkten Reformoptionen avancieren oder aber Korrekturbedarf illustrieren.

Steuerungseffekte sind schwer zu messen. Wichtig ist daher, die für die multipolaren Wettbewerbsbeziehungen im Kausalitätsgeflecht maßgeblichen Bausteine zu identifizieren, die eine Annäherung ermöglichen, um unter II. die marktorientiert normativ induzierte Verhaltenssteuerung zu begreifen. Dabei sind konterkarierende Effekte ebenso einzubeziehen wie eine Verflechtung verschiedenster juristischer Handlungsformen und

305 Zusammenfassung unter www.dkgev.de; Zugriff: Jan. 2009.

306 DKI, Kurzfristige Investitionsmaßnahmen der Krankenhäuser im Jahr 2009, S. 2 ff.

307 S. dazu Sichert, in: Becker/Kingreen, SGB V (2008), § 62 Rn. 28.

308 Vgl. die Begründung zum GKV-OrgWG, BT-Drs. 16/9559, S. 1 (15). 
Verbandskompetenzen. Eine (da und dort) vermutete Steuerungsresistenz ${ }^{309}$ jedenfalls hindert das Projekt nicht und ließe sich möglicherweise gar bereichsspezifisch identifizieren. Gleichzeitig gilt es, zwischen zielorientierter und reformfolgeninduzierter Steuerung zu unterscheiden, wobei letztere insbesondere auf die Abwehr von „Fehlanreizen“ oder Reaktion auf Fehlentwicklungen gerichtet ist (,Nachsteuern“).

Der Focus im Bereich normativer Steuerung negiert keinesfalls die Steuerungswirkung, die von (innovativen) unternehmenspolitischen und personalwirtschaftlichen Ansätzen ausgeht. Auch diese können im Zuge einer strukturellen Weiterentwicklung zur Konzernpolitik bzw. Governance quasi-normative Qualität erlangen. Die Wirkung normativer Steuerung vollumfänglich zu messen, hieße danach auch, solchermaßen wechselbezügliche Kausalitätsbeziehungen und vollumfänglich gewichtend zu identifizieren. Dies allerdings kann aus juristischer Warte (allein) kaum geleistet werden, wohingegen der Einfluß normativer Steuerung auf (die Entwicklung) managementorientierter Konzepte durchaus aufscheint.

\subsubsection{Normative und administrative Steuerung}

Im Mittelpunkt der Untersuchung steht die normative Steuerung durch den formellen Gesetzgeber. Das bedeutet jedoch nicht, daß administrative Entscheidungen im Vollzug und in der Interpretation normativer Vorgaben prinzipiell von untergeordneter Bedeutung sind. Mitunter wegweisend sind bspw.Entscheidungen des BKartA betreffend die marktorientierte Interpretation der Wettbewerbsverhältnisse im Zusammenspiel von Wettbewerbs- und (tradiertem) Gesundheitsrecht. Steuerung kann danach auch unter dem Gesichtspunkt autoritativer Entscheidung erfolgen. Im Lichte ihrer unmittelbaren Gesetzesbindung unterscheidet sich dabei die öffentlich administrative von der konzernpolitischen „Governance“.

\subsubsection{Steuerung durch den formellen Gesetzgeber (Verantwortungsträger)}

Im föderalen System bundesstaatlicher Ordnung wundert es nicht, daß der Verteilung der Gesetzgebungszuständigkeiten folgend mehrere Verantwortungsträger agieren. Durch KHG und KHEntgG etwa steuert der Bund in konkurrierender Gesetzgebung (Art. 74 Abs. 1 Nr. 11 und 19a GG) durch Vorgaben für die Krankenhausfinanzierung, während den Ländern als weiteren Verantwortungsgträgern die Planung und Investitionsförderung obliegt.

309 Vgl. Höfling, Vom Krankenhausrecht zum Krankenhausregulierungsrecht, GesR 2007, S. 289 (295): „Als selbstreferenzielles Teilsystem erzeugt das Gesundheitssystem nicht nur seine Strukturen, sondern auch die Elemente, aus denen es besteht, fortlaufend selbst [unter Berufung auf Luhmann]. Dabei ist es prinzipiell von außen nicht steuerbar. Wenn allerdings diese Annahme zutrifft und das Krankenhaus- und Gesundheitssystem geradezu immanent gegenüber der Politik steuerungsresistent sind, dann wäre es allerdings auch für eine sich als anwendungsorientierte Steuerungswissenschaft verstehende Rechtswissenschaft sinnlos, sich des Themas anzunehmen. Aber die... Fragen betreffen in einer so existenziellen Weise die Zukunftsfähigkeit des sozialen Rechtsstaates, daß man sich eine derartige Resignation nicht erlauben kann.“ 


\subsubsection{Doppelstellung der Länder als Adressaten und Verantwortungsträger}

Zugleich sind die Länder nach Systematik und Inhalt der $\S \S 6$ ff. KHG Adressaten der Steuerung, wenn man eine Betrachtungsweise gelten lässt, der zufolge sich erstens Steuerungsketten identifizieren lassen, und diese zweitens den nachgeordneten Verantwortungsträgern ihrerseits Steuerungsspielräume einräumen.

\subsubsection{Steuerungsziele}

Wie mit Blick auf das KHG angedeutet, können Steuerungsziele mehrschichtig sein, d.h. ein spezifisches Ziel durch Wettbewerb verfolgen oder die Intention, Wettbewerb als Eigenwert zu verwirklichen. Es bietet sich daher an, zwischen Wettbewerb als Ziel und Mittel sowie zwischen primären und sekundären Zielsetzungen zu unterscheiden, vgl. § 1 Abs. 1 KHG. Dem entspräche eine Einordnung in das „Zielsystem der GKV“, die - u.a. - zwischen „Leistungszielen“ und ,politischen Nebenzielen“ unterscheidet. 310

Soweit mehrere Ziele verfolgt werden, sind Zielkonkurrenzen möglich, die ggf. in Form von Zielhierarchien bzw. im Wege spezifischer Konkurrenzregeln gelöst werden müssen. Auch die Konkurrenz verschiedener Rechtsregime, etwa des Wettbewerbs- und Kartellrechts auf der einen sowie des Sozialrechts auf der anderen Seite, vgl. § 69 SGB $\mathrm{V}$, bringen dies zum Ausdruck. Schließlich werden neue Versorgungsformen eingesetzt, um kostengünstige Versorgung und einen niedrigen Beitragssatz sicherzustellen, Wettbewerb nachhaltig zu implementieren, aber bspw. auch, um „Strukturinnovationen durch Integrierte Versorgung in den Neuen Bundesländern" zu erzielen. ${ }^{311}$

Zielkonflikte sind mithin nicht ausgeschlossen. Je ausgereifter Steuerungskonzepte und je dezidierter diese Steuerung durch den oder die Normgeber indes abgestimmt sind, desto seltener treten Konflikte auf. Der BGH stellte Anfang 2008 in einem Beschluß zur Fusionskontrolle fest, es bestehe „Zielkongruenz zwischen der Fusionskontrolle und der Gesundheitspolitik". ${ }^{112}$ Dies indes muss nicht der Fall sein. Dann aber ist Recht herausgefordert, Konflikte zu lösen, sei mit Blick auf die Einheit der Rechtsordnung, bei Fragen zu „Bereichsausnahmen“ 313 oder in Form der methodenorientierten Lösung klassischer Normkonflikte.

\subsubsection{Adressaten (I) - individuelle Leistungserbringer und Kostenträger}

Neben den in einer Doppelstellung befindlichen Ländern sind v.a. die Leistungs-, aber auch die Kostenträger Adressaten normativer Steuerung. Dabei gebührt Ihnen gerade im Zeichen neuer Versorgungsformen Spielraum, selbst steuernd aktiv zu werden,

310 Siehe Mayntz/Derlien, Die Organisation der gesetzlichen Krankenversicherung, 1979, S. 6 ff.

311 Projekt im Auftrag des Bundesministeriums für Verkehr, Bau und Stadtentwicklung, siehe unter www.bmvbs.de/beauftragter/Gesellschaft-staerken/Gesundheits-und-Alterversorgun-,1857/Integrier te-Versorgung.htm; ferner unter www.sidiv.de.

312 BGH, Beschl. V. 16.1.2008, KVR 26707, NZS 2008, S. 653 (657).

313 Vgl. a.a.O. gegen eine „kartellrechtliche Bereichsausnahme für Krankenhausfusionen“. 
z.B. durch eine gute ,performance“ bei der Bereitstellung von Gesundheitsleistungen, „,normativ“ durch Vereinbarung besonderer Konditionen, rechtlich-organisatorisch durch Einschaltung von Betreibergesellschaften, im Rahmen der Binnenkooperation oder durch Steuerung der Patientenströme bei Ausweitung der ambulanten Tätigkeit. Leistungserbringer sind „Steuerungsadressaten auf Mikro-Ebene“.314 Managementgesellschaften, die selbst keine Leistungserbringer sind, steuern ggf. ihrerseits die Akteure.

\subsubsection{Adressaten (II) - (Spitzen-)Verbände und korporatistische Steuerung}

Auch Verbände, berufsständische Vertreter und Ausschüsse sind im deutschen Gesundheitswesen intensiv in das Steuerungsgeflecht eingebunden. Diese selbst üben zumeist normativ prägende Steuerungsfunktionen aus, vereinbaren z.B. normsetzende Verträge, insbesondere im Recht der (kollektiven) Vergütungsvereinbarung. Beteiligt an einem Normsetzungsvertrag zur Vergütung der vor- und nachstationären Behandlung (vgl. § 115a Abs. 3 SGB V) bspw. sind die Landesverbände der Krankenkassen, die Ersatzkassen, der Landesausschuß des Verbandes der privaten Krankenversicherung, Landeskrankenhausgesellschaft bzw. Vereinigungen der Krankenhausträger im Land und Kassenärztliche Vereinigungen. Auch die Vereinbarung zum Fallpauschalensystem (vgl. § 17 Abs. 2 KHG) ist in ähnlicher Weise korporatistisch vereinbart, so wie den Verbänden durch den Gesetzgeber aufgegeben, und dies zeigt, daß er delegiert und sich (existente oder kreierte) korporatistische Strukturen zunutze macht. Der z.T. beschriebene „Paradigmenwechsel“315 von der (kollektivistischen) Korporation zur (individuellen) Kooperation ist daher jedenfalls nicht durchgängig vorzufinden. Funktional sind Verbände und Spitzenorganisationen Träger delegierter Verantwortung.

\subsubsection{Steuerungsansätze und -instrumente}

Der Gesetzgeber kann berechtigen oder verpflichten, Direktiven formulieren, Anreize schaffen, begünstigen und sanktionieren, Vertragsschluß- und Gestaltungsfreiheit einzuräumen, Organisationsformen vorschreiben oder zur Wahl stellen. All dies erfolgt in den Grundkoordinaten Wettbewerb und Regulierung sowie Korporatismus und Einzelvertragsprinzip, die in relativer Nähe zueinander stehen und einander nicht ausschließen. ${ }^{316}$ Das Kooperationsprinzip kann sowohl korporatistisch als auch wettbewerbsorientiert ausgeprägt sein. Gerade der Wettbewerb der Vertrags- und Gesellschaftsrechte vermag seinerseits indirekt zu steuern, indem sich dort neue, individuelle Steuerungskonzepte entwickeln sowie konkurrieren und erfolgreiche Strukturen Modellcharakter entfalten und ggf. später gar vorgeschrieben werden.

314 Gerlinger, Zwischen Korporatismus und Wettbewerb: Gesundheitspolitische Steuerung im Wandel, Veröffentlichungen der Arbeitsgruppe Public Health, Wissenschaftszentrum Berlin für Sozialforschung (2002), S. 7; vgl. auch S. 21.

315 Gerlinger, a.a.O. S. 7.

316 Vgl. auch nachfolgend I.2.3.2. 


\subsubsection{Steuerungsaufwand und -anreize; Datenrelevanz}

Differenzierte Systeme sind mitunter selbstreferentiell, v.a. aber in hohem Maße interdependent. In der GKV, in der Wettbewerb mittlerweile untrennbar mit dem morbiditätsorientierten RSA im Gesundheitsfonds verbunden ist, sind Steuerungsfragen kaum mehr isoliert zu betrachten. Der mit der Normierung und Verwaltung verbundene Aufwand ist entsprechend. Der zielinduzierten folgt möglicherweise die folgeninduzierte Steuerung, vor allem, soweit Marktteilnehmer der Versuchung erliegen, „die Gesetze“ reformintendierter Steuerung auszuhebeln. In einem System, das jenseits gesonderter Steuerungsmechanismen zur Förderung von Prävention in differenzierter Form an der datengestützten Abbildung von Morbidität anknüpft, um Kostenbedarf und wettbewerbsneutrale Ausgangspositionen ebenso zielgenau darzustellen wie Vergütung zu organisieren, ist darauf zu achten, (rechtlich unzulässigen) Versuchen, den „input“ zu eigenen Gunsten zu manipulieren, „nachsteuernd“ entgegenzuwirken.

In der GKV erfolgen sowohl die Krankenhausvergütungen durch die Krankenkassen als auch die finanziellen Zuweisungen an die Krankenkassen selbst v.a. morbiditätsbzw. fallorientiert. Diagnosen von Krankheiten und diagnosegestützten FallZuordnungen kommt damit neben der medizinischen Bedeutung im engeren und des Medizinmanagements (Behandlungsleitpfade, Prozessoptimierung etc.) in besonderer Weise wettbewerbs- und finanzierungsrelevante systemische Bedeutung zu. (Risikoadjustierte) Zuweisungen aus dem Gesundheitsfonds konturieren den Maßstab effizienten Handelns der Kassen, denn je nachdem, ob sie mit diesen auskommen, müssen sie einen Zusatzbeitrag erheben bzw. können eine Prämie ausschütten, vgl. § 242 Abs. 1 und 2 SGB V. Veränderungen im DRG-Katalog haben als Neuberwertung der Leistungsstruktur Einfluss auf den Case-Mix, ${ }^{317}$ d.h. die Summe aller Kostengewichte aller Behandlungsfälle eines Krankenhauses, und die richtige aufwandsorientierte Einschätzung entscheidet auch hier über effizientes Wirtschaften.

Aufgrund dieser Bedeutung eröffnet sich ein komplexes Feld der Regulierung des Umgangs mit Diagnosedaten, sei es im Hinblick auf deren Erhebung, Codierrichtlinien, Datenaufbereitung, Datenmeldung und -übertragung, Datenprüfung etc. Vor allem das erlös- oder zuweisungserhebliche Kodierverhalten rückt in den Vordergrund. „Exaktes Kodieren" ist daher aus Sicht der Marktteilnehmer entscheidend. Steuerungsprobleme aber treten vor allem unter zwei Gesichtspunkten auf. Erstens, wenn bisher „ungenaues“ Kodieren dem fortan exakten Kodieren in einer Weise weicht, die - würde nicht steuernd begrenzt - als ,unechte Mehrerlöse“ den effizienten Ressourceneinsatz unter Druck brächten, und zweitens, wenn manipuliert wird. Demgemäss tritt neben die präventive die korrigierende Steuerung. Insbesondere muss Manipulationsverhalten identifiziert und unterbunden werden, speziell wenn es Marktteilnehmer unternehmen, die Erhebung, Aggregation oder Übermittlung von Morbiditätsdaten unter ökonomischen Ge-

317 S. Wasem/Rotter/Focke/Igl, in. Klauber/Robra/Schellschmidt, Krankenhaus-Report 2007 (2008), S. 49 (50f.). 
sichtspunkten in rechtlich unzulässiger Weise zu beeinflussen. Soweit derartige „Fehlanreize“ auszumachen sind, ist „gegen-“ oder auch „,nachzusteuern“, sei es aufsichtsrechtlich oder ggf. normativ (korrigierend).

\subsubsection{Akzente politischer Diskussion um die Krankenhausversorgung}

Unter dem Blickwinkel normativer Steuerung ist die Diskussion über die Zukunft der Krankenhausversorgung wesentlich durch das Erstarken des Individualvertragsprinzips auf den unter 2.1.3 bis 2.1.8 skizzierten Wettbewerbsfeldern geprägt. Aus Sicht der Krankenhäuser geht es darum, Partner in allen relevanten Wettbewerbsbeziehungen zu gewinnen, seien es Patienten, Kostenträger, Management-Partner oder Leistungserbringer, mit deren Hilfe die Angebotsstruktur im Rahmen des Versorgungsmanagements komplettiert oder gesteigert werden kann. Nur ausnahmsweise kommen insoweit auch andere Krankenhäuser z.B. als Verbundpartner in Betracht, was wettbewerbsrechtlich stets kritisch zu beäugen ist.

Der Fokus der politischen Diskussion liegt dabei neben der Schaffung der „krankenhaussektoralen" finanzierungs- und der wettbewerbsrechtlichen Rahmenbedingungen in der Bereitstellung spezifischer Selektivvertragstypen, um Spielräume für Struktur-, Qualitäts- und Kostenoptimierung zu schaffen. Dabei wird zugleich geregelt, wer unter welchen Bedingungen am Selektivvertragswettbewerb teilnimmt. Einige Akzente wurden das GKV-WSG gesetzt (s.o.).

Weitergehende, die Diskussion durchaus prägende Erwartungen, der Gesetzgeber würde einen „echten“ Einstieg in ein Selektivvertragssystem derart ermöglichen, daß nicht nur die transsektoral relevante Versorgung, sondern - § 73c SGB V vergleichbar auch eine „besondere stationäre Versorgung“ (zusammen mit einer Regelung zur Vergütungsbereinigung) geschaffen werden könnte, 318 wurden indes ebenso enttäuscht wie die unterbliebene Einführung der monistischen Finanzierung. 319

Beide Themenkomplexe bleiben danach v.a. in der Wissenschaft auf der Agenda. Da für solchermaßen grundlegende Veränderungen im Zusammenhang mit dem Erlass des KHRG (2009) kein Konsens zu erzielen war, kaprizierte man sich hier auf den (v.a. technischen) Anpassungsbedarf betreffend die Angleichung der Krankenhausbudgets an landesweit einheitliche Preise. Gleichzeitig aber hielt der Gesetzgeber die Weiterentwicklung auch der Investitionsfinanzierung für geboten. Für die in den Krankenhausplan eines Landes aufgenommenen Krankenhäuser soll ab dem 1.1.2012 eine Investitionsfinanzierung durch leistungsorientierte Investitionspauschalen ermöglicht werden, wobei die Länder eigenständig zwischen dieser Form und der weiterhin möglichen Einzelförderung entscheiden können sollen. Von daher steht zu erwarten, das im Bereich der Finanzierung das Thema Monistik zunächst in den Hintergrund gerät. Stattdessen wird (vgl. § $10 \mathrm{KHG)} \mathrm{über} \mathrm{die} \mathrm{bis} \mathrm{zum} \mathrm{31.12.2009} \mathrm{zu} \mathrm{präsentierenden} \mathrm{„Grundsätze} \mathrm{und}$

318 Vgl. die Stellungnahme zum KHRG von Ebsen, Drs. 16(14)90447(39).

319 Vgl. oben nach I. 
Kriterien für die Ermittlung eines Investitionsfallwertes auf Landesebene“ sowie über „grundlegende konzeptionelle Vorgaben“ zu „Grundstrukturen für leistungs- und sachgerechte Investitionsbewertungsrelationen“, „Differenzierungsgrade“ sowie die „differenzierte Entwicklung und Kalkulation bundeseinheitlicher Investitionsbewertungsrelationen durch das DRG-Institut" diskutiert werden. 320

Ungeachtet der Grundsatzdiskussion um Rahmenbedingungen steht die Bewältigung praktischer Herausforderungen im Fokus. Der zunehmende Wettbewerb der Krankenhäuser untereinander, insbesondere im Zeichen der Einführung und Fortentwicklung der DRG, hat zu einem Fusions-, Privatisierungs- und Verdrängungswettbewerb geführt, in dessen Folge auch planerisch-regulatorische Überlegungen wieder in den Vordergrund gerückt werden, wie die Versorgung in ländlichen Räumen sicherzustellen ist. Dies führt zu Zielkonflikten zwischen Sicherstellung und wirtschaftlicher Leistungserbringung. 321

Ein ähnliches Phänomen zeigt sich bei MVZ-Gründungen in Ballungsräumen. Zudem stießen Krankenhäuser, z.B. bei MVZ-Gründungen oder bei Verhandlungen über die IV, auf den Widerstand niedergelassener Ärzte, denen oftmals Managementkapazitäten bzw. finanziell tragbare Beratungsmöglichkeiten fehlen. In einem ersten Schritt führte dies zur Verabschiedung des VÄndG, mit dem strukturelle Einengungen der Tätigkeit der Ärzteschaft beseitigt wurden, z.B. mit besseren Anstellungsmöglichkeiten von Ärzten, der Zulassung örtlicher und überörtlicher Berufsausübungsgemeinschaften zwischen den zugelassenen Leistungserbringern oder der Möglichkeit, gleichzeitig als angestellte Ärzte in Krankenhäusern zu arbeiten.

Schließlich steht die Verbesserung der Qualität im Vordergrund, sei es im Rahmen der ambulaten Behandlung in Krankenhäusern, der Beteiligung an DMP, im Zusammenhang mit der - nunmehr auch sozialrechtlichen - Fortbildungspflicht der Ärzte sowie der evidenzbasierten Medizin; die Qualitätsregulierungen haben maßgeblichen Einfluß auf die Angebotsseite. 322

In diesem Sinne befindet sich der „Krankenhausmarkt [weiter] im Umbruch“, 323 v.a. in den Bereichen Privatisierung, Kooperation und Fusion, Investition, Urbanisierung, Qualitäts- und Angebotsbildung.

320 S. näher unter I.3.4.1. und I.3.4.2.

321 Neubauer/Beivers/Minartz, Marktwandel und Sicherstellung der regionalen Krankenhausversorgung, in: Klauber/Robra/Schellschmidt, Krankenhaus-Report 2006 (2007), S. 65-85.

322 Geraedts, Auswirkungen von Qualitätsregulierungen auf das Angebot von Krankenhausleistungen, in: Klauber/Robra/Schellschmidt, Krankenhaus-Report 2006 (2007), S. 187-203. S. zur sozialrechtlichen Fortbildungs- und Fortbildungsnachweispflicht der Ärzte, Ehlers/Sichert, pharmind. 7/2004, S. 824-826.

323 Gleichnamiger Titel des Krankenhaus-Reports 2006 (2007), a.a.O. 


\section{Die Krankenhausversorgung}

\subsection{Der „,Krankenhaussektor”}

Die Bestimmung des „Sektors“ Krankenhaus hängt maßgeblich vom Krankenhausbegriff und funktionalen Anknüpfungen ab. Steuerungseffekte lassen sich statistisch nur untermauern, wenn sie „sektorenspezifisch“ zugeordnet werden können. Dies korrespondiert mit der „Aufgabenzuordnung im Gesundheitssystem“, etwa in Bezug auf Planung und Finanzierung, sowie mit der „Einordnung in das sozialversicherungsrechtliche Beziehungssystem von Krankenkassen und Leistungserbringern“.324 Gerade letztere ist im Bereich wettbewerbsorientierter Selektivvertragsbeziehungen bedeutsam. Vorsorge und Rehabilitationseinrichtungen werden in Übereinstimmung mit $\S 107$ Abs. 2 SGB V nicht als Krankenhäuser angesehen. Dieser für die Vertrags- und Wettbewerbsbeziehungen wichtige Befund steht im Einklang mit dem Zweck einer öffentlichen Investitionsfinanzierung.

Vorsorge- oder Rehabilitationseinrichtungen nach $\S 107$ Abs. 2 SGB V werden gem. $\S 5$ Abs. 1 Nr. 7 KHG nicht gefördert, obwohl der weite Krankenhausbegriff des $\S 2 \mathrm{Nr}$. 1 KHG diese Einrichtungen einschließt. ${ }^{325}$ Ungeachtet dessen steht es den Ländern gem. $§ 5$ Abs. 2 KHG frei, nach Landesrecht eine entsprechende Förderung zu bestimmen. Für die Untersuchung maßgeblich ist mithin der sozialrechtliche Krankenhausbegriff gem. § 107 Abs. 1 SGB V.

Beim Begriff der „Praxisklinik“ ist zu differenzieren. Der sozialrechtliche Begriff i.S.d. $\S 115$ Abs. 2 S. 1 Nr. 1 SGB V benennt ,Einrichtungen, in denen die Versicherten durch Zusammenarbeit mehrerer Vertragsärzte ambulant und stationär versorgt werden“. Diese Praxiskliniken bedürfen im Hinblick auf die Erbringung von Krankenhausleistungen der Zulassung nach § 108 f. SGB V (s. v.a. § 108 Nr. 3 i.V.m. § 109 SGB V). 326 Nur diesen Begriff kann der mit dem KHRG ergänzte $\S 140 \mathrm{~b}$ Abs. 1 Nr. 7 SGB V im Sinn haben, dem zufolge Praxiskliniken fortan als Vertragspartner i.R.d. integrierten Versorgung auftreten können. Diese „Praxiskliniken“ sind dem „Sektor Krankenhausversorgung" zuzurechnen. Hingegen umfasst der berufsrechtliche Begriff eine ankündigungsfähige Bezeichnung zur öffentlichkeitsorientierten Information über die besondere organisatorische Einrichtung der Praxis eines niedergelassenen Arztes. Dort erfolgt keine stationäre Versorgung, auch wenn „Praxiskliniken“ i.R.d. grundsätzlich ambulanten Versorgung bei Bedarf ärztliche bzw. pflegerische Betreuung über Nacht ebenso zu gewährleisten haben wie hinreichende Vorkehrungen für eine Notfallversorgung. 327

324 Quaas, in: Wenz (Hrsg.), Handbuch des Fachanwalts Medizinrecht (2007), Kap. 12, S. 1118 f.

325 Siehe auch Quaas, a.a.O.

326 Vgl. auch Köhler-Hohmann (2007), juris-PK SGB V, § 115 Rn. 33.

327 In den neueren (Landes-)Berufsordnungen kommt der Terminus „Praxisklinik“, vormals verankert in den jeweiligen Teilen D (Ergänzende Bestimmungen zu den einzelnen ärztlichen Berufspflichten, 


\subsection{Organisationsstruktur des Krankenhaussektors}

\subsubsection{Determinanten}

Wesentliche Charakteristika des Sektors „Krankenhausbehandlung“ haben sich bereits im Zusammenhang mit der Bedeutung der Versorgungssektoren, Behandlungsund Vergütungsformen sowie den Kostendaten ergeben. ${ }^{328}$ Was das Krankenhauswesen in rechtstatsächlicher und steuerungspolitischer Hinsicht ferner auszeichnet, lässt sich an seinen Organisations-, Träger- und Betriebsstrukturen sowie an den leistungsspezifischen Kennzahlen ermessen.

\subsubsection{Zahl, Typologie und Organisation der Krankenhäuser}

Ende 2007 existierten in der Bundesrepublik Erhebungen des Statistischen Bundesamtes (StBA) zufolge 2.087 Krankenhäuser und damit trotz gestiegener Fallzahl (+ 345.000) 17 weniger als im Vorjahr und rund 300 weniger als 15 Jahre zuvor. ${ }^{329}$ Die Zahl der gesondert erfassten Vorsorge- und Rehabilitationseinrichtungen, die hier nicht weiter verfolgt werden, lag im Jahr 2007 bei 1.239. 330

\subsubsection{Trägerschaft}

Das Krankenhauswesen zeichnet sich durch eine pluralistische Trägerstruktur aus. In $\S 1$ Abs. 2 KHG kommt die gesetzliche Anerkennung dieser „Vielfalt der Krankenhausträger" zum Ausdruck.

Nach Art der Trägerschaft sind 677 der im Jahre 2007 registrierten 2.087 Krankenhäuser öffentliche, 790 freigemeinnützige und 620 private Einrichtungen. ${ }^{331}$ Von den öffentlichen Krankenhäusern werden 380 in privatrechtlicher und 297 in öffentlichrechtlicher Form - davon wiederum 161 rechtlich unselbständig und 136 rechtlich selbständig - betrieben. Im Jahre 2002 noch existierten von 817 öffentlichen Häusern 231 in privatrechtlicher und 586 in öffentlich-rechtlicher Form, von denen wiederum 465 noch rechtlich unselbständig waren.

Öffentliche Krankenhäuser werden von Gebietskörperschaften oder durch deren $\mathrm{Zu}$ sammenschlüsse (z.B. Arbeitsgemeinschaften oder Zweckverbänden) sowie von Sozialversicherungsträgern betrieben oder unterhalten. Werden sie nicht als rechtlich unselb-

dort: Kommunikation bzw. Formen der Zusammenarbeit) nicht mehr vor. Ebenso wenig werden die „Anforderungen“ an eine Praxisklinik weiterhin normiert. Stattdessen wird im Wege eines Generaltatbestandes im Rahmen der Berufsregeln zur Kommunikation im Hauptteil (meist § 27) auch die Ankündigung „organisatorischer Hinweise“ gestattet, unter die auch die „Praxisklinik“ fällt.

328 Vorstehend I.2.1. und I.2.2.

329 StBA, Grunddaten der Krankenhäuser 2007 (2008), Allgemeiner Überblick 2007 sowie Ziff. 1.1.

330 Siehe StBA unter www.destatis.de (Gesundheitswesen; Krankenhäuser und Vorsorge- oder Rehabilitationseinrichtungen; Vorsorge- oder Rehabilitationseinrichtungen; Einrichtungen, Betten und Patientenbewegung).

331 StBA, Grunddaten der Krankenhäuser 2007 (2008), Allgemeiner Überblick 2007, Ziff. 1.4. 
ständige Einrichtungen (Eigenbetriebe, Regiebetriebe), sondern in rechtlich selbständiger Form (z.B. als $\mathrm{GmbH}$ ) betrieben, handelt es sich um Krankenhäuser in öffentlicher Trägerschaft dann, wenn die Träger ,unmittelbar oder mittelbar mit mehr als 50 vom Hundert des Nennkapitals oder des Stimmrechts beteiligt sind.“332 Rechtlich selbständig sind auch die in öffentlich-rechtlicher Trägerschaft von den Kommunen als (gemeinsame) ${ }^{333}$ Kommunalunternehmen betriebenen Krankenhäuser. Soweit öffentliche Krankenhäuser zulässigerweise in der Form des Privatrechts betrieben werden, handelt es sich meist um eine (formelle) Organisationsprivatisierung. Auch eine funktionelle und - mit Einschränkungen - eine materielle Privatisierung, d.h. die vollständige Privatisierung in Form der Aufgabenprivatisierung ohne den Erhalt substantieller Einwirkungsmöglichkeiten durch einen öffentlichen Träger, die mit einem Wechsel der Trägerschaft in die Hände von Privaten verbunden ist, sind in der Regel zulässig. Öffentliche Krankenhäuser sind ihrer Zwecksetzung gemäß bedarfswirtschaftlich ausgerichtet. $334 \mathrm{Im}$ Krankenhauswesen sind speziell die Kommunen der Daseinsvorsorge in Form der subsidiären Vorhaltung eigener Krankenhäuser zur Versorgung der Bevölkerung mit leistungsfähigen Krankenhäusern verpflichtet. ${ }^{335}$

Private Einrichtungen bedürfen als gewerbliche Unternehmen (Privatkrankenanstalten, Privatentbindungsanstalten, Privatnervenkliniken) nach $\S 30$ GewO einer Konzession der zuständigen Behörde. Sie arbeiten eigenwirtschaftlich und streben nach Gewinn. ${ }^{336}$ Nach $\S 1$ Abs. 2 S. 2 KHG ist bei der Durchführung des KHG nach Maßgabe des Landesrechts die wirtschaftliche Sicherung privater Krankenhäuser zu gewährleisten. Private Krankenhäuser werden überwiegend in den Rechtsformen $\mathrm{GmbH}$ und der Aktiengesellschaft betrieben. Ferner kommen die ärztliche Privatklinik, der eingetragene Verein ( $\S 21 \mathrm{ff}$., 55 ff. BGB), die Stiftung privaten Rechts ( $\S 80-88$ BGB) sowie die Genossenschaft in Betracht. 337

Nach $\S 1$ Abs. 2 S. 2 KHG ist auch die Sicherung der freigemeinnützigen Einrichtungen zu gewährleisten. Bei diesen handelt es sich um Einrichtungen, „die von Kirchen, von Verbänden der Freien Wohlfahrtspflege oder von Stiftungen unterhalten werden, und die im Sinne der Gemeinnützigkeitsregelungen der Abgabenordnung ohne Absicht der Gewinnerzielung arbeiten."338 Das Vermögen ist an einen bestimmen Zweck gebunden, und die wirtschaftliche Zielsetzung folgt vorwiegend der Bedarfsde-

\section{Grunddaten (2007), Erläuterungen zu Grunddaten.}

333 Vgl. Art. 25 BayKrG; zum gemeinsamen Kommunalunternehmen nach bayerischem Landesrecht Becker, in: ders./Heckmann/Kempen/Manssen, Öffentliches Recht in Bayern, 3. Aufl. (2005), Rn. 638 ff.

334 Freigemeinnützige Krankenhausträger im System staatlicher Krankenhausfinanzierung (1986).

335 Dazu BVerfGE 83, 363, 378; Tettinger, in: von Mangoldt/Klein/Starck, GG, Kommentar, Bd. $2,5$. Aufl. (2005), Art. 28 Abs. 2 Rn. 213.

336 Hamann, Ziele und Strategien der Krankenhausfusion (2000), S. 26.

337 Hamann, a.a.O., S. 28.

338 Hamann, a.a.O., S. 25 m.w.N. 
ckung. 339 Wie die Privaten können sich auch freigemeinnützige Träger der Rechtsformen des Zivilrechts bedienen. Die Form der Stiftung taucht hier häufiger auf.

\subsubsection{Krankenhaustypen und -arten}

Nach Krankenhaustypen unterscheidet die Krankenhausstatistik allgemeine Krankenhäuser, „die über Betten in vollstationären Fachabteilungen verfügen“, 340 sonstige Krankenhäuser, d.h. solche „mit ausschließlich psychiatrischen, psychotherapeutischen und/oder neurologischen Betten und reine Tages- oder Nachtkliniken" sowie Bundeswehrkrankenhäuser. 1.791 der insgesamt 2.087 Krankenhäuser sind Allgemeine, 296 sonstige Krankenhäuser. 341

Diese Einteilung ist nicht deckungsgleich mit einer an der Aufgabenstellung ausgerichteten Unterscheidung nach Krankenhausarten, nach Allgemein-, Fach- und Sonderkrankenhäusern, 342 wenngleich diese ursprünglich auf die amtliche Krankenhausstatistik zurückgeht. ${ }^{343}$ Allgemeinkrankenhäuser nehmen Kranke ohne Rücksicht auf Alter und Art der Erkrankung auf. In Fachkrankenhäusern werden Kranke mit bestimmten Krankheitsarten oder Kranke bestimmter Altersstufen aufgenommen; sie sind nach der Fachrichtung meist weiter zu differenzieren. Sonderkrankenhäuser ermöglichen besondere Formen der Unterbringung (psychiatrische Krankenhäuser), dienen der Behandlung bestimmter Personengruppen oder sind auf die Anwendung bestimmter Behandlungsverfahren spezialisiert (Tuberkulosekrankenhäuser). Je nach Ausrichtung können sie Allgemein- oder Fachkrankenhäuser sein. Die an der Verweildauer orientierte Unterscheidung zwischen Akutkrankenhäusern (Verweildauer bis zu vier Wochen) und Langzeitkrankenhäusern hat im Zeichen jüngerer Reformen der Krankenhaus- und Vergütungsstrukturen an Bedeutung verloren. Nach einer Häufung von schwerkranken Patienten oder aus Gründen der Versorgungsstruktur und Vergütung Besondere Einrichtungen (gem. § 17b Abs. $1 \mathrm{~S}$. $15 \mathrm{KHG)} \mathrm{sollen} \mathrm{hier} \mathrm{ebenso} \mathrm{vernachlässigt} \mathrm{werden} \mathrm{wie}$ eine Gliederung nach Versorgungsstufen. Im Gegensatz zu Anstaltskrankenhäusern werden in Belegkrankenhäusern schließlich Leistungen von freiberuflich tätigen, nicht am Krankenhaus angestellten Ärzten, d.h. Belegärzten mit Liquidationsberechtigung erbracht, vgl. § 18 KHEntgG.

339 Faltin, Freigemeinnützige Krankenhausträger im System staatlicher Krankenhausfinanzierung (1986), S. 14.

340 „Wobei die Betten nicht ausschließlich für psychiatrische und/oder neurologische Patientinnen oder Patienten vorgehalten werden".

341 StBA, Grunddaten der Krankenhäuser 2005 (2006), Ziff.2.1.1.

342 Dazu und den folgenden Kategorien Schmidt (2004-2009), in: Peters, Handbuch der Krankenversicherung, SGB V, § $39 \mathrm{Rn} .71 \mathrm{ff}$.

343 Schmidt (2009), a.a.O., § 39 Rn. 71. Hinzu kommt ein sachlich-terminologischer Wechsel in der Abgrenzung der sonstigen Krankenhäuser seit dem Jahre 2002, vgl. die Grunddaten (2003), Erläuterungen. 


\subsubsection{Unterscheidung nach Art der Zulassung}

Nach Art ihrer Zulassung unterscheidet § 108 SGV Hochschulkliniken im Sinne des Hochschulbaufinanzierungsgesetztes (Nr. 1), Krankenhäuser, die in den Krankenhausplan eines Landes aufgenommen sind (Plankrankenhäuser, Nr. 2) sowie Krankenhäuser, die einen Versorgungsvertrag mit den Landesverbänden der Krankenkassen und den Verbänden der Ersatzkassen abgeschlossen haben (Nr. 3). Darüber hinaus weisen die Grunddaten des Statistischen Bundesamtes (StBA) Krankenhäuser ohne Versorgungsvertrag aus, die in keine der vorgenannten Kategorien fallen. ${ }^{344}$

Eine materielle Privatisierung der Universitätsklinika ist ausgeschlossen. ${ }^{345}$ Im übrigen kann der öffentliche Träger ,zwischen der unselbständigen und selbständigen Anstalt des öffentlichen Rechts, der Körperschaft des öffentlichen Rechts sowie privaten Rechtsformen wählen.“"346

Von den 2005 registrierten 1846 Allgemeinen Krankenhäusern waren ihrer Zulassungsart nach 34 Hochschulkliniken, 1468 Plankrankenhäuser und 101 Vertragskrankenhäuser; hinzu kommen 143 Krankenhäuser ohne Versorgungsvertrag. Obwohl die Hochschulkliniken nur $1,8 \%$ der Akutkliniken ${ }^{347}$ ausmachen, halten sie einen Anteil von 9,1\% der Akutbetten und 9,5\% der Fälle. ${ }^{348}$ Für die Plankrankenhäuser sind 86,3\% Akutklinikanteil bei $89 \%$ der Betten und Fälle, für die Vertragskrankenhäuser 1,4\% Betten- und 1,2\% Fallzahlanteil bei einem Akutklinikanteil von 4,6\% zu verzeichnen.

\subsubsection{Aufgestellte Betten und Patientenbewegung}

Die Gesamtzahl der in den Krankenhäusern aufgestellten Betten, d.h. der „betriebsbereit aufgestellten Betten des Krankenhauses, die zur vollstationären Behandlung von Patienten und Patientinnen bestimmt" und nach dem Jahresdurchschnittswert ermittelt worden sind, ${ }^{349}$ lag im Jahre 2007 bei 506.954 , d.h. 243 pro Einrichtung. ${ }^{350}$ Gegenüber 1995 (2.325 Häuser mit durchschnittlich 262 Betten) bedeutet dies einen Rückgang um mehr als 100.000 Betten. Die öffentlichen Häuser verfügten 2007 über 250.345 Betten,

344 Vgl. zur Definition (in Abweichung zu den Vorjahren) und Konsequenzen bei der Vergleichbarkeit und Entwicklung StBA, Grunddaten der Krankenhäuser 2005 (2006), Ziff. 2.1.1. In den Erläuterungen zu einzelnen Erhebungsmerkmalen 2003 waren diese Häuser ungünstigerweise als ,sonstige Krankenhäuser" aus, obwohl es sich hier dem Krankenhaustyp nach um allgemeine und nicht um „sonstige“ Krankenhäuser handelt: vgl. dort Ziff. 2.1.1.

345 Stefan Becker, Das Recht der Hochschulmedizin (2005), S. 155 ff. Zur Rechtsform des Universitätsklinikums ab S.121 ff.

346 A.a.O., S. 169.

347 Prozentuale Auswertung durch Rau (2007), in: O/R/S/W/Z, SGB V, § 108 Rn. 4, für das Jahr 2003. Der hier verwendete Begriff der Akutkliniken entspricht letztlich dem statistikbezogenen Begriff der ,allgemeinen Krankenhäuser“, vgl. vorstehend.

348 Rau, a.a.O.

349 StBA, Grunddaten der Krankenhäuser 2007 (2008), Erläuterungen.

350 A.a.O., Ziff. 1.1. bzw. 1.3. 
die freigemeinnützigen Einrichtungen über 177.632, und die privaten Einrichtungen, die fünf Jahre zuvor gut 30.000 weniger stellten, über 78.977 Betten. ${ }^{351}$

Fallzahl und Bettenauslastungen nehmen seit 2005 (16,5 Mio. bzw. 74,9\%) stetig leicht zu (2007: 17,1 Mio. bzw. 77,2\%). ${ }^{352}$ Betrug die Verweildauer im Jahre 1995 noch 11,4 Tage bei einer Bettenauslastung von 82,1\% (1991: 14 Tage, 84,1\%), so sank sie bis 2007 auf 8,3 Tage; ${ }^{353}$ nimmt man die Krankenhäuser mit ausschließlich psychiatrischen, psychotherapeutischen und/oder neurologischen Betten mit einer Verweildauer von durchschnittlich 25,5 Tagen aus dieser Betrachtung heraus, ergibt sich eine durchschnittliche Verweildauer in allgemeinen Krankenhäusern von erstmals unter 8, nämlich 7,8 Tagen.

\subsection{4. Ärztliches und nichtärztliches Personal}

Rund 1,07 Mio. Menschen waren in den Krankenhäusern insgesamt beschäftigt, 3.000 oder $0,3 \%$ weniger als im Jahr davor. ${ }^{354}$ Umgerechnet in Vollzeitkräfte betrug die maßgebliche Zahl der Beschäftigten gut 792.000 im Jahrsdurchschnitt, davon $126.000(+1,8 \%)$ im ärztlichen und 666.300 (- 0,3\%) im nichtärztlichen Dienst. ${ }^{355}$ Die Personalstruktur veränderte sich damit wie in den Vorjahren wiederum zugunsten des ärztlichen Personals.

\subsection{Historische Entwicklung}

Die Geschichte des Krankenhauswesens ist geprägt vom Funktionswandel des Krankenhauses ${ }^{356}$ und der Zunahme an staatlichem Einfluss, der v.a. in der Krankenhausfinanzierung zum Ausdruck kommt.

\subsubsection{Funktionswandel der Krankenhäuser}

In historischer Perspektive, die bis ins Mittelalter zurückreicht, erwies sich eine der Krankenversorgung dienende Anstalt lediglich als besondere Form der Hospitäler, welche - getreu dem Wortursprung - mehrere Aufgaben gleichzeitig erfüllten. ${ }^{357}$ Im Vordergrund standen Betreuung, Wohnung und Pflege Bedürftiger, d.h. nicht nur (unheilbar) ${ }^{358}$ Kranker, sondern v.a. armer und alter Menschen und Waisen; vermögende Schichten stützten sich auf die Hilfe der Familie und beorderten den Arzt in das eigene

351 A.a.O., Ziff. 1.4.

352 A.a.O., Ziff. 1.1.

353 A.a.O., 2.2.1.

354 A.a.O., allgemeiner Überblick.

355 A.a.O., Ziff. 2.3.1.

356 Faltin, Freigemeinnützige Krankenhausträger im System staatlicher Krankenhausfinanzierung (1986), S. $5 \mathrm{f}$.

357 Faltin, a.a.O., S. 5.

358 Dieser primären Aufgabe dienten Hospitäler vor allem im Mittelalter, s. Faltin, a.a.O., S. 5. 
Haus. ${ }^{359}$ Die Spitäler befanden sich überwiegend in der Trägerschaft der Kirchen und karitativer Einrichtungen. ${ }^{360}$ Hinzu kamen städtische Anstalten in Form von Leprosorien, Pesthäusern und Anstalten für Geisteskranke. ${ }^{361}$ Die (gezielte) Behandlung des Krankheitsfalles rückte erst mit der Fortentwicklung der Medizin als wissenschaftlicher Disziplin und des technischen Forschritts - insbesondere der Einführung der Narkose, Antisepsis und der Asepsis ${ }^{362}$ - in den Mittelpunkt. ${ }^{363}$ Mit ihr sollte eine Abkehr vom Bild des Krankenhauses als Sterbeanstalt erfolgen. ${ }^{364}$ So wurden seit dem 18. Jh. Unterrichts- und Universitätskliniken errichtet, die häufig der Einteilung in medizinische Fächer folgten, bspw. die gegen Ende des 18. Jh. entstandene Medizinische Klinik, die chirurgische Klinik und die Frauenklinik. ${ }^{365}$ Der führenden Stellung der deutschen Medizin in der zweiten Hälfte des 19. Jh. entsprechend gilt der Zeitraum zwischen 1800 und 1920 als Blütezeit der akademischen Krankenhäuser. ${ }^{366}$ Damit bereitete die (Hochschul-)Medizin auch Krankenanstalten in anderer Trägerschaft den Weg. Die fortschrittlichen Krankenhäuser wurden zunehmend durch breite Bevölkerungsschichten in Anspruch genommen, und diese Entwicklung erhielt durch die Einführung der GKV im Jahr 1883 weiter Auftrieb. 367

Parallel dazu kam es zu einer Neujustierung des Verhältnisses von kirchlicher und staatlicher Wohlfahrt. Konzentrierte sich der Staat bis ins 19. Jh. noch auf die gesundheits- und gewerbepolizeiliche Überwachung der kirchlichen Einrichtungen, übernahmen Staat und Kommunen in Folge der Säkularisation (1803) ${ }^{368}$, im Zeichen der kommunalen Allzuständigkeit für örtliche Angelegenheiten und speziell seit Einführung der GKV in gesteigertem Maße Verantwortung für die soziale Sicherung. ${ }^{369}$ Kirchliche und staatliche Wohlfahrt ergänzten einander, auch dann noch, als es in der Weimarer Zeit zur erneuten Ausweitung staatlicher Verantwortung kam. ${ }^{370}$ Die nach dem zweiten

359 Kies, Der Versorgungsauftrag des Plankrankenhauses (1998). S. 19.

360 Siehe auch Knorr/Kraemer, Krankenhausrecht (2005), Einführung, S. VII.

361 Tuschen/Trefz, Krankenhausentgeltgesetz (2004), S. 14.

362 Kies, Der Versorgungsauftrag des Plankrankenhauses (1998). S. 19; Tuschen/Trefz, Krankenhausentgeltgesetz (2004), S. 15.

363 Vgl. Stefan Becker, Das Recht der Hochschulmedizin (2005), S. 73 f. S. auch Knorr/Kraemer, Krankenhausrecht (2005), Einführung, S. VII.

364 Faltin, Freigemeinnützige Krankenhausträger im System staatlicher Krankenhausfinanzierung (1986), S. 5.

365 Stefan Becker, Das Recht der Hochschulmedizin (2005), S. 73 f. Diese Entwicklung wurde durch den Rückgriff auf Gebäude und den Patientenstamm der Armenhospitäler historischer Prägung gestützt (S. 74).

366 Stefan Becker, a.a.O., S. 75 f.

367 Kies, Der Versorgungsauftrag des Plankrankenhauses (1998). S. 19.

368 Tuschen/Trefz, Krankenhausentgeltgesetz (2004), S. 14 f.

369 Faltin, Freigemeinnützige Krankenhausträger im System staatlicher Krankenhausfinanzierung (1986), S. 28.

370 Faltin, a.a.O., S. 28 f. 
Weltkrieg einsetzenden planenden und regulierenden Eingriffe ${ }^{371}$ betrafen insbesondere das Recht der Krankenhausfinanzierung.

Der Begriff „Krankenanstalten“ findet sich noch heute in der Gewerbeordnung, § 30 GewO. Die dort normierte ,konzessionspflichtige Privatkrankenanstalt“372 beschäftigte bereits das Preußische Oberverwaltungsgericht, das den Bestand von Betten für die zu behandelnden Patienten zur begrifflichen Voraussetzung erhob. ${ }^{373}$

\subsubsection{Historische Entwicklung der Krankenhausfinanzierung}

Die Geschichte der Krankenhausfinanzierung markiert den zweiten Strang der Entwicklung des Krankenhauswesens. Sie verdeutlicht den zunehmenden staatlichen Einfluß und umschreibt ein wichtiges Feld für Reformen. Die Entwicklung der gesetzlichen Krankenhausfinanzierung ist Ausdruck der Wirkungsgeschichte zentraler Steuerungsinstrumentarien für das bereits formulierte Anliegen, ${ }^{374}$ eine kostengünstige Krankenhausversorgung sicherzustellen.

Während die Finanzierung der Spitäler historischer Prägung vorwiegend durch die (kirchlichen, karitativen und städtischen ${ }^{375}$ ) Träger sowie durch Spenden erfolgte, ${ }^{376}$ setzt die jüngere ${ }^{377}$ Geschichte der Finanzierung mit der sog. monistischen (aus einer Quelle gespeisten) Krankenhausfinanzierung ursprünglicher Form ein. Sie funktionierte zunächst in Freiheit von staatlicher Einflussnahme auf die Preis- und Mengengestaltung. Seit Einführung der GKV gewährten die Krankenkassen ihren Versicherten „freie Kur und Verpflegung in einem Krankenhaus“. ${ }^{378}$ Die Krankenkassen zahlten Pflegesätze an die Krankenhäuser auf der Grundlage intensiver vertraglicher Beziehungen. ${ }^{379}$ Mit der sog. Preisstopverordnung des Jahres $1936^{380}$, die Preiserhöhungen mit Wirkung auch für die Krankenhauspflegesätze im wesentlichen ${ }^{381}$ untersagte, wurde in die Vertrags-

371 Faltin, a.a.O., S. 29.

372 Von $\S 30$ GewO ebenfalls erfasst sind die Privatentbindungsanstalten sowie die Privatnervenkliniken.

373 Siehe Faltin, Freigemeinnützige Krankenhausträger im System staatlicher Krankenhausfinanzierung (1986), S. 5 unter Verweis auf PrOVGE 1, 284 (286 f.).

374 Siehe oben I.2.2.

375 Tuschen/Trefz, Krankenhausentgeltgesetz (2004), S. 14.

376 Kies, Der Versorgungsauftrag des Plankrankenhauses (1998). S. 19. Hinzu kam die Finanzierung durch Steuermittel der Gemeinden sowie durch private Stiftungen, vgl. Patt, System und Kontrolle der Preisbildung für Krankenhausleistungen (1996) S. 38.

377 S. zur Einteilung in Phasen Hamann, Ziele und Strategien der Krankenhausfusion (2000), S. 41 ff. m.w.N.

378 Zitiert nach Faltin, Freigemeinnützige Krankenhausträger im System staatlicher Krankenhausfinanzierung (1986), S. 5; Patt, System und Kontrolle der Preisbildung für Krankenhausleistungen (1996) S. 39.

379 Tuschen/Trefz, Krankenhausentgeltgesetz (2004), S. 15.

380 RGB1. I, S. 955, Hamann, Ziele und Strategien der Krankenhausfusion (2000), S. 41.

$381 \mathrm{Zu}$ Einzelheiten Patt, System und Kontrolle der Preisbildung für Krankenhausleistungen (1996) S. 40. 
freiheit nachhaltig eingegriffen. Sie setzt einen Markstein zunehmend regulativer bzw. dirigistischer staatlicher Preisintervention. Alsdann wurden eine weitere Preisrechtsverordnung von $1948^{382}$ als Ersatz für die nach dem 2. Weltkrieg aufgehobene alte Preisstopverordnung sowie die Pflegesatzordnung des Bundes des Jahres 1954 zur Deckung der Selbstkosten erlassen. ${ }^{383}$ Der in letzterer normierte Abzug herkömmlicher Zuschüsse zur Bestimmung der Betriebskosten sowie die Einschränkung der Berücksichtigungsfähigkeit spezifischer Kostenarten vermochten steigende Defizite der (weiterhin monistischen) Krankenhausfinanzierung von bis zu 460 Mio. $€$ jährlich gegen Ende der 60er Jahre ${ }^{384}$ allerdings nicht zu verhindern. ${ }^{385}$

Im Zuge dessen und einer 1969 erfolgten Grundgesetzänderung (Einfügung des Art. 74 Abs. 1 Nr. 19a GG) kam es mit dem KHG von 1972 zur Einführung der dualen Krankenhausfinanzierung, der zufolge die Investitionskosten von der öffentlichen Hand (Bund und Länder) und die Betriebskosten von den Krankenkassen bzw. Patienten zu tragen waren. Damit erhielten die Länder, die von den ihnen nach KHG zustehenden Regelungsbefugnissen Gebrauch gemacht haben, ${ }^{386}$ entscheidenden Einfluß über die Investitionsentscheidung. Das weiterhin in Geltung befindliche (mehrfach geänderte und 1991 neu bekannt gemachte) KHG dient der wirtschaftlichen Sicherung der Krankenhäuser. Die Länder stellen als eigene Angelegenheit Krankenhauspläne auf, die einer bedarfsgerechten Versorgung und der Leistungsfähigkeit der Krankenhäuser verpflichtet sind. ${ }^{387}$ Die seitens der Länder in Investitionsförderprogramme aufgenommenen Krankenhäuser können auf Antrag bestimmte Fördermittel erhalten. 388 Mit der Einführung der dualen Krankenhausfinanzierung galt das Kostendeckungsprinzip, sowohl bzgl. der Investitionskosten als auch für das Preisrecht 389 : Die neue Bundespflegesatzverordnung von 1973 (BPflV) normierte tagesgleiche und vollpauschalierte Pflegesätze. 390

Alsdann wurde das duale Finanzierungssystem angesichts massiver Kostensteigerungen u.a. wegen kostenintensiver überlanger Verweilzeiten und Fehlbelegungen bei überhöhter Zahl der Krankenhausbetten modifiziert. Dies geschah mittels des Krankenhaus-Neuordnungsgesetzes (1984) durch eine „schrittweise Rückkehr zur Monistik“ im Wege der Abschaffung der Bundesfinanzhilfen sowie der Einbindung der Krankenhäu-

382 Näher dazu Patt, a.a.O., S. 41 f.

383 Kies, Der Versorgungsauftrag des Plankrankenhauses (1998). S. 19; Hamann, Ziele und Strategien der Krankenhausfusion (2000), S. 42; Eingehend Patt, a.a.O., S. 42 ff.

384 Knorr/Kraemer, Krankenhausrecht (2005), Einführung, S. VIII.

385 Näher Tuschen/Trefz, Krankenhausentgeltgesetz (2004), S. 17.

386 Kies, Der Versorgungsauftrag des Plankrankenhauses (1998). S. 24 f.

387 Vgl. § 6 Abs. 1 KHG. Das Nähere wird durch Landesrecht bestimmt, § 6 Abs. 4 KHG.

388 Vgl. $\S 6$ Abs. 1 und 4, $\S 8$ und 9 KHG.

389 Knorr/Kraemer, Krankenhausrecht (2005), Einführung, S. VIII.

390 Hamann, Ziele und Strategien der Krankenhausfusion (2000), S. 43; näher Patt, System und Kontrolle der Preisbildung für Krankenhausleistungen (1996) S. 45 f., und Tuschen/Trefz, Krankenhausentgeltgesetz (2004), S. 19 ff. 
ser und Krankenkassen in die Krankenhausplanung durch Abschluss von Investitionsverträgen zur Rationalisierung. ${ }^{391} \mathrm{Im}$ Zeitraum der Mischfinanzierung ab 1972 bis 1984 hat der Bund 10,5 der insgesamt 46 Mrd. DM getragen. ${ }^{392}$ Durch eine neue Bundespflegesatzverordnung wurde mit Wirkung zum Jahre 1986 ein flexibles Krankenhausbudget eingeführt, das ,auf der Grundlage der vorauskalkulierten Selbstkosten unter Berücksichtigung der voraussichtlichen Belegung für einen zukünftigen Zeitraum vereinbart" wurde - dies sollte eine gewinnorientierte Ausweitung der Leistungsmengen ebenso wie Verluste durch Minderbelegung abmildern. ${ }^{393}$

Die anhaltende Finanzierungskrise sowie massive Beitragssatzsteigerungen führten 1992 zum Erlaß des Gesundheitsstrukturgesetzes (GSG) ${ }^{394}$. Die Ausgaben der GKV West waren zwischen 1970 und 1991 von 25,2 auf 159,8 Mrd. DM angestiegen. ${ }^{395} \mathrm{Be}-$ trug der durchschnittliche allgemeine Beitragssatz 1970 noch 8,2\%,396 lag er zum 1. Oktober 1992 auf dem Rekordniveau von 13,1\%. 1992 lag das Ausgabenwachstum bei der Krankenhausbehandlung mit 10,1\% doppelt so hoch wie der Anstieg der Grundlöhne. ${ }^{397}$ Das GSG hob das Selbstkostendeckungsprinzip auf, welches i.V.m. dem tagesgleichen Pflegesatz als ,grundlegende(r) Strukturfehler“ galt. ${ }^{398}$ An der dualen Finanzierung wurde zunächst festgehalten ${ }^{399}$ und mit der Koppelung an die beitragspflichtigen Einnahmen der Mitglieder ein festes (,gedeckeltes") Budget für den Zeitraum der Jahre 1993 bis 1995 auf der Basis des Jahres 1992 eingeführt. ${ }^{400}$ Neue, landeseinheitliche Entgeltformen, die an die Leistung und nicht das vorhandene Bett als Maßstab für Einnahmen, Personalkosten und Investitionsförderung anknüpften, sollten der Unwirtschaftlichkeit durch überlange Verweildauer und Fehlbelegungen im Zeichen des Überangebots an Akutbetten entgegenwirken. 401 Die dazu vorgesehenen Fallpauschalen (zunächst rund 40) für bestimmte Behandlungsfälle, Sonderentgelte (rund 160) für bestimmte Eingriffe sowie Abteilungs- und Basispflegesätze zur Abrechnung krankenhausindividueller Budgets fanden Aufnahme in die BPflV 1995, die ab 1996 verpflichtend galt. Das Entgeltsystem wurde differenzierter, jedoch auch als komplizierter und

391 Kies, Der Versorgungsauftrag des Plankrankenhauses (1998), S 27; Hamann, a.a.O., S. 43 f.; Knorr/Kraemer, Krankenhausrecht (2005), Einführung, S. IX.

392 Tuschen/Trefz, Krankenhausentgeltgesetz (2004), S. 19.

393 Hamann, Ziele und Strategien der Krankenhausfusion (2000), S. 44.

394 Eingehend zu den für den Krankenhausbereich relevanten Regelungen des GSG Tuschen/Trefz, Krankenhausentgeltgesetz (2004), S. 27 ff.

395 BT-Drucks. 12/3608, S. 66.

396 A.a.O., S. 67.

397 A.a.O., S. 67.

398 A.a.O., S. 67.

399 Sie sollte gleichwohl ,schrittweise in einem mittel- und längerfristigen Prozeß durch ein monistisches Finanzierungssystem (Finanzierung insgesamt über Pflegesätze) ersetzt werden“, BT-Drucks. 12/3608, S. 70.

400 BT-Drucks. 12/3608, S. 70; Knorr/Kraemer, Krankenhausrecht (2005), Einführung, S. IX; näher Tuschen/Trefz, Krankenhausentgeltgesetz (2004), S. 31, 33 ff.

401 Vgl. BT-Drucks. 12/3608, S. 67. 
aufwendiger eingestuft. ${ }^{402}$ Flankiert wurden diese Maßnahmen durch erstmals in Angriff genommene Bestrebungen zur Verzahnung von ambulanter und stationärer Versorgung unter Einschluß der Großgeräteplanung, die ebenfalls der Kostensenkung dienten. ${ }^{403}$ Dabei ging es vor allem um einen festen Rahmen für die vor- und nachstationäre Behandlung und den Ausbau der Möglichkeit des ambulanten Operierens sowie um die teilstationäre Durchführung von Operationen.

In der Folgezeit wurden die durch das GSG eingeführten Bestimmungen und die BPflV mehrfach geändert. Kostenkontrolle und Beitragssatzstabilität bildeten die maßgeblichen Leitmotive. Auffällig dabei ist, daß von der Deckelung der Budgets - bei Modifikationen im Detail - nicht abgerückt, sondern diese beibehalten und fortgeschrieben wurde, zuletzt durch das GKV-Gesundheitsreformgesetz 2000 bis zum Jahre 2003. 404

Das Fallpauschalengesetz (FPG) vom April 2002 setzte die durch das GKVGesundheits-Reformgesetz 2000 auch in $\S 17 \mathrm{~b}$ KHG verankerte Verpflichtung um, ,ein durchgängiges, leistungsorientiertes und pauschalierendes Vergütungssystem einzuführen“ (DRG-System). ${ }^{405}$ Es sollte ursprünglich in drei Schritten bis zum Jahre 2007 einführt werden; der Zeitraum wurde allerdings bis zum Jahre 2009 verlängert. ${ }^{406}$ Die komplexe, abgestufte Einführung des seit 2004 verpflichtenden 407 Vergütungssystems steht mit neuen Fallpauschalvereinbarungen vor der Herausforderung der Angleichung der für 2003 und 2004 budgetneutral ${ }^{408}$ und krankenhausindividuell ermittelten Preise für die jeweiligen Fallgruppen an den landesweiten Durchschnitt. ${ }^{409}$ Neben der Einführung der DRGs sah der Gesetzentwurf die erneute Hinwendung zur monistischen Finanzierung vor, wie sie bereits im GSG angeklungen war. Dies sollte durch die schrittweise Rückführung der Finanzierung der Länder bis 2008 geschehen. ${ }^{410} \mathrm{Ab}$ diesem Zeitpunkt sollte die Einzelinvestitionsförderung, ab 2003 die Finanzierung der pauschalen Fördermittel über das neue Vergütungssystem der DRG erfolgen. Die Renaissance des Monismus, mit Modifikationen noch von der Beschlußempfehlung des Ausschusses für

402 Patt, System und Kontrolle der Preisbildung für Krankenhausleistungen (1996), S. 50.

403 BT-Drucks. 12/3608, S. 71.

404 Knorr/Kraemer, Krankenhausrecht (2005), Einführung, S. X.

405 Siehe z.B. oben I.2.1.5.1.

406 Knorr/Kraemer, Krankenhausrecht (2005), Einführung, S. XVII f.

$407 \S 17 \mathrm{~b}$ Abs. 6 S. 1 KHG. Die Teilnahme im Jahre 2003 konnte freiwillig erfolgen, $\S 17 \mathrm{~b}$ Abs. 4 und 5 KHG.

$408 \S 17$ b Abs. 4 S. 1, Abs. 6 S. 3 KHG.

$409 \S 17$ b Abs. 6 S. 4 KHG i.V.m. § 4 KHEntgG. S. unter I.3.4.2.

410 BT-Drucks- 14/1245, S. 56. Das Konzept findet sich auch in dem vom BMG(S) dem Institut für Funktionsanalyse im Gesundheitswesen erteilten Gutachten (oben I.2.2.5.) wieder, vgl. unter www.bmgs.bund.de (unter Themenschwerpunkte/Ressortforschung, Kurzberichte Archiv, 16.3.2000, z.B. S. 143 f. 
Gesundheit gestützt, ${ }^{411}$ konnte sich im Zeichen zwischenzeitlich geänderter Bundesratsmehrheiten allerdings nicht durchsetzen. ${ }^{412}$

Das GKV-Modernisierungsgesetz 2003 - erhebt die (im FPG zuvor umfassend reformierte) Krankenhausfinanzierung nicht zum Schwerpunkt, bindet den Krankenhaussektor jedoch in neue, wettbewerbsorientierte Versorgungsformen mit ein. ${ }^{413}$ Unterdessen erfolgte nach den Fallpauschalenvereinbarungen 2005 und 2006 mit der Einigung zwischen der DKG, den Spitzenverbänden der Krankenkassen und dem Verband der PKV am 19. September 2006 über die FPV 2007 bereits zum dritten Male eine Vereinbarung unter den Selbstverwaltungspartnern, ohne daß - wie noch in den Jahren 2003 und 2004 - eine Ersatzvornahme seitens des Ministeriums erforderlich wurde. ${ }^{414}$ Anstelle der Spitzenverbände der Krankenkassen agiert nach entsprechender Änderung durch das GKV-WSG nunmehr der Spitzenverband Bund der Krankenkassen, ${ }^{415}$ Partner der PFV 2009.

\subsection{Rechtlicher Rahmen}

\subsubsection{Allgemeines}

„Das Krankenhausrecht” hat sich - gegenstandsspezifisch - zunehmend als eigene Materie herausgebildet. Es ist Teil des Medizinrechts, ${ }^{416}$ das ebenfalls eine Querschnittsmaterie bildet. Tragende Säulen, die spezifisch an die Einrichtung „Krankenhaus“" anknüpfen, sind das Recht der Krankenhausfinanzierung, der Krankenhausplanung sowie das Recht der Inanspruchnahme der Leistung und der Leistungserbringung. Darüber hinaus sind in Anknüpfung an die organisationsrechtliche Seite v.a. das Gesellschaftsrecht und das kommunale Wirtschaftsrecht, mit Blick auf die Beschäftigen das Recht der Heilberufe, das (öffentliche) Dienst- und Arbeitsrecht sowie das Arzthaftungsrecht von Bedeutung. Wesentliche Bereiche des Krankenhausrechts sind Bundesrecht und fußen im Bereich der konkurrierenden Gesetzgebung (vgl. Art. 72 GG) auf der Zuständigkeit des Bundes gem. Art. 74 Abs. 1 Nr. 12 GG (Sozialversicherung), Art. 74 Abs.1 Nr. 19 (Zulassung zu ärztlichen und anderen Heilberufen und zum Heilgewerbe) und Art. 74 Abs. 1 Nr. 19a GG (wirtschaftliche Sicherung der Krankenhäuser und Regelung der Krankenhauspflegesätze). Das Berufsrecht der Heilberufe bzw. des Gesundheitsdienstes hingegen obliegt weitgehend den Ländern. Diese sind es weiter, die zur Erfüllung der in § $1 \mathrm{KHG}$ genannten Ziele nach $\S \S 6,8$ ff. KHG Krankenhauspläne

411 BT-Drucks. 14/1977, S. $110 \mathrm{f}$.

412 Siehe Tuschen/Trefz, Krankenhausentgeltgesetz (2004), S. 44.

413 S.o. 2.1.3.

414 Steiner/Bussmann/Koerdt, „Vereinbarung zum Fallpauschalensystem für Krankenhäuser (FPV 2007) - Abrechnung nach der PFV 2007, Redaktionsbeilage/FPV 2007, in das Krankenhaus 1/2007.

415 Siehe Art. 18 Nr. 4 b GKV-WSG (BGB1. I 2007, S. 445 f.) mWv. 1. Juli 2008, Art. 46 Abs. 9 (a.a.O., S. 472).

416 Quaas, in: Wenzel (Hrsg.), Handbuch des Fachanwalts Medizinrecht (2007), Kap. 12, S. 1115. 
und Investitionsprogramme aufstellen, deren Grundsätze und Verfahren in den Krankenhausgesetzen der Länder normiert sind. ${ }^{417}$

\subsubsection{Recht der Krankenhausfinanzierung}

\subsubsection{Grundzüge}

Zum Recht der Krankenhausfinanzierung gehören in erster Linie das Krankenhausfinanzierungsgesetz (KHG), welches der wirtschaftlichen Sicherung der Krankenhäuser sowie der Regelung der Krankenhauspflegesätze dient, sowie das Krankenhausentgeltgesetz (KHEntgG), das die Vergütung der Krankenhäuser regelt, die das DRG-System anwenden. ${ }^{418}$

Das KHG bildet die Grundlage der Krankenhausfinanzierung. Es enthält grundsätzliche Vorgaben auch zur Vergütung für die Vereinbarung der Pflegesätze für die vor- und nachstationäre Behandlung gem. § 115a SGB V (§ 17 Abs. $1 \mathrm{KHG)} \mathrm{sowie} \mathrm{die} \mathrm{Einfüh-}$ rung des pauschalierenden Entgeltsystems ( $§ 17 \mathrm{~b}$ KHG). Mit Blick auf $\S 17 \mathrm{~b}$ KHG sowie die allgemeinen Vorgaben für die Pflegesatzfähigkeit und für die Budget- bzw. Pflegesatzverhandlungen überspannt das KHG die Finanzierung insgesamt; $\S 1$ KHEntgG bestimmt daher, daß die vollstationären und teilstationären Leistungen nach KHEntgG und KHG vergütet werden. Zugleich normiert das KHG zur Zielerreichung der wirtschaftlichen Sicherung der Krankenhäuser ( 11 Abs. $1 \mathrm{KHG}$ ) die Mittel der Krankenhausplanung ( $§ 6 \mathrm{KHG}$ ) und der Investitionsförderung ( $\S 8 \mathrm{f}$. KHG). Im Zeichen seiner Komplexität und besonderen Merkmale sowie angesichts normativer Anknüpfungen an die Aufnahme der Krankenhäuser in den Krankenhausplan hat sich das Recht der Krankenhausplanung, 419 „rechtlich ein Unterfall des Rechts der Krankenhausfinanzierung, in der Praxis... »verselbständigt «". 420 Daneben fungieren als (kleinere) Teilbereiche gemäß des dualistischen Finanzierungssystems das Recht der Investitionsförderung sowie das Pflegesatzrecht und ergänzende Regelungen für die vor- und nachstationären Leistungen sowie die Formen der ambulanten Behandlung im Krankenhaus.

Gem. § 8 Abs. 1 S. 1 KHG besteht zunächst ein Rechtsanspruch der Krankenhäuser auf (Investitions-)Förderung, ,soweit und solange sie in den Krankenhausplan eines Landes und bei Investitionen nach $\S 9$ Abs. 1 Nr. 1 in das Investitionsprogramm aufgenommen sind." $\mathrm{Zu}$ einem konkreten Rechtsanspruch auf Bewilligung aber verdichtet sich der mit der Aufnahme in den Krankenhausplan bzw. der Feststellung verliehene Status des förderfähigen und zu fördernden Krankenhauses jedoch erst, wenn die übrigen Voraussetzungen für die Förderung erfüllt sind. Vorher besteht lediglich „eine Art

417 Vgl. das Bayerische Krankenhausgesetz (BayKrG) i.d.F. vom 28. März 2007, BayGVBl. 2007, S. $288 \mathrm{ff}$.

418 Vgl. § 1 Abs. 2 KHEntgG.

419 Dazu unter I.3.4.3.

420 Quaas, in: Wenzel (Hrsg.), Handbuch des Fachanwalts Medizinrecht (2007), Kap. 12, S. 1115. 
Anwartschaft auf Förderung“. 421 In $\S 2$ Nr. 2 KHG ist bestimmt, welche Kosten als Investitionskosten anzusehen sind und welche Kosten diesen gleichgestellt sind (Nr. 3). Die allgemeinen Fördertatbestände für Investitionskosten sind in $\S 9$ KHG bundesrechtlich vorgegeben, ${ }^{422}$ wobei gem. $\S 9$ Abs. $3 \mathrm{KHG}$ für bestimmte Investitionen (Wiederbeschaffung kurzfristiger Anlagegüter; kleine bauliche Maßnahmen) eine Pauschalförderung durch feste jährliche Beträge vorgesehen ist. Gerade vor diesem Hintergrund die Bemessung erfolgt nach Maßgabe des KHG und des Landesrechts ( $§ 9$ Abs. 5 KHG) ${ }^{423}$ - wird die ausnahmslos pauschale Errichtungsförderung in NRW durch die sog. „Baupauschale“ kritisiert bzw. gar für verfassungswidrig erachtet. 424 .

Das Pflegesatzrecht für die Vergütung zur Deckung der Betriebskosten ist insbesondere in den $\S \S 16$ ff. KHG geregelt. Pflegesätze sind die Entgelte der Benutzer oder ihrer Kostenträger für stationäre und teilstationäre Leistungen, § $2 \mathrm{Nr}$. 4 KHG. Auch diese dienen als „leistungsgerechte Erlöse“ dem in § $1 \mathrm{KHG}$ postulierten Ziel der wirtschaftlichen Sicherung der Krankenhäuser. Dabei folgt aus § 4 Nr. 2 KHG zum einen, daß Pflegesätze nach Maßgabe des Gesetzes auch Investitionskosten enthalten können, zum anderen, daß Vergütungen für vor- und nachstationäre Behandlung nicht aus Pflegesätzen vergütet werden. Kern der Vergütung nach Pflegesätzen ist das bereits beschriebene Fallpauschalensystem auf der Grundlage der FPV, für die § $17 \mathrm{~b}$ KHG hinsichtlich der Einführung, der Grundzüge und der verfahrensrechtlichen Vorgaben maßgeblich ist. Gem. $\S 17 \mathrm{~b}$ Abs. 1 S. 3 KHG werden Entgelte nach dem durchgängigen, leistungsorientierten und pauschalierenden Vergütungssystem für die allgemeinen vollund teilstationären Krankenhausleistungen für einen Behandlungsfall vergütet. Weitere entgeltspezifische Vorgaben und solche für die konkreten Schritte in der Konvergenzphase bis zur Erreichung eines landesweiten Durchschnitts regelt das KHEntgG. Dieses bestimmt auch, inwieweit bundeseinheitliche Regelungen über Zu- oder Abschläge vereinbart werden könnten, vgl. § 5 KHEntgG, da und soweit sich manche Leistungen etwa in kleineren Krankenhäusern nicht mehr rechnen, sie demnach in wirtschaftlicher Hinsicht an sich als Finanzierungstatbestände nicht vorliegen, aber im Zeichen der Notwendigkeit flächendeckender Versorgung dennoch vorgehalten werden müssen. $\S 6$ KHEntgG nimmt bestimmte Sachverhalte von der Vergütungsfähigkeit nach dem DRGSystem aus, u.a. soweit sie ab 2005 im DRG-System noch nicht sachgerecht vergütet werden können, und erklärt für die insofern erforderliche Bildung einer Erlössumme die BPflV für anwendbar (§ 6 Abs. 3 S. 2 KHEntgG). Auch für die grundsätzlich gem. § 17 Abs. 1 S. 1 HS. 2 KHG nicht in das DRG-System einbezogenen voll- und teilstationären Krankenhausleistungen gilt die BPflV (vgl. § 1 Abs. 1), auf deren Grundlage die allgemeinen Krankenhausleistungen nach Pflegesätzen vergütet werden (§ 10 Abs. 2 KHG).

421 Quaas, a.a.O., S. 1126 Rn. 24.

422 Zu den Einzelheiten Quaas, in: ders./Zuck, Medizinrecht (2008), § 25 Rn. 56 ff.

423 Näher Quaas, a.a.O., Rn. $112 \mathrm{ff}$.

424 Degener-Hencke, Zum Recht der Krankenhausinvestitionsförderung, NZS 2009, S. 6 ff.; s. auch Quaas, a.a.O., v.a. Rn. 145 f. 
Unter Wettbewerbsgesichtspunkten von besonderer Relevanz ist die Vorgabe, daß die Entgelte für die allgemeinen (notwendigen) ${ }^{425}$ Krankenhausleistungen für alle Benutzer des Krankenhauses einheitlich zu berechnen und zu vereinbaren sind; eine Differenzierung zwischen Versichertenstatus oder Konstenträger findet nicht statt (vgl. $\S \S 8$ Abs. 1 S. 1 KHEntgG, § 17 Abs. 1 S. 1 KHG, § 14 Abs. 1 HS. 1 BPflV). Im übrigen ist auch die Vergütung für die vor- und nachstationäre Behandlung nach $\S 115 \mathrm{a}$ SGB V für alle Benutzer des Krankenhauses einheitlich zu berechnen (§ 17 Abs. 1 S. 1 KHG, $\S 1$ Abs. 3 BPflV).

Andere als die allgemeinen Krankenhausleistungen dürfen „als Wahlleistungen gesondert berechnet werden, wenn die allgemeinen Krankenhausleistungen durch die Wahlleistungen nicht beeinträchtigt werden und die gesonderte Berechnung mit dem Krankenhaus vereinbart ist." 426 Da allerdings auch Wahlleistungsentgelte begrifflich Pflegesätze sind, ${ }^{427}$ müssen sie ebenfalls für alle Benutzer einheitlich berechnet werden. Ein Unterschied zu den allgemeinen Krankenhausleistungen besteht v.a. im Hinblick auf die ohne Berücksichtigung der Kostenträger maßgebliche Preisvereinbarung zwischen Krankenhaus und Patienten. ${ }^{428}$ Über die dennoch vom Pflegesatzrecht geforderte Einheitlichkeit der Tariffierung hinaus finden in weitem Umfang Bestimmungen der Gebührenordnung für Ärzte (entsprechend) Anwendung. 429

Schließlich sind auch sozialrechtliche Regelungen (des Leistungserbringungsrechts) im Kontext der Krankenhausfinanzierung zu benennen. Kollektive Vergütungsvereinbarungen nach gesonderten Maßstäben sind für die vor- und nachstationäre Behandlung im Krankenhaus ( $§ 115 \mathrm{a}$ SGB V) und das ambulante Operieren im Krankenhaus (§ 115b SGB V) zu vereinbaren. Ambulante Behandlungsleistungen durch Krankenhäuser bei Unterversorgung ( $§ 116 \mathrm{a}$ SGB V) werden entsprechend $\S 120$ Abs. 1 SGB V nach den für Vertragsärzte geltenden Grundsätzen aus der vertragsärztlichen Gesamtvergütung vergütet. Selektive Verträge sind ferner (nur noch) bei Verträgen über die Erbringung ambulanter Leistungen nach $\S 116 \mathrm{~b}$ Abs. 1 SGB V bei Teilnahme des Krankenhauses an der Durchführung eines DMP nach § 137g möglich, Vergütungsvereinbarungen eingeschlossen. ${ }^{430}$ Schließlich können im Rahmen der IV Vergütungen autonom zwischen Krankenkassen und Leistungserbringern vereinbart werden $(\S 140 \mathrm{c}$ Abs. 1 SGB V). Soweit dies dem Sinn und der Eigenart der IV entspricht, können die Verträge Abweichendes von den Vorschriften des KHG und des KHEntgG regeln (vgl. $\S 140 \mathrm{a}$ Abs. 4 S. 1 SGB V). Bis zum Ablauf der Anschubfinanzierung wurden daraus gem. $§ 140 d$ Abs. 4 SGB V nur Leistungen finanziert, die nicht dem Budget aus DRG-

425 Zur Definition $\S \S 2$ Abs. 2 KHEntgG, § 2 Abs. 2 BPflV.

$426 \S 17$ Abs. 1 S. 1 KHEntgG; Hervorhebung nicht im Original.

427 Quaas, in: Wenzel (Hrsg.), Handbuch des Fachanwalts Medizinrecht (2007), Kap. 12, S. 1137 Rn. 83.

428 Vgl. Quaas, a.a.O.

429 Quaas, a.a.O.

$430 \S 116 \mathrm{~b}$ Abs. 5 SGB V jedenfalls gilt nicht, s. auch Kingreen, in: Beck-OK SGB V, § 116b Rn. 4. 
Erlösen (§§ 3, 4 KHEntgG) oder der BPflV zuzuordnen sind. ${ }^{431}$ Das KHRG schließlich stellt mit $\S 4$ Abs. 1 S. 2 Alt. 4 KHEntgG nunmehr klar, ,dass die nach $\S 140$ c SGB V zu finanzierenden Leistungen der integrierten Versorgung nicht Bestandteil des Erlösbudgets nach dem Krankenhausentgeltgesetz sind“. 432

\subsubsection{Aktuelle Entwicklungen im Zuge des KHRG}

Das bereits mehrfach erwähnte KHRG enthält im Grunde ein Bündel von Einzelmaßnahmen, weniger jedoch tiefgreifend wettbewerbsorientierte strukturelle Änderungen. Es dient v.a. der wirtschaftlichen Verbesserung der finanziellen Situation der Krankenhäuser und soll diese unmittelbar in Höhe von rund $€ 2,2 \mathrm{Mrd}$. entlasten; 433 weitere Mehreinnahmen in Höhe von $€ 1,9$ Mrd. folgen aus bereits zuvor gesetzlich verankerten Maßnahmen, ${ }^{434}$ wobei im Rahmen der Beratungen des Schätzerkreises zur erstmaligen Festlegung des einheitlichen Beitragssatzes eine Begrenzung auf $€$ 3,5 Mrd. vorgenommen wurde. 435

Im großen und ganzen lassen sich die Änderungen drei Bereichen zuordnen, nämlich dem pauschalierenden Entgeltsystem, der Investitionsfinanzierung sowie sonstigen gesetzlichen Anpassungen zur Gewährung der Leistungsfähigkeit der Krankenhausversorgung. Schwerpunktmäßige Maßnahmen des Gesetzes sind: 436

- Möglichkeit einer Investitionsförderung durch leistungsorientierte Investitionspauschalen ab 1.1.2012 für Plankrankenhäuser; für in den Plan aufgenommene psychiatrische und psychosomatische Einrichtungen ab 1.1.2014; zunächst Entwicklungsauftrag (vgl. § 10 Abs. $1 \mathrm{KHG);}$

- Verlängerung der Konvergenzphase ( 55 Abs. 6 KHG);

- Anteilige (Re-)Finanzierung der Tariflohnerhöhungen für das Jahr 2009 betreffend Erhöhungen für die Jahre 2008 und 2009 (vgl. § 10 Abs. 5 KHEntgG);

- Planmäßiger Wegfall des Abschlags in Höhe von 0,5\% vom Rechnungsbetrag bei gesetzlich Krankenversicherten ( 8 Abs. 9 KHEntgG);

- Einführung eines Förderprogramms zur Verbesserung der Situation des Pflegepersonals (vgl. v.a. § 4 Abs. $10 \mathrm{KHEntgG);}$

- Schrittweise Angleichung der unterschiedlich hohen Landesbasisfallwerte an einen einheitlichen Basisfallwertkorridor im Zeitraum von 2010 bis 2014 (vgl. v.a. $\S 10$ Abs. 8 KHEntgG);

- Einführung eines pauschalierenden tagesbezogenen Entgeltsystems für psychiatrische und psychosomatische Einrichtungen ab dem Jahr 2013 (§ 17d KHG);

431 Vgl. oben I. 2.1.3.2; s. zur Finanzierung ab 2009 unten II.3.1.4.3.

432 BT-Drs. 16/11429 (elektron. Vorabfassung), S 58; Hervorhebung nicht im Original.

433 A.a.O., S. 2.

434 Rau, Regelungen des Krankenhausfinanzierungsreformgesetzes, das Krankenhaus 2009, S. 198.

435 PM des BMG v. 18.12.2008; Rau, a.a.O., S. $201 \mathrm{f}$.

436 Vgl. BT-Drs. 16/11429, S. 1 f. 
- Aufhebung von Regelungen der zum Jahresende 2008 auslaufenden DRGEinführungs- und Konvergenzphase sowie Aktualisierung und Ergänzung verbleibender Regelungen für den künftigen Regelbetrieb des DRGVergütungssystems.

Auf Details kann hier nur bedingt eingegangen werden. Im Hinblick auf den Untersuchungsgegenstand sind zwei Gesichtspunkte kurz herauszugreifen: die Investitionsförderung durch Pauschalen und die Verlängerung der Konvergenzphase:

Von besonderem Interesse ist die zukünftig optionale Investitionsförderung durch leistungsorientierte Investitionspauschalen deshalb, weil der damit verbundene Entwicklungsauftrag tw. als Überbleibsel der Forderung nach einer monistischen Krankenhausfinanzierung, 437 tw. als „Übergangsmodell zur Ersetzung der dualen Finanzierung durch eine monistische Krankenhausfinanzierung" bezeichnet wird. ${ }^{438}$ So könnte

„in einem weiteren Schritt die Einzelförderung vollständig abgeschafft und die Funktion der Länder in eine bloße Zahlstelle umgewandelt werden..., wenn eines Tags die absolute Höhe der Investitionspauschalen in Euro auf Bundesebene bzw. eine Kopplung an einen bundeseinheitlichen DRG-Basisfallwert vorgegeben und im letzten Schritt die „Zahlstelle Länder“ mit entsprechender finanzieller Kompensation der Länder durch die „Zahlstelle Krankenkasse“ ersetzt wird“. 439

Wesentlich ist zwar zunächst, daß das Recht der Länder unberührt bleibt, nach ihrer Wahl weiterhin durch Einzelförderung von Investitionen einschließlich der Pauschalförderung kurzfristiger Anlagegüter zu entscheiden, § 1 Abs. 1 S. 4 KHG. Kommt es jedoch nach Entwicklung der „Grundsätze... für die Ermittlung eines Investitionsfallwertes auf Landesebene“ und der „Grundstrukturen für... Investitionsbewertungsrelationen“ zur Kalkulation bundeseinheitlicher Investitionsbewertungsrelationen durch das DRG-Institut, sind die Länder entsprechend gebunden: Sie vermögen dann zwar noch die Höhe der Gesamtfördermittel, nicht aber die der Pauschale autonom festzusetzen. ${ }^{440}$

Die Verlängerung der Konvergenzphase um ein Jahr, finanzwirksam verteilt auf 2009 und 2010, ist vor allem kontextbezogen zu betrachten. Insofern ist und war es jedenfalls konsequent, vom Vorhaben der Budgetkonvergenz nicht abzuweichen und damit Planungssicherheit zu schaffen. Das zielgerichtete Agieren in der Konvergenzphase und Anstrengungen v.a. derjenigen, die Anteile abzugeben hatten, wären unter nicht unerheblichen Verwerfungen ,frustriert“" worden, wären die Preise durch das KHRG alsdann weithin freigegeben worden. Dessen ungeachtet wurde die Verlängerung der Konvergenzphase in den Dienst der Begrenzung der Mehraufwendungen auf $€$ 3,5 Mrd. gestellt, ${ }^{441}$ um Mehrbelastungen durch einen ungleichgewichtigen letzten Konvergenz-

437 Rau, Regelungen des Krankenhausfinanzierungsreformgesetzes, das Krankenhaus 2009, S. 198 (204).

438 Degener-Hencke, Zum Recht der Krankenhausinvestitionsförderung, NZS 2009, S. 6 (13).

439 Degener-Hencke, a.a.O.

440 Degener-Hencke, a.a.O.

441 Rau, Regelungen des Krankenhausfinanzierungsreformgesetzes, das Krankenhaus 2009, S. 198 (202). 
schritt in Höhe von geschätzten $€ 600-800$ Mio. zu vermeiden. ${ }^{442}$ Die Umsetzung der Verlängerung erfolgt angesichts der bereits im Jahre 2009 maßgeblichen Landesbasisfallwerte mittels hälftiger Aufteilung des Konvergenzsaldos auf 2009 und 2010 und die Korrektur über Zu- bzw. Abschläge. ${ }^{443}$

\subsubsection{Krankenhausplanungsrecht}

Die Krankenhausplanung als Teil des Krankenhausfinanzierung ist Instrument zur wirtschaftlichen Sicherung der Häuser und bedarfsgerechten Versorgung der Bevölkerung, $\S \S 6,1$ KHG. Sie ist für die wirtschaftliche und die wettbewerbliche (Ausgangs-) Situation von besonderer Relevanz. Die Aufnahme eines Krankenhauses in den Krankenhausplan, deren Aufstellung Aufgabe der Länder ist ( 6 Abs. $1 \mathrm{KHG}$ ), fungiert gem. $\S 8$ Abs. $1 \mathrm{KHG}$ als zentrale Voraussetzung der Investitionsförderung. Bei den aufgenommenen Plankrankenhäusern (§ 108 Nr. 2 SGB V), gilt"“ die Aufnahme in den Krankenhausplan gem. § 109 Abs. 1 S. 2 SGB V als Versorgungsvertrag. Mittels dieses (fingierten) Versorgungsvertrages wird das Krankenhaus zur Versorgung der Versicherten zugelassen, § 109 Abs. 4 S. 1 SGB V. Es ist zur Behandlung ebenso verpflichtet (S. 2) wie die Kassen zur Verhandlung der Pflegesatzvereinbarungen (S. 3). Die Aufnahme in den Krankenhausplan bewirkt daher „eine Mindestgarantie wirtschaftlicher Sicherheit". 444

Die inhaltlichen Vorgaben der Planaufstellung trifft in weitem Umfang das Landesrecht ( $\$ 6$ Abs. 4 KHG). Allerdings ergeben sich auch aus dem Bundesrecht wichtige Anforderungen, erschlossen aus dem Zusammenhang des KHG und entwickelt auf der Grundlage des $\S 6$ Abs. 2 KHG a.F.: ${ }^{445}$ So muß die Planung Ziele festlegen, eine Analyse des Bedarfs der Bevölkerung an Krankenhausbetten, räumlich, fachlich und nach Versorgungsstufen gegliedert (Bedarfsanalyse) sowie eine Analyse zur Eignung bzw. der tatsächlichen Versorgungsbedingungen bei den vorhandenen Krankenhäusern (Krankenhausanalyse) enthalten. ${ }^{446}$ Vor diesem Hintergrund ist darüber zu entscheiden, welche Krankenhäuser per zusammenfassender Aufstellung in den Krankenhausplan aufgenommen werden (Versorgungsentscheidung). Gem. der Vorgabe des $\S 7$ Abs. 1 KHG sind bei der Krankenhausplanung und der Aufstellung der Investitionsprogramme einvernehmliche Regelungen mit den Beteiligten - v.a. den Krankenhäusern - anzustreben (S. 2). Das betroffene Krankenhaus ist anzuhören (S. 1 Hs. 2).

Der Krankenhausplan ist keine Rechtsnorm und nicht als solcher angreifbar. ${ }^{447}$ Die Aufnahme oder Nichtaufnahme (bzw. Herausnahme) des einzelnen Krankenhauses in

442 BT-Drs. 16/11429, S. 59f.

443 A.a.O.; § 5 Abs. 6 KHEntgG.

444 Burgi, Konkurrentenschutz in der Krankenhausplanung, NZS 2005, S. 169 (170).

445 BVerwGE 72, 38 (46 ff.); Quaas, in: Wenzel (Hrsg.), Handbuch des Fachanwalts Medizinrecht (2007), Kap. 12, S. 1146 f.

446 BVerwG, Urt. v. 25.9.2008, Az. 3 C 35/07, GesR 2009, S. 27 (28).

447 BVerwGE 72, 38 (45); BVerfGK 3, 39 (40 m.w.N.). 
den Krankenhausplan wird erst „auf der zweiten Stufe“ - durch Bescheid seitens der in der Regel nachgeordneten Behörde festgestellt, $\S 8$ Abs. 1 S. 3 KHG. Ein Anspruch auf Feststellung der Aufnahme in den Krankenhausplan besteht nicht (§ 8 Abs. 2 KHG), wobei diese Bestimmung in erster Linie auf die pflichtgemäße Auswahl zwischen mehreren Krankenhausträgern rekurriert, die in einer „ersten Stufe“ bereits als für eine bedarfsgerechte Versorgung in Frage kommend anzusehen waren. ${ }^{448}$

Die Feststellung dann ist Verwaltungsakt und gem. § 8 Abs. 1 S. 4 KHG im Verwaltungsrechtsweg angreifbar. Je nach Klageziel des Vorgehens gegen die Aufnahme in den oder Herausnahme aus dem Krankenhausplan kommen die Verpflichtungs- bzw. Anfechtungsklage in Betracht. Darüber hinaus hat die verfassungsrechtliche, an der Berufsausübungsfreiheit orientierte Bedeutung der Entscheidung dazu geführt, daß auch Klagen von Konkurrenten grundsätzlich zulässig und nicht allein mit Blick auf eine Willkürkontrolle überprüfbar sind. ${ }^{449}$

\subsubsection{Leistungsrecht und Leistungserbringungsrecht}

Schließlich enthält das SGB V diverse krankenhausrechtliche Regelungen. Leistungsrechtlich etwa umfaßt die Krankenbehandlung auch Krankenhausbehandlung, § 27 Abs. 1 S. 2 Nr. 5 SGB V. Sie darf nur verordnet werden, soweit eine ambulante Versorgung nicht ausreicht, § 73 Abs. 4 S. 1 SGB V. Die zentralen Leistungsvoraussetzungen der voll-, teil-, vor- und nachstationär sowie (auch) ambulant erbrachten Krankenhausbehandlung regelt $\S 39$ SGB V, dem zufolge die vollstationäre Behandlung gegenüber den anderen Behandlungsarten zurücktritt, soweit das Behandlungsziel durch diese erreicht werden kann (§39 Abs. 1 S. 2 SGB V). Darüber hinaus lassen sich das Recht der Zulassung der Krankenhäuser ( $§ 107$ ff. SGB V), das Recht der (statusbegründenden) Versorgungsverträge mit den Krankenhäusern ( $\S 109$ ff. SGB V), das Recht der Beziehungen zu Krankenhäusern im Schnittstellenbereich zur vertragsärztlichen Versorgung ( $\S 115$ ff. SGB V) sowie - grob gefasst - das Recht der Qualitätssicherung (insbes. $\S § 137 \mathrm{ff}$. SGB V) und - für alle Leistungserbringer gültig - der integrierten Versorgung ( $\S 140 \mathrm{a}$ ff. SGB V) benennen.

\subsection{Kostenträger}

Ungeachtet der Kostenträger außerhalb der GKV, etwa der Träger der Sozialhilfeoder der Unfallversicherung, tragen neben den privaten Krankenversicherungsunternehmen insbesondere die gesetzlichen Krankenkassen die (Last der) Kosten. Der Begriff „Kostenträger“ wird der Rolle der Krankenkassen nur zum Teil gerecht, sind Sie,

448 BVerfGK 3, 39 (40 f. m.w.N.); Quaas, in: Wenzel (Hrsg.), Handbuch des Fachanwalts Medizinrecht (2007), Kap. 12, S. 1150 f. m.w.N.

449 Näher unter I.4.3.1. sowie insbesondere unter II.4.1. 
wie gezeigt, doch auch Preis- und Leistungsgestalter und als Konkurrenten im Gesundheitsmarkt aktiv.

Für die GKV wurde bereits erwähnt, daß die Zahl der Krankenkassen von mehr als 1.209 Krankenkassen im Jahr $1991^{450}$ auf nur noch 202 zum 1. Januar 2009 gesunken ist. ${ }^{451}$ Der Fusionsprozeß dieser in einzelne Kassenarten gegliederten ${ }^{452}$ Körperschaften des öffentlichen Rechts wird zukünftig weiter forciert durch Ermöglichung der „kassenartübergreifenden Vereinigung der Krankenkassen“, § 171a SGB V. Der historisch bedingten Einteilung der Krankenkassen in Primärkassen und Ersatzkassen (zu letzteren $\S \S 168$ ff. SGB V) folgt die Einteilung der Primärkassen in Ortskrankenkassen ${ }^{453}$ ( $\S$ 143 ff. SGB V), Betriebskrankenkassen ${ }^{454}$ (§§ 147 SGB V), Innungskrankenkassen 455 ( $\S 157$ SGB V) Ersatzkassen 456 ( $§ 168$ SGB V), die Knappschaft Bahn See ( 1167 SGB V) 457 und landwirtschaftliche Krankenkassen 458 (§ 166 SGB V). Ortskrankenkassen bestehen für abgegrenzte Regionen, die sich allerdings - wie die AOK Rheinland Hamburg - auch auf mehrere Länder strecken können, vgl. § 143 Abs. 3 SGB V. Betriebskrankenkassen existieren als auf die Versicherung von Betriebsangehörigen beschränkte (,geschlossene“) oder allgemein geöffnete Krankenkassen, wobei das Personal entweder vom Arbeitgeber oder von der Krankenkasse selbst angestellt ist.

Für die Verfassung der Krankenkassen gelten v.a. §§ 29 ff. SGB IV, soweit nicht krankenversicherungsrechtliche Besonderheiten bestehen. Die Krankenkassen werden durch Vorstände geführt, $\S \S 35,35 \mathrm{a}$ SGB IV. Das Organ der Selbstverwaltung ist der Verwaltungsrat, vgl. § 31a Abs. 3 SGB IV. Die Versicherungsträger geben sich eine Satzung, § 34 SGB IV.

Die Krankenkassen bilden auf Landesebene Landesverbände ( $§ 207$ ff. SGB V), ebenfalls Körperschaften des öffentlichen Rechts, die ihrerseits nach Kassenarten vormals jeweils einen Bundesverband bilden, §§ 212 SGB V a.F., die als Spitzenverbände der Krankenkassen agierten. ${ }^{459}$ Auch nach der Reform des GKV-WSG sind die Lan-

450 BMG, Pressemitteilung vom 24. Juni 2006, eingestellt am 25.5.2006, abrufbar unter www.bmg.bund.de (Presse, Archiv, Presse 2/2006, 25.6.2006: „Ulla Schmidt begrüßt erste länderübergreifende AOK-Fusion“).

451 BMG, Ergebnisse der GKV-Statistik KM1, Stand: 10. März 2009.

$452 \S 4$ SGB V; $\S \S 21$ Abs. 1 SGB I, $\S 1$ und 2 SGB V.

453 Nach Ergebnissen der GKV-Statistik KM1 (hrsgg. vom BMG, Stand: 10. März) waren dies im Januar 200915 (alle landesunmittelbar und regional geöffnet).

454 Januar 2009 (a.a.O.): insgesamt 155, davon 62 landes- und 93 bundesunmittelbar sowie 75 regional, 32 bundesweit und 48 nicht geöffnet.

455 Januar 2009 (a.a.O.): insgesamt 14, davon 8 landes- und 6 bundesunmittelbar sowie 11 regional und 3 bundesweit geöffnet.

456 Es handelt sich um eine bundesunmittelbare, nicht geöffnete Kasse.

457 Januar 2009 (a.a.O.): insgesamt 8 bundesweit geöffnete Kassen (1 landes- und 7 bundesunmittelbar).

458 Dies waren im Januar 2009 (a.a.O.) insgesamt 9 nicht geöffnete Kassen, von denen 7 landes- und 2 bundesunmittelbar waren.

459 Vgl. zu deren Fortbestand und Rechtsnachfolge $\S 212$ f. SGB V m.W.v. 1.1.2009. 
desverbände (wie die Krankenkassen selbst) zum Vertragsschluß mit zugelassenen Krankenhäusern über die ambulante Versorgung im Krankenhaus nach $\S 116 \mathrm{~b}$ Abs. 1 SGB V zuständig. Demgegenüber ist seit dem 1. Juli 2008 der neue Spitzenverband Bund der Krankenkassen ( $\S 217$ a ff. SGB V), Partner der Vereinbarung der Fallpauschalen nach $\S 17 \mathrm{~b}$ Abs. 2 KHG. ${ }^{460}$

Weiterer Partner dieser Vereinbarung ist wegen des finanzierungsrechtlichen Gleichlaufs der Vergütung der allgemeinen Krankenhausleistungen auch der Verband der privaten Krankenversicherung. Ihm gehörten Ende 2007 insgesamt 47 Mitgliedsunternehmen an, davon 21 Versicherungsvereine auf Gegenseitigkeit (mit 45\% der Gesamtbeitragseinnahmen und 52,3\% der Gesamtzahl an Vollversicherten) und 26 Aktiengesellschaften (55\% bzw. 47,7\%). ${ }^{461}$

\section{Wettbewerb und Gesundheitsversorgung}

\subsection{Die Idee des Wettbewerbs}

Wettbewerb ist Ausdruck wirtschaftlich orientierten Verhaltens der Marktteilnehmer und als Prozess zu begreifen. Auch wenn es verbreiteter Erkenntnis entspricht, daß Wettbewerb als reales Phänomen einer Definition kaum zugänglich ist, 462 entbindet dies nicht von der Aufgabe, Grundvorstellungen und Steuerungsansätze in normativen Bezugspunkte(n) aufzuzeigen. Kennzeichnend ist meist - ohne eine „neoklassische“ Ausprägung zu präjudizieren - die Freiheit der Marktteilnehmer, d.h. Handlungs- und Entscheidungsfreiheit, mindestens jedoch beschränkt durch dieselbe des Konkurrenten. In diesem Sinne verlangt und ermöglicht Wettbewerb „einen Mindestgrad an wirtschaftlicher Freiheit“ 463 der Marktteilnehmer und generiert Wohlstand als Ergebnis ökonomisch erfolgreich angelegter freier Entscheidungen. Unter konstitutionellen Gesichtspunkten erweist sich die Freiheit wirtschaftlicher Betätigung als normativ geschütztes Substrat der Verfassungs- und Wirtschaftsordnung, auch wenn die Verfassung - im „übrigen“- „wirtschaftspolitisch neutral“ angelegt ist. ${ }^{464}$

Demgemäß kommt „Wettbewerb“ in einer freiheitlichen Gesellschaft mit freiheitlicher Wirtschaftsordnung ein Eigenwert zu. ${ }^{465}$ Der Wert als solcher allerdings ist kein Sollensgebot, sondern kennzeichnet die Bewertung von Zuständen - oder Phänomenen

460 S. Art. 18 Nr. 4 b GKV-WSG (BGBl. I 2007, S. 445 f.), der am 1. Juli 2008 in Kraft tritt, Art. 46 Abs. 9 (a.a.O., s. 472).

461 Verband der privaten Krankenversicherung e.V. (Hrsg.), Die private Krankenversicherung. Rechenschaftsbericht 2005, S. 10; abrufbar unter www.pkv.de (wir über uns).

462 Köhler, in: Hefermehl/Köhler/Bornkamm, Wettbewerbsrecht, 25. Aufl. 2007, UWG Einl. Rn. 1.6.

463 Köhler, a.a.O., UWG Einl. Rn. 1.15. m.w.N.

464 BVerfGE 4, 7, 17 f.; 50, 290, 336 ff.

465 Cassel (2003), in: Arnold/Klauber/Schellschmidt (Hrsg.), Krankenhaus-Report 2002, S. 3 (4 m.w.N.). 
- die in einem Sinne gutgeheißen werden, daß sie als tragfähiger Konsens für eine Verfassung des Gemeinwesens fungieren. Die Grundentscheidung für Wettbewerb der zunächst frei agierenden Marktteilnehmer wird gleichzeitig zur Ordnungsvorstellung.

\subsection{Wettbewerb im Gesundheitswesen}

Im Recht der Krankenversicherung 466 - vor allem im Recht der GKV - unterliegt die Konzeption von Wettbewerb in besonderer Weise einer spezifisch funktionalen Ausrichtung in Form der allgemeinen Gesundheitsversorgung. Wettbewerbsorientiertes Handeln soll danach in Diensten, mindestens aber im Einklang mit dem nach § 1 SGB V tragenden Prinzip der Solidarität stehen. Ungehinderter Marktzutritt, Preisfreiheit, Freiheit in der Gestaltung der Produkte und Dienstleistungen u.a.m. sind mit der allgemeinen solidarischen Gesundheitsversorgung weithin unvereinbar. Darüber hinaus ergeben sich Probleme auch deshalb, weil der Patient als „Nachfrager“ mit einem im Grundsatz rational handelnden Konsumenten bedingt vergleichbar ist, sei es, weil insbesondere im Gesundheitswesen Informationsasymmetrien zutage treten, weil der Patient im Zustand der Hilfebedürftigkeit nur bedingt frei entscheidet, oder schließlich, weil er - in aller Regel - nicht derjenige ist, der die Leistung unmittelbar bezahlt. Dies und ein im Grunde anerkanntes besonderes Regulierungsbedürfnis führen dazu, daß gerade das Gesundheitswesen (a priori) als unvollkommener Markt betrachtet wird. „Die Berufsausübung des Vertragsarztes“, so das $B \operatorname{Verf} G$, „findet in einem staatlich regulierten Markt statt.“467 Das schließt jedoch nicht aus, daß sich Unterschiede relativer Marktvollkommenheit in einzelnen Teilmärkten bzw. Marktsegmenten ausmachen lassen, wobei die Marktöffnung ihrerseits Ausdruck ordnungspolitischer Vorstellungen ist.

In der Wirtschaftsordnung eines begrenzten und dem Grundsatz der Solidarität zur Sicherung allgemeiner Gesundheitsversorgung verpflichteten Marktes erscheint die normative Sicherung und Durchsetzung der Wettbewerbsfreiheit weithin als nachgeordnete Zielvorstellung zur Erreichung der Leistungsziele. Selbst in dieser funktionalen Ausrichtung aber wird dem Wettbewerb durchaus Eigenwert zuerkannt. Der „Regulierung" hingegen kommt kein Eigenwert zu, welcher auch nicht dadurch vermittelt wird, daß Regulierung entweder der allgemeinen Gesundheitsversorgung oder gar der Sicherung des Wettbewerbs selbst zu dienen bestimmt ist. Danach ist die oft konstatierte Dichotomie von "Wettbewerb und Regulierung“ eine solche auf der Ebene instrumenteller oder operativer Verfahren und Spielräume, während das wert- und zielorientierte Be-

466 Becker, Gesetzliche Krankenversicherung zwischen Markt und Regulierung, JZ 1997, S. 534 ff.; Steinmeyer, Wettbewerbsrecht im Gesundheitswesen (2000); Schmehl/Wallrabenstein: Steuerungsinstrumente im Recht des Gesundheitswesens, Bd. 1 Wettbewerb (2005). Vgl. zum „Wettbewerb in der Gesetzlichen Krankenversicherung“ aus gesundheitsökonomischer Sicht die Beiträge bei Knappe (Hrsg.), Wettbewerb in der gesetzlichen Krankenversicherung (1999), und Wille (Hrsg.), Zur Rolle des Wettbewerbs in der gesetzlichen Krankenversicherung (1999).

467 BVerfG, Kammer, Beschl. v. 17.8.2004, NZS 2005, S. 144 (146); BVerfGE 103, 172 (185 f.). 
griffspaar als „Solidarität und Wettbewerb“ zu begreifen ist, dessen funktionale Synthese mit der Leitvorstellung von der „solidarischen Wettbewerbsordnung“ zu konzeptionieren versucht wird. 468

Dieses Verständnis allerdings ist das Produkt erst der jüngeren Zeit. Das gilt v.a. für den Leistungswettbewerb im engeren Sinne und den Vertragswettbewerb zur Schaffung neuer Strukturen der Leistungserbringung. Noch vor gut 12 Jahren gab es lediglich beschränkten Wettbewerb jeweils auf Seiten der Kostenträger oder der Leistungserbringer, der sich weithin auf das nach innen gerichtete Streben nach eigener Effizienz bei noch fehlender Diversifikation von Leistung und Leistungserbringung richtete.

Der 1996 eröffnete Mitgliederwettbewerb der Krankenkassen erfolgte vor dem Hintergrund eines im Grunde nicht existenten „Produktwettbewerbs“; leistungsrechtliche Diversifikationen waren kaum möglich; Anreize wie Boni für gesundheitsbewusstes Verhalten ( $\$$ 65a SGB V) kamen erst seit dem Jahre 2000 zum Tragen. Wettbewerb fand mithin nahezu ausschließlich über den Beitrag statt. Im übrigen spielten eher ,weiche Parameter" eine Rolle: Image und Service wurden als Wettbewerbsfelder identifiziert, ${ }^{469}$ ohne daß der Leistungszuschnitt, sonst essentialium wettbewerbsorientierter Tätigkeit, das Geschehen maßgeblich bestimmen konnte. Erfolgsrelevante Einflussgrößen waren daneben die Risiko- und Verwaltungskostenstruktur der Krankenkasse.

Auf Seiten der Leistungserbringer stand zunächst ebenfalls deren innerorganisatorische Effizienz im Vordergrund; Krankenhäuser wurden zunehmend in privater Trägerschaft geführt bzw. es stellte sich die Frage der Organisation in privatrechtlicher Form. Im hoch regulierten Krankenhausmarkt war es zudem zunächst so, daß nach erfolgreicher Aufnahme in den Krankenhausplan mit anschließendem Kontrahierungszwang ein eher loser Zusammenhang von Vergütung und Performance bestand. Dies änderte sich gründlich mit der Einführung der DRG, aber auch mit der Anknüpfung an Qualitätsvoraussetzungen, wobei beides zu einem verschärften Spezialisierungs- und auch Verdrängungswettbewerb führte.

Die zunehmende Verankerung wettbewerblicher Elemente in der GKV kaprizierte sich alsdann mehr und mehr auf die intersektorale Betrachtung, da und soweit offensichtlich wurde, daß gerade an den Schnittstellen Bedarf zur Beseitigung der bemängelten Unter-, Über- und Fehlversorgung bestand. Der Fokus richtete sich auf die Kooperation, nicht aber eine korporatistische, sondern kooperative Kooperation. Dies gilt nicht nur für Zusammenarbeit und Netzwerke von Leistungserbringern, sondern v.a. für die selektivvertraglich fundierte Zusammenarbeit zwischen Kostenträgern und Leistungserbringern. Kooperieren letztere miteinander, dient dies der (internen) „Vorabintegration" zur Schaffung von Strukturvorteilen im Wettbewerb um Versorgungsstrukturen in Zusammenarbeit mit den Kostenträgern.

468 Vgl. nachfolgend.

469 Mühlhausen, Mitgliederwettbewerb innerhalb der gesetzlichen Krankenversicherung (2002), S. $99 \mathrm{ff}$. 
Ungeachtet dessen steht der Wettbewerb in Diensten des Sozialen: Verankert v.a. im Recht der Leistungserbringung, ${ }^{470}$ erstrebt er in erster Linie wohlfahrtsfördernde Allokationseffekte 471 in Form von „Qualität und Wirtschaftlichkeit im Gesundheitswesen“. Die gesetzlichen Reformwerke der Jahre $1999^{472}$ und $2003^{473}$ haben den Wettbewerb als Strukturmerkmal der Gesetzlichen Krankenversicherung entscheidend fortentwickelt. Im Zeichen neu geschaffener und durchgreifend flexibilisierter Versorgungsstrukturen treten neben die Allokationsfunktion zugleich Innovationseffekte, ${ }^{474}$ und einem solchermaßen funktionierenden Wettbewerb der Leistungserbringer wird das Potential für die maßgeschneiderte Bereitstellung einer an den Bedürfnissen der Patienten ausgerichteten Versorgung zuerkannt. ${ }^{475}$ Damit erscheint mehr und mehr gerechtfertigt, von den gesetzlichen Grundlagen der GKV als einer ,solidarische[n] Wettbewerbsordnung“476 zu sprechen. Dabei löst sich die Idee des Wettbewerbs mitunter von der Fessel ihrer Instrumentalisierung und wird Teil der primären Zielvorstellung, dennoch stets eingebunden in die zentrale Konzeption, Versorgungsstrukturen zu verbessern und sektorale Grenzen der medizinischen Versorgung zu überwinden. ${ }^{477}$

Im Bereich des herkömmlich als eher dirigistisch beschriebenen GKV-Systems darf die Rolle des regulierten Wettbewerbs auch als „Koordinator des Marktgeschehens“478 indes nicht verkannt werden. Eine „neoklassische Konzeption“, die Zielkonflikte mit der Freiheitsverwirklichung der weitgehend unbeschränkt agierenden Akteure leugnet, ${ }^{479}$ erscheint mit den im Gesundheitswesen vorzufindenden Strukturen, Bedürfnissen und Marktverhältnissen kaum vereinbar. Gleichwohl steht einer wettbewerbsorientierten Ausprägung des GKV-Systems nicht entgegen, daß besondere ordnungspolitische Grundbedingungen und eine solidarische Ausgestaltung des Versicherungssystems dem Wettbewerb Grenzen setzen.

470 Siehe bspw. Greß/Ebsen/Haas/Jacobs/Schulze/Szecsenyi/Wasem, Wettbewerbliche Steuerung in der GKV - Perspektiven für mehr Qualität und Wirtschaftlichkeit, SF 2003, S. 105 ff.; Kingreen, Wettbewerbsrechtliche Aspekte des GKV-Modernisierungsgesetzes, MedR 2004, S. 188 ff. Zum (begrenzten) Wettbewerb der Krankenkassen beim Leistungsangebot Mühlhausen, Der Mitgliederwettbewerb innerhalb der gesetzlichen Krankenversicherung (2002), S. 185 ff.

471 Vgl. Cassel (2003), in: Arnold/Klauber/Schellschmidt (Hrsg.), Krankenhaus-Report 2002, S. 3 (5).

472 Gesetz zur Reform der GKV v. 22.12.1999; GKV-GRG, BGBl. I 1999, S. 2626; vgl. dazu BTDrucks. 14/1245, S. 53; hinzu kommt das Ziel der Beitragssatzstabilität.

473 GKV-Modernisierungsgesetz (GMG) v. 14.11.2003, BGB1. I 2003, S. 2189; BT-Drucks. 15/1525, S. $1,71 \mathrm{ff}$.

474 BT-Drucks. 15/1525, S. 74.

475 A.a.O.

476 A.a.O., S. 71. Zur Widerspruchsfreiheit des Grundkonzepts der ,gleichzeitige[n] Verfolgung von Solidarität und Wettbewerb" als Hauptziele[n] des Risikostrukturausgleichs (RSA) zwischen den Krankenkassen BVerfG, Beschl. v. 18.7.2005, 2 BvF 2/01, BVerfGE 113, 167 (233).

477 Zu letzterem BT-Drucks. 15/1525, S. 74.

478 Köhler, in: Hefermeh1/Köhler/Bornkamm, Wettbewerbsrecht, 25. Aufl. 2007, UWG Einl. Rn. 1.13, eigene Hervorhebung.

479 Köhler, a.a.O., Rn. 1.16. 
Ein Optimum an „solidaritätsprinzipskompatiblen“ Markt- und Wettbewerbsstrukturen lässt sich kaum quantifizieren. Wohl aber können einzelne Wettbewerbsmechanismen evaluiert und Kausalitäten ergründet und dadurch Einschätzungen relativiert werden, denen zufolge die Solidarische Wettbewerbsordnung ein „Torso geblieben“ und die GKV lediglich Ausdruck einer „dysfunktionalen Mischung aus dirigistischer und wettbewerblicher Steuerung" sei. 480

\subsection{Verfassungsrechtliche Fundierung}

\subsubsection{Verfassungsrechtlicher Schutz der Formen freier wirtschaftlicher Betätigung}

Die Freiheit wirtschaftlicher Betätigung ist das Substrat verfassungsrechtlicher Schutzgarantien im Bereich wettbewerblicher Betätigung. Nicht alle Akteure indes können sich auf die relevanten Grundrechte 481 berufen. Da sie aufgrund von Kompetenzen und nicht in Ausübung verbürgter Freiheiten tätig werden, sind gesetzliche Krankenkassen als Körperschaften des Öffentlichen Rechts nicht grundrechtsfähig. 482 Demgegenüber genießen die Unternehmen der PKV in personeller Hinsicht den Schutz der Freiheit unternehmerischer Betätigung, soweit diese durch Grundrechte, v.a. die Berufsfreiheit garantiert wird. Gem. Art. 19 Abs. 3 GG gelten Grundrechte auch für inländische juristische Personen, soweit sie - wie die Garantien zur wirtschaftlichen Betätigung - ihrem Wesen nach auf diese Personen anwendbar sind. ${ }^{483}$

Private Krankenhausträger können sich wie kirchliche Einrichtungen und solche der Wohlfahrtspflege auf den Schutz der Grundrechte gem. Art. 19 Abs. 3 GG berufen, der unselbständigen öffentlichen Einrichtungen (in öffentlicher Trägerschaft) versagt bleibt. Werden Krankenhäuser in öffentlicher Trägerschaft betrieben, führt die Wahl der privaten Rechtsform allein nicht zur Eröffnung des personellen Anwendungsbereiches der Grundrechte. Strittiger Beurteilung unterliegt die Annahme der Grundrechtsträgerschaft bei sog. gemischt-wirtschaftlichen Unternehmen. Werden sie vom Staat durch Einflussmöglichkeiten und/oder Anteilseignung nicht beherrscht, wird für den Grundrechtsschutz votiert, der wegen der anderenfalls entstehenden Schutzlücke auch geboten

480 Cassel (2003), in: Arnold/Klauber/Schellschmidt (Hrsg.), Krankenhaus-Report 2002, S. 3 (9).

481 Zum sachlichen Schutzbereich sogleich.

482 Vgl. bereits BVerfGE 39, 302 (316).

483 Ausdrücklich zu Unternehmen der privaten Krankenversicherung BVerfG, 4.2.2004, BVerfGK 2, 283. Zur Berufung auf grundrechtliche Positionen gegen Änderungen durch das GKV-WSG, v.a. die Portabilität der Altersrückstellungen und den Kontrahierungszwang für Verträge im Basistarif, etwa Depenheuer, Verfassungsrechtliche Grenzen der Portabilität der Altersrückstellungen in der Krankenversicherung, Gutachten im Auftrag des Verbandes der PKV (2006); Sodan, Private Krankenversicherung und Gesundheitsreform 2007 (2007), S. 36 ff., 74ff.; Boetius, Gegen den Wind Der Basistarif der Gesundheitsreform bricht Europa- und Verfassungsrecht, VersR 2007, S. 431 ff. 
ist. ${ }^{484} \mathrm{Im}$ Falle der evidenten Beherrschung bzw. des dominierenden Einflusses sprechen - entgegen anderer Auffassung 485 - gute Gründe gegen eine Schutzposition, ${ }^{486}$ da angesichts der organisationsrechtlich in der Regel intendierten Dominanz und Steuerungsmöglichkeit meist keine grundrechtstypische Gefährdungslage besteht, die eine weit reichende Auflösung der Konfusion von Bindung und Berechtigung voraussetzt. Patienten schließlich sind Grundrechtsträger als natürliche Personen, ebenso sind es Leistungserbringer, die sich - unter berufsrechtlichen Restriktionen - zu ebenfalls geschützten Gesellschaften privaten Rechts zusammenschließen können. Eine Relativierung des Grundrechtsschutzes der Vertragsärzte allein durch „Einbindung“ in das öffentlich-rechtliche System, sei es auf Schutzbereichs-, Eingriffs- oder Rechtsfertigungsebene, ist abzulehnen.

Ausprägungen wettbewerbsorientierter Freiheit der wirtschaftlichen Betätigung sind im wesentlichen dem Schutzbereich der Berufsfreiheit, Art. 12 Abs. 1 GG zuzuordnen. Dort, wo dieser sachlich oder wie bei Ausländern (unbeschadet des Schutzes durch Art. 2 Abs. 1 GG) bereits in personeller Hinsicht nicht eröffnet ist, kommt die allgemeine Garantie der (wirtschaftlichen) Handlungsfreiheit zugunsten autonomen Handelns zum Tragen. Die Ausprägungen wirtschaftlicher Betätigungsfreiheit sind damit im wesentlichen Aspekte der Berufs(ausübungs)freiheit. Diese Zuordnung ist hinsichtlich der für die wettbewerbliche Betätigung wichtigen Vertragsfreiheit allerdings umstritten. Die Vertragsfreiheit wird wegen ihrer fundamentalen Bedeutung für die Berufsausübung teilweise im Schutzbereich der Berufsfreiheit verortet, teilweise im Hinblick auf einen eigenständigen materiellen Gehalt und als unbenanntes Freiheitsrecht als vom Schutzbereich des Art. 2 Abs. 1 GG erfaßt angesehen. ${ }^{487}$ Demgemäss wird auch der Schutz der Preisfreiheit qua Zugehörigkeit zur Vertragsfreiheit 488 Art. 2 Abs. 1 GG unterstellt, ${ }^{489}$ während anderer Auffassung zufolge wiederum Art. 12 Abs. 1 GG einschlägig sei. 490 In den Kanon dieser Freiheiten sind auch ,interprivate Verhaltensweisen der Werbung, des Vertriebes und des Absatzes“ einzuordnen. ${ }^{491}$ Dabei vermittelt Art. 12 Abs. 1 GG keinen Schutz vor Konkurrenz; eine Reglementierung der Berufsausübung ist grundgesetzlich nicht intendiert. ${ }^{492}$ Nach alledem und weil Planungen oder Subventionierungen

484 Für eine generelle Grundrechtsträgerschaft gemischtwirtschaftlicher Unternehmen, an denen auch Privatpersonen beteiligt sind, Jarass, in: ders./Pieroth, GG, 10. Aufl. (2009), Art. 19 Rn. 18a m.w.N.

485 Grundsätzlich für Grundrechtsfähigkeit Rüfner, in: Isensee/Kirchhof (Hrsg.), Handbuch des Staatsrechts Band V, 2. Aufl. (2000), § 116 Rn. 81.

486 Vgl. Huber, in: von Mangoldt/Klein/Starck, GG, Bd. 1, 5. Aufl. (2005), Art. 19 Abs. 3 Rn. 286 ff.

487 Manssen, in: von Mangoldt/Klein/Starck, GG, Bd. 1, 5. Aufl. (2005), Art. 12 Rn. 69.

488 BVerfGE 8, 274, 328.

489 Manssen, in: von Mangoldt/Klein/Starck, GG, Bd. 1, 5. Aufl. (2005), Art. 12 Rn. 69.

490 Breuer, in: Isensee/Kirchhof (Hrsg.), Handbuch des Staatsrechts der Bundesrepublik Deutschland, Band VI (1989), § 147 Rn. 63.

491 Breuer, a.a.O., m.w.N.

492 BVerfGE 55, 261 (269). 
ebenfalls als Eingriffe wirken können, ${ }^{493}$ wird die Wettbewerbsfreiheit unter Art. 12 Abs. 1 GG rubriziert ${ }^{494}$ bzw. nach anderer Ansicht unter Art. 2 Abs. 1 GG. ${ }^{495}$ Sie erfaßt vorzugswürdiger Auffassung zufolge die phänomenologische Zuordnung der vorgenannten Aspekte zur Freiheit wirtschaftlicher Betätigung, ohne daß ihr ein spezifisch eigenständiger materieller Gehalt zukommt. ${ }^{496}$ In allererster Linie wird dies mit berufsspezifischen Verhalten (am Markt) zusammenhängen, so daß Art. 2 Abs. 1 GG (jedenfalls) nur subsidiär zum Tragen kommt.

Das Betreiben eines Krankenhauses gilt als Beruf und unterliegt dem Schutz des Art. 12 Abs. 1 GG. 497 Abgesehen von den Freiheiten der laufenden Unternehmensführung kommen ferner die Unternehmensfreiheit, gerichtet auf die freie Gründung sowie Führung eines Unternehmens, und die davon abzugrenzende Unternehmerfreiheit der kollektiv agierenden Gesellschafter zu Tragen, ohne daß alle beruflichen Einzelheiten stets scharf voneinander abgegrenzt werden könnten. 498 Dies ist auch nicht erforderlich, soweit damit keine konkrete Zuordnung zur Berufsausübung oder -wahl verbunden ist, die für die Rechtfertigung eines Eingriffs erheblich ist. 499

Eingriffe in den Schutzbereich unterliegen der Rechtfertigung auf gesetzlicher Grundlage. Speziell für die Berufsfreiheit ergeben sich dabei besondere Anforderungen im Sinne der Stufenlehre, die im Apothekerurteil des $B V \operatorname{erf} G^{500}$ entwickelt wurde und im Bereich des Vertragsarztrechts stets besondere Bedeutung erfahren hat. ${ }^{501} \mathrm{Im}$ übrigen muß auch in diesem Zusammenhang auf den Gestaltungsspielraum des Gesetzgebers im Bereich der sozialen Sicherheit verwiesen werden, ${ }^{502}$ wobei die finanzielle Stabilität und das Funktionieren der GKV gar als überragend wichtige Güter des Gemeinwohls einzustufen sind; 503 droht diesen schwere Gefahr, sind selbst objektive Berufswahlregelungen verfassungsrechtlich gerechtfertigt.

Es verwundert nicht, daß die (individuelle) Vertragsfreiheit - zumal dann, wenn man sie unter Art. 2 Abs. 1 GG fasst - in besonderer Weise „eingeschränkt“ ist bzw. nur bedingt als Strukturprinzip jedenfalls der GKV fungiert. Dies folgt zum einen aus den

493 BVerfGE 82, 209 (223 ff.).

494 BVerfGE 46, 120 (137).

495 S. die N. bei Manssen, in: von Mangoldt/Klein/Starck, GG, Bd. 1, 5. Aufl. (2005), Art. 12 Rn. 70 (Fn. 286).

496 Vgl. auch Manssen, a.a.O., Rn. 70.

497 BVerfGE 82, 209 (223).

498 Breuer, in: Isensee/Kirchhof (Hrsg.), Handbuch des Staatsrechts der Bundesrepublik Deutschland, Band VI (1989), § 147 Rn. 60 f.

499 Dazu sogleich.

500 BVerfGE 7, $377 \mathrm{ff}$.

501 Siehe etwa Schnapp, in: ders./Wigge, Handbuch des Vertragsarztrechts, 2. Aufl. 2006, § 4 Rn. 63 ff.

502 BVerfGE 10, 354 (371); 18, 257 (273); zum Gleichheitssatz etwa BVerfGE 77, 84 (106 f.); BVerfGK 4, 356 (362 f.); zur Verfassungskontrolle im Bereich von Sozialrechtsreformen Sichert, Constitutional Review of Social Laws in Germany and its Impact on Law-Making, Zbornik Pravnog, Vol. 27 (2006), S. 725 ff. 
unter I.4.1. beschriebenen Zielkonflikten und entsprechender regulatorischer Ausgestaltung (etwa durch das [obligatorische] Kollektivvertragssystem). Zum anderen liegt es daran, daß aufgrund. doppelter Absicherung gem. $\S \S 53$ Abs. 1 S. 1 SGB X und $\S 69$ SGB V i.V.m. § 37 S. 1 SGB I selektivvertragliches Handeln, das überwiegend in Form des öffentlich-rechtlichen Vertrages erfolgt, nur insoweit zulässig ist, als Rechtsvorschriften nicht - auch nicht mittelbar - entgegenstehen. Dabei ist die (qua Auslegung zu ermittelnde) materielle Vertragsfreiheit im SGB V stärker eingeschränkt als die zunächst weithin zulässige Wahl der Handlungsform, denn die ,sozialrechtlich determinierte" Vertragsfreiheit fußt nur bedingt auf den Grundsätzen der Privatautonomie. Die insofern implizit zu ergründenden oder auch ausdrücklich eingeräumten Spielräume vgl. § 140a Abs.1 S. 1 SGB V - markieren so in verfassungsrechtlich zulässiger Weise den Rahmen für wettbewerbsorientiertes Verhalten mittels Selektivverträgen, unbeschadet der Bedeutung des $\S 69$ SGB V für die Anwendung der aus sozialrechtlicher Warte „externen“ Wettbewerbsordnung. Gleichwohl besteht nach dem vorstehend Gesagten ein Vertragstypenzwang nicht, doch der Spielraum ist tendenziell eng, soweit er nicht durch Vertragskombinationen oder ausdrücklich großzügig erweitert ist (vgl. § 73c SGB V).

Die praktische Relevanz der Berufsfreiheit für den Konkurrentenschutz im Bereich der Krankenhausversorgung ist nicht zu unterschätzen. Dies betrifft im Besonderen zwei Felder. Zum einen geht es um den Konkurrentenschutz in der Krankenhausplanung, denn die Aufnahme in den Plan führt zu den unter I.3.4. beschriebenen wettbewerbsrelevanten Folgen bzw. Vorteilen. In seinem Beschluß vom 24.1.2004, gestützt auf die Verfahrensgewährleistung des Art. 19 Abs. 4 GG und die Berufsfreiheit, Art. 12 Abs. $1 \mathrm{GG}$, hat das BVerfG festgestellt:

\begin{abstract}
„Die Aufnahme eines konkurrierenden Bewerbers in den Krankenhausplan schränkt die beruflichen Betätigungsmöglichkeiten für das nicht aufgenommene Krankenhaus ein“. ... „Die besondere Grundrechtsbetroffenheit... macht es erforderlich, ... hiergegen zeitnahen Rechtsschutz zu eröffnen.“ 504 „Effektiver Rechtsschutz ist [daher] nur gewährleistet, wenn dem übergangenen Krankenhaus zeitnah die Möglichkeit der Drittanfechtung eingeräumt wird“. 505
\end{abstract}

Die Entscheidung, der eine sog. „offensive“ bzw. „aktive“ Konkurrentenklage zugrunde lag, die sich dadurch auszeichnet, daß die Begünstigung eines Bewerbers unmittelbar das Zurückweisen des Mitbewerbers zur Folge hat, markiert den neu justierten Ausgangspunkt zugunsten des Instituts der krankenhausrechtlichen Konkurrentenklage. 506 Auf der Ebene der Fachgerichtsbarkeit mündete die Entwicklung zuletzt in ein Urteil des $B V \operatorname{erw} G^{507}$, welches ungeachtet des eher atypischen Sachverhalts ${ }^{508}$ (Plan-

504 BVerfGK 2, 223 (229).

505 BVerfGK 2, 223 (230).

506 Lenz, Staatliche Planung von Krankenhäusern, in: ders./Dettling/Kieser, Krankenhausrecht (2007), Rn. 20 ff.; Quaas, in: ders./Zuck, Medizinrecht (2008), S. 652 ff.

507 Urt. v. 25.9.2008, Az. 3 C 35/07, GesR 1/2009, S. 27 ff.

508 Vgl. die Anm. von Bracher, DVBl. 2009, S. 49. 
aufnahme eines Bewerbers ohne Auswahlentscheidung gegenüber bereits aufgenommenen Plankrankenhäusern) weitergehende Grundsatzerwägungen angestellt hat. Diese nicht unumstrittenen 509 - Hinweise sollen erst im Zusammenhang mit dem Konkurrenzverhalten der Wettbewerber näher betrachtet werden. 510

Ein gutes halbes Jahr nach der vorgenannten verfassungsgerichtlichen Entscheidung folgte ein weiteres wegweisendes Urteil auf dieser Linie. Gegenstand war die mit der angefochtenen Entscheidung des $B S G$ erfolgte Versagung des Rechtsschutzes eines niedergelassenen Arztes gegen die Ermächtigung eines Krankenhausarztes, sofern nicht ausnahmsweise Willkürentscheidungen vorlägen. ${ }^{511}$ Das $B V e r f G$ widersprach der Beurteilung durch das $B S G$ :

„Eine defensive Konkurrentenklage ausschließlich bei besonders schweren materiellen Mängeln der Begründetheit einer angefochtenen Ermächtigungsentscheidung zuzulassen, wird Bedeutung und Tragweite der Berufsfreiheit nicht gerecht.“ 512 ... „Eine Wettbewerbsveränderung durch Einzelakt, die erhebliche Konkurrenznachteile zur Folge hat, kann [aber] das Grundrecht der Berufsfreiheit beeinträchtigen, wenn sie im Zusammenhang mit staatlicher Planung und der Verteilung staatlicher Mittel steht.“ 513

Dieser Befund wirft Fragen auch zur verfassungsrechtlichen Beurteilung der Öffnung der Krankenhäuser für ambulante Leistungen gem. § 116b Abs. 2 SGB V im Hinblick auf die Berufsfreiheit der niedergelassenen Vertragsärzte auf. 514

\subsubsection{Grundrechtlicher Maßstab für selektivvertragliches Kontrahieren}

Soweit das Konzept der Selektivverträge vordringt und sich moderne Versorgungsformen zunehmend als ,neue Formen der Regelversorgung“ entwickeln, stellt sich aus verfassungsrechtlicher Sicht die Frage, ob und inwieweit Teilhabeansprüche entstehen, sofern Vertragspartnerschaften für die Ausübung der Berufsfreiheit unabdingbar sind. Unter den Grundbedingungen des Wettbewerbs erlangt insbesondere der grundrechtlich determinierte Konkurrentenschutz mit Blick auf mögliche Rechtsansprüche zum Abschluß von selektiven Versorgungsverträgen Bedeutung. Unbeschadet des weit reichenden Ausschlusses des nationalen Wettbewerbs- und Kartellrechts sind hier verfassungsrechtliche Maßstäbe bestimmend, die nach rechtsstaatlichen Grundsätzen selbstverständlich auch durch $\S 69 \mathrm{SGB} V \mathrm{n}$ nicht eingeengt werden dürfen.

In dem den direktem Beteiligungswettbewerb prägenden Vertragsverhältnis zwischen Krankenkassen und Leistungserbringern kommen die Berufs(ausübungs)freiheit,

509 Bracher, a.a.O.; DVB1. 2009, S. 49.

510 S. unter II.4.1.

511 Vgl. die Darstellung nach BVerfG, Kammer, Beschl. v. 17.8.2004, NZS 2005, S. 144 f.

512 BVerfG, a.a.O., S. 145.

513 BVerfG, a.a.O., S. 146.

514 Siehe dazu Vollmöller, Die Vereinbarkeit der Öffnung der Krankenhäuser für ambulante Leistungen (§116b II SGB V) mit der Berufsfreiheit der niedergelassenen Vertragsärzte (Art. 12 I GG), NZS 2006, S 572 ff., der von einer verhältnismäßiger Konkretisierung der Grenzen zwischen ambulanter und stationärer Krankenhausversorgung ausgeht, m.N. zur Gegenansicht in Fn. 4. 
Art. 12 Abs. 1 GG, sowie der Gleichheitssatz, Art. 3 Abs. 1 GG, besonders zum Tragen. Auch ohne daß die Wirkungsrichtung der Grundrechte in einen Beteiligungsanspruch verwandelt würde, vermittelt der Grundrechtsschutz eine teilhabespezifische Korrekturfunktion, wenn anderenfalls die Verwirklichung der Freiheiten effektiv kaum mehr möglich wäre. Die Krankenkassen als Körperschaften des öffentlichen Rechts bleiben auch beim selektiven Vertragsschluß an diese Maßstäbe gebunden. Chancengleichheit muß auch deshalb herrschen, weil die Beteiligung der Leistungserbringer die notwendige Möglichkeit bietet, finanzielle Nachteile wie vormals durch eingriffsrelevante Kürzung der Vergütung zur Anschubfinanzierung zugunsten der an der IV teilhabenden Marktteilnehmer auszugleichen. ${ }^{515}$ Eine Differenzierung zwischen verschiedenen Leistungserbringern bedarf sachlicher Gründe. Bedarfsgesichtspunkte dürfen dabei in Ansehung der Freiwilligkeit der Teilnahme der Versicherten keine Rolle spielen. 516

Gerade dann, wenn sich neue Versorgungsformen nachhaltig etablieren sollten - gewissermaßen zur neuen Regelversorgung werden -, müssen Neubewerbern Auswahlchancen und schließlich Teilhaberechte eingeräumt werden, sofern mit der Teilnahme ein wesentlicher Teil der Berufsausübung verbunden oder diese überhaupt erst ermöglicht wird. Die Auswahlentscheidung darf nicht zu quasi neuen Zulassungsschranken führen. ${ }^{517}$ Sozialrechtlich ist der Vielfalt der Leistungserbringer Rechnung zu tragen, Art. 2 Abs. 3 S. 1 SGB V.

\subsubsection{Eigentumsgarantie}

Als Substrat wettbewerbsorientierten Verhaltens dient das Eigentum, sowohl in Form des Sacheigentums als auch der übrigen vermögenswerten Rechte, die unter dem Schutz der Eigentumsgarantie nach Art. 14 Abs. 1 GG stehen. In seiner Gesamtheit spricht nichts dagegen, den eingerichteten und ausgeübten Gewerbebetrieb als eigentumsfähige Position anzuerkennen, 518 auch wenn die verfassungsgerichtliche Rechtsprechung diese Ausprägung mit Blick auf Art. 14 GG nicht näher konturiert hat und in der Regel kaum erkennbar ist, inwiefern ein solcher Schutz, der nur als Bestandsschutz wirken kann, über den Schutz der eigentumsfähigen Teilpositionen hinauszugehen vermag. Auch insofern ,ist der öffentlich-rechtliche Status als Plankrankenhaus mangels (privatem) Vermögenswert nicht eigentumsfähig.“ 519

515 Bohle, in: ders., Vertragsgestaltung in der Integrierten Versorgung, S. 9 (36).

516 A.a.O., S. 37.

517 A.a.O.

518 Vgl. Jarass, in: ders./Pieroth, GG, 10 Aufl. (2009), Art. 14 Rn. 10.

519 Quaas, in: Wenzel (Hrsg.), Handbuch des Fachanwalts Medizinrecht (2007), Kap. 12, S. 1117 Rn. 8. 


\subsection{Wettbewerbsrechtlicher Rahmen}

Im zunehmend wettbewerbsorientierten Gesundheitswesen mit partiell marktförmigen Strukturen nimmt die Problematik normativer Absicherung der Freiheit des Wettbewerbs und seines Schutzes vor Beeinträchtigungen stetig zu. Der maßgebliche wettbewerbsrechtliche Rahmen soll nachfolgend in groben Zügen betrachtet werden. Spezifisch verhaltensbezogene Mechanismen zur Steuerung und Begrenzung der Tätigkeit der Akteure im Wettbewerb erschließen sich im Zusammenhang mit den wettbewerblichen Beziehungen der Beteiligten (II.).

Die Ausgangsfrage lautet, ob und inwieweit Wettbewerbs- und Kartellrecht, die eine „Gesamtordnung des Wettbewerbs“ bilden, 520 im Bereich der GKV mit Blick auf § 69 SGB V überhaupt Anwendung finden, d.h. etwa selektives Kontrahieren, Kooperationen der Leistungserbringer und Netzwerke untereinander sowie den „Fusionswettbewerb“ der Krankenhäuser und Krankenkassen steuern. Vorfrage der Beschäftigung mit den Grundzügen des Wettbewerbsrechts ist mithin die nach dessen Anwendbarkeit im Bereich der sozialversicherungsrechtlich determinierten „Beziehungen der Krankenkassen zu den Leistungserbringern“, welche ggf. auch auf das Verhältnis der Leistungserbringer untereinander ausstrahlt.

\subsubsection{Allgemeine Grundsätze der Anwendbarkeit des Wettbewerbsrechts, v.a.} im Verhältnis der Krankenkassen zu den Leistungserbringern

Der nach dem GKV-WSG 521 durch das GKV-OrgWG m.W.v. 1.1.2009 erneut geänderte $§ 69$ SGB V bestimmt für das Verhältnis der Krankenkassen zu den Krankenhäusern gem. Abs. 1 S. 2:

\footnotetext{
„Die Rechtsbeziehungen der Krankenkassen und ihrer Verbände zu den Krankenhäusern und ihren Verbänden werden abschließend in diesem Kapitel, in den $\S \S 63,64$ und in dem Krankenhausfinanzierungsgesetz, dem Krankenhausentgeltgesetz sowie den hiernach erlassenen Rechtsverordnungen geregelt."
}

Danach ist die Anwendung auch des Wettbewerbs- und Kartellrechts mindestens in den vorgenannten Rechtsbeziehungen und vorbehaltlich des Abs. 2 ausgeschlossen. Das europäische Recht allerdings ist davon nicht erfaßt: Im Zeichen des Anwendungsvorrangs des Gemeinschaftsrechts vermag § 69 SGB V das europäische Wettbewerbsrecht nicht auszuschließen; das $B S G$ hat darauf ausdrücklich hingewiesen. ${ }^{522}$ Seit der auch systematischen Neufassung des $\S 69$ SGB V durch das GKV-OrgWG indes ist nunmehr

520 Köhler, in: Hefermehl/Köhler/Bornkamm, Wettbewerbsrecht, 25. Aufl. 2007, UWG Einl. Rn. 6.11.

521 Wesentlich weitergehend noch die Stellungnahme des Bundesrates (Anlage 2 zur BT-Drucks. 16/3950, S. 15) die neben der entsprechenden Anwendung des BGB auch die des UWG und des GWB (ohne Beschränkung) vorsah.

522 BSGE 89, 25 (34); Kingreen, Wettbewerbliche Aspekte des GKV-Modernisierungsgesetzes, MedR 2004, S. 188 (192); grundlegend EuGH, Urt. v. 15.7.1964, Rs. 6/64 (Costa/E.N.E.L.), Slg. 1964, 1251 (1269 f.). 
eindeutig, daß sich Abs. 2 der Vorschrift, der die entsprechende Geltung einzelner Vorschriften des GWB anordnet, auch für Verträge unter Beteiligung der Krankenhäuser gilt. ${ }^{523}$ Der erste Halbsatz der Vorschrift lautet:

„Die $\S 19$ bis 21 des Gesetzes gegen Wettbewerbsbeschränkungen gelten für die in Absatz 1 genannten Rechtsbeziehungen entsprechend“.

$\S 19$ bis 21 GWB normieren Verbotstatbestände hinsichtlich des Mißbrauchs einer marktbeherrschenden Stellung sowie des wettbewerbsbeschränkenden Verhaltens. Strittiger Beurteilung allerdings unterliegt, welche Folgen Verstöße gegen die Verbotsnorm zeitigen. Dies gilt sowohl im Hinblick auf kartellbehördliche Maßnahmen (vgl. § 32 GWB i.V.m. § 19 GWB) als auch betreffend Unterlassungs- und Schadensersatzansprüche (vgl. § 33 GWB). Im Hinblick auf Normbefehl und Durchsetzung der Verbote sprechen gute Gründe für eine teleologische Extension zugunsten der Anwendung auch der sanktionsrechtlichen Vorschriften, zumal dann, wenn man den gesetzgeberischen Hinweis darauf, die Vorschriften träfen ,in der Rechtsfolge“ auch die Krankenkassen,524 weitergehend interpretiert als allein im Sinne einer Geltungsvermittlung der Verbote auch für die Kassen. ${ }^{525}$ Die in Anknüpfung an den Verbotstabestand wesentliche Rechtsfolge aber, d.h. die eines Verstoßes gegen die $\S \S 19-21$ GWB, regelt auch das SGB V nicht abschließend; der Verweis auf den allgemeinen öffentlich-rechtlichen Unterlassungsanspruch ${ }^{526}$ bliebe hinter einer durch Analogie zu schließenden effektiven Abwehr gerade drohender Zuwiderhandlungen (vgl. § 33 Abs. 1 S. 2 GWB) zurück, die unter Vermeidung einer Materienspaltung nach Tatbestand und Folge gerade ,gewährleistet" 527, daß Kassen ihre Markmacht nicht missbrauchen und nicht diskriminieren.

Die (entsprechende) Anwendung des verbotstatbestandlich gefassten § 1 GWB hingegen kommt nach dem Gesetzeswortlaut und im Zeichen des insofern restriktiven Berichts des Ausschusses für Gesundheit 528 nicht in Betracht. ${ }^{529}$ Das BKartA hat dieses Fehlen erneut deutlich kritisiert und auf den Widerspruch hingewiesen, wenn den Kassen kartellwidriges Verhalten untersagt, indes „die Ursache, die ihnen ein solches Ver-

523 Hier waren allerdings grundsätzlich weniger Anwendungsfälle als im Verhältnis zu anderen Leistungserbringern denkbar, gleichwohl etwa im Hinblick auf Verträge nach § 116b SGB V. Im übrigen begegnete eine Benachteiligung der Krankenhäuser im Wettbewerbsschutz grundsätzlichen Bedenken. Es lag nahe, hier mindestens vom Fehlen entsprechender Vorstellungen des Gesetzgebers auszugehen, der die Änderung des $\S 69$ S. 2 SGB V ebenso in letzter Minute eingefügt weitere neue Fragen aufgeworfen hat; vgl. aber Becker/Kingreen, in: dies., SGB V (2008), § 69 Rn. 42.

524 Vgl. auch den Bericht des AfG, BT-Drs. 16/4247, S. 35.

525 S. bereits Sichert, Neue Versorgungsformen und Rabattregelungen für ausländische Leistungserbringer, EuR, Beiheft 2/2007, S. 101 (120), s. auch Engelmann (2009), jurisPK-SGB V, § 69 Rn. $114 \mathrm{ff}$.

526 Becker/Kingreen, in: dies., SGB V, 2008, § 69 Rn. 43.

527 BT-Drs. 16/4247, S. 35.

528 BT-Drucks. 16/4247, S. 35.

529 Sichert, Neue Versorgungsformen und Rabattregelungen für ausländische Leistungserbringer, EuR, Beiheft 2/2007, S. 101 (120). 
halten ermöglicht - nämlich die gesetzliche Ausnahme vom Kartellverbot - nicht beseitigt wird". 530

Ebenfalls im Zuge der Reform durch das GKV-OrgWG sind mittels positiver Antwort nunmehr „Zweifel beseitigt, ob die Vorschriften, die die Pflicht zur Ausschreibung öffentlicher Aufträge und die konkrete Ausgestaltung dieser Verpflichtung regeln, auch auf Versorgungsverträge anwenden sind. "531 Wenn $§ 69$ auch ausweislich der Gesetzesbegründung die „unmittelbare Geltung der materiellen Vergabevorschriften“ anordnet, vermag sich die Einschränkung des Satzes 1 Hs. 2 a.E., „,soweit die dort genannten Voraussetzungen erfüllt sind“, jedenfalls nicht mehr auf die Frage des personellen Anwendungsbereichs, d.h. die Frage nach der Eigenschaft der Kassen als öffentlichen Auftraggebern 532 i.S.d. $\S 98 \mathrm{GWB}$ zu beziehen. ${ }^{533}$ Dies gilt streng genommen nur für Aufträge oberhalb der Schwellenwerte, die allein $\S 69$ SGB V mit Blick auf das GWB betrifft. Indes machen Überlegungen in Richtung divergierender Interpretation bereits mit Blick auf $\S 22$ SVHV $^{534}$ wenig Sinn. Angesichts dessen werden sich die Streitfragen nunmehr v.a. darauf kaprizieren, ob es sich bei den jeweiligen Verträgen tatsächlich um öffentliche Aufträge handelt. Darauf ist noch gesondert einzugehen. 535

Gem. § 69 Abs. 2 S. 2 SGB V finden weder Wettbewerbsrecht noch Kartellrecht Anwendung, soweit es um Kollektivverträge geht. Auch soweit Leistungserbringer gegenüber den Krankenkassen einen Anspruch auf Abschluss eines Vertrages haben, kommt die Pflicht zur Ausschreibung unter Beachtung des Vergaberechts nicht in Betracht. Schließlich ist bei einer Überprüfung der Verträge gem. § 69 Abs. 2 S. 3 SGB V die besondere Aufgabenstellung der GKV zu beachten, etwa im Hinblick auf das Erfordernis flächendenkender Versorgungsstrukturen.536 Es verwundert nicht, daß dieses Aufeinandertreffen von Wettbewerbsschutz und Regulierung zur Sicherstellung der Versorgung erneut auf den Widerspruch des BKartA stößt, das in der Implementierung dieses „systemfremden Prüfkriteriums“ einen „deutlichen Systembruch“ sieht und auch einen Verstoß gegen EU-Richtlinien nicht für ausgeschlossen hält. ${ }^{537}$ Unter Steuerungsgesichtspunkten werden der Widerspruch zur ordnungspolitischen Grundkonzeption des deutschen Kartellrechts und die Herausbildung eines sektorspezifischen Vergaberechtsprinzips gegen die marktöffnende Tendenz des Vergaberechts angeprangert.

530 Stellungnahme vom 8. Oktober 2008 zu BT-Drs. 16/9559.

531 BT-Drucks. 16/10609, S. 65.

532 Dazu Sichert, Gemeinschaftsrechtliche Vorgaben für Integrierte Versorgung, GuS 2007, S. 28 (31); nunmehr, positiv zur Auftraggebereigenschaft, Schlussanträge des GA Mazák v.16.12.2008, Rs. C300/07 sowie jüngst EuGH, Urt. v. 11.4.1009, C-300/07 (Oymanns), NJW 2009, S. 2427 ff.

533 Vgl. aber die umfassende Prüfung bei Engelmann, (2009), juris-PK, § 69 Rn. 188 ff.

534 S. 4.4.5., die Vorschrift nimmt allerdings „Verträge, die der Erbringung gesetzlicher oder satzungsmäßiger Versicherungsleistungen dienen", von der Ausschreibung aus.

535 S. u. 4.4.5. sowie II. 3.1.7.

536 BT-Drucks. 16/10609, S. $66 \mathrm{f}$.

537 Stellungnahme vom 8. Oktober 2008 zu BT-Drs. 16/9559. 
Ferner ist die Bedeutung des $\S 69$ SGB V, der sich nicht auf eine Rechtswegzuweisung reduzieren lässt, auch im Hinblick auf das Verhältnis der Leistungserbringer untereinander zu erfassen: Jedenfalls haben $B S G^{538}$ und $B G H$ dem $\S 69$ SGB V eine materielle Ausschlußwirkung nationaler wettbewerbsrechtlicher Ansprüche attestiert, die sich ,auch auf die Beziehungen von Leistungserbringern untereinander“ erstreckt, ,soweit es um Handlungen in Erfüllung des öffentlich-rechtlichen Versorgungsauftrags der Krankenkassen geht“.539 Eine Doppelqualifikation jedenfalls kommt grundsätzlich nicht - nicht mehr - in Betracht. ${ }^{540}$ Inwiefern aber das Verhältnis in den krankenversicherungsrechtlichen Bereich hineinreicht, der zum Ausschluß des Wettbewerbsrechts führt, ist mitunter problematisch zu bestimmen und reflektiert zugleich die schwierige Grenzziehung zwischen reguliertem und „,freiem“" Markt.

Wichtige Beispiele 541 und zunächst nicht unumstritten waren Entscheidungen zur Krankenhaus-Fusionskontrolle, die v.a. wegen analytischer Betrachtungen des für die Untersuchung zentralen Krankenhausmarktes bedeutsam sind. Im Ergebnis haben sowohl das Bundeskartellamt (BKartA) ${ }^{542}$ als auch - jeweils bestätigend - OLG Düssel$\operatorname{dorf}(2007)^{543}$ und $B G H(2008)^{544}$ geurteilt, daß $§ 69$ SGB V das Wettbewerbsrecht weder im Verhältnis der Krankenhäuser untereinander noch im Hinblick auf deren Verhältnis zu den Patienten ausschließt.

Vor diesem Hintergrund sind nachfolgend die Strukturkomponenten des deutschen und europäischen Wettbewerbsrechts zu beleuchten.

538 BSGE 89, 24 (33).

539 BGH Urt. v. 23.2.2006, GRUR 2006, S. 517 ff. = NJW RR 2006, S. 1046 ff.

540 BGH Urt. v. 23.2.2006, GRUR 2006, S. 517 (519) = NJW RR 2006, S. 1046 (1047 f).

541 Weitere Entscheidungen allerdings dokumentieren eine in Grund- und Detailfragen durchaus divergierende Rechtspraxis, z.B. hinsichtlich der von Internetapotheken oft geübten Praxis, bei Abgabe verschreibungspflichtiger Medikamente Gutscheine für ein zweites Erwerbsgeschäft über rezeptfreie Apothekenartikel auszugeben. Obwohl sich die umfangreiche obergerichtliche Rspr. „lediglich“ nicht darüber einig war, ob dies gegen $\S 7$ HWG bzw. § 78 AMG i.V.m. dem UWG (§§ 3, 4 Nr. 11 UWG) verstößt (dagegen etwa OLG Naumburg vom 26.8.2005, GRUR-RR 2006, S. 336339; dafür $O L G$ Köln, Beschl. v. 20.9.2005 [GRUR 2006, S. 88], gestützt auf §§ 3, 4 Nr. 11 UWG; ebenso LG Hamburg Urt. v. 17.8.2006, PharmR 2006, S. 477), urteilte das LG Osnabrück, das UWG sei gar nicht anwendbar, eine Prüfung durch $\S 69$ SGB V ausgeschlossen, Urt. v. 18.9.2006, AZ.: 18 O 487/06, www.landgericht-osnabrueck.niedersachen.de.

542 Vgl. Beschl. v. 23.März 2005, B 10 - 109/04, Öffentliche Version, abrufbar unter www.bundeskartellamt.de (Archiv, Fusionskontrollentscheidungen 2005). Näher dazu unten II. 1.1.1., 1.1.2. sowie 4.5.

543 Beschl. v. 16.1.2008, KVR 26/07, NZS 2008, S. 653.

544 Beschl. vom 11.4.2007, Az.: VI-Kart 6/05 (V); zuvor war eine Ministererlaubnis, § 42 GWB, am 22.5.2006 abgelehnt worden, vgl. unter www.bmwi.de (Pressemitteilungen, 22.5.2006). 


\subsubsection{Zusammenwirken von nationalem und europäischem Recht}

Die „wettbewerbliche Gesamtordnung“ besteht aus eng verzahnten Teilordnungen sowohl des nationalen und europäischen Rechts ${ }^{545}$ als auch der Bereiche Lauterkeitsrecht (Wettbewerbsrecht im engeren Sinne) und Kartellrecht. Daß § 69 SGB V die Anwendbarkeit des europäischen Rechts nicht auszuschließen vermag, wurde soeben dargelegt. Kartell- und Wettbewerbsrecht ergänzen einander im Hinblick auf „übereinstimmende Schutzzwecke, nämlich den Schutz des Wettbewerbs im Allgemeininteresse und im Interesse der Marktteilnehmer".546 Während das Kartellrecht die Freiheit des Wettbewerbs gegen Beschränkungen schützt, ist Schutzrichtung des Wettbewerbsrechts die Abwehr unlauterer Wettbewerbsmethoden. ${ }^{547}$ Gem. Art. 3 der VO Nr. 1/2003 wenden auch die Wettbewerbsbehörden und Gerichte der Mitgliedstaaten Art. 81 f. EGV an; klarstellenden Charakter ${ }^{548}$ entfaltet insofern $\S 22$ GWB. Das Europarecht erlangt zentrale Bedeutung im Hinblick auf das Kartellverbot (Art. 81 Abs. 1 EGV) und das Verbots des Mißbrauchs einer marktbeherrschenden Stellung (Art. 82 EGV). Dabei sind Europäisches und Deutsches Kartellrecht (GWB) sowohl materiell als auch organisatorisch miteinander verschränkt. 549 Das kartellwidrige Verhalten kann - v.a. im Bereich der individuellen Behinderung von Mitbewerbern - muß aber nicht notwendigerweise zugleich einen Verstoß gegen das Gesetz gegen den unlauteren Wettbewerb (UWG) begründen; dies ist im Einzelfall anhand der Normen des UWG und deren Wertung zu ermitteln. ${ }^{550}$ Die vorstehend beschriebenen Regime zum Schutze des Wettbewerbs flankieren das Vergaberecht, das vor allem im GWB auf der Grundlage europäischen Richtlinienrechts verankert ist, ${ }^{551}$ und das Beihilferecht gem. Art. 87 ff. EGV. Betont man die marktfreiheitliche Komponente, entfalten neben der national verfassungsrechtlich verankerten Berufsfreiheit auch die Grundfreiheiten ${ }^{552}$ des gemeinsamen Marktes Schutzfunktion, sowohl mit Blick auf das Leistungs- als auch - zunehmend - für das Leistungserbringungsrecht.

545 Vgl. unter Bezug auf (Vorlage-) Verfahren allein in den Monaten März bis Juni 2007 Sichert, Die Rolle des europäischen Rechts für die Zukunft des deutschen Gesundheitswesens, Die BKK 2007, S. $396 \mathrm{ff}$.

546 Köhler, in: Hefermehl/Köhler/Bornkamm, Wettbewerbsrecht, 25. Aufl. 2007, UWG Einl. Rn. 6.11.

547 A.a.O.

548 Loewenheim, in: ders./Meessen/Riesenkampff, Kartellrecht, Bd. 2, 2006, § 22 Rn. 1.

549 Frenz, Handbuch Europarecht, Band 2, Europäisches Kartellrecht (2006), Rn. 146 ff., 168 ff.

550 Köhler, in: Hefermehl/Köhler/Bornkamm, Wettbewerbsrecht, 25. Aufl. 2007, UWG Einl. Rn. 6.14. ff.

551 RL 2004/18/EG m. Änd.

552 V.a. die Freiheit des Warenverkehrs, Art. 28. 30 EGV und die Dienstleistungsfreiheit, Art. 49 f. EGV. 


\subsubsection{Wettbewerbsrecht}

\subsubsection{Gesetz gegen den unlauteren Wettbewerb (UWG) und} Heilmittelwerbegesetz (HWG)

Auf nationaler Ebene schützt das UWG die Lauterkeit des Wettbewerbs zugunsten der Mitbewerber, Verbraucher und sonstigen Marktteilnehmer, § 1 S. 1 UWG. Eine mit Blick auf die $\S \S 19-21$ GWB vergleichbar entsprechende Anwendbarkeit für das Verhältnis der Krankenkassen zu den Leistungserbringern ordnet § 69 SGB V nicht an.

Dem generalklauselartigen Verbot unlauteren Wettbewerbs nach $\S 3$ UWG folgen konkrete Beispielsvorschriften nach $\S$ 4-7 UWG sowie Rechtsfolgenbestimmungen im Hinblick auf Beseitigung und Unterlassung sowie Schadensersatz und Gewinnabschöpfung, $\S \S 8-10$ UWG.

Von Bedeutung im Gesundheitswesen sind insbesondere die unzulässige Beeinträchtigung der Entscheidungsfreiheit ( $\$ 4 \mathrm{Nr}$. 1 UWG) durch Gesundheitswerbung 553 sowie das Verbot der Angstwerbung ( $§ 4$ Nr. 2 UWG), das Handeln entgegen einer gesetzlichen Vorschrift ( $\$ 4$ Nr. 11 UWG) betreffend die Klinikwerbung 554 i.V.m. berufsrechtlichen Vorschriften ${ }^{555}$ und das Verbot der irreführenden Werbung (§ 5 UWG), etwa durch Irreführung über die geschäftlichen Verhältnisse einer Klinik 556

Im Bereich der Heilmittelwerbung kommt überdies das Gesetz über die Werbung auf dem Gebiete des Heilwesens (HWG) zum Tragen. Es enthält werberechtliche Spezialtatbestände, etwa zur Irreführung ( $33 \mathrm{HWG)} \mathrm{oder} \mathrm{Angstwerbung} \mathrm{(} 7$ HWG). Tatbestandskonkurrenz zwischen HWG und UWG ist weithin möglich. 557 Soweit dies nicht der Fall ist und das HWG weitergehende Tatbestände enthält, ist meist der Rechtsbruchtatbestand nach $\S 4$ Nr. 11 UWG erfüllt. ${ }^{558}$ Dieser Gleichlauf hat Bedeutung für die vom UWG bestimmten Rechtsfolgen, während das HWG Verstöße gegen $§ 3$ nach $\S 14$ strafrechtlich und sonstige Verstöße als Ordnungswidrigkeiten ahndet.

\subsubsection{EG-Beihilfenrecht}

$\mathrm{Zu}$ den wettbewerbsrechtlichen Regelungen des Gemeinschaftsrechts gehört neben kartell- und vergaberechtlichen Bestimmungen auch das Beihilfenrecht, Art. $87 \mathrm{ff}$. EGV. Mit Blick auf die Finanzierungsseite der Krankenhäuser steht das Verbot unzulässiger Beihilfen gem. Art. 87 Abs. 1 EGV im Mittelpunkt. Nach dieser Vorschrift sind,

553 Köhler, in: Hefermeh1/Köhler/Bornkamm, Wettbewerbsrecht, 25. Aufl. 2007, § 4 UWG Rn. 1.166.

554 Köhler, a.a.O., § 4 UWG Rn. 11.114.

555 Dazu 4.4.6.

556 Bornkamm, in: Hefermehl/Köhler/Bornkamm, Wettbewerbsrecht, 25. Aufl. 2007, § 5 UWG Rn. $5.28 \mathrm{ff}$.

557 Bülow, in: ders./Ring, Heilmittelwerbegesetz, Kommentar, 2005, Einführung Rn. 26 f.

558 Bülow, A.a.O., Rn. 28 ff. 
„,soweit ... nicht etwas anderes bestimmt ist, ... staatliche oder aus staatlichen Mitteln gewährte Beihilfen gleich welcher Art, die durch die Begünstigung bestimmter Unternehmen ... den Wettbewerb verfälschen oder zu verfälschen drohen, mit dem Gemeinsamen Markt unvereinbar, soweit sie den Handel zwischen den Mitgliedstaaten beeinträchtigen.“

Da Krankenhäuser im Sinne des funktionalen Unternehmensbegriffs 559 ungeachtet der Trägerschaft und Organisationsform wirtschaftlich tätig sind, 560 ist mit Blick auf die Konkurrenzsituation zu Krankenhäusern in privater Trägerschaft zu prüfen, ob ein für öffentliche Häuser z.T. gewährter Verlustausgleich den Beihilfetatbestand erfüllt. Mangels Selektivität bzw. Begünstigung bestimmter Unternehmen wird demgegenüber die Investitionsfinanzierung nach dem KHG als Teil des dualistischen Finanzierungssystems trotz landesweiter Unterschiede von vornherein nicht als Verstoß gegen das Beihilfeverbot gewertet bzw. in ähnlicher Weise problematisiert. 561

Die Erfüllung des Beihilfetatbestandes durch Verlustausgleich hätte u.a. die Pflicht zur Notifizierung nach Art. 88 Abs. 3 EGV zur Folge, deren Missachtung bereits zur formellen Rechtswidrigkeit führte. In Anbetracht gemeinwirtschaftlicher Verpflichtungen der Krankenhäuser und dafür empfangener Gegenleistungen aber liegt bereits tatbestandlich keine Beihilfe vor, wenn nach der insoweit gebotenen Übertragung der Kriterien aus der Entscheidung des EuGH in Sachen Altmark Trans 562 auf den Krankenhaussektor 563

- das begünstigte Unternehmen tatsächlich mit der Erfüllung gemeinschaftlicher Aufgaben betraut und das Pflichtenpaket klar definiert worden ist;

- die Ausgleichsparameter zuvor objektiv und transparent aufgestellt worden sind;

- der Ausgleich keine Überkompensation der Kosten unter Berücksichtigung der Einnahmen und eines angemessenen Gewinns darstellt, und

- die Kosten eines durchschnittlich gut geführten Unternehmens nicht überschritten werden, soweit die Höhe des Ausgleichs nicht im Zusammenhang mit einem Verfahren zur Vergabe öffentlicher Aufträge zu ermitteln ist.

559 EuGH, Urt. v. 23.4.1991, Rs. C-41/90 (Höfner und Elser), Slg. 1991, I-1979, Rn. 21; s. ferner EuGH, Urt. v. 22.1.2002, Rs. C-218/90 (CISAL), Slg. 2002, I-691, Rn. 22.

560 Daran ist auch mit Blick auf die differenzierende Rechtsprechung des EuGH zur Unterscheidung zwischen wirtschaftlicher und sozialer Tätigkeit nicht zu zweifeln, vgl. Becker, EU-Beihilferecht und soziale Dienstleistungen, NZS 2007, S. 169 (174). Für Einzelheiten sei auf die Problematik der Unternehmenseigenschaft der Krankenkassen, vgl. auch II. 3.1.5.

561 Vgl. auch Vollmöller, Krankenhausfinanzierung und EG-Beihilferecht, in: Bauer u.a. (Hrsg.), FS für Reiner Schmidt (2006), S. 205 (214). Anders offenbar Schwintowski, in: Bruckenberger/Klaue/Schwintowski, Krankenhausmärkte zwischen Regulierung und Wettbewerb, 2006, S. 194, der offenbar Defizitausgleich und „Ausgleichszahlungen ... (z.B. nach § 4 KHG für die Investitionen)" gleichsetzt.

562 EuGH, Urt. v. 24.7.2003, Rs. C-280/00, Slg. 2003, I-7747.; eingehend unter 4.4.4.2.

563 Vollmöller, Krankenhausfinanzierung und EG-Beihilferecht, in: Bauer u.a. (Hrsg.), FS für Reiner Schmidt (2006), S. 205 (209 ff.); Becker, EU-Beihilferecht und soziale Dienstleistungen, NZS 2007, S. 169 (174); vgl. ferner bereits Cremer, Krankenhausfinanzierung im europarechtlichen Kontext, in: Ministerium für Arbeit, Gesundheit und Soziales des Landes Nordrhein-Westfalen (Hrsg.), Krankenhausrecht: Herausforderungen und Chancen (2005), S. 23 (35 f.). 
Sind diese Kriterien hingegen nicht erfüllt und die von Art. 87 EGV geforderte Wettbewerbsverfälschung 564 sowie eine Beeinträchtigung des innergemeinschaftlichen Handels gegeben, ${ }^{565}$ erlaubt die Prüfungssystematik eine Rechtfertigung 566 gem. Art. 86 Abs. 2 EGV, 567 auch wenn mit Blick auf die Kriterien in Sachen Altmark-Trans betont wird, auf die Ausnahmevorschrift des Art. 86 Abs. 2 EGV käme es in Anbetracht der insoweit parallelen Voraussetzungen nicht mehr an. ${ }^{568}$ Die Wettbewerbsregeln gelten danach für Unternehmen, die

„mit Dienstleistungen von allgemeinem wirtschaftlichen Interesse betraut sind ... soweit die Anwendung dieser Vorschriften nicht die Erfüllung der ihnen übertragenen Aufgabe rechtlich oder tatsächlich verhindert.“569

Um diesbezüglich Kriterien für den Bereich der Daseinsvorsorge zu fassen, hat die Kommission mit dem sog. „Monti-Paket“, das im wesentlichen aus einem Gemeinschaftsrahmen und einer Entscheidung besteht, entsprechende Vorgaben gemacht. 570

\subsubsection{Kartellrecht}

\subsubsection{Nationales Kartellrecht}

Grundlage des nationalen Kartellrechts ist das Gesetz gegen Wettbewerbsbeschränkungen (GWB) zum Schutze des Wettbewerbs vor Beschränkungen.

Regelungen über wettbewerbsbeschränkende Vereinbarungen, Beschlüsse und abgestimmte Verhaltensweisen enthalten $\S \S 1-3$ GWB. Obwohl auch wettbewerbsbeschränkende Vereinbarungen seitens der Krankenkassen prüfwürdig wären, etwa im Hinblick

564 Auf deren Spürbarkeit es nicht ankommt.

565 Dazu in vorliegendem Zusammenhang Vollmöller, Krankenhausfinanzierung und EG-Beihilferecht, in: Bauer u.a. (Hrsg.), FS für Reiner Schmidt, S. 205 (212 f.).

566 Im Ergebnis ohne Bedeutung ist eine subtile Unterscheidung zwischen der Einordnung als (Bereichs-) Ausnahme oder Rechtfertigung. Beide Kategorien werden tw. synonym verwendet, vgl. Bauer, Rechtssicherheit bei der Finanzierung gemeinwirtschaftlicher Leistungen?, EuZW 2006, S. 7 (8): „Rechtfertigung nach der Bereichsausnahme“. Das lässt sich letztlich damit begründen, daß Rechtsfolge zwar das Abweichen von den Vertragsbestimmungen ist (vgl. Koenig/Kühling, in: Streinz [Hrsg.], EUG/EGV, Kommentar, 2003, Art. 86 EGV Rn. 39), Art. 86 Abs. 2 allerdings eine Reihe von Voraussetzungen aufstellt.

567 Vgl. Bauer, a.a.O., S. 9; Becker, EU-Beihilferecht und soziale Dienstleistungen, NZS 2007, S. 169 (174). Eine Ausnahme bzw. Freistellung gem. Art. 87 Abs. 2 und Abs. 3 EGV kommt vorliegend nicht in Betracht.

568 Vollmöller, Krankenhausfinanzierung und EG-Beihilferecht, in: Bauer u.a. (Hrsg.), FS für Reiner Schmidt, S. 205 (213); siehe demgegenüber Becker, EU-Beihilferecht und soziale Dienstleistungen, NZS 2007, S. 169 (174 m.w.N.), der jedoch konzediert, daß „Unterschiede ... kaum ersichtlich“ sind (S. 175).

569 Art. 86 Abs. 2 S. 2 allerdings bestimmt: Die Entwicklung des Handelsverkehrs darf nicht in einem Ausmaß beeinträchtigt werden, das dem Interesse der Gemeinschaft zuwiderläuft.

570 Näher unter 4.5.2. 
auf gemeinsame Ausschreibungen von Rabattverträgen, 571 erlaubt $§ 69$ S. 2 SGB V die (entsprechende) Anwendung des $\S 1$ GWB nicht. Wettbewerbsbeschränkende Vereinbarungen der Krankenhäuser hingegen sind uneingeschränkt an $\S 1 \mathrm{GWB}$ zu messen. Die Nichtigkeit wettbewerbswidrigen Verhaltens folgt aus $\S 134$ BGB. ${ }^{572}$

Dasselbe gilt für Verstöße gegen das Verbot der marktbeherrschenden Stellung gem. $\S 19$ Abs. $1 \mathrm{GWB}^{573}$ sowie für den Behinderungstatbestand nach $\S 20$ Abs. $1 \mathrm{GWB}^{574}$ Gem. $\S 69$ S. 2 SGB V finden die $\S \S 19-21$ GWB auf die Beziehungen der Krankenkassen zu den Leistungserbringern einschließlich der Krankenhäuser entsprechende Anwendung. 575

Die Kartellbehörde 576 kann Unternehmen oder Vereinigungen von Unternehmen verpflichten, eine Zuwiderhandlung gegen Vorschriften des GWB oder gegen Art. 81 und Art. $82 \mathrm{EGV}$ abzustellen ( $§ 32 \mathrm{GWB}$ ) und ggf. einstweilige Maßnahmen treffen (§ 32a GWB). Entsprechende Verstöße sowie solche gegen eine Verfügung der Kartellbehörden verpflichten den wettbewerbswidrig Handelnden überdies gegenüber dem Betroffenen zur Beseitigung und bei Wiederholung zur Unterlassung, § 33 Abs. 1 S. 1 GWB. ${ }^{577}$ Aus $\S 33$ Abs. 3 GWB folgt eine Schadensersatzpflicht, und § 34 GWB ermöglicht der Kartellbehörde, durch Verstöße erlangte wirtschaftlich Vorteile abzuschöpfen. 578

Die Zusammenschlusskontrolle nach $\S \S 35$ ff. GWB erlangt für die Fusion von Krankenhäusern besondere Bedeutung. Das $O L G$ Düsseldorf 579 und nachfolgend der $B G H^{580}$ haben entschieden, daß ein Ausschluß der Kontrollvorschriften gem. § 69 SGB $\mathrm{V}$ nicht in Betracht komme. Die Schwellenwerte für den Geltungsbereich der Zusammenschlußkontrolle liegen deutlich unter denen des Gemeinschaftsrechts. ${ }^{581} \mathrm{Gem}$. § 39 GWB sind Zusammenschlüsse (vgl. § $37 \mathrm{GWB}$ ) anzumelden. Ist von dem Zusammenschluß zu erwarten, daß er eine marktbeherrschende Stellung begründet oder verstärkt, ist dieser gem. § 36 Abs. 1 GWB vom BKartA zu untersagen, wobei die Mitteilung über den Eintritt in das Hauptprüfungsverfahren Voraussetzung ist, § 40 Abs. 1

571 Näher Sichert, Neue Versorgungsformen und Rabattregelungen für ausländische Leistungserbringer, EuR, Beiheft 2/2007, S. 101 (117 ff.)

572 Nordemann, in: Loewenheim/Meessen/Riesenkampff, Kartellrecht, Bd. 2, 2006, Vor §§ 1-3 Rn. 3.

573 Götting, a.a.O., § 19 Rn. 101.

574 Loewenheim, a.a.O., § 20 Rn. 104.

575 Vgl. unter I.4.4.1.

576 Zum Verfahren I.4.5.2.1.

577 Der Unterlassungsanspruch besteht bereits dann, wenn eine Zuwiderhandlung droht, § 33 Abs. 1 S. 2 GWB.

578 Siehe zur Anwendung der Sanktionsvorschriften ferner 4.4.1.

579 Beschl. vom 11.4.2007, Az.: VI-Kart 6/05 (V).

580 BGH, Beschl. v. 16.1.2008, KVR 26/07, NZS 2008, S. 653 (654).

$581 \S 35$ Abs. 1 GWB. Anwendungsvoraussetzung ist, daß im letzten Geschäftsjahr vor dem Zusammenschluß die beteiligten Unternehmen insgesamt weltweit Umsatzerlöse von mehr als $€ 500$ Mio. und mindestens ein beteiligtes Unternehmen im Inland Umsatzerlöse von mehr al € 25 Mio. erzielt haben. 
S. 1 GWB. Wann eine Marktbeherrschung mit Blick auf den sachlich und räumlich relevanten Markt vorliegt, ergibt sich aus $\S 19$ Abs. 2 GWB.

\subsubsection{Europäisches Kartellrecht}

Das Verhalten der Akteure bzw. Marktteilnehmer ist ferner am Maßstab des europäischen Kartellrechts zu messen, Art. 81 und Art. 82 EGV. 582 Der Kontrolle unterliegt vor allem das Verhalten der mit den Krankenhäusern selektiv kontrahierenden Krankenkassen, soweit letztere als Unternehmen zu qualifizieren sind, aber auch das Wettbewerbsverhältnis der Krankenhäuser untereinander, speziell im Verhältnis der Binnenkooperation um gemeinschaftliche Verbünde, die ihrerseits mit den Krankenkassen kooperieren. Im Bereich des Kartellverbots sind - die Unternehmenseigenschaft der Krankenkassen unterstellt - vor allem Einkaufskartelle i.S.d. Art. 81 Abs. 1 lit. a EGV bzgl. integrierter Versorgungsmodelle denkbar, des gleichen im Hinblick auf ebenfalls wettbewerbswidrige Absprachen der Krankenhäuser über Preise. Die für die Spürbarkeit der Wettbewerbsbeschränkung 583 maßgebliche Schwelle nach den korrespondierenden de minimis-Regeln der Kommission liegt bei einem noch tolerablen Marktanteil von 10\% bei horizontalen Vereinbarungen. 584 Die überdies erforderliche Eignung einer Beeinträchtigung des zwischenstaatlichen, weit zu verstehenden Handels 585 kann auch dann anzunehmen sein, wenn ein unmittelbarer Bezug, etwa im Sinne im- oder exportgegenständlicher Waren, fehlt. Bei größeren Mitgliedstaaten genügt, daß sich die Absprache nur auf einen wesentlichen Teil des Hoheitsgebietes auswirkt; 586 im übrigen könnte der Bezug durch Kooperationsstrukturen im Grenzgebiet, etwa „grenzüberschreitende“ Versorgungsverträge zur medizinischen Rehabilitation i.V.m. § 140e SGB V 587 gegeben sein. Sowohl auf Seiten der Krankenkassen als auch der Krankenhäuser als Leistungserbringer wäre darüber hinaus ein Ausbeutungsmißbrauch in Form des Konditionenmißbrauchs, Art. 82 lit. c EGV denkbar. Die Nichtigkeit ggf. wettbewerbswidriger Vereinbarungen oder Beschlüsse von Unternehmensvereinigungen folgt aus Art. 81

582 Zum Verfahrensrecht unter vorrangiger Beteiligung der Kommission 4.5.2.

583 Das Spürbarkeitserfordernis gilt freilich auch für die Beeinträchtigung des zwischenstaatlichen Handels, vgl. KOM, E. v. 8.9.1997, ABl. Nr. L 271/28 (Nederlandse Vereniging van Banken u.a.); EuGH, Urt. v. 17.6.1997, Rs. C-291/95 P (Ferriere Nord/Kommission), Slg. 1997, I-4411, Rn. 19.

584 Bekanntmachung der Kom über Vereinbarungen von geringer Bedeutung, die den Wettbewerb nicht spürbar beschränken, AB1. 2001, Nr. C 368, S. 13, Ziff. 7 (zur Nichtanwendbarkeit bei „Kernbeschränkungen“ Ziff. 11); s. auch Brinker, in. Schwarze, EU-Kommentar, 2. Aufl. (20099, Art. 81 EGV Rn. 44 ff.

585 Vgl. bereits EuGH, Urt. v. 31.5.1979, Rs. 22/78 (Hugin/Kommission), Slg. 1979, 1869, Rn. 17.

586 Siehe die „Leitlinien über den Begriff der Beeinträchtigung des zwischenstaatlichen Handels in den Artikeln 81 und 82 des Vertrages“, ABl. EU Nr. C 101, S. 81 (Ziff. 77 ff. [89 ff.]), vgl. ferner EuGH, Urt. v. 25.10.2001, Rs. C-475/99 (Ambulanz Glöckner), Slg. 2001, I-8089, Rn. 47 ff.

587 Sichert, Gemeinschaftsrechtliche Vorgaben für Integrierte Versorgung, G+S 2007, S. 28 ff. 
Abs. 2 EG ${ }^{588}$, diejenige mißbräuchlichen Verhaltens aus Art. 82 EG i.V.m. § 134 BGB. 589

Das europäische Fusionskontrollverfahren hat bis dato im Krankenhaussektor keine Rolle gespielt, ganz im Gegensatz zum nationalen Verfahren. Das liegt daran, daß der für die gemeinschaftsweite Bedeutung hier maßgebliche Umsatz die Schwellenwerte 590 von $€ 5$ Mrd. bzw. $€ 2,5$ Mrd. weltweit bzw. 250 Mio. gemeinschaftsweit respektive 100 Mio. in mindestens drei Mitgliedstaaten praktisch nicht überschreitet. 591

\subsubsection{Vergaberecht}

Bereits $\S 69$ Abs. 2 S. 1 HS. 2 SGB V, der wesentliche Vorschriften des GWB für entsprechend anwendbar erklärt, macht die Beschäftigung mit dem Vergaberecht erforderlich. Nach allgemeinen Grundsätzen beschaffen Öffentliche Auftraggeber Waren, Bau- und Dienstleistungen nach Maßgabe der $\S 97 \mathrm{ff}$. GWB im Wettbewerb und im Wege transparenter Vergabeverfahren, § 97 Abs. 1 GWB. $\S \S 97$ ff. GWB sind Ausdruck der Umsetzung des zuvorderst sekundärrechtlich geprägten Vergaberechts der EG. Die aufgrund der Art. 47 Abs. 2, Art. 55 und Art. 95 EGV ergangene neue Vergaberichtlinie 2004/18/EG wurde insbesondere durch die Änderung 592 der Vergabeverordnung (VgV, vgl. § 97 Abs. $6 \mathrm{GWB})$ und der hier maßgeblichen Verdingungsordnung für Leistungen - Teil A (VOL/A) 2006 und der Verdingungsordnung für freiberufliche Leistungen (VOF) 2006 umgesetzt, wenngleich derzeit noch nicht vollständig. ${ }^{593}$ Letztere sind gem. $\S \S 4$ bzw. 5 der VgV anzuwenden, wobei gem. $\S 9$ VOF und $\S 17 \mathrm{a}$ im Abschnitt 2 der VOL/A grundsätzlich die europaweite Ausschreibung verlangen. Voraussetzung ist jeweils, daß die hier relevanten neuen Schwellenwerte der Auftragswerte von mittlerweile $€ 206.000^{594}$ erreicht werden. Sind diese nicht erfüllt, ist nach haushaltsrechtlichen Grundsätzen vorzugehen, wobei das Recht der Träger (s. § 22 Abs. 2 SVHV) mit dem Verweis ,allgemeiner Richtlinien“ auf die Verdingungsordnungen Bezug nimmt; 595 allerdings sind nach $\S 22$ Abs. 1 SVHV „Verträge, die der Erbringung

588 Bei Ausschreibungen trifft dies in der Regel zunächst nur diese selbst. Das Schicksal der Folgeverträge bleibt in der Regel (gemeinschaftsrechtlich) unberührt, vgl. Eilmansberger, in: Streinz (Hrsg.), EUV/EGV, 2003, Art. 81 EG Rn. 104.

$589 \mathrm{Zu}$ letzterem Armbrüster, in: Münchener Kommentar zum Bürgerlichen Gesetzbuch, Band 1, 5. Aufl. 2006, § 134 Rn. 37; Jung, in: Calliess/Ruffert, EUV/EG, 3. Aufl. 2007, Art. 82 EG Rn. 73.

590 Näher Art. 1 Abs. 2 und 3 der VO (EG) Nr. 139/2004 („EG-Fusionskontrollverordnung“), ABl. EU 2004 Nr. L 24, S. 1.

591 Siehe Bohle, Die Bedeutung des Kartell- und Wettbewerbsrechts bei Krankenhausfusionen, MedR 2005, S. 259 (260).

592 Vom 23. Oktober 2006, BGBl. I, S. 2334.

593 Vgl. auch die kleine Anfrage vom 30.3.2008, BT-Drucks. 16/4928.

594 Vgl. VO (EG) Nr. 1422/2007, Art. 2 Nr. 1 lit. b (ABL. Nr. L $317 / 34$ [35]) i.V.m. Art. 7 lit. b 3. Spiegelstr. RL 2004/18/EG. Vormals war ein Schwellenwert von $€ 211.000$ maßgeblich.

595 Obwohl RL der Träger gemeint sind, geht die Praxis tw. von der unmittelbaren, tw. von der mittelbaren Geltung der Verdingungsordnungen aus; hinter diesen dürfen die RL mithin nicht substantiell zurückbleiben. 
gesetzlicher oder satzungsmäßiger Versicherungsleistungen dienen“, von der Pflicht zur Ausschreibung ausgeschlossen. Dies gilt auch, soweit die Erbringung von Pflichtleistungen selektivvertraglich organisiert wird.

Bei (sog. nachrangigen) Dienstleistungen im Gesundheits- und Sozialwesen allerdings ist eine europaweite Bekanntmachung nicht erforderlich. ${ }^{596}$ Unbeschadet dessen bleiben die Anforderungen nach GWB und $\mathrm{VgV}$ unberührt. Auch insoweit müssen ein transparentes Verfahren gewährleistet sein und der Gleichbehandlungsgrundsatz beachtet werden, vgl. § 97 GWB. Dabei gilt gem. § 101 Abs. 6 S. 1 GWB der Vorrang des offenen Verfahrens. Der Auftraggeber muß schließlich die nicht berücksichtigten Bieter schriftlich spätestens 14 Tage vor dem Vertragsschluß informieren und den Namen des Bieters, dessen Angebot angenommen worden ist, ebenso mitteilen wie den Grund der vorgesehenen Nichtberücksichtigung; ein vor Ablauf der Frist oder ohne Erteilung der Information geschlossener Vertrag ist nichtig, § $13 \mathrm{VgV}$.

Nach im Ergebnis übereinstimmender Auffassung stand $\S 69$ SGB V bereits vor dessen Neufassung i.R.d. GKV-OrgWG der Anwendung des Vergaberechts im Verhältnis der Kostenträger zu den Leistungserbringern nicht prinzipiell entgegen. Dies folgt nach wie vor - auch für Auftragssummen unterhalb der Schwellenwerte - aus der europarechtlichen Prägung des nationalen Vergaberechts und der hiernach i.V.m. dem Anwendungsvorrang 597 des Gemeinschaftsrechts gebotenen gemeinschaftskonformen Auslegung. 598 Überdies wurde darauf hingewiesen, es sei nicht Intention des § 69 SGB V, diskriminierende und behindernde Verhaltensweisen dem Anwendungsbereich des GWB zu entziehen, ${ }^{599}$ sowie betont, es gehe bei § 69 SGB V um die Negation der Eigenschaft der Krankenkassen als Unternehmen i.S.d. Privatrechts, wohingegen der Status als ,öffentlicher Auftraggeber“ insoweit von § 69 SGB V nicht erfaßt sei. 600 Während die grundsätzliche Anwendbarkeit des Vergaberechts mit Blick auf § 69 SGB V bereits vor dessen Neufassung durch das GKV-OrgWG zu bejahen war, ist nunmehr

596 Bei Anwendung der VOL/A ergibt sich dies aus § 1a Ziff. 2 Abs. 2 des Abschnitts 2 i.V.m. Anhang I, Teil B Kategorie 25; anwendbar bleiben die Basisparagraphen sowie $\S \S 8 \mathrm{a}$ und 28a des zweiten Abschnitts. Bei Anwendung der VOF folgt die Ausnahme von der europaweiten Ausschreibung aus $\S 2$ Abs. 1 S. 2 i.V.m. Anhang I, Teil B, Kategorie 25; anwendbar bleiben $\S 8$ Abs. 2 und 7 und $\S$ 17. Europarechtlich sind Art. 21 i.V.m. Anhang II Teil B Kategorie 25 der RL 2004/18/EG i.V.m. den CPV Nrn. - vgl. VO (EG) Nr. 213/2008, Abl. Nr. L 74, S. 1 (293) - 85110000-3 (Dienstleistungen von Krankenhäusern und zugehörigen Einrichtungen) und 85111000-0 (Dienstleistungen von Krankenhäusern) einschlägig. Bei eindeutig grenzüberschreitendem Interesse indes sind weiterhin die fundamentalen Grundsätze der Grundfreiheiten maßgeblich, s. EuGH, Urt. v. 13.11.2007, Rs. C-507/03, Slg. I, 9777, Rn. 25 ff.

597 Siehe bereits EuGH, Urt. v. 15.7.1964, Rs. 6/64 (Costa/E.N.E.L.), Slg. 1964, 1251 (1269 f.).

598 Siehe Hesselmann/Motz, Integrierte Versorgung und Vergaberecht, MedR 2005, S. 498 (499 m.w.N.); Kaltenborn, Vergaberechtliche Strukturen im Recht der Gesetzlichen Krankenversicherung, VSSR 2006, S. 357 (361f. m.w.N.). Vgl. ferner BSGE 89, 25 (34); Kingreen, Wettbewerbliche Aspekte des GKV-Modernisierungsgesetzes, MedR 2004, S. 188 (192).

599 Hesselmann/Motz, a.a.O., S. 499.

600 Kingreen, Wettbewerbliche Aspekte des GKV-Modernisierungsgesetzes, MedR 2004, S. 188 (192); BT-Drs. 14/1245 S. 48. 
auch geklärt, daß das Vergaberecht für Selektivverträge der Krankenkassen Anwendung findet, ${ }^{601}$ so daß sich - die Auftraggebereigenschaft auch unterhalb der Schwellenwerte ggf. unterstellt ${ }^{602}-($,,nur“) noch die Frage stellt, ob bzw. unter welchen Voraussetzungen diese als öffentliche Aufträge i.S.d. § 99 GWB zu qualifizieren sind. 603

\subsubsection{Berufsrecht}

Für die wettbewerbsorientierte Beurteilung der „Zuweisungssteuerung“ der Patienten entfaltet auch das ärztliche Berufsrecht Grenzfunktion, insbesondere bei der Einweisungssteuerung der Krankenhäuser durch (finanzielle) Anreize für ärztliches Verhalten auf beiden Seiten. Darüber hinaus bildet das Berufsrecht - mindestens mittelbar - den Maßstab für das Werbeverhalten der Kliniken; in dieser Konstellation stellen sich oftmals Fragen der Zurechnung, da das ärztliche Berufsrecht vom Leitbild des freien Berufs, nicht der gewerblichen Tätigkeit der Krankenhäuser ausgeht.

Grundlage des ärztlichen Berufsrechts sind die Berufsordnungen der Ärztekammern als autonomes Satzungsrecht in berufsständischer Selbstverwaltung. ${ }^{604}$ Sie ergehen auf der Grundlage der Kammergesetze der Länder; in den Kammern besteht Zwangsmitgliedschaft. Die Berufsordnungen konkretisieren die „Generalpflichtklauseln“ in den Kammergesetzen. ${ }^{605}$ Als maßgebliche Empfehlung zu deren allgemeiner Konkretisierung fungiert die Musterberufsordnung der Bundesärztekammer für die deutschen Ärztinnen und Ärzte, aktuell überwiegend in der Beschlussfassung des 107. Deutschen Ärztetages in Bremen (2004). ${ }^{606}$ Die Landesberufsordnungen sind weitgehend inhaltsgleich mit der Musterberufsordnung. 607

Normhierarchisch rangiert das Berufsrecht als Satzungsrecht unter dem formellen Gesetzesrecht. Als Verstoß gegen eine „Marktverhaltensregelung“ vermag ein Verstoß gegen die Berufsordnung jedoch zugleich einen solchen gegen das UWG zu begründen, der durch Schadensersatz- und Unterlassungsansprüche geahndet werden kann. ${ }^{608}$ Überdies sind Sanktionen im berufsgerichtlichen Verfahren möglich, wobei der Entzug

601 S. oben I.4.4.1.

602 Soweit wegen $\S 22$ Abs. 1 SVHV überhaupt relevant, s. oben 4.4.1.

603 S. II. 2.2.

604 Ratzel/Lippert, Kommentar zur Musterberufsordnung der Deutschen Ärzte (MBO), 4. Aufl. 2006, Einleitung; Hoppe/Schirmer, in: Wenzel (Hrsg.), Handbuch des Fachanwalts Medizinrecht (2007), Kap. 9, S. 821 Rn. 21 ff.

605 Hoppe/Schirmer, a.a.O., Rn. 43.

606 Geändert durch den Beschluss des Vorstands der Bundesärztekammer in der Sitzung vom 24.11.2006 (§ 18 Absatz. 1). Vgl. zur Überweisung eines Antrags auf „Aufnahme eines ethischen Kodex zur Ablehnung der Zusammenarbeit mit der Tabakindustrie in die (Muster-)Berufsordnung“" an den Vorstand der Bundesärztekammer Ziff. 25 des Beschlußprotokolls des 110. Deutschen Ärztetages vom 15.-18. Mai 2007 in Münster, abrufbar unter www.bundesaerztekammer.de.

607 Vgl. die Berufsordnung für die Ärzte Bayerns v. 6. August 2007, neu bekannt gemacht am 1. August 2005, Bayerisches Ärzteblatt 9/2005, S. 623, sowie am 28. April 2007, Bayerisches Ärzteblatt 7-8/2007, S. 422.

608 Näher unter II.1.1.5. 
der Approbation nur im Wege verwaltungsbehördlicher Entscheidung statthaft ist. ${ }^{609}$ Bei alledem ist darauf zu achten, daß berufsrechtliche Einschränkungen ärztlicher Tätigkeiten, insbesondere Werbeverbote, an der Verfassung, d.h. vor allem an der Berufsfreiheit (Abs. 12 Abs. $1 \mathrm{GG}$ ) zu messen sind. 610

Im Lichte jüngerer wettbewerbsorientierter Entwicklungen hat das Berufsrecht in Form der MBO für Ärzte mit $\S 18$ (Berufliche Kooperation) und $\S \S 23 \mathrm{a} f f$. (Ärztegesellschaften und sonstige Kooperationen und Partnerschaften) reagiert. Mit Blick auf die Krankenhäuser indes sollen zwei Regelungen aus den Bereichen berufliche Kommunikation und Wahrung der ärztlichen Unabhängigkeit herausgegriffen werden:

$\S 27$ Abs. 3 MBO: Berufswidrige Werbung ist dem Arzt untersagt. Berufswidrig ist insbesondere eine anpreisende, irreführende oder vergleichenden Werbung. Werbeverbote aufgrund anderer gesetzlicher Bestimmungen bleiben unberührt.

§ $31 \mathrm{MBO}$ : Ärztinnen und Ärzten ist es nicht gestattet, für die Zuweisung von Patientinnen und Patienten oder Untersuchungsmaterial ein Entgelt oder anderen Vorteile sich versprechen oder gewähren zu lassen oder selbst zu versprechen oder zu gewähren.

\subsection{Kontrollbehörden}

Die zur Kontrolle der gesetzeskonformen Wettbewerbstätigkeit der Krankenhäuser und Krankenkassen sind das BKartA und die Europäische Kommission sowie die Aufsichtsbehörden der Krankenkassen. Soweit letztere nicht bereits als Vergabeprüfstellen oder -kammern agieren, sind sie aufsichtsrechtlich für die Einhaltung der Vorschriften der solidarischen Wettbewerbsordnung zuständig..

\subsubsection{Nationale Kartellbehörden}

Nationale Kartellbehörden sind das BKartA, das Bundesministerium für Wirtschaft und Technologie und die nach Landesrecht zuständigen obersten Landesbehörden, § 48 Abs. 1 GWB. Soweit das GWB eine Zuständigkeit nicht einer bestimmten Kartellbehörde zuweist, nimmt das BKartA die gem. GWB der Kartellbehörde übertragenen Aufgaben und Befugnisse wahr, ,wenn die Wirkung des wettbewerbsbeschränkenden oder diskriminierenden Verhaltens oder einer Wettbewerbsregel über das Gebiet eines Landes hinausreicht", § 48 Abs. 2 S. 1 GWB. In den übrigen Fällen nimmt die nach Landesrecht zuständige oberste Landesbehörde diese Aufgaben und Zuständigkeiten wahr, $\S 48$ Abs. 2 S. 2 GWB. Im Rahmen ihrer Zuständigkeit (vgl. §§ 48 f. GWB) sind das BKartA und die obersten Landesbehörden die für die Anwendung der Art. 81 und 82 EGV in Einzelfällen zuständigen Wettbewerbsbehörden (Art. 5 VO (EG) $\mathrm{Nr}$.

609 Hoppe/Schirmer, in: Wenzel (Hrsg.), Handbuch des Fachanwalts Medizinrecht (2007), Kap. 9, S. 825 Rn. 38.

610 Näher Hoppe/Schirmer, a.a.O., S. 833 Rn. 75 ff. 
1/2003), 611 welche gem. Art. 35 der VO zu bestimmen sind. Dies und Einzelheiten zum Vollzug des europäischen Rechts bestimmt $\S 50 \mathrm{GWB}$, während $\S 50 \mathrm{a}$ GWB v.a. den Informationsaustausch im Netzwerk der europäischen Wettbewerbsbehörden regelt.

Das BKartA ist selbständige Bundesoberbehörde im Geschäftsbereich des Bundesministeriums für Wirtschaft und Technologie, § 51 Abs. 1 GWB. Die Entscheidungen werden von Beschlußabteilungen getroffen, § 51 Abs. 2 GWB. Nach maßgeblichem Organisationsplan vom Februar 2009 ist die dritte 3. Beschlußabteilung (B. 3) für Gesundheit einschließlich der Krankenhäuser zuständig. ${ }^{612}$. Das BKartA fällt Entscheidungen in den vorstehend beschriebenen Bereichen des Kartellverfahrens und der Fusionskontrolle sowie im Vergaberecht. Besondere Aufmerksamkeit haben in jüngerer Zeit die Verfahren (dazu $§ 40 \mathrm{GWB}$ ) um Zusammenschlüsse der Krankenhäuser erregt. Bis zum Jahre 2006 hat das BKartA insgesamt 50 Fusionen im Krankenhaussektor geprüft. 613 Im März 2005 wurde erstmals ein Zusammenschluß untersagt ${ }^{614}$ und im Laufe des Jahres ein weiterer; eine Freigabe erfolgte unter Auflagen. ${ }^{615}$

\subsubsection{Europäische Kommission}

\subsubsection{Kartellverfahren}

Zentrale Befugnisse zur Anwendung der in den Art. 81 und Art. 82 EGV niedergelegten Wettbewerbsregeln sind der Kommission zugewiesen und seit dem 1.Mai 2004 in der VO Nr. 1/2003 auf der Grundlage des Art. 83 EGV verankert (s. Art. 4 VO Nr. 1/2003). Sowohl die KOM als auch die Wettbewerbsbehörden der Mitgliedstaaten und deren Gerichte sind für die Anwendung der Art. 81 und 82 EGV zuständig, Art. 4-6 VO Nr. 1/2003. Fragen der Zusammenarbeit einschließlich der einheitlichen Anwendung des gemeinschaftsrechtlichen Wettbewerbsrechts regeln Art. 11-16 VO Nr. 1/2003. Innerhalb der Kommission ist die Generaldirektion Wettbewerb mit der Anwendung der Art. 81 und 82 EGV befaßt. 616

Die Kommission wird auf eine Beschwerde hin 617 oder von Amts wegen tätig und kann bei einer festgestellten Zuwiderhandlung gegen Art. 81 oder 82 EGV die beteiligten Unternehmen und Unternehmensvereinigungen verpflichten, die Zuwiderhandlung

611 AB1. EG 2003, Nr. L 1.

612 Vgl. unter www.bundeskartellamt.de (wir über uns, Struktur).

613 Böge, Der Markt für Krankenhausfusionen aus Sicht des Bundeskartellamts, in: Klauber/Robra/Schellschmidt (Hrsg.), Krankenhaus-Report 2006 (2007), S. 35 ff.

614 A.a.O.

615 Bangard, Fusionskontrolle bei Krankenhäusern, Symposium „Krankenhaus und Recht“, Präsentation, S. 9.

616 Vgl. auch Art. 217 Abs. 2 und Art. 218 Abs. 2 EGV sowie Art. 21 der Geschäftsordnung der Kommission i.d.F. des Beschlusses 2005/961/EG/Euratom, ABl. 2005 Nr. L 347/83.

617 Beschwerdebefugt sind natürliche und juristische Personen, die ein berechtigtes Interesse darlegen, sowie die Mitgliedstaaten, Art. 7 Abs. 2 VO Nr. 1/2003. 
abzustellen. ${ }^{618}$ Sie kann Abhilfemaßnahmen verhaltensorientierter oder struktureller Art vorschreiben; diese müssen verhältnismäßig und zur Abstellung der Zuwiderhandlung erforderlich sein. ${ }^{619}$ Sofern von gleicher Wirksamkeit und weniger belastend, sind verhaltensorientierte vor strukturellen Maßnahmen zu verhängen. ${ }^{620}$ In dringenden Fällen kann die Kommission bei ernsten und irreparablen Schäden einstweilige Maßnahmen treffen. ${ }^{621}$ Die Kommission verfügt über weit reichende Ermittlungsbefugnisse, Art. 17-22 VO Nr. 1/2003. Sowohl zur Durchsetzung dieser Befugnisse als auch zur Sanktion für eine Zuwiderhandlung gegen Art. 81 oder Art. 82 EGV kann sie Geldbußen verhängen. ${ }^{622}$ Die Nichtbefolgung von Entscheidungen, Aufforderungen oder Verpflichtungszusagen ${ }^{623}$ kann Zwangsgelder nach sich ziehen. 624

\subsubsection{Prüfung der Vereinbarkeit von Beihilfen mit Gemeinschaftsrecht}

Der Kommission ist ferner aufgegeben, die Vereinbarkeit von Beihilfen i.S.d. Art. 87 Abs. 1 EGV mit dem Gemeinsamen Markt zu überprüfen; ihr obliegt die Beihilfeaufsicht. Neben der besonderen Rolle der Kommission bestehen Zuständigkeiten des Rates. 625

Das je nach Charakter bzw. Einführungsstadium der Beihilfen diversifizierte Verfahren der Beihilfeaufsicht ist in Art. 88 EGV geregelt und wird in wesentlichen Teilen sekundärrechtlich durch die VO (EG) Nr. 659/1999 (auf der Grundlage des Art. 89 EGV) des Rates und die Durchführungsverordnung (EG) Nr. 794/2004 der Kommission flankiert.

Jede beabsichtige Einführung oder Umgestaltung einer Beihilfe ist der Kommission gem. Art. 88 Abs. 3 EGV zu melden. Für in diesem Sinne anmeldepflichtige „neue Beihilfen" 626 ist ein Genehmigungsverfahren gem. 2-9 VO (EG) Nr. 659/1999 durchzuführen. ${ }^{627}$ Dies erfolgt zunächst in Form eines vorläufigen Prüfverfahrens, an dessen Ende die Kommission entscheiden kann, daß keine Beihilfe vorliegt, keine Einwände zu erheben oder das förmliche Prüfverfahren zu eröffnen, Art. 4 Abs. 2-4 VO Nr. 659/1999. Für die Einleitung des vorläufigen Verfahrens sowie für die Anmeldung bestimmter Änderungen bestehender Beihilfen im vereinfachten Verfahren sind besondere Regelungen gem. Art. 3 bzw. 4 der VO Nr. 794/2004 maßgeblich. Zum Ende des förmlichen Verfahrens kann die Kommission entscheiden, daß die angemeldete Maßnahme keine

618 Art. 7 Abs. 1 S. 1 VO Nr. 1/2003.

619 Art. 7 Abs. 1 S. 2 VO Nr. 1/2003.

620 Art. 7 Abs. 1 S. 3 VO Nr. 1/2003.

621 Art. 8 VO Nr. 1/2003.

622 Art. 23 VO Nr. 1/2003.

623 Art. 9 VO Nr. 1/2003.

624 Art. 24 VO Nr. 1/2003.

625 Art. 88 Abs. 2 UAbs. 3, Art. 89 EGV.

626 Art. 1 lit. c VO Nr. 659/1999.

627 Näher Frenz, Handbuch Europarecht, Band 3, Beihilfe- und Vergaberecht (2007), Rn. 1267 ff. 
Beihilfe ist oder aber als solche mit dem Gemeinsamen Markt vereinbar ist, sie kann diese Positiventscheidung mit Bedingungen oder Auflagen versehen oder negativ entscheiden, daß die Beihilfe wegen Unvereinbarkeit mit dem Gemeinsamen Markt nicht eingeführt werden darf, Art. 7 Abs. 2-5 VO Nr. 659/1999. Entscheidungsempfänger sind die Mitgliedstaaten. ${ }^{628}$ Im Verfahren beteiligte oder zu beteiligende Dritte ${ }^{629}$ können gegen die Entscheidung der Kommission die Nichtigkeitsklage in Form der Individualklage (Art. 230 Abs. 4 EGV) erheben, wenn sie sich mit den Begünstigten im Wettbewerb befanden und eine spürbare Beeinträchtigung ihrer wettbewerblichen Situation geltend machen können. ${ }^{630}$ Die mit den Empfängern von Beihilfen konkurrierenden Krankenhausunternehmen einschließlich zukünftiger und potentieller Wettbewerber, insbesondere Kliniken in privater Trägerschaft, sind Beteiligte des Kontrollverfahrens nach Art. 88 Abs. 2 EGV und können zulässiger Weise auch eine Untätigkeitsklage dagegen erheben, daß die Kommission auf eine eingereichte Beschwerde hin keine Entscheidung über die Unvereinbarkeit der Beihilfe fällt. ${ }^{631} \mathrm{Im}$ Fall der Asklepios Kliniken $\mathrm{GmbH} /$ Kommission und Deutschland hat das EuG indes entschieden, in Anbetracht der Komplexität der Angelegenheit - die Beschwerde bezieht sich auf mehr als 700 öffentlicher Häuser ohne individuelle Bezeichnung und beanstandet sowohl Ausgleichszahlungen für Betriebsverluste als auch Garantien - sei eine Dauer der Vorprüfung einer Beschwerde von 12 Monaten nicht unangemessen. 632

Die VO Nr. 659/1999 sieht drei Verfahren zur Kontrolle von Beihilfen vor, welche der Kommission überantwortet sind. ${ }^{633}$ Sie betreffen das Verfahren bei rechtswidrigen Beihilfen, ${ }^{634}$ bei der missbräuchlichen Verwendung von Beihilfen ${ }^{635}$ sowie die fortwährende Überprüfung bestehender Beihilferegelungen. ${ }^{636}$

\subsubsection{Durchführung vergaberechtlicher Bestimmungen}

Schließlich sind der Kommission im Bereich des Vergaberechts spezifische Befugnisse zur Durchführung des sekundärrechtlich determinierten Vergaberechts zugewiesen. Sie ist Adressat zahlreicher Berichtspflichten, etwa der Mitgliedstaaten betreffend die Verzeichnisse der Einrichtungen und Kategorien von Einrichtungen des öffentlichen Rechts als „öffentliche Auftraggeber“ 637, oder Bekanntmachungspflichten der öffentli-

628 Art. 25 VO Nr. 659/1999.

629 Art. 1 lit. h, Art. 20 VO Nr. 659/1999.

630 Näher Burgi, in: Rengeling/Middeke/Gellermann, Handbuch des Rechtsschutzes der Europäischen Union, 2. Aufl. (2003), Rn. 68 ff. m.w.N.

631 EuGH, Urt. v. 11.7.2007, Rs. T-167/04 (Asklepios Kliniken GmbH/Kommission und Deutschland), EuZW 2007, S. $505 \mathrm{ff}$.

632 A.a.O., Rn. 89 f. (S. 510 f).

633 Näher Frenz, Handbuch Europarecht, Band 3, Beihilfe- und Vergaberecht (2007), Rn. 1328 ff.

634 Art. 10-15 VO Nr. 659/1999.

635 Art. 16 VO Nr. 659/1999.

636 Art. 17-19 VO Nr. 659/1999.

637 Art. 1 Abs. 9 UABs. 3 S. 2 RL 2004/18/EG. 
chen Auftraggeber selbst. ${ }^{638}$ Gelangt die Kommission vor Abschluß eines Vertrages zu der Auffassung, daß ein ,klarer und eindeutiger Verstoß gegen Gemeinschaftsvorschriften für die Auftragsvergabe" vorliegt, kann sie unmittelbar einschreiten und dem Auftraggeber und dem Mitgliedstaat entsprechende Mitteilung machen. ${ }^{639}$ Der Mitgliedstaat muß binnen $30 \mathrm{bzw}$. 21 Tagen die Beseitigung des Verstoßes bestätigen bzw. deren Unterlassung begründen oder über eine Aussetzung informieren. ${ }^{640}$ Dieser Korrekturmechanismus findet sich im nationalen Recht in $\S 21 \mathrm{der} V \mathrm{gV}$ wieder. Das Nachprüfungsverfahren, $\S 102 \mathrm{ff}$. GWB, bleibt unberührt.

\subsubsection{Aufsichtsbehörden der Krankenkassen}

Wie andere Versicherungsträger auch unterliegen die Krankenkassen der Aufsicht, $\S \S 87$ ff. SGB V. Diese ist Rechtsaufsicht ( 87 Abs. 1 S. 2, § 89 Abs. 1 S. 1 SGB IV), auch in Belangen der normativen Vorgaben für das Verhalten im Wettbewerb. Die Aufsichtsbehörde kann die Geschäfts- und Rechnungsführung des Versicherungsträgers prüfen, § 88 Abs. 1 SGB IV.

Die Aufsicht über bundesunmittelbare Versicherungsträger, d.h. solche, deren $\mathrm{Zu}$ ständigkeitsgebiet sich über das Gebiet eines Landes hinaus erstreckt, ${ }^{641}$ führt das BVA, § 90 Abs. 1 SGB IV. ${ }^{642}$ Erstreckt sich der Zuständigkeitsbereich der Versicherungsträger nicht über das Gebiet eines Landes hinaus, führen über diese landesunmittelbaren Versicherungsträger die für die Sozialversicherung zuständigen obersten Verwaltungsbehörden der Länder oder durch Rechtsverordnung übertragenen Behörden Aufsicht. Soweit das BVA den M-RSA durchführt und den Gesundheitsfonds verwaltet (vgl. §§ 266, 271 SGB V), ist es Verwaltungsbehörde. ${ }^{643}$

Die Landesverbände unterstehen der Aufsicht der für die Sozialversicherung zuständigen obersten Verwaltungsbehörden des Landes, in dem sie ihren Sitz haben, § 208 SGB V. Der Spitzenverband Bund untersteht der Aufsicht des BMG (§ 217d S. 1 Hs. 1 SGB V), bei Entscheidungen zum Beitrags- und Meldeverfahren gem. $\S 217 \mathrm{f}$ Abs. 3 S. 1 SGB V bzw. Empfehlungen nach S. 2 dem BMAS (§ 217d S. 1 Hs. 2 SGB V).

\section{Art. 35 RL 2004/18/EG.}

639 Art. 8 der RL 2004/18/EG, Art. 3 der RL 89/665/EWG (Sektorenrechtsmittelrichtlinie); vgl. ferner den Vorschlag zur Änderung beider Richtlinien nach KOM (2006) 195 endg.

640 Art. 8 Abs. 3 der RL 92/13/EWG, Art. 3 Abs. 3 der RL 89/665/EWG.

641 Darüber hinaus bestimmt Art. 87 Abs. 2 S. 2 GG: „Soziale Versicherungsträger, deren Zuständigkeitsbereich sich über das Gebiet eines Landes, aber nicht über mehr als drei Länder hinaus erstreckt, werden abweichend von Satz 1 als landesunmittelbare Körperschaften des öffentlichen Rechtes geführt, wenn das aufsichtsführende Land durch die beteiligten Länder bestimmt ist.“

642 Soweit ihm gem. $\S 137$ Abs. 1 S. 1 SGB V die Zulassung von DMP obliegt, es den RSA im Gesundheitsfonds durchführt ( $\$ 266$ Abs. 5 und 6 SGB) sowie den Gesundheitsfonds als Sondervermögen verwaltet (§ 271 Abs. 1 SGB V), ist es Durchführungsbehörde, vgl. Pfohl/Sichert, Der Gesundheitsfonds: Sondervermögen des Bundes oder der Krankenkassen?, NZS 2009, S. 71 ff. 
Das Aufsichtsrecht korrespondiert mit der Pflicht der Krankenkassen, auf Verlangen der Behörde alle Unterlagen vorzulegen und alle Auskünfte zu erteilen, die gefordert werden, $\S 88$ Abs. 2 SGB IV. Aufsichtsmittel sind die Beratung und - nachgeordnet die Verpflichtung des Versicherungsträgers, die Rechtsverletzung zu beheben. Bei Unanfechtbarkeit oder Anordnung der sofortigen Vollziehung kann die Verpflichtung mit den Mitteln des Verwaltungsvollstreckungsrechts durchgesetzt werden.

Eine wichtige Aufgabe kommt den Aufsichtsbehörden bei der - nun auch kassenartübergreifend zulässigen - Vereinigung der Krankenkassen zu. Sowohl für die kassenartinterne (vgl. etwa $\S 144$ Abs. 1 S. 2 SGB V) als auch die kassenartübergreifende Fusion (s. § 171a Abs. 1 S. 2 SGB V) bedarf der von den Verwaltungsräten zu fassende Beschluß der Genehmigung der vor der Vereinigung zuständigen Aufsichtsbehörden. Die Fusionskontrolle nach $\S 35$ ff. GWB kommt daneben - dies ist strittig644 - nicht in Betracht.

Ist die Leistungsfähigkeit einer Krankenkasse nicht mehr auf Dauer gesichert, wird die Krankenkasse von der Aufsichtsbehörde geschlossen (vgl. §§ 146, 153, 163, 170 SGB V).

Mit dem GKV-OrgWG wurden den Aufsichtsbehörden im Zuge der Herstellung der Insolvenzfähigkeit aller Krankenkassen (vgl. § 171b SGB V m.W.v. 1.1.2010) weitere zentrale Befugnisse eingeräumt. Gem. § 171b Abs. 3 S. 1 SGB V kann „der Antrag auf Eröffnung des Insolvenzverfahrens über das Vermögen der Krankenkassen... nur von der Aufsichtsbehörde gestellt werden. “645 Zur Vermeidung der Schließung oder Insolvenz von Krankenkassen ermöglicht $\S 172$ d Abs. 3 SGB V den Aufsichtsbehörden, auch gegen den Willen einer in ihrer Leistungsfähigkeit gefährdeten Krankenkasse die Voraussetzungen für die Vereinigung mit einer anderen zur Vereinigung bereiten Krankenkassen herbeizuführen. Nur die Aufsichtsbehörde kann ferner einen Antrag nach $\S$ 265a Abs. 1 SGB V auf Gewährung finanzieller Hilfen zur Vermeidung der Schließung oder Insolvenz einer Krankenkasse stellen (Abs. 2), die im übrigen nur gewährt werden können, wenn bereits 646 - öffentlich-rechtliche - Verträge über freiwillige finanzielle Hilfen in ausreichender Höhe nach $§ 265 b$ SGB V geschlossen worden sind. Diese Verträge sind von den zuständigen Aufsichtsbehörden zu genehmigen.

\section{S. II.5.1.3.2.}

645 „Liegen zugleich die Voraussetzungen für eine Schließung wegen auf Dauer nicht mehr gesicherter Leistungsfähigkeit vor, soll die Aufsichtsbehörde anstelle des Antrages nach Satz 1 die Krankenkassen schließen.“, § 171b Abs. 3 S. 2 SGB V m.W.v. 1.1.2010.

$646 \mathrm{Ob}$ eine gleichzeitige Vereinbarung ausreicht, lässt sich nach dem Vertragszweck des $§ 265 b$ SGB V bezweifeln; jedenfalls aber dürfte sich im Falle aktualisierter Erforderlichkeit „,freiwilliger“ Hilfen zur Einhaltung des Subsidiaritätsgrundsatzes als Antragsvoraussetzung kaum ein bereiter Vertragspartner finden. 
4.5.4. Zuständigkeiten im vergaberechtlichen Nachprüfungsverfahren gem. GWB

Die Vergabe öffentlicher Aufträge unterliegt der Nachprüfung durch Vergabekammern, unbeschadet der Prüfungsmöglichkeiten von Aufsichtsbehörden und Vergabeprüfstellen. Die Einrichtung solcher - streitschlichtend und beratend tätigen - Vergabeprüfstellen, die außerhalb des förmlichen Rechtsschutzverfahrens stehen, ist Bund und Ländern freigestellt und kann auch bei Fach- und Rechtsaufsichtsbehörden erfolgen. 647 Für den Bund wird das Bundesversicherungsamt als Vergabeprüfstelle für die Vergabe von Liefer-, Bau- und Dienstleistungen durch bundesunmittelbare Sozialversicherungsträger prüfend tätig.

Die sachliche Zuständigkeit der Vergabekammern des Bundes und der Länder folgt den Vergabeverfahren für die jeweilige Gebietskörperschaft, vgl. § 104 Abs. 1 GWB. Der Bund richtet die erforderliche Anzahl von Vergabekammern beim Bundeskartellamt ein, § 106 Abs. 1 S. 1 GWB.

647 Siehe Heuvels, in: Loewenheim/Meessen/Riesenkampff, Kartellecht, Bd. 2, GWB (2006), § 102 Rn. 20, § 103 Rn. 1. 


\section{Wettbewerb und Regulierung in der Krankenhausversorgung}

Nachfolgend gilt es Eröffnung, Ausfüllung, Gestaltung und Kontrolle wettbewerblich relevanter Verhaltensspielräume in den jeweiligen Wettbewerbsbeziehungen zu eruieren. Sie orientieren sich an den zentralen Strukturkomponenten des Versorgungsangebotes, des Absatzmarktes sowie an (verbleibenden) Optionen zur Preisgestaltung. Von besonderer Bedeutung ist dabei die Feststellung, daß die maßgeblichen Wettbewerbsparameter im Grunde multipolar erfaßt werden müssen: So ist die Schaffung von Angebotsstrukturen unter Ausschöpfung wettbewerblich relevanter Verhaltensspielräume gleichzeitig an Anreizen für das spätere Wahlverhalten der Nachfrager orientiert. Dieselben Wettbewerbsparameter sind oftmals angetan, sich in mehreren Wettbewerbsverhältnissen gleichzeitig auszuwirken. Preiswettbewerb im Selektivvertragswettbewerb beeinflusst Versorgungsangebot und Nachfrage durch die Krankenkassen ebenso wie den (Konzentrations-)Wettbewerb der Leistungserbringer sowie der Kostenträger untereinander. Damit stellt sich die Frage einer prägnanten Zuordnung. Entscheidendes Kriterium sollen weniger die für die jeweiligen Verhältnisse mittelbar zu erzielenden Effekte, z.B. besonderer Profilierung gegenüber konkurrierenden Leistungsanbietern mittels Werbung, sondern die unmittelbar gesetzten Anreizfunktionen, wie im Beispiel die Adressierung an die Patienten sein. Daß die rechtliche Kontrolle des Werbeverhaltens maßgleich durch Rechtsschutzersuchen der betroffenen Konkurrenten initiiert wird, ändert an der Zuordnung nichts.

Auf der anderen Seite lässt es sich nicht umgehen, funktionale Teilsaspekte, etwa solche der Angebotsseite, mehrfach, aber unterschiedlich prononciert zuzuordnen: So bestimmt Managed Care mittels selektiven Vertragswettbewerbs das Verhältnis zwischen den Kostenträgern und Krankenhäusern, erzeugt gleichzeitig aber einen Kooperationswettbewerb der Leistungserbringer untereinander („Vorab-Integration“) und einen „Angebotswettbewerb“ der daraufhin mit den Krankenkassen entwickleten Versorgungsmodelle zur Wahl durch die Versicherten.

In Anbetracht der weithin fehlenden direkten Verbindungen ist das Verhältnis der Kostenträger untereinander differenziert zu bestimmen. Der Fusionswettbewerb der Kassen jedenfalls fällt unter krankenhausspezifischen Gesichtspunkten weniger schwer ins Gewicht. Im übrigen betrifft etwa die Angebotsgestaltung durch Wahltarife selbstverständlich den „Kassenwettbewerb“, setzt operativ aber beim „Mitgliederwettbewerb“ an. Damit wird entbehrlich, den bereits im Verhältnis zu den Versicherten betrachteten Angebotswettbewerb nochmals unter Gesichtspunkten der Konkurrenz der Kassen untereinander gesondert zu gewichten. Interessant allerdings bleibt hier die Frage der steuernden Kontrolle des wettbewerbsorientiert forcierten Fusionsprozesses. 


\title{
1. Verbraucherorientierte Anreize der Krankenhausauswahl
}

Der Erfolg der Krankenhäuser im Wettbewerb hängt maßgeblich von der Wahl des Hauses durch den Patienten ab. Soweit im Bereich der allgemeinen Krankenhausleistungen bei (landesweit) einheitlich pauschaler Vergütung ein echter Preiswettbewerb ausscheidet, avanciert die Menge der erbrachten Krankenhausleistungen im Absatzmarkt zu einem der zentralen Wettbewerbsparameter. Auf der Basis effizienter krankenhausindividueller Versorgungsstrukturen können maßgebliche Umsatzerlöse und angesichts günstiger Kostenstrukturen Gewinne bzw. günstige Netto-Ergebnisse erzielt werden. Zugleich setzten Anforderungen in Form von Mindestmengen (vgl. § 137Abs. 3 [S. 1] Nr. 2, S. 2 SGB V) ${ }^{648}$ in gleicher Weise den Zuspruch der Patienten bzw. Versicherten voraus wie die nach außen orientierte und gleichsam als informative Werbung fungierende Berichterstattung über die Versorgungsqualität, die im Internet zugänglich ist (§ 137 Abs. 3 [S. 1] Nr. 4 SGB V).

Damit stellt sich die Frage nach der Rolle des Versicherten im Gesundheitsmarkt. In seiner Fusionskontrollentscheidung vom 11.4.2007 hat sich das OLG Düsseldorf in einer bis dahin kaum vorfindbaren Deutlichkeit mit wettbewerblich relevanten Verhaltensspielräumen der Krankenhäuser auseinandergesetzt. Die zentralen Aussagen lauten:

\begin{abstract}
„Auf dem... Markt stehen sich der Patient bzw. der einweisende Arzt als sein Disponent als Nachfrager und die Krankenhäuser als Anbieter der erforderlichen stationären Krankenhausbehandlung gegenüber...“6 649 „Der Patient hat die Möglichkeit, zwischen mehreren in Betracht kommenden Krankenhäusern zu wählen... Er kann... grundsätzlich frei auswählen, in welchem zugelassenen Plankrankenhaus er sich bei Vorliegen stationärer Behandlungsbedürftigkeit behandeln lassen will.“ 650
\end{abstract}

Allerdings ist zu konstatieren, daß das $O L G$ auf die normativen Bedingungen der Steuerung nur unvollkommen eingeht; 651 die in diesem Bereich maßgebliche Beteiligung des Arztes verweist es (allein) in den Bereich der Diganose bzw. Feststellung der Notwendigkeit der Krankenhausbehandlung. ${ }^{652}$ Vor diesem Hintergrund sind zunächst die rechtlichen Grundlagen der Position als Nachfrager näher zu betrachten:

648 „Wenn die nach Satz 1 Nr. 2 erforderliche Mindestmenge bei planbaren Leistungen voraussichtlich nicht erreicht wird, dürfen entsprechende Leistungen nicht erbracht werden“, § 137 Abs. 3 S. 2 SGB V.

649 Az. VI-Kart 6/05 (V), Kart 6/05 (V), LS. 2.

650 A.a.O., Rn. 36 (zit. nach juris).

651 A.a.O., Rn.

652 A.a.O., Rn. 78. 


\subsection{Gesetzliche Krankenversicherung}

\subsubsection{Das Recht zur Wahl des Krankenhauses}

Nach der vorstehend skizzierten Bedeutung der Rolle des Patienten bzw. Versicherten mag es verwundern, daß - anders bei der PKV653 - die gesetzgeberische Entscheidung zugunsten eines Rechts der freien Wahl des Krankenhauses keinesfalls eindeutig ausfällt, sondern - jedenfalls regelungstechnisch - eher als Ausnahme erscheint; § 39 Abs. 2 SGB V bestimmt:

„Wählen Versicherte ohne zwingenden Grund ein anderes als ein in der ärztlichen Einweisung genanntes Krankenhaus, können ihnen die Mehrkosten ganz oder teilweise auferlegt werden.“

Das Recht des Patienten zur Wahl des Krankenhauses wird danach lediglich indirekt und mit Einschränkungen verankert. „Der Grundsatz der Wahlfreiheit“, so wird mitunter gesagt, „kommt in $\S 39$ Abs. 2 nicht mehr zum Ausdruck“. 654 Dieser Befund wird dadurch verstärkt, daß die Begründung zum maßgeblichen Gesetzentwurf von einem „Gebot" für den Versicherten spricht, ,das in der ärztlichen Einweisung genannte Krankenhaus in Anspruch zu nehmen“.655 Der Sache nach aber verbleibt dem Versicherten jedenfalls ein Wahlrecht (,wählen“) mit Einschränkungen, ${ }^{656}$ und es kann an dieser Stelle dahingestellt bleiben, ob man konstatieren möchte, die Norm setzte ,gedanklich ein freies Auswahlrecht... voraus."657 Wirtschaftlich wird die Wahlfreiheit durch das Risiko bestimmt, nach dem Ermessen der Krankenkassen die Mehrkosten ganz oder teilweise tragen zu müssen, ${ }^{658}$ und der autonom gesetzte Grund der Entscheidung findet keine allgemeine Ankerkennung.

Gerade die Risikobelastung zielt auf einen - bewusst intendierten - Steuerungseffekt. Denn nach $\S 73$ Abs. 4 S. 3 SGB V sind in der Verordnung von Krankenhausbehandlung in den geeigneten Fällen auch die beiden nächsterreichbaren, für die vorgesehene Krankenhausbehandlung geeigneten Krankenhäuser anzugeben; dabei ist das Verzeichnis der Leistungen und Entgelte für die Krankenhausbehandlung in den zugelassenen Krankenhäusern im Land oder in einer Region zu berücksichtigen, § 73 Abs. 4 S. 4, $\S 39$ Abs. 3 SGB V.

Während der Gedanke der (finanziellen) Planungssicherheit damit verwirklicht wird, so erscheint die Steuerung des Zugangs jedenfalls ambivalent. Die zentralen Fragen also lauten, wem grundsätzlich die Entscheidung über das letztlich aufzusuchende Krankenhaus obliegt und ob diese nach den Regeln des Wettbewerbs, genauer denen des Marktes im Sinne der Attraktivität des Krankenhauses für den Patienten als „Nachfrager“,

\section{Dazu unten 1.2.}

654 Schmidt (2006), in: Peters (Hrsg.), Handbuch der Krankenversicherung, SGB V, § 39 Rn. 230.

655 Siehe BT-Drucks. 11/2237, S. 177.

656 Schmidt (2006), in: Peters (Hrsg.), Handbuch der Krankenversicherung, SGB V, § 39 Rn. 230.

657 Noftz (2008) in: Hauck/Noftz, SGB V, K § 39 Rn. 114.

658 Noftz, a.a.O., Rn. $114 \mathrm{f}$. 
oder nach tendenziell regulativen Vorgaben erfolgen soll. Ein Qualitätswettbewerb etwa vermag sich schwerlich frei zu entfalten, wenn der verordnende Arzt Gefahr läuft, sich gegenüber der Krankenkasse durch die Einweisung in ein entferntes oder teureres Krankenhaus unter dem Gesichtspunkt des ,sonstigen Schadens“ gem. § 106 SGB V i.V.m. $\S 48$ Abs. 1 BMV(Ä) Primärkasse und $\S 44$ Abs. 1 Arzt-/Ersatzkassenvertrag regresspflichtig zu machen. 659

Das $O L G$ Düsseldorf bewertet diese Einschränkungen nicht als materielle Belastung der Wahloptionen. Es argumentiert im Gegenteil rechtlich, daß es für den Behandlungsanspruch grundsätzlich einer Einweisung nicht bedürfe 660 und Grundlage der Krankenhausversorgung im Verhältnis zum Patienten ein zwischen diesem und dem Krankenhaus geschlossener Krankenhausaufnahme- bzw. Krankenhausbehandlungsvertrag ist, der der Privatautonomie unterliege. 661 Auch der $B G H$ urteilte, ,der gesetzlich versicherte Patient, der stationärer Behandlung bedarf, wählt als Marktteilnehmer das Krankenhaus autonom“; jedenfalls würde durch drohende Mehrkosten „seine grundsätzlich bestehende Wahlfreiheit... nicht beseitigt." 662 Es handelt sich also im Grunde um eine relative Autonomie, zumal der $B G H$ mit Blick auf die Kriterien für den Arzt zur Angabe des Krankenhauses ausführt, dies seien „Kriterien, der Patient auch bei einer völlig autonomen Auswahlentscheidung berücksichtigen würde." 663

Tendenziell schwieriger Bewertung unterliegen damit weniger die Einzelaspekte juristischer Beurteilung, sondern die rechtspraktischen Steuerungseffekte und -anreize unter Gewichtung der Wettbewerbsrelevanz. Die Feststellung etwa, der wahlbezogene Auswahlspielraum bei tendenziell nur assistierendem ärztlichen Verhalten „gilt gleichermaßen auch für Notfallpatienten“,664 trifft juristisch zwar zu, wenngleich die Marktförmigkeit des Verhaltens auf Seiten des Patienten hier deutlich im Zweifel steht.

Im Ergebnis kombiniert der Gesetzgeber Strategien zur Verfolgung mehrerer Ziele unter Einschaltung mehrerer gleichzeitig berechtigter Akteure. Dies mag zwar jedes wirtschaftliche Risiko zu Lasten einer effizienten allgemeinen Gesundheitsversorgung beim Kostenträger ausschließen, hemmt aber jedenfalls einen weiter reichenden Qualitäts- und Spezialisierungswettbewerb, da und soweit an regionale Kriterien angeknüpft und die wettbewerbliche Entwicklung über den Zuspruch der Patienten weithin eingeschränkt wird.

Von der rechtlichen Ebene zu trennen ist die Frage, inwiefern sich der Patient tatsächlich zu entscheiden vermag oder nach wettbewerbsorientierten Kriterien zu entscheiden gedenkt. Zum einen herrscht eine „Informationsasymmetrie“ vor, und selbst

659 Hencke (2004), in: Peters (Hrsg.), Handbuch der Krankenversicherung, SGB V, § 73 Rn. 31.

660 Beschl. vom 11.4.2007, Az.: VI-Kart 6/05 (V), zitiert nach juris, Rn. 36, unter Hinweis auf Genzel, in: Laufs/Uhlenbruck (Hrsg.), Handbuch des Arztrechts, 3. Aufl. (2002), § 87 Rn. 7.

661 A.a.O.

662 BGH, Beschl. v. 16.1.2008, KVR 26/07, NZS 2008, S. 653 (656).

663 A.a.O., s. 657.

664 Beschl. vom 11.4.2007, Az.: VI-Kart 6/05 (V), zitiert nach juris, Rn. 53. 
der mit den Qualitätsberichten konfrontierte Patient mag sich schwer tun, die entsprechend berichteten Ergebnisse für sich zu bewerten. Darüber hinaus vermag der durch Krankheit belastete Versicherte möglicherweise ebenso wenig rational zu entscheiden, wie die nüchterne Abwägung etwa bei sozialen Bindungen im Hinblick auf religiöse Krankenhausträger positiv eine Rolle spielt.

Alles in allem treffen danach an entscheidender Stelle der normativ zulässigen Verhaltssteuerung Option und Regulativ aufeinander. Damit kommt es zur kontrollierten Steuerung des im übrigen eingeschränkten Wahlverhaltens. Daß dies nicht von der Hand zu weisen ist, zeigt auch, daß die „freie Wahl des Krankenhauses“ als Sonderleistung einer durch eine gesetzliche Krankenkasse vermittelten privaten Zusatzversicherung ausgewiesen wird. 665

Immerhin aber hat der Versicherte auch im Rahmen der Bezeichnung bestimmter Einrichtungen in der Verordnung jedenfalls die Wahl zwischen zwei genannten. Im Sinne einer Kostenreduktion zugunsten der Versichertengemeinschaft ist die regulatorische Komponente erklärbar. Nicht zuletzt in Anbetracht der - teils verwirklichten, teils gedachten und teils jedenfalls vorausgesetzten - Anreizoptionen für wettbewerbsorientiertes Verhalten indes ist die Einschränkung des Wahlrechts wiederum Ausdruck eines unvollkommenen, aber auch eines bedingt konsequenten und teilweise wenig stringenten Wettbewerbskonzepts.

Ungeachtet dessen kann der Patient unter regulierten Marktbedingungen als Nachfragender bzw. „Nachfrager“ bezeichnet werden. Denn selbst mit Blick auf die Rolle der Krankenkassen, d.h. in Anbetracht dessen, daß „sie zwar (auch) Nachfrager von Krankenhausleistungen für gesetzlich Versicherte“ sind, ,ist es der Patient, der die Nachfrage im Einzelfall auf einen bestimmten Bedarf konkretisiert und durch Abschluss des Behandlungsvertrags eigene Leistungsansprüche für sich begründet.“666

\subsubsection{Die Ausübung des Wahlrechts}

Von besonderem Interesse erscheint danach, wie sich die Auswahl der Krankenhäuser in der Praxis darstellt. Dazu soll zunächst auf eine im vorgenannten Verfahren vor dem OLG Düsseldorf vorgelegte Studie Bezug 667 genommen werden:

„Dass entweder der Patient selbst, nach Rücksprache und Beratung mit den einweisenden Arzt, das Krankenhaus auswählt oder - vor allem bei komplizierteren Behandlungen - der einweisende Arzt für seinen Patienten die Auswahlentscheidung trifft, wird durch das Ergebnis der von der Beteiligten zu 1 vorgelegten Krankenhausstudie Forschungsgruppe Wahlen aus Februar 2006 ... weiter bekräftigt. Die Studie kommt zu dem Ergebnis, dass von den Befragten, die in den letzten fünf Jahren mindestens einen stationären Krankenhausaufenthalt hatten, $41 \%$ geantwortet haben, dass der Arzt das Krankenhaus ausgewählt habe, während 39 \% das Krankenhaus selbst ausgewählt ha-

665 Vgl. Tarife der AOK Bayern „AOK-KlinikPRIVAT/1“ und „AOK-KlinikPRIVAT/2“, www.aok.de/bay/rd/129794.htm.

666 BGH, Beschl. v. 16.1.2008, KVR 26/07, NZS 2008, S. 653 (656).

667 Krankenhausstudie Forschungsgruppe Wahlen aus Februar 2006 (Bl. 509 ff. GA); zitiert gem. OLG Düsseldorf, Beschl. vom 11.4.2007, Az.: VI-Kart 6/05 (V), wiederum zitiert nach juris, Rn. 53. 
ben (S. 13 der Studie, Bl. 523 GA). Selbst auf die Frage, wie reagiert werden würde, wenn der Arzt einen kleineren, ungefährlichen Eingriff in einer Klinik empfiehlt und eine entsprechende Einweisung in dieses Krankenhaus ausstellt, haben nur $61 \%$ der Befragten geantwortet, der Einweisung zu folgen, hingegen haben $34 \%$ erklärt, diese Entscheidung nicht automatisch zu akzeptieren (S. 8 der Studie, B1. 518 GA).“

Die Beweiskraft zugunsten eines den Wettbewerb bestimmenden Wahlrechts nach Art und Umfang erscheint danach zwar nach wie vor begrenzt. Trotz aller Einschränkungen des Wahlrechts allerdings ist jedenfalls zuzugeben, daß das Verhalten in der Rechtspraxis den vom BKartA und $O L G$ verfolgten Ansatz untermauert, demzufolge der um die stationäre Behandlung nachsuchende Patient, wenngleich meist nur zusammen mit dem einweisenden Arzt als Nachfrager bzw. Nachfragedisponent der Krankenhausleistung anzusehen ist. 668

Naturgemäß allerdings hinge die finale Beurteilung der Ausübung des Wahlrechts v.a. davon ab, inwieweit sich das Angebot als flächendeckend darstellt und ob eine Elektiv- oder Notfallbehandlung in Frage steht. Bei verbreitetem Angebot etwa von Hüftoperationen als Elektivleistungen wurde einer weiteren Studie zufolge aktives Wahlverhalten konstatiert, wenngleich durchaus in Abhängigkeit vom Alter der Patienten und der Siedlungsstruktur. ${ }^{669} \mathrm{Da}$ gerade in Kernstädten in drei Viertel der Fälle nicht das nächstgelegene Krankenhaus aufgesucht wird, zeigt zwar das Bewusstsein hinsichtlich der Wahlentscheidung, aber auch die Relativität des Kriteriums „wohnortnah“. Weiter wäre zu untersuchen, inwieweit soziale und auch Bindungen zu Kirchen als Träger eine Rolle spielen.

\subsubsection{Die Qualität der Versorgung und Qualitätsberichte als maßgebliche Faktoren der Auswahlentscheidung}

Danach gilt grundsätzlich: „Das freie Wahlrecht des Patienten ist Ausdruck wirtschaftlichen Wettbewerbs." 670 Über die maßgeblichen Parameter der patientenbezogenen wettbewerbsorientierten Verhaltenssteuerung ist damit indes noch nichts ausgesagt.

Mit Blick auf das Recht der Finanzierung wurde bereits darauf hingewiesen, daß die hoch regulierte Preisgestaltung keine Preisfreiheit ermöglicht, die das Verhalten des Patienten in gewichtiger Weise steuernd beeinflussen könnte. Dies gilt jedenfalls für den zentralen Bereich der allgemeinen Krankenhausleistungen, in dem Festpreise für zeitlich begrenzte Versorgungsleistungen je Behandlungsfall vorherrschen. 671

„Trotz der gesetzlichen Vorgaben verbleibt dem Krankenhaus aber ein wettbewerblich relevanter

Verhaltensspielraum, um bei seinem Angebot von Krankenhausdienstleistungen in Konkurrenz zu anderen Krankenhäusern in seinem Einzugsgebiet treten zu können. Der Wettbewerb der Kran-

668 A.a.O., Rn. 52.

669 Friedrich/Beivers, in: Klauber/Robra/Schellschmidt (Hrsg.), Krankenhausreport 2008/2009 (2009), s. $155 \mathrm{ff}$. (178 ff.)

670 A.a.O., Rn. 38.

671 A.a.O., Rn. 39. 
kenhäuser um den frei auswählenden Patienten findet über die Qualität der Krankenhausversorgung statt." 672

$\mathrm{Ob}$ die zuletzt genannte Feststellung - Auswahl nach der Qualität der Krankenhausversorgung - in dieser Pauschalität zutrifft, mag zumindest bezweifelt werden. Fraglich ist insbesondere, wie der Patient in Abwesenheit für ihn eindeutig nachvollziehbarer (qualitativer) Indikatoren entscheidet, sollte er diesbezüglich nicht ohnehin versucht sein, den einweisenden Arzt zu befragen bzw. ihm die Entscheidung zu überlassen. Belastbare Untersuchungen zu Präferenzen innerhalb der je nach Lebenssituation grundsätzlich anzunehmenden Motivbündel (bestehend v.a. aus der räumlichen Nähe im Sinne der Erreichbarkeit [auch für Angehörige], Einschätzungen vom Hörensagen, Bindungen z.B. im Hinblick auf freigemeinnützige Träger u.a.) und ihre wettbewerbsspezifischen Auswirkungen sind kaum bekannt (s.o.). Unbeschadet der gesetzlichen, in jüngerer Zeit verschärften Anforderungen an die Qualitätssicherung der Krankenhäuser (vgl. v.a. §§ 135a, 137 SGB V) wird der Versicherte oftmals geneigt sein, die „Qualität“ nach eigener subjektiver Einschätzung, orientiert z.B. an Modernität, Zuverlässigkeit und Freundlichkeit, selbst einzuschätzen.

Zur Sicherstellung einer objektivierten Qualitätsorientierung ist seit dem Jahre 2005 erstmals allgemein über die Qualität der Krankenhausversorgung zu berichten, § 137 Abs. 3 [S. 1] Nr. 4 SGB V. Der Bericht muß im Internet veröffentlicht werden (Nr. 4 S. 2). Bericht und Publikationsmodus wurden eingeführt, um mittels der somit herzustellenden Transparenz Arzt und Versichertem die Auswahl des Krankenhauses ,auch unter Qualitätsgesichtspunkten“ zu erleichtern. ${ }^{673}$ Auch im Zeichen tendenziell eher eingeschränkter Fähigkeiten der Versicherten, die Qualitätsberichte zu interpretieren und unbeschadet der Diskussion um die objektive Abbildung der Qualität 674 dürfen die Kassenärztlichen Vereinigungen sowie die Krankenkassen und ihre Verbände auf der Basis der Berichte auch vergleichend über Qualitätsmerkmale der Krankenhäuser informieren und Empfehlungen aussprechen ( $\$ 137$ Abs. 3 S. 4 SGB V). Maß und Inhalt werden zur Vergleichbarkeit der Berichte von der ,Vereinbarung über Inhalt und Umfang eines strukturierten Qualitätsberichts bestimmt ${ }^{675}$, dessen Anlage umfangreiche Vorgaben enthält. Im Gefolge der Änderung des $§ 92$ (Abs. 1 S. 2 Nr. 13) SGB V durch das GKV-WSG haben die Vereinbarungen nunmehr stärker verbindlichen Charakter erhalten. ${ }^{676}$

672 A.a.O., Rn. 39 f.; eigene Hervorhebung; BGH, Beschl. v. 16.1.2008, KVR 26/07, NZS 2008, S. 653 (656).

673 Hencke (2004), in: Peters (Hrsg.), Handbuch der Krankenversicherung, SGB V, § 137 Rn. 3.

674 Vgl. Drösler, Qualitätsberichte gem. § 137 SGB V und ihre Darstellung im Internet - eine vergleichende Analyse, in: Klauber/Robra/Schellschmidt, Krankenhausreport 2006 (2007), S. 207 ff.; Sachverständigenrat zur Begutachtung der Entwicklung im Gesundheitswesen, Kooperation und Verantwortung, Gutachten 2007, S. 390 ff.

675 Zuletzt geändert am 18.12.2008, BAnz. 2009, S. 565.

676 S. dazu Pinter, Qualitätssicherung im Krankenhaus nach dem GKV-WSG, KHR 2008, S. 1 (3). 
Eine gewisse Informationsasymmetrie indes wird stets bestehen bleiben. Die Monopolkommission stellt für die Jahre 2006/2007 fest:

„Die Verpflichtung zur Offenlegung bestimmter Qualitätsindikatoren entfalten ihre Wirkungen daher gegenwärtig vor allen Dingen auf professioneller Ebene. Qualitätstransparenz wird überwiegend betrieben von Profis für Profis. “677

\subsubsection{Einschränkung des Rechts der Krankenhauswahl durch „Managed Care“}

Das Wahlrecht des Versicherten unterliegt ggf. weiteren Einschränkungen, nämlich dann, wenn sich der Betroffene bspw. als Teilnehmer in ein Modell der integrierten Versorgung (vgl. § 140a Abs. 3 SGB V) oder ein Disease Management Programm (DMP, $\S \S 137$ f, g SGB V, §§ 28b ff. RSAV) eingeschrieben hat. Gem. § 140a Abs. 1 S. 4 SGB V ergeben sich das Versorgungsangebot und die Voraussetzungen seiner Inanspruchnahme aus dem Versorgungsvertrag mit Bedeutung auch für die Einschränkung der freien Wahl der Leistungserbringer. ${ }^{678}$ Mit der Einfügung des neuen $\S 11$ Abs. 4 SGB V durch das GKV-WSG erfährt das Managed Care eine besondere, nämlich grundsätzliche Anerkennung, speziell zugunsten des Patienten und zur Behebung der Schnittstellenproblematik:

„Versicherte haben Anspruch auf ein Versorgungsmanagement, insbesondere zur Lösung von Problemen beim Übergang in die verschiedenen Versorgungsbereiche.“

Die Einschränkung der Wahl des Krankenhauses im Zeichen spezifischer ManagedCare Vertragskonzepte gilt freilich zunächst nur, soweit das Versorgungskonzept reicht. Dabei dürfte die bevölkerungsbezogene und flächendeckende Vollversorgung mit Budgetverantwortung im Rahmen der IV die Beschränkung auf ein oder ggf. zwei Akutkrankenhäuser für allgemeine Krankenhausleistungen zur Folge haben. Im Rahmen des Versorgungsumfangs ist die gleichzeitige Teilnahme an beiden Versorgungsformen nicht zulässig. $679 \mathrm{Im}$ übrigen bestimmt der Vertrag neben den Modalitäten auch die Dauer der Bindung.

Die Einschränkung des Wahlrechts lässt sich zunächst damit begründen, daß die Teilnahme an besonderen Versorgungsformen freiwillig erfolgt. Die Modalitäten der Bindung folgen aus den Einschreibebedingungen wie sie sich satzungsrechtlich i.V.m. mit den entsprechenden Einzelheiten des Versorgungsvertrages zwischen Krankenkasse und Leistungserbringer ergeben. $\S 137 \mathrm{f}$ Abs. 3 S. 3 SGB V bestimmt überdies ausdrücklich, daß die Einwilligung zur Teilnahme an DMP widerrufen werden kann. Wegen der Beschränkung gesetzlicher Wahlrechte wird allerdings diskutiert, ob eine satzungsrechtliche Beschränkung insofern den Anforderungen an eine formalgesetzliche Ermächtigung genügt oder stattdessen eine materielle Regelung qua spezifischer Teil-

677 Siebzehntes Hauptgutachten, BT-Drs. 16/10140, S. 320, Ziff. 821.

678 S. auch Knittel (2004), in: Krauskopf, ,Soziale Krankenversicherung/Pflegeversicherung, § 140a Rn. 5.

679 Hencke (2004), in: Peters (Hrsg.), Handbuch der Krankenversicherung, SGB V, § 140a Rn. 3. 
nahmevereinbarung Voraussetzung ist. 680 Festzuhalten bleibt allerdings, daß bereits bisher mindestens im Wege der Vereinbarung eine legitime Regelung möglich ist. Darüber hinaus ist mit $\S 53$ Abs. 3 SGB V eine Verbindung zur satzungsrechtlichen Gestaltung mindestens im tarifären Bereich geschaffen worden. Dabei geht die Gesetzesbegründung von der „Bindung an bestimmte Leistungserbringer“ aus. 681

\subsubsection{Werbung durch Krankenhäuser}

Das Wahlverhalten der Versicherten wird ferner - zunehmend - durch Werbung seitens der Krankenhäuser beeinflusst. Mit Blick auf die steigende Zahl wettbewerbsrechtlicher Streitigkeiten, ${ }^{62}$ den Wettbewerbsdruck der Krankenhäuser und die im Rahmen des Spezialisierungswettbewerbs besonders virulente Informationsasymmetrie sprechen zahlreiche Indizien für die Verhaltenssteuerung der Behandlungsbedürftigen durch Krankenhauswerbung. Das Verhalten der Patienten lässt sich danach umso stärker beeinflussen, je weiter der Rahmen normativ zulässiger Werbung gezogen ist.

Ohne Frage setzt das Sozialrecht, wie etwa die Anforderungen an die Qualitätsberichte gem. § 137 Abs. 3 [S. 1] Nr. 4 SGB V, dem Werbeverhalten Grenzen, wobei gerade dieses Beispiel als nunmehr „legalisierte“ Werbemaße mit dem Recht zur Veröffentlichung im Internet verstanden werden könnte, die den Wettbewerb befördern soll, wenn dieses nicht gleichzeitig durch eine entsprechende Pflicht flankiert würde.

Der insofern maßgebliche Standard folgt daher im wesentlichen aus den Restriktionen des ärztlichen Berufsrechts, ${ }^{683}$ vgl. v.a. $§ 27684$ der Musterberufsordnung für die deutschen Ärztinnen und Ärzte (MBO), 685 die für die Krankenhäuser weithin mittelbare Wirkung entfalten. ${ }^{686}$ Soweit dies der Fall ist, erfolgt die Einordnung der Regelungen der Berufsordnungen der Landesärztekammern als sog. „Marktverhaltensregelungen“ i.S.d. § 4 Nr. 11 (i.V.m. §3) UWG, was jeweils durch Auslegung zu ermitteln ist. ${ }^{687}$ Entsprechende Verstöße können berufsgerichtlich sowie durch Klagen gestützt auf die

680 Vgl. Engelhard (2000), in Hauck/Noftz, SGB V, K § 140a Rn. 23.

681 BT-Drucks. 16/3100, S. 108.

682 Vgl. den Bericht „Unlautere Methoden im Gesundheitswesen - Wettbewerbszentrale berichtet über Tätigkeit gegen Ärzte und Krankenhäuser“" (ohne Autor), KHuR 2004, S. 37 ff.

683 Siehe Spickhoff, in: Deutsch/Spickhoff, Medizinrecht, 6. Aufl. (2008), Rn. 38, 41 ff.; Bomba, Verfassungsmäßigkeit berufs- und standesrechtlicher Werbebeschränkungen für Angehörige freier Berufe (2003).

684 Dazu und zum Wortlaut der Vorschrift oben I.4.4.6.

685 Von grundlegender Bedeutung sind ferner die $\S \S 31-34$ MBO.

686 Vgl. Lehment, Werberecht für Krankenhäuser und Institute, Grenzüberschreitungen nicht erlaubt, DÄ 97 (2000), S. A 2916,

687 Siehe Köhler, in: Baumbach/Hefermehl, Wettbewerbsrecht, 25. Aufl. (2007), § 4 UWG Rn. 11.74. Der Begriff der gesetzlichen Vorschrift ist weit zu verstehen und erfaßt (u.a.) die von deutschen Gesetzgebungsorganen erlassenen Normen (s. Köhler, a.a.O., § 4 UWG Rn. 11.24). 
Verletzung des Gesetzes gegen den unlauteren Wettbewerb (UWG) durch (bewehrte) Unterlassungsbegehren und Schadensersatzansprüche ,geahndet“ werden. 688

Wenngleich dies nicht einhellig beurteilt wird, ${ }^{689}$ steht $\S 69$ SGB V hier der Anwendung des Wettbewerbsrechts nicht entgegen. Dies folgt einer strengen Interpretation, die Norm bestimme die Exklusivität der Vorschriften des 4. Kap. des SGB V nur für das Verhältnis der Krankenkassen zu den Leistungserbringern, nicht aber das betroffene Verhältnis der Leistungserbringer untereinander und zu den Patienten. 690 Aber selbst dann, wenn man die Norm ,,auch auf die Beziehungen von Leistungserbringern untereinander“ erstreckt, „,soweit es um Handlungen in Erfüllung des öffentlich-rechtlichen Versorgungsauftrags der Krankenkassen geht“, dürfte der „Erfüllungsbezug“ in dieser Konstellation nicht oder nur in seltenen Fällen direkt beworbener Vertragskonzepte ausreichen; einen solchen Fall sah der $B G H^{691}$ im Vollzug einer Handlung, über die sich Krankenkasse und Apotheke zur Durchführung der vereinbarten Versorgung verständigt hatte und die demgemäß konkurrierende Apotheken betraf.

$\mathrm{Da} ß$ bei alledem die Grenzen des Klinikwerberechts wesentlich weiter gezogen sind als bei niedergelassenen Ärzten, hat mehrere Gründe: Zum einen stellen sich für medizinische Einrichtungen schlicht besondere Zurechnungsfragen. So vermag das ärztliche Berufsrecht etwa im Hinblick auf MVZen prinzipiell nur für den einzelnen Arzt bzw. insoweit Maßstabswirkung zu entfalten, als MVZ von Vertragsärzten selbst geführt werden. ${ }^{692}$ Schwierig ist die Beurteilung des Verhaltens von Krankenhausärzten. Hier stellen sich noch nicht vollständig geklärte Fragen der Erstreckungswirkung berufsrechtlicher Werbeverbote mittels der Zuweisung der Störereigenschaft an juristische Personen, deren Führung die Ärzte zu berufswidrigem Handeln oder einer Duldung prinzipiell berufswidriger Handlungen verleiten. 693

Von größter Wichtigkeit aber ist die Differenzierung zwischen freiberuflicher Tätigkeit der niedergelassenen Ärzte und gewerblicher Tätigkeit der Kliniken. Die MBO legt zugrunde: „Der ärztliche Beruf ist kein Gewerbe. Er ist seiner Natur nach ein freier Beruf". ${ }^{694}$ Demgegenüber ist bereits im Rahmen der verfassungsrechtlichen Grundlagen darauf hingewiesen worden, daß das Betreiben eines Krankenhauses ein Gewerbe darstellt. 695 Auch im Zusammenhang mit Werbeverboten hat das $B V e r f G$ bereits vor den oben bezeichneten Entscheidungen hervorgehoben, im Gegensatz zu den niedergelasse-

688 Siehe auch Spickhoff, in: Deutsch/Spickhoff, Medizinrecht, 6. Aufl. (2008), Rn. 44.

689 Vgl. das Urteil des LG Osnabrück, Urt. v. 18.9.2006, AZ.: 18 O 487/06, abrufbar unter www.landgericht-osnabrueck.niedersachen.de.

690 Vgl. zur Interpretation des Bundeskartellamts im Fusionswettbewerb bereits oben I.4.4.1. sowie II.4.5.

691 BGH Urt. v. 23.2.2006, GRUR 2006, S. 517 ff. = NJW RR 2006, S. 1046 ff.

692 Ratzel, in: Dahm/Möller/Ratzel, Rechtshandbuch Medizinische Versorgungszentren (2005), Kap. VII. Rn. 2 ff.

693 Ratzel, a.a.O., Rn. 3 ff.; Spickhoff, in: Deutsch/Spickhoff, Medizinrecht, 6. Aufl. (2008), Rn. 44.

$694 \S 1$ Abs. 1 S. 2 und 3 MBO.

695 S.o. I. 4.3.1. 
nen Ärzten, für die die MBO gelte, seien Kliniken ,gewerblich tätig“ und erzielten „gewerbliche Umsätze“. 696 Das führe dazu, daß $§ 27$ der MBO im Lichte der Berufsfreiheit interpretiert werden müsse.

„Für Kliniken gelten damit nicht dieselben Werbebeschränkungen.“697 Auch der BGH urteilt: „Zwischen ambulanter und stationärer Behandlung bestehen erhebliche betriebswirtschaftliche Unterschiede, die es rechtfertigen, Kliniken und Sanatorien hinsichtlich der Werbung anders zu behandeln als niedergelassene Ärzte.“ 698

Damit führen die Bundesgerichte die Rechtsprechung zur „Sanatoriumswerbung“, der zufolge Kliniken bei der anpreisenden Werbung im Hinblick auf die Akquisition von Patienten gegenüber niedergelassenen Ärzten privilegiert werden, ${ }^{699}$ fort. Wo im einzelnen - auch innerhalb der umstrittenen Fallgruppen - die Grenzen zu ziehen sind, soll an dieser Stelle dahinstehen und der Hinweis genügen, daß Lockerungen auf dem Vormarsch sind, etwa im Bereich der Information über Zusatzleistungen oder des Herausstellens von Erfolgsquoten. 700

Konkrete Werbeverbote schließlich aktualisieren sich insbesondere im Gesetz über die Werbung auf dem Gebiete des Heilwesens (HWG). ${ }^{701} \mathrm{Zu}$ erwähnen sind v.a. das Verbot von Werbegaben $\left(\S 7\right.$ HWG) und Werbeverbote außerhalb der Fachkreise ${ }^{702}(\S$ 11 HWG). Untersagt sind insbesondere die Wiedergabe der Krankengeschichte ( $\$ 11$ Abs. 1 Nr. 3 HWG), die bildliche Darstellung von Personen in Berufskleidung (Nr. 4) oder ein bildlicher Vorher-Nachher-Vergleich (Nr. 5). ${ }^{703}$

\subsection{Besonderheiten im Recht der PKV}

Im Privatversicherungsrecht ist - „bei medizinisch notwendiger stationärer Heilbehandlung" - die „freie Wahl unter den öffentlichen und privaten Krankenhäusern“" allgemein normiert. Dies folgt aus $\S 4$ Abs. 4 der Musterbedingungen 2009 (MB/KK) des

696 BVerfG, Beschl. v. 4.7.2000, NJW 2000, S. 2734 (2735).

697 A.a.O.

698 BGH, Beschl. v. 11.71.2002, NJW-RR 2002, S. 1685 (1686).

699 Dazu Lehment, Werberecht für Krankenhäuser und Institute, Grenzüberschreitungen nicht erlaubt, DÄ 97 (2000), S. A 2916.

700 S. Balzer, Aktuelle Rechtsprobleme aus dem Arzt- und Klinikwerberecht, KHuR 2003, S. 87 ff.

701 S. Kieser, Werbung für Krankenhäuser, in: Lenz/Dettling/Kieser, Krankenhausrecht (2007), S. 246 ff.

702 „Fachkreise im Sinne dieses Gesetzes sind“ gem. § 2 HWG „Angehörige der Heilberufe oder des Heilgewerbes, Einrichtungen, die der Gesundheit von Mensch und Tier dienen, oder sonstige Personen, soweit sie mit Arzneimitteln, Medizinprodukten, Verfahren, Behandlungen, Gegenständen oder anderen Mitteln erlaubterweise Handel treiben oder sie in Ausübung ihres Berufes anwenden.“

703 S. auch Müller, Krankenhauswerbung und rechtliche Grenzen, Pflege- und Krankenhausecht 2006, S. $88 \mathrm{ff}$. 
Verbandes der PKV. ${ }^{704}$ Bereits vor Einführung der Musterbedingungen existierten ähnliche Regelungen einzelner Versicherungsunternehmen. ${ }^{705}$ Voraussetzung nach $\S 4$ Abs. $4 \mathrm{MB} / \mathrm{KK}$ ist lediglich, daß die Krankenhäuser ,unter ständiger ärztlicher Leitung stehen, über ausreichende diagnostische und therapeutische Möglichkeiten verfügen und Krankengeschichten führen“. Die MB/KK 76 verlangten zusätzlich, daß die Krankenhäuser nach allgemein anerkannten Methoden arbeiten. Führen die Krankenhäuser, welche die vorgenannten Voraussetzungen erfüllen, auch Kuren bzw. Sanatoriumsbehandlung durch und nehmen sie auch Rekonvaleszenten auf, kommt es für die Gewährung der tariflichen Leistungen auf eine entsprechende schriftliche Zusage durch den Versicherer vor Beginn der Verhandlung an, $\S 4$ Abs. $5 \mathrm{MB} / \mathrm{KK}$.

Unter Steuerungs- und Wettbewerbsgesichtspunkten erlauben die Regelungen, daß sich der Versicherungsnehmer bzw. Patient ,in die Klinik mit den modernsten und auch wirksamsten Behandlungsmethoden“ begibt. ${ }^{706}$ Diese Wahlalternative weist zugleich in Richtung Qualitäts- und Spezialitätswettbewerb, der - dem Grundgedanken zufolge zu effizienter Behandlung und auch zur Reduktion möglicher Folgekosten führt. Mutmaßlich auch deshalb wurden „Befürchtungen, das Risiko könne untragbar werden“, 707 von dem Gedanken einer Ausrichtung an den Möglichkeiten der modernen Medizin verdrängt, und die zusätzlichen Kosten nach bisherigen Erfahrungen und im Zeichen der Versicherungsmathematik als tragbar eingestuft. 708 Überdies ist „ein mittelbarer Einfluß auf die Auswahlentscheidung durch eine Einschränkung der Kostenerstattung bei Inanspruchnahme ... nicht kostengünstiger Krankenhäuser ... nicht bekannt“ ${ }^{\text {“ }} 709$

Im übrigen werden entsprechende „Risiken“ weiter dadurch zurückgehen, daß die krankenhausindividuellen Preisunterschiede - im Bereich der pflegesatzrechtlichen Vergütung für allgemeine Krankenhausleistungen im DRG-System - mit Blick auf ein landeseinheitliches Niveau in weitem Umfang nivelliert werden. Soweit die allgemeinen Krankenhausleistungen einheitlich und damit auch für die Behandlung privat Versicherter mit pauschalierten Festpreisen vergütet werden, vollzieht sich die freie Wahl des Krankenhauses vor allem am Maßstab der Qualität der Behandlung. Das im Rahmen der gesetzlichen Krankenversicherung kurz skizzierte Qualitätssicherungsregime gilt dabei für nach $\S 108$ SGB V zugelassene Krankenhäuser einheitlich für alle Patienten ${ }^{710}$.

704 Allgemeine Versicherungsbedingungen für die Krankheitskosten- und Krankenhaustagegeldversicherung, abgedruckt bei Rüffer/Halbach/Schimikowski, VVG, 2009, S. 1602 ff., ferner unter www.pkv.de.

705 Schoenfeldt/Kalis (ausgehend noch von den MB/KK 1994), in. Bach/Moser (Hrsg.), Private Krankenversicherung (2002), S. 251. Zur historischen Entwicklung der Musterbedingungen Moser, a.a.O., S. 2.

706 Schoenfeldt/Kalis, a.a.O., S. 252.

707 Schoenfeldt/Kalis, a.a.O., S. 251.

708 Schoenfeldt/Kalis, a.a.O., S. $251 \mathrm{f}$.

709 Bretthauer, Zusammenschlüsse von Krankenhäusern in der Fusionskontrolle, NJW 2006, S. 2884 (2885).

710 Letzteres ergibt nach dem KHRG wieder aus $\S 137$ Abs. 1 S. 1 SGB V und damit wieder entsprechend der Formulierung vor Änderung durch das GKV-WSG. 
Dieser Qualitätswettbewerb dürfte auch im Bereich der Wahlleistungen dominieren, sicherlich auch deshalb, weil jedenfalls im Bereich der ärztlichen Wahlleistungen nach Pflegesatzrecht grundsätzlich die GOÄ einheitlich zur Anwendung kommt. ${ }^{711}$ Mehr Spielraum dagegen besteht im Bereich der nichtärztlichen Wahlleistungen, wenngleich auch hier jedenfalls feste Fixpunkte der Preisgestaltung entwickelt wurden. ${ }^{712}$

\section{Die Wahl der Versicherung durch den Verbraucher}

\subsection{Gesetzliche Krankenversicherung}

\subsubsection{Wahl- und Wechselrechte}

Die Versicherungspflichtigen oder -berechtigen (freiwillig Versicherten) sind „Mitglied der von ihnen gewählten Krankenkasse“, § 173 Abs. 1 SGB V. Sie können grundsätzlich zwischen den Kassen aller Kassenarten wählen (Abs. 2), wobei sie teilweise örtlichen Beschränkungen unterliegen und bei den Betriebs- und Innungskrankenkassen dann, wenn sie betriebs- bzw. innungsfremd sind, für die Wahl dieser Kassen auf deren Öffnung (qua Satzung) angewiesen sind. Das Wahlrecht kann nach Vollendung des 15. Lebensjahres ausgeübt werden, § 175 Abs. 1 S. 3 SGB V.

Soweit die Ausübung des Wahlrechts ab dem Jahre 2002 erfolgt(e), sind Versicherungspflichtige und -berechtigte an die Wahl der Krankenkasse mindestens 18 Monate gebunden, § 175 Abs. 4 S. 1 SGB V. Allerdings kann die Mitgliedschaft abweichend davon früher gekündigt werden, wenn die Krankenkasse ab dem 1. Januar $2009^{713}$ einen Zusatzbeitrag erhebt, ihren Zusatzbeitrag erhöht oder ihre Prämienzahlung verringert (Abs. 4 S. 5); die Mitgliedschaft kann dann bis zur erstmaligen Fälligkeit der Beitragserhebung, der Beitragserhöhung oder der Prämienverringerung gekündigt werden. Auf dieses Kündigungsrecht sind die Mitglieder spätestens einen Monat vor Fälligkeit hinzuweisen (Abs. 4 S. 6), und kommt die Krankenkasse diese Pflicht nicht nach, verschieben sich für das betroffene Mitglied sowohl die Erhebung oder Erhöhung des Zusatzbeitrages als auch die Frist für die Ausübung des Sonderkündigungsrechts entsprechend, Abs. 4 S. 7. Bei unterbliebenem Hinweis auf eine Prämienverringerung hingegen verschiebt sich nach dem eindeutigen Wortlaut allein die Frist für die Ausübung des Kündigungsrechts, die Prämienverringerung wird aber nicht ausgesetzt. Ob das im Kontext der wettbewerbsorientierten Neuordnung der Finanzierungsströme konsequent er-

711 Zu den Einzelheiten Büsken, in: Bach/Moser (Hrsg.), Private Krankenversicherung (2002), MB/KK, nach $\S 1$, Rn. 5 ff.; Quaas, in: Wenzel (Hrsg.), Handbuch des Fachanwalts Medizinrecht (2007), Kap. 12, S. 1137 Rn. 84.

712 Quaas, a.a.O., Rn. 85 ff.

713 Nach alter Rechtslage galt als besonderer Kündigungsgrund, wenn die Kasse ihren kassenindividuell festgesetzten Beitrag erhöhte; Kündigungstermin war insoweit der Ablauf des auf das Inkrafttreten der Erhöhung folgenden Monats, § 175 Abs. 4 S. 5 SGB V a.F. 
scheint, mag dahinstehen, denn nach dem klaren Gesetzeswortlaut jedenfalls kommt eine Analogie nicht in Betracht.

Schließlich kann die Mitgliedschaft wiederum abweichend von den genannten Grundsätzen frühestens zum Ablauf der dreijährigen Mindestbindungsfrist gekündigt werden, wenn sich der Versicherte über Wahltarife entsprechend gebunden hat, §53 Abs. 8 S. 2 SGB V i.V.m. § 175 Abs. 4 SGB V. Die dreijährige Mindestbindungsfrist gilt allerdings nicht für Wahltarife im Zusammenhang mit besonderen Versorgungsformen („Managed Care“), § 53 Abs. 8 S. 1 i.V.m. Abs. 3 SGB V.

\subsubsection{Wahl der Krankenkasse aufgrund des Angebots krankenhausspezifischer Ver-}

$$
\text { sorgungskonzepte (IV, DMP) }
$$

Daß der Versicherte eine Krankenkasse mit Blick auf bestehende Vertragsbeziehungen zu einzelnen Krankenhäusern auswählt, dürfte (noch) die Ausnahme bilden. Möglich jedenfalls erscheint, daß sich der Versicherte gerade deshalb einer Krankenkasse anschließt, weil diese ihren Mitgliedern besondere Versorgungsformen anbietet, innerhalb derer die Zusammenarbeit mit einem bestimmten Krankenhaus bzw. bestimmten Häusern stattfindet. Damit erfolgt im Ergebnis eine Koppelung der Entscheidung für den Versicherer, das Versorgungskonzept und das teilnehmende Krankenhaus, vgl. $\S 140 \mathrm{a}$ Abs. 1, 4, § 140b Abs. 1 Nr. 2 SGB V. Als Anreiz mag dabei insbesondere das Versorgungsangebot unter spezifischer Einbindung des Krankenhauses dienen. Für das Gesamtkonzept spielt sicherlich auch die tarifäre Gestaltung eine Rolle, vgl. § 53 Abs. 3 SGB V. Danach hat die Krankenkasse zu regeln, daß für Versicherte, die an besonderen Versorgungsformen teilnehmen, besondere Tarife angeboten werden müssen. Darüber hinaus kann die Tarifierung für die Inanspruchnahme von Krankenhausleistungen auch eigenständige Bedeutung ohne spezifische Einbindung in ein Versorgungskonzept entfalten, etwa im Rahmen von Selbstbehalten. ${ }^{714}$

Entscheidender Auslöser für die Wahl der Versicherung könnte demnach durchaus das Versorgungskonzept der Krankenkasse im Hinblick auf die spezifische „Integration" des stationären Sektors sein. Hier kommen die IV und das Disease Management im Sinne der dafür besonders zugelassenen Programme in Betracht, wobei zu betonen ist, daß auch - und gerade - DMP-Strukturen durch Verträge zur IV umgesetzt werden. ${ }^{715}$

Was die $I V$ anbelangt, so erscheint hier weniger wahrscheinlich, daß ein Versorgungskonzept der bevölkerungsbezogenen und flächendeckende Vollversorgung, wie etwa das Modell „gesundes Kinzigtal“, 716 wegen des krankenhausspezifischen Bezugs den Willensentschluß des Versicherten hinsichtlich der Wahl der Krankenkasse maßgeblich trägt. Vielmehr dürften in dieser Hinsicht Projekte relevant werden, die als indi-

\section{Dazu sogleich unter 2.1.3.}

715 Sichert/Walser, Management of Chronic Diseases in the Netherlands and in Germany, European Journal of Social Security, Vol. 9 (2007), S. 243 (261 ff.).

716 Siehe bereits Fn. 182. 
kationsbezogenes (case-) Management den konkreten Lebens- und Krankheitsbedürfnissen des potentiellen Mitglieds Rechnung tragen. An dieser Stelle ließe sich eine Vielzahl von Beispielen anführen. Aus diesem Kreis früher Versorgungsangebote herausgegriffen sei etwa das Programm der BARMER Ersatzkasse, wie es gegenüber den Versicherten bzw. potentiell zu Versichernden angepriesen wurde: 717

„Das Programm... unterstützt Menschen mit bestimmten Krankheitsbildern, wie zum Beispiel Hüft- und Kniegelenkverschließ. Von der Diagnosestellung über die Operation und dem damit verbundenen Klinikaufenthalt bis hin zur Rehabilitation und Nachsorge: alle Beteiligten arbeiten eng zusammen. Das bietet viele Vorteile“".

Die endoprothetische Versorgung bildet in der Tat einen wichtigen Bereich der Beteiligung der Krankenhäuser an der IV, wobei in jüngerer Zeit die Problematik der Vereinbarung spezifischer Gewährleistungsklauseln zur Diskussion stand, die seitens der Krankenkassen vielfach gefordert wurden. ${ }^{718}$ Nach den Umfrageergebnissen des $D K I^{719}$ gaben $50 \%$ der befragten Häuser eine Beteiligung an der IV in diesem Bereich an. Andere Gebiete spielen eine deutlich geringere Rolle, und gerade dort erscheint z.T. weniger wahrscheinlich, daß die Wahl der Krankenkasse mit Blick auf die insofern vorhandenen bereichsspezifischen Konzepte beeinflusst würde: onkologische Versorgung $(13,9 \%)$, invasiv-kardiologische Versorgung (11,4\%), kardiochirurgische Versorgung (9,3\%), Sonstiges (46,9\%). Dazu passend werden als „Leuchtturmprojekte Integrierter Versorgung unter Beteiligung einzelner Krankenhäuser“ das „Das mammaNetz Augsburg“ (optimierte Brustkrebsversorgung), das Projekt für eine „Integrierte Versorgung Endoprothetik Münster“ und „Das Norddeutsche Herznetz“ (Integrierte Versorgung Kardiologie) beschrieben. ${ }^{720}$ Schließlich wird die Wahl der Krankenkassen wegen eines IV-Projekts mit Krankenhausbeteiligung durch einen aufgeklärten Patienten auch davon abhängen, ob die avisierte Kasse Vertrag und Konzept exklusiv anbietet (wie bei der Endoprothetik Münster die BARMER, vgl. oben) oder wie beim mammaNetz Augsburg 23 Kassen bzw. im Norddeutschen Herznetz BARMER, IKK, HEK und TK. 721

Die indikationsspezifische Betrachtungsweise gilt auch für das Angebot sog. strukturierter Behandlungsprogramme oder auch Disease Managementprogramme (DMP), die für chronische Krankheiten entwickelt worden sind. Bei der Einführung der Regelungen zur Steuerung mittels DMP ( $\S 137$ f, g, § 28b RSAV) durch das Gesetz zur Reform des RSA ging es darum, wettbewerbsspezifische Ineffizienten des Ausgleichssystems durch die Konzentration des Mitgliederwettbewerbs auf gesunde Versicherte („Herausselektion schlechter und Wettbewerb um gute Risiken") zu beseitigen und die Versorgung der chronisch kranken Versicherten durch Privilegierungen im Ausgleichssystem zu för-

717 Vgl. unter www.barmer.de (Leistungen und Beiträge, Integrierte Versorgung), Juni 2007.

718 Vgl. $D K G$, Empfehlung vom 31.8.2005, www.dkgev.de/dkgev.php/print/1/cat/112/aid/1396.

719 Krankenhaus Barometer, Umfrage 2006, September 2006, S. 54.

720 Vgl. die Darstellungen in Weatherly u.a. (Hrsg.), Leuchtturmprojekte Integrierter Versorgung und Medizinischer Versorgungszentren (2007), S. 45 ff.; 73 ff., 83 ff.

721 A.a.O., S. 76 bzw. 46 f. bzw. 84 f. 
dern. ${ }^{722}$ Zugelassen sind Programme für Brustkrebs (seit Februar 2003), Diabetes Mellitus Typ 2 (April 2004), Koronare Herzerkrankung (September 2004), Diabetes Mellitus Typ 1 (Mai 2005) und Asthma und COPD (Januar 2006). ${ }^{723}$ Im M-RSA ist diese spezifische Verknüpfung ungeachtet der Zuweisungen zur Deckung der Kosten nach $\S 270$ Abs. 1 lit. b SGB V über die Morbiditätsorientierung hinaus nicht mehr gegeben.

Die Beteiligung der Krankenhäuser an DMP fällt unterschiedlich aus. Viele DMPVerträge, etwa im Bereich des Diabetes, werden im Grundvertrag ohne die Beteiligung der Krankenhäuser geschlossen, diese im Gesamtprogramm dann aber beteiligt, u.a. aufgrund direkter Verträge bzw. Beziehungen der Kassen zu den Krankenhäusern. Insgesamt bestehen für alle zugelassenen DMP vertragliche Beziehungen zu Krankenhäusern, welche durch eine Vielzahl von Selektivvertragstypen umgesetzt werden können. ${ }^{724}$ Dabei spielt in besonderer Weise die ambulante Behandlung im Krankenhaus eine Rolle, für die $\S 116 \mathrm{~b}$ Abs. 1 SGB V besondere Verträge mit den an DMP teilnehmenden Krankenhäusern vorsieht.

Die maßgeblichen Erhebungen zur Beteiligung der Krankenhäuser an DMP stammen (noch) aus dem Jahre 2005. ${ }^{725}$ In Ansehung des Zeitrahmens der (jüngeren) Zulassung der einzelnen Programme lassen sich stete Entwicklungstrends zur Beteiligung der Häuser und zur relativen Bedeutung der einzelnen DMP noch kaum ablesen. Dominierte im Jahre 2004 mit 13,1\% noch die Beteiligung am DMP Brustkrebs, gefolgt von Diabetes mellitus Typ 2 (9,9\%) und Koronarer Herzerkrankung (0,5\%), nahmen im Jahr darauf DMP-Verträge zu Diabetes mellitus Typ 2 den ersten (28,1\%), zu Brustkrebs den zweiten $(25,5 \%)$ und für die Herzerkrankungen den dritten Rang ein $(16,1 \%)$, gefolgt von DMP-Verträgen über chronische Atemwegserkrankungen (2,5\%). Bereits zum 1.1.2004 entfaltete der flächendeckende Vertrag zu Brustkrebs in Baden-Württemberg Wirkung. ${ }^{726}$

Während die Krankenkassen nach Einführung der DMP bekanntermaßen stark damit geworben haben, diese Programme für ihre Versicherten, vor allem für Diabetiker, vorzuhalten, ist wenig bekannt, inwiefern die Einbindung des stationären Sektors Einfluß auf eine Kassenwahlentscheidung gehabt haben könnte. Jedenfalls aber dürfte der Aspekt umfassenden Versorgungsmanagements - auch für den Fall des ungünstigen Krankheitsverlaufs - nicht als unmaßgeblich von der Hand zu weisen sein.

722 Ausf. Sichert/Walser, Management of Chronic Diseases in the Netherlands and in Germany, European Journal of Social Security, Vol. 9 (2007), S. 243 (256 ff.).

723 Dazu m.w.N. Sichert/Walser, S. 259 ff.

724 Ausf. N. a.a.O., S. $261 \mathrm{ff}$.

725 DKI (Hrsg.), Krankenhaus Barometer, Umfrage 2005, November 2005, S. 37 ff.

726 Geschlossen zwischen Landesverbänden der Kassenkassen bzw. der AOK Baden-Württemberg und allen Kassenärztlichen Vereinigungen sowie der Baden-Württembergischen Krankenhausgesellschaft (auf der Grundlage des $\S 115$ SGB V); siehe unter www.senologie.org/download /pdf/dmpbrustkrebs200204.pdf. 


\subsubsection{Wahl der Krankenkasse aufgrund tarifärer Privilegierung der}

Nichtinanspruchnahme stationärer Leistungen?

Ungeachtet des Angebots krankenhausspezifischer Versorgungskonzepte und der Vermittlung privater Zusatzversicherungen zur stationären Versorgung 727 kann die Tarifgestaltung der gesetzlichen Krankenkassen auch insoweit (indirekt) Anreize setzten, als die Nichtinanspruchnahme stationärer Leistungen privilegiert wird.

Die in diesem Zusammenhang maßgeblichen Tarifgestaltungen wie Boni für gesundheitsbewußtes Verhalten ( 65a SGB V) und Wahltarife mit Selbstbehalt ( 53 SGB V) zielen darauf $a b$, daß das versicherte Mitglied nach eigener Einschätzung seines Krankheitsrisikos in Verbindung mit der wirtschaftlichen Beurteilung der Kosten im Krankheitsfall gerade nicht davon ausgeht, der stationären Behandlung zu bedürfen. Allerdings sind die genannten Tarifoptionen eher selten speziell auf den stationären Sektor zugeschnitten, sondern betreffen die Behandlungsbedürftigkeit bzw. Inanspruchnahme von Leistungen im ambulanten wie im stationären Bereich. ${ }^{728}$

So erhält das versicherte Mitglied bspw. den seitens der AOK Bayern offerierten Grundbonus ( $§ 65 \mathrm{a}$ SGB V) in voller Höhe von $€ 100$ p.a., ${ }^{729}$ wenn weder er noch die Mitversicherten ambulante Behandlung (die mit einer Arzneimittelversorgung einher geht) oder Krankenhausbehandlung in Anspruch nehmen. ${ }^{730}$ Gleichzeitig wird im Falle der Behandlungsbedürftigkeit eine - über die gesetzliche Zuzahlung hinausgehende Eigenbeteiligung je Krankenhausbehandlung von $€ 50$ fällig. Im Gesamtpaket dieses Beispiels ist auch ein Gesundheitsbonus von $€ 70$ p.a. enthalten, der u.a. die Teilnahme des Versicherten und der Mitversicherten an Vorsorge- und Früherkennungsuntersuchungen voraussetzt. Dieser Tarif lässt sich im Beispielsfall nicht mit dem vor der Krankenkasse angebotenen Wahltarif Selbstbehalt (§ 19m der Satzung) kombinieren. Je nach beitragspflichtigem Einkommen kann der Versicherte einen Grundbonus von bis zu $€ 500 \mathrm{im}$ Jahr erhalten, müßte in dieser höchsten Tarifklasse (ab € 42.001) allerdings auch den höchsten Selbstbehalt von $€ 125$ bei ambulanter Behandlung i.V.m. einer Arznei- oder Heilmittelverordnung und $€ 250$ je Krankenhausaufenthalt ${ }^{731}$ bezahlen. Das wirtschaftliche Risiko wäre in dieser Tarifklasse durch einen Höchstbetrag der zu tragenden Selbstbehalten von $€ 575$ begrenzt, und der Betreffende könnte auch eine niedrigere Tarifklasse mit geringeren Selbstbehalten und Höchstbeträgen - aber auch geringeren Grundboni - wählen.

727 Dazu nachfolgend 2.1.4.

728 Vgl. etwa $\S 19 \mathrm{j}$ und $\S 19 \mathrm{~m}$ der Satzung der AOK Bayern, Stand: 1.1.2009.

729 A.a.O., § 19j Abs. 3. Der Bonus ist gestaffelt und erhöht sich bis auf $€ 130$ p.a. in den Vorjahren bei fortwährender Nichtinanspruchnahme. Liegt das Einkommen des Mitglieds über einem Zwölftel der Jahresarbeitsentgeltgrenze, beträgt der entsprechende Bonus $€ 200$ bis $€ 230$.

730 Der Höchstbetrag aller Beteiligungen beträgt $€ 250$. Liegt das Einkommen des Mitglieds über einem Zwölftel der Jahresarbeitsentgeltgrenze, liegt der Höchstbetrag bei $€ 350$, § 19j Abs. 5.

$731 \S 19 \mathrm{~m}$ Abs. 4 der Satzung spricht von „Krankenhausbehandlung“. 
Alles in allem ist grundsätzlich davon auszugehen - und dies entspricht der gesetzgeberischen Intention -, daß das Angebot an Wahltarifen auch die Wahl der Krankenkasse bestimmt. Inwiefern dies aber mit Blick auf den stationären Sektor der Fall sein könnte (vgl. auch II.2.1.6.), ist bis dato weder umfassend untersucht noch evident.

\subsubsection{Die Vermittlung privater Zusatzversicherungen für die}

\section{Krankenhausbehandlung seitens der Krankenkassen}

Mit Einführung des GMG haben die gesetzlichen Krankenkassen die Möglichkeit erhalten, den Abschluß privater Zusatzversicherungen zwischen den Versicherten und privaten Krankenversicherungsunternehmen zu vermitteln, § 194 Abs. 1a SGB V. Dies setzt einerseits den Bezug zur gesetzlichen Versicherung voraus, andererseits sind Vertragspartner allein der Versicherte und das private Unternehmen, wobei die Grundsätze des privaten Versicherungsrechts maßgeblich sind. ${ }^{732}$ Diese Zusatzversicherungen entfalten gerade im Hinblick auf die Krankenhausversorgung besondere Bedeutung: Gem. $\S 194$ Abs. 1a S. 2 SGB V können „Gegenstand dieser Verträge... insbesondere die Wahlarztbehandlung im Krankenhaus, der Ein- oder Zweibettzuschlag im Krankenhaus sowie eine Auslandsreisekrankenversicherung sein“. Ein solcher „stationärer Zusatzschutz"733 wird von den gesetzlichen Krankenkassen nahezu durchgängig (als Vermittlungspaket) angeboten. Dies beeinflusst auch den angebotsorientierten Wettbewerb der gesetzlichen Krankenkassen untereinander. Speziell unter dem Gesichtspunkt des Mitgliederwettbewerbs wird zugleich für die Zusatzangebote, die in Abhängigkeit der Kooperation mit den privaten Partnern häufig auch differenziert ausfallen, verstärkt geworben; 734 die Mitgliedschaft in einer bestimmten Krankenkasse präsentiert sich so als privilegierter Zusatzversicherungs-Optionsstatus, insbesondere im Zusammenhang mit einem speziellen Tarif. Kooperationen der insoweit marktmächtigen gesetzlichen Krankenkassen mit den PKV-Unternehmen eröffnen die Möglichkeit, für die Versicherten attraktive Gruppentarife auszuhandeln. In welchem Umfang die einzelnen für den ,stationären Zusatzschutz" maßgeblichen Wettbewerbsparameter die Entscheidung für die Mitgliedschaft in einer bestimmten gesetzlichen Krankenkasse beeinflussen, lässt sich kaum ergründen. Bereits die Einführung des § 194 Abs. 1a SGB V aber zeigte, daß ein maßgeblicher Wunsch der Versicherten für die Ergänzung bestand. Danach ist jedenfalls davon auszugehen, daß Existenz und Zuschnitt des Vermittlungspaketes „stationärer Zusatzschutz" die wettbewerbsorientierte Entscheidung für die Wahl einer gesetzlichen Krankenkasse durchaus mitbestimmen können.

732 Vgl. oben I. 1.1. sowie II. 2.1.

733 Terminologie der Barmer, vgl. unter www.barmer.de (Leistungen und Beiträge, Zusatzversicherung).

734 Vgl. wiederum Tarife der AOK Bayern „AOK-KlinikPRIVAT/1“ und „AOK-KlinikPRIVAT/2“, www.aok.de/bay/rd/129794.htm. 


\subsubsection{Mitgliederwerbung}

Krankenkassen betreiben in immer größerem Umfang Werbung. Diese ist in nur geringem Umfang Werbung etwa für besondere Versorgungsangebote der bereits Versicherten, v.a. aber Mitgliederwerbung. Aus Sicht der versicherten, kassenindividuell „zu haltenden“ bzw. der potentiellen Neumitglieder führt Werbung damit - im günstigen Fall - zu einer Korrektur der Informationsasymmetrie. Da und soweit der Informationsgehalt der jeweiligen Werbeaktivität indes zuvorderst auf die nach wirtschaftlichen Gesichtspunkten kalkulierte Wahl nur der werbenden Kasse ausgerichtet ist, kommt die Frage der Kontrolle der Werbeaktivitäten auf. Diese ist Ausfluss des Schutzes auch der Versicherten, betrifft allerdings zuvorderst das Wettbewerbsverhältnis der Krankenkassen zueinander und wird daher unter 5.1.3. behandelt.

\subsubsection{Wahltarif „Kostenerstattung für Chefarztbehandlung bzw. Ein-/} Zweibettzimmer"?

Eine mit Blick auf die Leistungsdiversifikation für Krankenhausbehandlung besonders wichtige und strittige Frage betrifft die Zulässigkeit von Wahltarifen bei Kostenerstattung ( 53 Abs. 4 SGB V) für „Chefarztbehandlung“ bzw. „Ein- oder Zweibettzimmer". 735 Sie beschränkt sich in ihrer grundlegenden Bedeutung nicht allein auf eine Wahloption innerhalb des Versicherungsverhältnisses, sondern entfaltet Bedeutung sowohl für den GKV-internen als auch den interaktiven Wettbewerb zwischen GKV und PKV. Wahltarife nach $\S 53$ SGB V, insbesondere solche der beschriebenen Art, werden oftmals als Ausdruck der Konvergenz der Systeme, kritisch aber v.a. als systemwidriges oder gar verfassungswidriges Vordringen der GKV in den Bereich der PKV gewertet. ${ }^{736}$ Sie sind ebenso Gegenstand gerichtlicher Auseinandersetzung zwischen GKV und $\mathrm{PKV}^{737}$ wie eine von $30 \mathrm{PKV}$-Unternehmen erhobene Verfassungsbeschwerde jüngst in diesem Punkte für unzulässig befunden wurde. 738

Will man die Zulässigkeit des Wahltarifs „Kostenerstattung für Chefarztbehandlung bzw. Ein-/Zweibettzimmer“ isoliert betrachten, hängt einiges von der (Vor-)Frage ab,

735 Vgl. etwa $\S 28$ Abs. 4 der Satzung der AOK Rheinland/Hamburg v. 1.1.2009 (www.aok.de): Tarif für die Kostenerstattung bei Wahlleistung „Ein- oder Zweitbettzimmer“. Die Monatsprämien für Zwei-/Einbettzimmer belaufen sich auf $€ 4,70 / 7,80$ (bis zur Vollendung des 20. Lebensjahres) bis zu $€ 52,00 / 85,20$ (nach Vollendung des 65. Lebensjahres), vgl. § 28 Abs. 6 i.V.m. Anhang 2 Ziff. 3 der Satzung.

736 Vgl. Isensee, Wahltarif "Krankenhauskomfort" - Chefarztbehandlung und Ein-/Zweibettzimmer als Wahlleistungen der Kassen - Sicht des Sozial- und des Verfassungsrechts, NZS 2007, S. 449 ff.

737 Vgl. LSG NRW, Beschl. V. 27.05.2008, L 11 B 6/08 KR ER.

738 Mangels Beschwer bzw. unmittelbarer Betroffenheit, BVerfG, Urt. v. 10.6.1009, 1 BvR 706/08, 1 BvR 814/08, 1 BvR 819/08, 1 BvR 832/08, 1 BvR 837/08, Rn. 133 f. (juris).S. ferner Isensee, Wahltarif "Krankenhauskomfort" - Chefarztbehandlung und Ein-/Zweibettzimmer als Wahlleistungen de Kassen - Sicht des Sozial- und des Verfassungsrechts, NZS 2007, S. 449 (452 ff.). 
ob es sich bei diesem „Modus der Behandlung und Unterbringung“ 739 tatsächlich um eine nach normativen Maßstäben system- und rechtswidrige Leistungsausweitung handelt. ${ }^{740} \mathrm{Im}$ übrigen lässt sich nicht argumentieren, weil die Tarife u.U. Auswirkungen auf die Beurteilung der Tätigkeit der Krankenkassen als Unternehmen hätten, sei eine solche Ausgestaltung nicht zulässig bzw. gewollt gewesen. ${ }^{741}$

Wichtig ist danach zunächst, daß Wahltarife allein für die Einzelleistungen Chefarztbehandlung oder Zweibettzimmer in der Tat eine unzulässige Leistungsausweitung darstellen; auch das Schreiben des BVA vom 13. März $2007^{742}$ ist allein dahingehend zu verstehen, daß die Einbeziehung der Chefarztbehandlung und der Unterbringung im Zweibettzimmer nur insoweit möglich sind, als insgesamt Kostenerstattung für Versorgung mit Krankenhausleistungen gewählt wird. ${ }^{743}$ Da die weitergehende Diskussion im wesentlichen mit Fragen der Zulässigkeit nach Art der Leistungserbringung, als Zusatzversicherung sowie des Eindringens in den Bereich der PKV beschrieben wird, soll sie im Rahmen des Konkurrenzverhältnisses GKV-PKV fortgeführt werden. ${ }^{744}$

\subsection{Besonderheiten im Recht der PKV}

Daß krankenhausspezifische Aspekte die Wahl des PKV-Unternehmens bestimmten, dürfte mindestens ebenso zurückhaltend zu beurteilen sein wie im Bereich der GKV. Zwar ist darauf hinzuweisen, daß „Managed Care“ keinesfalls eine Domäne allein der GKV ist. Das gilt speziell auch für die DMP. ${ }^{745}$ Diese allerdings schlagen der Natur der PKV-Verhältnisse entsprechend nicht auf das versicherungsrechtliche Grundverhältnis „durch“ und beeinflussen den Mitgliederwettbewerb in aller Regel nicht. ${ }^{746}$

Unbeschadet dessen kann der Versicherungsnehmer ein Krankenversicherungsverhältnis, das für die Dauer von mehr als einem Jahr eingegangen ist, zum Ende des ersten Jahres oder jedes darauf folgenden Jahres unter Einhaltung einer Frist von drei Monaten kündigen, soweit keine Mindestversicherungsdauer vereinbart worden ist, § 205 Abs. 1 S. 1 VVG (2009). ${ }^{747}$ Die Kündigung kann auf einzelne versicherte Personen oder Tarife beschränkt werden (S. 2). Diesen Vorschriften entsprechen $\S 13$ Abs. 1 und 2 MB/KK (2009). Für die normalerweise auf unbestimmte Zeit geschlossenen Verträge

739 Isensee, a.a.O., S. 450.

740 So v.a. Isensee, a.a.O.

741 Vgl. Schlegel (2007), jurisPK SGB V, § 53 Rn. 116.

742 Az. II 1 - 4927.6 - 3709/2006.

743 Beckschäfer, Die Ersatzkasse 2008, S. 438 (439).

744 S. unter II. 5.3.

745 Benner (2003), Disease-Management-Programme. Unterschiedliche Ansätze von privater und Gesetzlicher Krankenversicherung, Deutsches Ärzteblatt, Vol. 100, Heft 3, S. A82-A84.

746 Siehe näher unten 3.2.

747 Vormals $\S 178 \mathrm{~h}$ Abs. 1 S. 1 VVG. $\S \S 193$ ff. (vormals $\S \S 178 \mathrm{a}$ ff. VVG) gelten auch für komplementäre Versicherungen, vgl. Römer, in: ders./Langheid, VVG, 2. Aufl. (2003), Vorbemerkung zu $\S \S 178 \mathrm{a}$ ff. Rn. 1. 
gelten $\S 13$ Abs. 1 und 2 MB/KK (2009) ebenso. 748 Die Neuregelung des $\S 11$ VVG macht im Gegensatz zur früheren Rechtslage deutlich, daß $\S 13 \mathrm{BM} / \mathrm{KK}$ nicht im Zusammenhang mit dieser Norm (vormals $\S 8$ Abs. 2 VVG) ${ }^{749}$ steht, sondern mit $\S 205$ $\mathrm{VVG}^{750}$ (vormals $\S 178 \mathrm{~h}$ Abs. 1 VVG). ${ }^{751}$

Für Fragen der Krankenhauswerbung schließlich gelten dieselben wie im Bereich der GKV konstatierten Grundsätze.

\section{Wettbewerbliche Beziehungen zwischen Kostenträgern und Krankenhäusern}

\subsection{Gesetzliche Krankenversicherung}

\subsubsection{Das Grundverhältnis zwischen Krankenkassen und Krankenhäusern}

Das Verhältnis der gesetzlichen Krankenkassen zu den Krankenhäusern fußt auf einem integralen sozialrechtlichen Zulassungs- und Vertragssystem, das gesetzliche und kontraktuelle Komponenten miteinander verbindet. Im Bereich des GKV dürfen Krankenkassen Krankenhausleistungen nur durch zugelassene Krankenhäuser i.S.d. § 108 Nr. 1-3 SGBV erbringen lassen. Das zugelassene Krankenhaus ist im Rahmen seines Versorgungsauftrages zur Krankenhausbehandlung verpflichtet, § 109 Abs. 4 S. 2 SGB V. Die Zulassung wiederum erfolgt „mit einem Versorgungsvertrag“, § 109 Abs. 4 S. 1 SGB V.

Ein solcher Versorgungsvertrag kommt auf zweierlei Weise unterschiedlich zustande. ${ }^{752}$ Der Abschluß erfolgt je nach Art der Krankenhäuser quasi automatisch, d.h. im Wege einer gesetzlichen Fiktion, oder aber durch „echten“, konsensualen Vertragsschluß.

Demnach gilt bei den Hochschulkliniken die Anerkennung nach den landesrechtlichen Vorschriften ( $§ 108$ Nr. 1 SGB V) als Abschluß des Versorgungsvertrages, ebenso wie bei den Plankrankenhäusern ( $§ 108$ Nr. 2 SGB V) die Aufnahme in den Krankenhausbedarfsplan, § 109 Abs. 1 S. 2 SGB V. Mit den übrigen Krankenhäusern kann ein Versorgungsvertrag zwischen den Landesverbänden der Krankenkassen und den Verbänden der Ersatzkassen schriftlich geschlossen werden, § 109 Abs. 1 S. 1 SGB V. Umgekehrt werden diese Krankenhäuser mit dem Vertragsschluß zugelassen, § $108 \mathrm{Nr}$. 3 SGB V; ein solcher Vertrag darf indes nicht geschlossen werden, wenn das Krankenhaus nicht die Gewähr für eine leistungsfähige und wirtschaftliche Krankenhausbehand-

748 Siehe Moser, in: Bach/Moser, Private Krankenversicherung, 3. Aufl. (2002), § 13 MBKK Rn. 14 ff.

749 So Prölls, in: ders./Martin, Versicherungsvertragsgesetz, 27. Aufl. (2004), § 178h Rn. 1, dies führte früher zum selben Ergebnis.

750 S. Muschner, in: Rüffer/Halbach/Schimikowski, VVG (2009), § 11 Rn. 2; Rogler, a.a.O., § 1 $\mathrm{MB} / \mathrm{KK}, \mathrm{Rn} .1 \mathrm{f}$.

751 Siehe Römer, in: ders./Langheid, VVG, 2. Aufl. (2003), § 8 Rn. 28.

752 Zur Kündigung $§ 110$ SGB V. 
lung bietet oder für eine bedarfsgerechte Krankenhausbehandelung der Versicherten nicht erforderlich ist, $\S 109$ Abs. 3 S. 1 SGB V. Der Abschluß und die Ablehung des Versorgungsvertrages werden mit der Genehmigung durch die zuständigen Landesbehörden wirksam, § 109 Abs. 3 S. 2 SGB V.

Im Bereich der Regelversorgung wird damit deutlich, daß Planung und Versorgung (und somit Landes- und Bundesrecht) ebenso wie Vertrag und Gesetz eng miteinander verwoben sind. Dem entspricht der bereits im Bereich des Krankenhauswahlrechts der Versicherten konstatierte Befund einer planungsgeleiteten materiellen Begrenzung dieses Wahlrechts. Mit der Einschränkung gem. § 109 Abs. 3 S. 1 SGB V wird darüber hinaus auch der Spielraum zum Vertragsschluß für „Vertragskrankenhäuser im engeren Sinne“753 Erwägungen der Wirtschaftlichkeit und Bedarfsgerechtigkeit unterworfen, und zwar im Verhältnis zu den Hochschulkliniken und Plankrankenhäusern gleichsam auf einer nachgeordneten zweiten „Stufe“.

\subsubsection{Besonderheiten im ambulanten Bereich}

Der im Hinblick auf die Zulassung statusbegründende Vertrag 754 entfaltet Bedeutung für die Krankenhausbehandlung insgesamt, nicht nur für den stationären Bereich. Dies folgt - unbeschadet der lückenhaften Aufzählung - aus § 39 SGB V (i.V.m. § 109 Abs. 4 S. 2 SGB V) und einem weiten Begriff der Krankenhausbehandlung 755 ebenso wie aus der Inbezugnahme des ,zugelassenen“ Krankenhauses etwa in $\S 116 \mathrm{~b}$ Abs. 1 und 2 SGB V. Vor diesem Hintergrund sind mit Blick auf einzelvertragliche Optionen der zulassungsbegründende Grundvertrag und - soweit zulässig - der Selektivvertrag voneinander zu unterscheiden. Selektivverträge, also Verträge (jedenfalls auch) einzelner Krankenkassen mit (einzelnen) Krankenhäusern, ${ }^{756}$ mit Abschluß- und (mindestens partieller) Gestaltungsfreiheit sind allein im ambulanten Bereich oder aber - krankenhausspezifisch - als Integrationsverträge zur Überwindung der Grenzen zwischen ambulanter und stationärer Versorgung vorgesehen.

Das bedeutet jedoch nicht, daß die ambulante Versorgung grundsätzlich selektivvertraglich organisiert wäre. Im Gegenteil wird die Berechtigung zuvorderst allgemein reguliert (vgl. § 115b Abs. 2 SGB V), und der maßgebliche Katalog der ambulanten Operationen im Krankenhaus und eine entsprechende einheitliche Vergütung werden durch dreiseitigen Vertrag auf Bundesebene vereinbart, § 115b Abs. 1 SGB V. § 116 SGB V betrifft die besondere Ermächtigung der Krankenhausärzte, $\S 116 \mathrm{~b}$ diejenige der Krankenhäuser zur ambulanten Behandlung im Falle der Unterversorgung.

Darüber hinaus hat der Gesetzgeber die vormalige Vertragskompetenz der Krankenkassen, mit zugelassenen Krankenhäusern Verträge über die ambulante Erbringung

753 Vgl. Quaas, in: Wenzel (Hrsg.), Handbuch des Fachanwalts Medizinrecht (2007), Kap. 12 Rn. 153.

754 Rau (2007), in: O/R/S/W/Z, SGB V, § 108 Rn. 3.

755 Vgl. Quaas, in: Wenzel (Hrsg.), Handbuch des Fachanwalts Medizinrecht (2007), Kap. 12 Rn. 66.

$756 \mathrm{Zu}$ diesen zählen Verträge nach $§ 108$ Nr. 3 SGB V nicht. 
hochspezialisierter Leistungen sowie zur Behandlung seltener Erkrankungen und Erkrankungen mit besonderen Krankheitsverläufen zu schließen (§ 116b SGB V), abgeschafft. Das Pendel schlägt hier wieder in Richtung Regulierung aus, wenn nunmehr gilt: „Die Entscheidung [über den Antrag] fällt im Rahmen der Krankenhausplanung“. 757

\subsection{3. „Regulierungsverflechtung“"758}

$\mathrm{Da}$ es im Bereich der stationären Krankenhausversorgung entgegen anders lautenden Empfehlungen ${ }^{759}$ nicht zu einer Weiterentwicklung des Vertragswettbewerbs gekommen ist, hat offenkundig auch damit zu tun, daß strukturerhebliche Fragen wie etwa das Verhältnis zum Kollektivvertragssystem, Fragen der Bereinigung der Vergütung u.a. eher als Hindernisse angesehen, gleichzeitig indes regulatorisch in besonderer Weise aufgegriffen werden. So ist gerade im Zeichen der Verknüpfung der Berechtigung zur Erbringung hochspezialisierter Leistungen mit Planungsentscheidungen der Landesbehörden (§ 116b Abs. 2 S. 1 SGB V) ein zunehmendes Maß an „Regulierungsverflechtung" zu konstatieren, d.h. sowohl der sozialrechtlichen mit planerischen Erwägungen als auch zwischen der Bundes- und der Landesebene. Daß dies keine Momentaufnahme ist, zeigt auch der (nicht auf den ambulanten Bereich beschränkte) Fall, daß die zuständige Landesbehörde aus Gründen der Sicherstellung Ausnahmen von der Abrechnungsfähigkeit der einer Mindestmengenregelung unterliegenden Leistungen treffen und damit in die grundsätzlich sozialrechtlich determinierte Qualitätssicherung hineinwirken kann (vgl. § 137 S. 1 Nr. 2, S. 3 SGB V). Diese Regulierungsverflechtung wird zunehmend kritisiert. Unbeschadet der bedingt tragfähigen verfassungsrechtlichen Bedenken jedenfalls gerät - rechtspolitisch gesehen - die Legitimation des „Ordnungsgefüges“ Krankenhausplanung zunehmend unter Druck. ${ }^{760}$

\subsubsection{Vertragswettbewerb}

\subsubsection{Allgemeines}

Für echten Vertragswettbewerb, ja überhaupt Wahloptionen in der Handlungsform des Einzelvertrages bleibt demnach, praktisch betrachtet, relativ wenig Raum. Dies steht, jedenfalls für den Krankenhausbereich, in gewissem Gegensatz zur Grundintention des „Wettbewerbsstärkungsgesetzes“. Wo allerdings Vertragswettbewerb in gewis-

757 S. BT-Drucks. 16/3100, S. 139.

758 In Anlehnung an Scharpf, Theorie der Politikverflechtung, in: ders,/Reissert/Schnabel, Politikverflechtung (1976), S. $13 \mathrm{ff}$. (30 ff.).

759 Cassel u.a., Weiterentwicklung des Vertragswettbewerbs in der gesetzlichen Krankenversicherung (2006), S. 56 f.

760 Siehe Stollmann, Mindestmengenregelung nach § 137 SGB V - Ausnahmeentscheidung der Planungsbehörde, GesR 2007, S. 303 (304 ff.); Wenner, Einbeziehung von Krankenhäusern in die ambulante ärztliche Versorgung, GesR 2007, S. 337 (342 f.). 
sem Rahmen zulässig ist, treten Krankenhäuser als Vertragspartner mit Managementkapazitäten auf.

Vertragswettbewerb bedeutet mehr als „Wettbewerb mittels selektiver Verträge“; jedenfalls wird seine theoretische Dimension in dieser verkürzten Form kaum deutlich. Fokussiert auf das Recht der Leistungserbringer bedeutet direkter Vertragswettbewerb zunächst partnerspezifisch Wettbewerb um den Abschluß von Selektivverträgen zugunsten leistungsstarker Erbringer und Kostenträger. Inhaltsorientiert geht es um einen Wettbewerb der Vertragsmodelle, so zum Bespiel „Gesellschafts-“, „Kooperations-“ und „Einkaufsmodelle“ bzw. „Case-“, „Care“ oder globales Versorgungsmanagement. Gleichzeitig kommt es zur Konkurrenz der Vertragstypen. Diese Konkurrenz der Selektivvertragstypen - also der nach Abschlußart und Vertragspartnerschaft identischen, aber in puncto Inhalt, Gestaltungsfreiheit, Abrechnungsvorgaben etc. nach der sozialrechtlichen Vertragsgrundlage unterschiedlich ausgestalteten konsensualen Vertragsform - ist bisher eher wenig beleuchtet worden. ${ }^{761}$ Dasselbe gilt für eine Kombination der Vertragstypen, sowie - speziell vor dem Hintergrund der allgemeinen Regelungen der $\S \S 53$ ff. SGB X - für einen Vertragstypenzwang. Das Potential weitgehender Gestaltungsfreiheit kommt im ambulanten Bereich nunmehr in $\S 73 \mathrm{c}$ SGB V in Verträgen zur besonderen ambulanten Versorgung zum Ausdruck.

Der Gesetzgeber selbst ist auf die Weiterentwicklung bedacht und „sozialisiert“ die Ergebnisse von best practice-Modellen, indem er inhaltliche Vorgaben macht, z.B. mit Blick auf die Kombination von IV- und Rabattverträgen (§ 140a Abs. 1 S. 5 i.V.m. $\S$ 130a Abs. 8 SGB V), oder wenn $\S 73 b$ Abs. 1 SGB V klar bestimmt: „Die Krankenkassen haben ihren Versicherten eine... hausarztzentrierte Versorgung anzubieten“.

Darüber hinaus kennzeichnet den Vertragswettbewerb ein Wettbewerb der „Binnenintegration" der Leistungserbringer, um sich zu strukturell verhandlungsstarken Gemeinschaften zusammenzuschließen, die gemeinsam für Kostenträger attraktive Versorgungsangebote offerieren. Diese Binnen- oder „Vorab-Integration“ ließe sich auch als mittelbarer Vertragswettbewerb bezeichnen. Eine solche wettbewerbsorientierte, partnerschaftliche Kooperation unterscheidet sich von der ,korporatistischen Kooperation“, wie sie dem Kollektivvertragsbereich als Strukturelement innewohnt. ${ }^{762}$

Angesichts dessen kommt es zur Konkurrenz nicht nur der Leistungserbringer untereinander, sondern auch der Leistungserbringer der Integrationsgemeinschaft mit bereits bestehenden Kooperationsgemeinschaften von Leistungserbringern, die in der Konkurrenz der Gesellschaftsrechte entweder lose verbunden oder aber rechtsförmlich ,integriert" sind. Weiterhin konkurrieren Gemeinschaften mit Gemeinschaften und alle in diesem Sinne geeigneten Vertragspartner mit Leistungserbringerstatus mit Manage-

761 Sichert/Walser, Management of Chronic Diseases in the Netherlands and in Germany, European Journal of Social Security, Vol. 9 (2007), S. 243 (261 ff.).

762 Vgl. Jasper/Sundmacher, Wettbewerb und Kooperation als Koordinationsverfahren für die Integrierte Versorgung, SF 2005, S. 53 ff. 
mentgesellschaften, die - ohne Leistungserbringer zu sein - selbst eine Integrierte Versorgung 763 anbieten können.

Soweit schließlich Krankenhäuser um den Abschluß von Selektiv- und insbesondere Integrationsverträgen konkurrieren, wird diese Konkurrenz umso größer, je eher auch unter räumlichen Gesichtspunkten Alternativen für die Krankenkassen bestehen, d.h. insbesondere in den Städten. Dennoch bliebt es unter regionalen Aspekten, angesichts der Kostenintensität der Krankenhausbehandlung sowie der gewichtigen Position an den bereichsspezifischen Schnittstellen der Versorgung bei einer herausgehobenen Position der Krankenhäuser.

Das KHRG hat keine Verträge etwa vom Typus der „,besonderen stationären Versorgung" (§ 73c SGB V vergleichbar) etabliert bzw. Elektivleistungen dem Selektivvertrag überantwortet. In diesem Bereich könnten daher Verträge zur Integrationsversorgung weiter an Bedeutung zunehmen. Allgemein werden die materiellen bzw. normativen Grenzen des Vertragsgeschehens v.a. durch den jeweiligen Typus selbst vorgegeben, vgl. etwa $\S 140 \mathrm{~b}$ Abs. 4 SGB V. Dessen ungeachtet besteht streng genommen zwar auch im Sozialversicherungsrecht als einer Materie des Öffentlichen Rechts und nach Maßgabe der $\S \S 53$ ff. SGB X (vgl. auch $\S 37$ SGB I) kein Vertragstypenzwang. ${ }^{764}$ Indes ist die Frage nach einem Entgegenstehen öffentlich-rechtlicher Vorschriften im Sinne materieller Grenzen insoweit besonders streng zu beäugen. Eine Abweichung insbesondere von den im Regime der $\S \S 108$ ff. SGB V zentral verhafteten $\S 109$ SGB $\mathrm{V}$ dürfte insoweit nicht in Betracht kommen.

\subsubsection{2. „DMP-Verträge“}

Ein veritables Beispiel für die Konkurrenz und Kombination der Selektivvertragstypen bilden Verträge über Disease Management Programme. Mit Blick auf die Beteiligung der Krankenhäuser und hinsichtlich ihrer Relevanz für die Wahl der Krankenkasse wurden DMP-Verträge bereits illustriert. ${ }^{765} \mathrm{Gem}$. § 116b Abs. 1 SGB V können seitens der Krankenkassen mit Krankenhäusern, die an der Durchführung mit DMP teilnehmen, Verträge über ambulante ärztliche Versorgung geschlossen werden, „soweit die Anforderungen an die ambulante Leistungserbringung in den Verträgen zu den strukturierten Behandlungsprogrammen dies erfordern“. Dieser mitunter vernachlässigte Passus deutet auf eine Unterscheidung zwischen DMP-Vertrag und Durchführungsvertrag zur Realisierung der DMP. Eine solche Trennung ist gleichwohl nicht zwingend, wohnt Verträgen nach $\S 116 \mathrm{~b}$ Abs. 1 SGB V doch ein hinreichendes Integrationspotential inne. Unbeschadet dessen ist möglich, einen Integrationsvertrag gem. § 140a ff. SGB V zur Implementierung der DMP zu schließen, und auch Modellvorhaben nach $\S 63 \mathrm{ff}$. SGB V

$763 \S 140 \mathrm{~b}$ Abs. 1 Nr. 4 SGB V; deren Betätigungsfeld wurde mit der Einführung des Vertragspartnerschaftsstatus in $\S 73 \mathrm{c}$ SGB V für die besondere ambulante Versorgung maßgeblich erweitert.

764 S. zur Zulässigkeit von Rabattverträgen bereits vor Inkrafttreten des § 130a SGB V BVerfG, Beschl. v. 13.9.2005, SozR 4-2500, § 266 Nr. 9, S. 147 (164 Rn. 69).

765 Vgl. oben II.2.1.2. 
sind ein gangbarer Weg, ebenso wie jenseits des engeren Kreises der Selektivverträge Strukturverträge gem. § 73a SGB V sowie dreiseitige Verträge nach § 115 SGB V. All diese Vertragsformen sind im Hinblick auf die Umsetzung von DMP-Verträgen tatsächlich vorzufinden, ${ }^{766}$ wobei für die Krankenhäuser und mit Blick auf den Selektivvertragsbereich die erstgenannten drei in Betracht kommen.

Die Zulassung der DMP beim BVA setzt voraus, daß sowohl die Programme als auch „die zu ihrer Durchführung geschlossenen Verträge die in der RSAV genannten Voraussetzungen erfüllen“, § 137g Abs. 1 S. 1 SGB V. Bezieht man diese Vorschrift sowie die Erwähnung ,vertraglich vereinbarter Programme“ gem. § 137f Abs. 5 S. 2 SGB V in die Betrachtung mit ein, könnte man davon ausgehen, daß auch hier die Vertragsgrundlage zu suchen ist. ${ }^{767}$ Letzteres allerdings erscheint wenig überzeugend, denn in diesem Falle sind aus vertragsrechtlicher Sicht überhaupt keine Anforderungen normiert, nicht einmal hinsichtlich der Vertragspartner. Die bessere Lösung wäre danach, entweder auf zahlreich vorhandene Vertragsgrundlagen zurückzugreifen, welche allerdings mit Blick auf die stationären Belange bis auf § 140a ff. SGB V extensiv interpretiert werden müssten, oder aber in den Bestimmungen der $\S \S 137 \mathrm{f}$ und $137 \mathrm{~g}$ SGB V eine Erlaubnis der Handlungsform DMP-Vertrag zu sehen. Beides ist auch mit Blick auf $\S 53 \mathrm{ff}$. SGB X vertretbar.

Sowohl Verträge nach $\S \S 63 \mathrm{ff}$. als auch gem. $\S 116 \mathrm{~b}$ und $\S \S 140 \mathrm{a}$ ff. SGB V können einen Gestaltungsspielraum hinsichtlich der Vergütung nutzen. Im Bereich der Modellvorhaben und der IV kommt es zur Bereinigung der Gesamtvergütung. Mit Blick auf den ebenfalls existenten, gleichwohl ungelösten „Koordinationsbedarf gegenüber dem Kollektivsystem“ erfuhr demgegenüber $§ 116$ b SGB V schon vor dem GKV-WSG Kritik. ${ }^{768}$

\subsubsection{Verträge zur Integrierten Versorgung}

Im Selektivvertragsbereich nehmen IV-Verträge eine besondere Position ein, 769 v.a. im Zeichen der Möglichkeit einer Beteiligung auch der Krankenhäuser. Aufgrund ihrer Managementkapazitäten führen die Krankenhäuser die Integrationsgemeinschaft auf Leistungserbringerseite oftmals an.

Bei den zum 31.12.2008 registrierten 6.183 Versorgungsverträgen 770 halten die als Vertragspartner direkt beteiligten Krankenhäuser auf Seiten der Leistungserbringer ins-

766 Ausf. Sichert/Walser, Management of Chronic Diseases in the Netherlands and in Germany, European Journal of Social Security, Vol. 9 (2007), S. 243 (261 ff.).

767 So Cassel u.a., Weiterentwicklung des Vertragswettbewerbs in der gesetzlichen Krankenversicherung (2006), S. 56 f., wobei $§ 137 f$ Abs. 6 S. 1 SGB V hier ggf. eher geeignet erschiene.

768 Cassel, a.a.O., S. 55.

769 S. zum mediznisch-inhaltlichen Zuschnitt der Versorgungskonzepte oben II.2.1.2.

770 www.bqs-register140d.de; Zählung der gemeldeten Verträge einfach je Kasse. Die Zahl der Versicherten lag bei rund 4 Mio., das Vergütungsvolumen bei $€ 800$ Mio. Die Zahl der Verträge stieg stetig: 2005 (1.913), 2006 (3.309), 2007 (5.069); Stichtag, auf für die folgenden Angaben, ist der 31.12. des Bezugsjahres. 
gesamt gesehen den größten Anteil bei den Vertragspartnerkombinationen. In der Abbildung der direkten Vertragspartnerkombinationen, bei welcher der Ausweis der ,alleinigen“ Vertragspartnerschaft auf Leistungserbringerseite die Stellung als „Generalübernehmer" kennzeichnet, der seinerseits Unterverträge abschließt, ergibt sich folgendes: Die Krankenhäuser sind zu 16\% (= 990 Häuser) im Alleinanteil ${ }^{771}$ ausgewiesen, agieren darüber hinaus in Kooperation mit Niedergelassenen Ärzten $(18,7 \%)^{772}$, mit Rehabilitationseinrichtungen $(10,7 \%)^{773}$ sowie zu 2,9\%774 in Kombination mit beiden. Ihnen stehen die Krankenkassen auf Kostenträgerseite gegenüber.

Die Zahlen deuten - im Spiegel der Vorjahre - an, daß das Krankenhaus seine „Vormachtstellung“ als "Generalunternehmer"775, der die IV auf Leistungserbringerseite organisiert und Unterverträge schließt, relativ gesehen eingebüßt hat. Das mag zum einen darin liegen, daß die Kapazität zur Vertragsgestaltung mittlerweile auch andere Leistungserbringer mitbringen, insbesondere Gemeinschaften der Leistungserbringer (vgl. § 140b Abs. 1 Nr. 6 SGB V) bzw. zunehmend Managementgesellschaften (§ 140b Abs. 1 Nr. 4 SGB V). Gleichzeitig aber ist der „Einstieg“ anderer Leistungserbringer relativ zu sehen und v.a. auf deren Gesamtzahl zu beziehen. Denn viele der mehr als 2.000 Krankenhäuser waren frühzeitig an Verträgen beteiligt, die in der Idealvorstellung und einer die Sektoren vereinfachend beschreibenden Betrachtungsweise die Integration von „stationär und ambulant“ ermöglichten. Dieser Schwerpunktsetzung entspricht es im übrigen, daß die bis Ende 2008 gewährte Anschubfinanzierung für die ab dem 1.4.2007 geschlossenen Verträge auf die Finanzierung der Leistungen der Krankenhäuser und der Vertragsärzte begrenzt wurde, § 140d Abs. 1 S. 2 SGB V. Gleichzeitig wurden hinsichtlich der Krankenhausleistungen nur die über die Regelversorgung hinaus vereinbarten aus der Anschubfinanzierung vergütet, vgl. § 140d Abs. 4 SGB V. Nicht zu verkennen ist indes, daß nach $\S 140 \mathrm{~b}$ Abs. 4 S. 1 KHG auch von den Bestimmungen des KHG und des KHEntgG abgewichen werden darf.

Mit dem Wegfall der Anschubfinanzierung ist davon auszugehen, daß § 140d Abs. 4 SGB V für Neuverträge seine Wirkung verliert und entsprechend der Begründung zum VÄndG ,nach dem Ablauf der Anschubfinanzierung... die Leistungen vollständig aus dem Krankenhausbudget ausgegliedert" werden. ${ }^{776}$ (Erst) mit der Beschlussempfehlung des 14. AfG erfolgte in $\S 4$ Abs. 1 S. 2 Alt. 4 KHEntgG schließlich die insoweit konsequente „Klarstellung, daß die nach $\S 140$ c SGB V zu finanzierenden Leistungen

771 Gegenüber 2005 (23\%), $2006(20 \%)$ und 2007 (17,5\%).

772 Gegenüber 2005 (17\%), $2006(17,6 \%)$ und $2007(19,9 \%)$.

773 Gegenüber 2005 (20\%), 2006 (16\%) und $2007(12,4 \%)$.

774 Gegenüber 2005 (4\%), 2006 (3,8\%) und 2007 (3,3\%).

775 Es ist Ausdruck der Flexibilität des Gestaltungsfreiheit vermittelnden Rechtsregimes für IVVerträge, daß ein IV-Vertrag auch durch nur einen Partner auf Leistungserbringerseite („Generalunternehmer", s.o.) zustande kommt. $§ 140 \mathrm{~b}$ Abs. $1 \mathrm{Nr}$. 4 SGB V verdeutlicht dies. 
der integrieren Versorgung nicht Bestandteil des Erlösbudgets nach dem Krankenhausentgeltgesetz sind“. 777

Nach den Grundsätzen zulässiger Beteiligungen und Integrationsleistungen können auch Krankenhäuser zweier Fachrichtungen (etwa. Onkologie und Strahlentherapie) „interdisziplinär-fachübergreifend“ miteinander kooperieren. ${ }^{778}$ Praxiskliniken können eigenständig als Vertragspartner auftreten. ${ }^{779}$ Besondere Bedeutung erlangen ferner Verträge an der Schnittstelle zwischen Akutversorgung und medizinischen Leistungen der Rehabilitation. Wie auch $\S 112$ Abs. 2 S. 1 Nr. 5 SGB V verdeutlicht, ist die Rehabilitation - soweit nicht die Frühphase als integraler (und damit nicht gesondert ,,integrationsfähiger") Bestandteil der Akutversorgung betroffen ist - nicht Bestandteil der Krankenhausbehandlung. ${ }^{780}$ Das $B S G$ hat entschieden: ${ }^{781}$

\begin{abstract}
„Eine integrierte Versorgung kann... durch Verknüpfung von stationärer Akutbehandlung und stationärer Rehabilitation erreicht werden, weil diese Leistungssektoren in der traditionellen Versorgung inhaltlich und institutionell typischerweise getrennt sind. Diese Trennung ist in den bisherigen Strukturen immer dann gegeben, wenn es sich um unterschiedliche Einrichtungen handelt... Aber auch in den selteneren Fällen, in denen ein Träger übergreifend sowohl ein Krankenhaus als auch eine Rehabilitationseinrichtung betreibt, können Verknüpfungen... eine verschiedene Leistungssektoren übergreifende Versorgung... bilden. Denn Art und Inhalt der jeweils zu erbringenden Leistungen sind im Krankenhaus einerseits und in der Rehabilitationseinrichtung andererseits unterschiedlich.“... „Unzureichend für eine integrierte Versorgung ist es dagegen, wenn innerhalb eines Krankenhauses nur die stationär und die ambulant erbrachten ärztlichen Behandlungen miteinander verknüpft werden... 782 .
\end{abstract}

Im Jahre 2005 hatten die meisten der an der Umfrage 2006 des DKI teilnehmenden 341 Krankenhäuser, nämlich 53,5\%, einen Vertrag abgeschlossen. ${ }^{783}$ Der Anteil der Krankenhäuser mit mehreren Verträgen hat um 14\% gegenüber dem Jahre 2004 zugenommen; die an der IV teilnehmenden Häuser verfügten über durchschnittlich 2,5 Integrationsverträge. ${ }^{784}$ Der mit $50 \%$ wichtigste Leistungsbereich ist dabei die endoprothetische Versorgung. ${ }^{785}$ Die durchschnittlichen Erlöse der an der IV teilnehmen-

777 BT-Drs. 16/11429 (elektron. Vorabfassung), S. 58.

778 Quaas, in: ders./Zuck, Medizinrecht (2008), § 11 Rn. 76 (S. 188).

$779 \S 140$ b Abs. 1 Nr. 7 SGB V.

780 Vgl., auch unter dem Aspekt der Rehabilitation im Ausland, Sichert, Gemeinschaftsrechtliche Vorgaben für Integrierte Versorgung, GuS 2007, S. 28 (29).

781 BSG, Urteile. v. 6.2.2008, B 6 KA 5/07 R und B 6 KA 6/07 R, jew. Rn. 23.

782 A.a.O., ,es sei denn dass die Verzahnung deutlich über das Maß hinausgeht, das normalerweise in der traditionellen Versorgung besteht... etwa bei Krankheitsbildern, bei denen ein Wechsel zwischen der zB in der Institutsambulanz regelmäßig stattfindenden ambulanten und den zwischenzeitlich erforderlichen stationären Behandlungen typischerweise gehäuft vorkommt ... (möglicherweise bei psychiatrischen Patienten)“.

783 DKI, Krankenhaus Barometer, Umfrage 2006, S. 53.

784 A.a.O.

785 A.a.O., S. 54. 
den Krankenhäuser im Jahre 2005 lagen bei $€ 388.000 .{ }^{786}$ Positiven Einschätzungen zufolge beträgt das Einsparpotential durch IV-Verträge 5\%. ${ }^{787}$ Dem durchschnittlichen Erlösvolumen standen Rechnungskürzungen bei den teilnehmenden Krankenhäusern von im Durchschnitt $€ 235.000$ gegenüber. ${ }^{788}$

Von den großen „Playern“ einmal abgesehen hält sich das Einnahmepotential offenbar in Grenzen. Restriktionen sind mutmaßlich ebenso auf die Vergütungsfähigkeit allein der Zusatz-, Integrations- und Dokumentationsleistungen (bis 2008) zurückzuführen wie darauf, daß der "Dispens“ von der Arzneimittelpreisverordnung 789 in den an der IV beteiligten ambulanten Bereich nicht transferiert werden kann. Jedenfalls aber musste das Krankenhaus vormals darauf bedacht sein, Rechnungskürzungen und andere „Strukturnachteile“ zu kompensieren.

Nach dem Wegfall der Anschubfinanzierung einschließlich deren Refinanzierung über (Krankenhaus-) Rechnungskürzungen sowie im Kontext qualitativer Fortentwicklung der IV-Vertragsmodelle stellt sich das Integrations- und Einsparpotential ggf. positiver dar. Möglicherweise lassen die Vertragspartnerstrukturen auch Rückschlüsse auf differenzierende Weiterentwicklungen zu, wenn man sich vor Augen führt, daß für das Jahr 2005, denen die umfrageerfassten Erlös-Daten zuzuordnen sind, u.U. noch Verträge dominierten, die allein klassische Schnittstellen (,stationärer Bereich - Reha“) (punktuell) erfassten. Da das KHRG nicht dazu führte, frei bzw. planbar wählbare (Elekiv-) Leistungen generell dem Selektivvertragswettbewerbs zu überantworten, könnte möglicherweise ein Teil des Weges über die Integration beschritten werden.

Kein Wettbewerbsvorteil schließlich liegt darin, über Krankenhausapotheken (§ 14 ApoG) 790 eine günstige Arzneimittelversorgung mit Blick darauf organisieren zu wollen, daß Krankenhausapotheken unbeschadet ihrer Qualifikation als Funktionseinheiten bzw. Leistungserbringer 791 nach $\S 1$ Abs. 3 Nr. 1 nicht an die Arzneimittelpreisverordnung (AMPreisV) gebunden sind. ${ }^{792}$ Denn werden Krankenhausapotheken in die IV

786 DKI (Hrsg.), Krankenhaus Barometer, Umfrage 2006, S. 55 f. Bezogen auf das Instrument der IV stehen diesen Rechnungskürzungen (bei allen Häusern) gegenüber, die die teilnehmenden jedoch „überkompensieren“ können, s. unter I.2.2.4.

787 Fellgiebel/Schanz/Huwer, IV-Vertrag zur Frühversorgung von Demenzpatienten mit der Barmer Ersatzkasse, das Krankenhaus 2007, S. 318.

788 A.a.O., S. 56.

789 Vgl. oben I. 2.1.3.2.

790 Die Regelungen in $\S 14$ ApoG über den Betrieb von Krankenhausapotheken sind im Hinblick auf Art. 28 und 30 EGV nicht zu beanstanden; ,,insbesondere ist es aus Gründen des Gesundheitsschutzes nicht geboten, die Versorgung eines Krankenhauses auch durch nicht in dessen Nähe liegende Apotheke zu ermöglichen“, EuGH, Urt. v. 11.9.2008, Rs. 141/07, GesR 2009, S. 99.

791 Siehe zur Einordnung der Krankenhausapotheken als Leistungserbringer Wigge, Medizinische Versorgungszentren nach dem GMG, MedR 2004, S. 123 (132). Vgl. demgegenüber Grau, in: Bohle, Vertragsgestaltung in der integrierten Versorgung (2005), S. 55 (56). Nach § 129a SGB V ist es den Krankenhausapotheken möglich, ambulante Patienten der Trägerkrankenhäuser direkt zu versorgen, vgl. auch Ballast, Die Ersatzkasse 2004, S. 221 (222).

$792 \S 1$ Abs. 3 Nr. 1 AMPreisV. I.ü. aber vollzieht $\S 14$ des ApoG in Abs. 7 S. 3 die erweiterten Behandlungsmöglichkeiten der Krankenhäuser nach, soweit Arzneimittel von Krankenhaus- oder 
eingebunden, darf die Vertragsgestaltung mangels Befugnis zum Dispens 793 vom Apothekengesetz 794 nicht dazu führen, daß die Versorgungstätigkeit der Krankenhausapotheken allgemein auf den ambulanten Bereich erstreckt wird. ${ }^{795}$

\subsubsection{Die Krankenkassen als Unternehmen}

Daß und inwieweit Schutz gegen marktmissbräuchliches Verhalten der Krankenkassen auch beim krankenhausspezifischen Versorgungsmanagement nach $\S 69$ S. 2 SGB V i.V.m. §§ 19-21 GWB (entsprechend) besteht, wurde bereits dargelegt, ebenso die Bindung der Krankenhäuser an das Kartellrecht (einschließlich der Fusionskontrolle), sollte es nicht ausnahmsweise um die spezifische Erfüllung versorgungsrechtlicher Vertragsbeziehungen gehen. Auch die Bindung der Krankenhäuser an Art. 81 und 82 EGV ist unzweifelhaft.

Nachfolgend stehen die Bindungen der gesetzlichen Krankenkassen an das europäische Recht im Vordergrund, soweit sie über Spielräume im Selektivertragsbereich verfügen. Die wegen ihrer „Staatsnähe“ bzw. der Berücksichtung und Erfüllung des öffentlichen und sozialen Interesses diskutierten Anwendungsbegrenzungen erfassen die PKV-Unternehmen nicht. Für die Beurteilung der Wettbewerbswidrigkeit der Aktivitäten der gesetzlichen Krankenkassen nach Art. 81 f. EGV kommt es entscheidend darauf an, ob diese im Rahmen ihrer Tätigkeit den im relevanten Gesundheitsmarkt gemeinschaftsautonom auszufüllenden Begriff des Unternehmens und damit den persönlichen Anwendungsbereich des europäischen Kartellrechts erfüllen. Nach hier vertretener Auffassung handeln die Krankenkassen in Abgrenzung zur Entscheidung des $E u G H$ in Sachen AOK-Bundesverband 796 hinsichtlich besonderer Selektivvertragsgestaltungen dann als Unternehmen, wenn ihr Tätigwerden in der solidarischen Wettbewerbsordnung mit erheblichen Verhaltensspielräumen entsprechend einer marktmäßigen Ausrichtung folgt, den diese Ordnung weithin selbst vorgegeben hat:

Die Grundüberlegung lautet, inwiefern die Krankenkassen nach dem maßgeblichen funktionalen Unternehmensbegriff im Sinne einer wirtschaftlichen Tätigkeit „Güter

krankenhausversorgenden Apotheken auch im Rahmen der ambulanten Behandlung in Krankenhäusern abgegeben werden dürfen.

793 Grau/Dierks, Die Versorgungsbereiche von öffentlicher und Krankenhausapotheke in der Integrierten Versorgung, A\&R 2005, S. 106 (107 Fn. 8).

794 Vgl. insbesondere $\S 14$ Abs. 4 ApoG.

795 Grau/Dierks, Die Versorgungsbereiche von öffentlicher und Krankenhausapotheke in der Integrierten Versorgung, A\&R 2005, S. 106 (107 Fn. 8); Koller, Die Abgabebefugnis von Krankenhausapotheken in der ambulanten Versorgung, ApoR 2006, S. 20 (22); Grau, in: Bohle, Vertragsgestaltung in der integrierten Versorgung (2005), S. $58 \mathrm{ff}$.

796 EuGH, Urt. v. 16.3.2004, verb. Rs. en C-264/01, C-306/01, C-354/01 und C-355/01 (AOK Bundesverband u.a.), Slg. 2004, I-2493, Rn. 58. 
oder Dienstleistungen auf einem bestimmten Markt anzubieten“797 haben.798 Ein „echter" „Angebotsmarkt" ist mit Blick auf die sozialrechtlichen Leistungsbeziehungen traditionell schwer vorstellbar, gerade in Bezug auf das „Angebot an Gesundheitsleistungen“. Die jüngere Rspr. (v.a. in Sachen FENIN) ${ }^{799}$ geht davon aus, ,daß bei der Beurteilung des Wesens der Einkaufstätigkeit der Kauf eines Erzeugnisses nicht von dessen späterer Verwendung zu trennen ist und daß der wirtschaftliche oder nichtwirtschaftliche Charakter der späteren Verwendung des erworbenen Erzeugnisses zwangsläufig den Charakter der Einkaufstätigkeit bestimmt. " 800 Hintergrund dessen ist die stets in relativer Betrachtungsweise zu beurteilende wirtschaftliche Zweckerfüllung und Ausrichtung einer konkret zuzuordnenden unternehmerischen Tätigkeit in Abgrenzung zur Wahrnehmung einer „Aufgabe mit ausschließlich sozialem Charakter" 801 entsprechend dem „Grundsatz der nationalen Solidarität“, gemäß der Ausübung „ohne Gewinnzweck“ sowie der Leistungserbringung unabhängig von der Höhe der Beiträge. 802 Gleichwohl wird die Möglichkeit des Vorliegens unterschiedlicher „Solidaritätsgrade“ 803 konstatiert und angemahnt, im Wege der Akzessorietätsbetrachtung nicht den Blick dafür zu verlieren, ob das Einkaufsgut auf dem „,bestimmten“ oder - allein als „Einsatzgut“ auf einem ,anderen Markt“ angeboten werde. ${ }^{804}$ Nach alledem würde eine streng akzesso-

797 EuGH, Urt. v. 22.1.2002, Rs. C-218/90 (CISAL), Slg. 2002, I-691, Rn. 23; EuG, Urt. v. 4.3.2003, Rs. T-319/99 (FENIN), Slg. 2003, II-357, Rn. 35; EuGH, Urt. v. 11.7.2006, Rs. C-205/03 (FENIN), Slg. 2006, I-6295, Rn. 25.

798 Im Übrigen wird die solchermaßen ,wirtschaftlich tätig werdende Einheit [,] unabhängig von ihrer Rechtsform und Finanzierungsart“" erfaßt; EuGH, Urt. v. 23.4.1991, Rs. C-41/90 (Höfner und Elser), Slg. 1991, I-1979, Rn. 21; s. ferner EuGH, Urt. v. 22.1.2002, Rs. C-218/90 (CISAL), Slg. 2002, I691, Rn. 22.

799 EuG, Urt. v. 4.3.2003, Rs. T-319/99 (FENIN), Slg. 2003, II-357, durch den Gerichtshof bestätigt, s. EuGH, Urt. v. 11.7.2006, Rs. C-205/03 (FENIN), Slg. 2006, I-6295.

800 EuGH, Urt. v. 11.7.2006, Rs. C-205/03 (FENIN), Slg. 2006, I-6295, Rn. 26; EuG, Urt. v. 4.3.2003, Rs. T-319/99 (FENIN), Slg. 2003, II-357, Rn. 36. Die gesundheitsverwaltenden Einrichtungen handelten ,,auch dann nicht als Unternehmen, wenn sie medizinischen Bedarf bei anderen Unternehmen einkaufen, um den diesem nationalen Gesundheitssystem Angeschlossenen kostenlose medizinische Leistungen zu erbringen“; EuGH, Urt. v. 11.7.2006, Rs. C-205/03 (FENIN), Slg. 2006, I-6295 Ls. 2, Rn. $38 \mathrm{ff}$.

801 EuGH, Urt. v. 17.2.1993, Rs. C-159/91 und C-160/91 (Poucet und Pistre), Slg. 1993, I-637, Rn. 18.

802 A.a.O. Demgegenüber agieren Sozialversicherungsträger als Unternehmen, wenn sie, wie der unternehmerisch tätige Träger einer Zusatzrentenversicherung, eine dieser Voraussetzungen nicht erfüllen, EuGH, Urt. v. 16.11.1995, Rs. C-244/94 (Fédération française des sociétés d'assurance), Slg. 1995, I-4013, Rn. 18 ff. Dies ist vor allem der Fall bei freiwilliger Mitgliedschaft, der Erhebung der Beiträge entsprechend dem Kapitalisierungsprinzip und der daran anknüpfenden Gewährung von Leistungen, a.a.O. Rn. 17.

803 A.a.O., Rn. 32.

804 Die Bezugnahme auf das Akzessorietätserfordernis in Sachen $A O K$-Bundesverband durch GA $J a-$ cobs (Schlussanträge, verb. Rs. en C-264/01, C-306/01, C-354/01 und C-355/01 [AOK Bundesverband u.a.], Slg. 2004, I-2493, Rn. 45 f.) bedeutet keine Absage an den relativen Unternehmensbegriff. Der Fall unterscheide sich von dem in der Sache FENIN insofern, als „die Krankenkassen auf einem anderen Markt tätig sind und einen Beitrag zum Erwerb von Arzneimitteln als Teil ihrer Tätigkeit auf diesem Markt leisten“; a.a.O. Rn. 46 und Fn. 18. Dennoch wird die Angebotstätigkeit der 
rische Betrachtung nach dem Muster des „Einkaufs von Integrationsleistungen“ und des „Angebots ebendieser“ nicht zur Unternehmenseigenschaft der Krankenkassen führen. Es käme bestenfalls zur unternehmerischen Tätigkeit im Wege des Betreibens von Eigeneinrichtungen und der Beteiligung an Gesellschaften von Leistungserbringern oder Managementgesellschaften. ${ }^{805}$ Das Akzessorietätsgerüst ist hinsichtlich der Frage, wann die Krankenkassen im sozialrechtlichen Leistungsgefüge funktional und zweckgerichtet wirtschaftlich agieren, eine mit Blick auf den Tatbestand überschießende und „Solidaritätsgraden“ nicht gerecht werdende Einschränkung.

Dennoch stellen die Krankenkassen Leistungen ,zur Verfügung““806 Gerade im Beziehungsgeflecht moderner Vertrags- und Versorgungsformen erscheinen die Leistungen daher als solche „der jeweiligen Kassen“. In spezifischen Bereichen ist eine Leistungsdiversifikation auszumachen, mit der eine Prämiendiversifikation korreliert.

Im Bereich der IV unterscheidet sich die angebotene Leistung oftmals von der Regelversorgung: Hier sind etwa Präventionsleistungen, Sonderberatungen, die Behandlung nach Behandlungsleitpfaden oder die konkrete Behandlungsabstimmung im Rahmen von DMP zu nennen. Es geht um Rationalisierungsgewinne, darüber hinaus um die Gewinnung - oder das Halten - von Versicherten, zu deren Versorgung die Krankenkassen gem. §§ 266 und 270 SGB V Zuweisungen aus dem Gesundheitsfonds erhalten. Neben den Qualitätsanreiz für Versicherte tritt die besondere Tarifgestaltung (vgl. § 53 Abs. 3 S. 1 SGB V), speziell i.V.m. Prämienzahlungen (an den Versicherten) oder Zuzahlungsermäßigungen (Abs. 3 S. 2). Auch Solidarität herrscht hier nur eingeschränkt, denn unbeschadet der generellen Relevanz für die GKV-weite Standardisierung der Leistungsausgaben bleibt den Kassen der erwirtschaftete Vorteil erhalten. „Unternehmerisches“" Wirtschaften trägt insofern dazu bei, ob etwa ein Zusatzbeitrag i.S.d. § 242 Abs. 1 SGB V vermieden oder eine Prämie nach Abs. 2 der Vorschrift ausgeschüttet werden kann. Dies alles vollzieht sich - für manche Kassen - vor dem bedrohlichen Hintergrund einer möglichen Insolvenz. ${ }^{807}$

Soweit im Zeichen selektiver Modelle wie der IV (v.a. bei zusätzlichen Leistungen) oder Rabattvereinbarungen (§ 140a [Abs. 1 S. 5. i.V.m.] § 130a Abs. 8 SGB V) Leistungs- und Prämiendiversifikation (§31 Abs. 2 S. 2, §53 Abs. 3, 4 SGB V) miteinander korrelieren, spricht einiges dafür, daß die Krankenkassen hier funktional als Unternehmen agieren und u.a. hinsichtlich ihres Verhaltens den Krankenhäusern gegenüber der Kontrolle des europäischen Kartellrechts nach Art. 81 und 82 EGV unterworfen sind.

Krankenkassen, nicht zuletzt weil sie von der Kerntätigkeit nicht näher zu trennen sei, nicht näher beschrieben, die Krankenkassen seien nämlich auch auf dem so bezeichneten ,anderen“ Markt tätig, für den die eingekauften Waren oder Dienstleistungen ein Einsatzgut darstellten.

805 Kamann/Gey, Wettbewerbsrecht im deutschen Gesundheitswesen, PharmR 2006, S. 255; 291 (293).

$806 \S 2$ Abs. 1 SGB V.

807 Vgl. v.a. § 171 b ff. SGB V i.d.F. des GKV-OrgWG. 
Gleichwohl ist an eine Bereichsausnahme gem. Art. 86 Abs. 2 EGV zu denken, denn die Krankenkassen stellen Versicherten die im SGB V genannten Leistungen zur Verfügung, ${ }^{808}$ und dies dient der Versorgung zur Aufgabenerfüllung der Krankenversicherung als Solidargemeinschaft. ${ }^{809}$ Zudem mag man davon ausgehen, daß selektivvertragliches Handeln nach den jüngsten Reformen „,zur Aufgabenerfüllung erforderlich“ ist. 810 Das bedeutet aber nicht, daß bei Wahrnehmung und Ausübung der eingeräumten Gestaltungsmöglichkeiten jene Handlungsweisen im Vordergrund stehen müssten, die Wettbewerb verfälschen (nur dann greift Kartellrecht ein). Angesichts der Bandbreite zulässiger Handlungsweisen kann auch nicht von der Verhältnismäßigkeit einer jeden Beeinträchtigung des Wettbewerbs unter Rechtfertigung des (Vertrags)Handelns durch das allgemeine Interesse 811 ausgegangen werden.

\subsubsection{Ein neuer „,bestimmter Markt“ innerhalb des GKV-Systems?}

Die vorstehende Betrachtungsweise führt über die bisher restriktive Anschauung hinaus, Marktmechanismen könnten sich im GKV-System lediglich dort etablieren, wo wettbewerbliche Strukturen über die Freiwilligkeit der Versicherung vermittelt werden. 812 Die (tradierte) Auffassung erblickt Wahlmöglichkeiten auf der (Versicherungs-) Angebotsseite tendenziell allein darin, daß dem „Konsumenten“ grundsätzlich der gesamte Markt einschließlich der Entscheidung über das „Grundverhältnis“ der GKVoder PKV-Zugehörigkeit offen steht. Damit aber wird die Bewertung (und Kontrolle) wettbewerbsorientierter Handlungsspielräume an Bedingungen geknüpft, die den funktionalen Unternehmensbegriff auf eine bereichsspezifische Wirtschaftstätigkeit der höchsten in diesem Bereich vorzufindenden Marktstufe reduzieren und so den „,bestimmten Markt" im Sinne der Rechtsprechung des $E u G H$ äußerst restriktiv definieren. Auf diese Weise werden marktorientierte Wahlfreiheiten im Bereich der Prämien- und Leistungsdiversifikation und deren Verknüpfung außer Acht gelassen und verkannt, daß in diesen Bereichen die Solidaritätsstrukturen 813 weitaus weniger stark ausgeprägt sind:

$808 \S 2$ Abs. 1 SGB V; s.a. GA Jacobs, Schlußanträge in den verb. Rsen. C-264/01, C-306/01, C-354/01 und C-355/01 (AOK Bundesverband u.a.), Slg. 2004, I-2493 Rn. 87.

809 Nach dem Urteil in der Sache Albany etwa gehört zu den Dienstleistungen von allgemeinem wirtschaftlichem Interesse auch die Erfüllung einer wesentlichen sozialen Funktion, wie dem entscheidungsgegenständlichen Zusatzrentensystem. Urt. v. 21.9.1999, Rs. C-67/96 (Albany), Slg. 1999, I5751 Rn. 103 ff., 105.

810 S. zu diesem Kriterium EuGH, Urt. v. 23.10.1997, Rs. C-157/94 (Kommission/Niederlande), Slg. 1997, I-5699 Rn. 53; Urt. v. 21.9.1999, Rs. C-67/96 (Albany), Slg. 1999, I-5751 Rn. 107.

811 Siehe Grill, in: Lenz/Borchardt, EUV/EGV, 4. Aufl. (2006), Art. 81 EGV Rn. 29. Dies mag als eine Konkretisierung der Erforderlichkeitsprüfung im Sinne des Verhältnismäßigkeitsprinzips gesehen werden, vgl. Becker, in: in: Schnapp/Wigge, Handbuch des Vertragsarztrechts, 2. Aufl. (2006), § 25 Rn. 60.

812 Vgl. z.B. Roth, Kartellrechtliche Aspekte der Gesundheitsreform nach deutschem und europäischem Recht, GRUR 2007, S. 645 (658 f.)

813 Vgl. auch Schmidt, GKV-WSG: die Relativierung klassischer Sozialversicherungsmerkmale in der GKV durch veränderte Beitragsbemessung und Wahltarife, GesR 2007, S. 295 ff. 
der Kranke wird faktisch keine Selbstbehalt-Tarife wählen können, wohl aber - und dies bedeutet Wettbewerb um Kranke (!) - DMP-Programme; sämtliche Einsparungen dienen (nur) mittelbar der finanziellen Konsolidierung des GKV-Gesamtsystems. Insofern ist nach hier vertretener Auffassung auch der wirtschaftlich determinierte Markt um Tarif- und Angebots- sowie absatz- und kontrollprivilegierte Versorgungsstrukturen ein „bestimmter Markt“ im Sinne der Rspr. des EuGH.

\subsubsection{Pflicht zur Ausschreibung von Kooperationsverträgen}

Unter regulatorischen und ökonomischen Gesichtspunkten - v.a. mit Blick auf Transaktionskosten der Marktteilnehmer - stellt sich alsdann die Frage, ob die Krankenkassen Versorgungsverträge insbesondere zur Verzahnung des ambulanten und stationären Sektors ausschreiben müssen. Eine damit bereits praktisch verbundene Konsequenz ist die, ob die Leistungserbringer ihrerseits auf die Kassen zugehen und ,Angebote" machen können, was für die Managementgesellschaft auch $\S 140 \mathrm{~b}$ Abs. 1 Nr. 4 SGB V suggeriert. Wie bereits dargelegt ${ }^{814}$ geht es im Gefolge der Änderung des $\S 69$ SGB V durch das GKV-OrgWG im wesentlichen „nur“ noch darum, ob es sich bei den Vergaben um öffentliche Aufträge handelt.

„Diese Frage“, so die Gesetzesbegründung, 815 ,wird je nach Vertragstyp unterschiedlich ausfallen. Im Wesentlichen hängt die Beantwortung davon ab, ob und inwieweit die Krankenkassen auf die Auswahlentscheidung, welcher Vertragsgegenstand im einzelnen Versorgungsfall abgegeben wird, Einfluss nehmen.“

Da der Gesetzgeber im Zuge des KHRG ein Vordringen in den Selektivvertragsbereich für stationäre Leistungen nicht realisiert hat, ${ }^{816}$ werden u.a. Verträge zur IV in den Vordergrund rücken. ${ }^{817}$ Allerdings seien, so der Gesetzgeber,

„Verträge über eine integrierte Versorgung [nach] § 140a ff. in der Regel keine öffentlichen Aufträge, da die Entscheidung über den Abruf der jeweiligen Leistung nicht von den Krankenkassen, sondern von den Versicherten getroffen wird, die die angebotenen Versorgungsformen in Anspruch nehmen können. “818

Nicht ausgeschlossen ist damit jedenfalls, daß dies im Einzelfall auch anders sein kann. Diese Auffassung wurde auch von Generalanwalt Mazák in der Rs. Oymanns in Abgrenzung zur Dienstleistungskonzession betreffend einen IV-Vertrag über die Anfertigung und Lieferung von Schuhwerk bei diabetischem Fußsyndrom vertreten, und sodann vom EuGH bestätigt. ${ }^{819}$ Dabei ist einerseits zu Recht darauf hingewiesen worden, daß „,die Krankenkasse - und nicht, von geringen Zuzahlungen abgesehen, der Patient -

814 S. I.4.4.1. und I.4.4.5.

815 BT-Drs. 16/10609, S. 65.

816 Vgl. auch oben II.3.1.4.3.

817 S. auch II.2.1.2.

818 BT-Drs. 16/10609, S. 66.

819 Schlussanträge v. 16.12.2008, Rs. C-300/07, Rn. 63 ff.; EuGH, Urt. v. 11.6.2009, Rs. C-300/07 (Oymanns), NJW 2009, S. 2427 ff. 
die Vergütung schuldet“. 820 Überdies wird dem Einwand der „Unsicherheit das Zeitpunkts und des Umfangs der Inanspruchnahme“ entgegnet, ${ }^{821}$ die Erfüllungspflicht „auf Abruf“ entspreche der bei Rahmenvereinbarungen. ${ }^{822}$ Der Ausschreibungspflicht kann jedenfalls nicht die Nachrangigkeit spezifischer Gesundheitsleistungen entgegengehalten werden, da das nationale Recht unbeschadet der Anwendbarkeit der VOF nachrangige Leistungen nicht von der Pflicht zur Ausschreibung ausnimmt. ${ }^{823}$

Nach hier vertretener Auffassung kommt eine Ausschreibungspflicht für qualifizierte, insbesondere flächendeckende Integrations-Vollversorgungsverträge" in Betracht. ${ }^{824}$ Das gilt dann, wenn die IV unter Budgetverantwortung eine bevölkerungsbezogene Flächendeckung der Versorgung ermöglichen soll. Insoweit stehen meist nicht das „Ob“, sondern nur Zeitpunkt und Umfang der Inanspruchnahme nicht in jedem Einzelfall vorher fest. Das qualifizierte Modell der „Vollversorgung“ (umfassendes CareManagement) stellt sich weniger als völlig ungewisses Risiko der Inanspruchnahme, sondern eher als ein ,jedes Mal wenn“ dar.

Die Konsequenzen für den (Vertrags-)wettbewerb sind durchaus erheblich. Leistungserbringer werden durch eine Ausschreibung einerseits geschützt, sind andererseits allerdings daran gehindert, selbst innovative Konzepte zu offerieren, die durch exklusive Vertragspartnerschaft honoriert werden. Denn die Krankenkassen können sich angesichts solcher Offerten nur ,inspirieren“ lassen, eine Ausschreibung vorzunehmen, die entsprechende Inhalte publik macht, wodurch jedoch auch konkurrierende Leistungserbringer auf den Plan gerufen werden.

\subsection{Besonderheiten im Recht der PKV}

In der PKV stellt sich die (Rechts-)Lage mit Blickrichtung Vertragswettbewerb durchaus anders dar. Dies mag für den ausländischen Betrachter seltsam anmuten, mag man sich Managed Care-Modelle doch gerade im Bereich der Privatversicherung vorstellen. Allerdings fungieren Selektivverträge nach hiesigem Verständnis überwiegend (noch) als Grundlage besonderer Versorgungsformen und nicht - wiederum z.T. anders als im Ausland - als plurale Erscheinungsformen der Regelversorgung.

Das im PKV-Bereich weithin zu konstatierende Fehlen besonderer kollektiver und selektiver Verträge hat mehrere Gründe. Zum einen wird das Zulassungssystem innerhalb des GKV-Systems durch (fingierte) Versorgungsverträge allgemein mit Wirkung auch für die PKV organisiert; im übrigen gilt das Pflegesatzrecht hinsichtlich der allgemeinen Krankenhausleistungen ohnehin einheitlich für alle Benutzer, also auch privat

820 Schlussanträge, a.a.O., Rn. 69; s.auch EuGH, a.a.O., Rn. 73.

821 S. Görs, Krankenhäuser im Wettbewerb. Ausschreibung von Elektivleistungen, KHR 2008, S. 57 (59); Mazák, a.a.O., Rn. 70.

822 Görs, a.a.O., S. 59.

823 Vgl. I 4.4.5., Görs, a.a.O., S. 60; vgl. demgegenüber Engelmann (2009), juris-PK, § 69 Rn. 219 f.

824 Vgl. oben II.2.1.2. 
Versicherte. ${ }^{825}$ Vor allem aber kennzeichnet das Privatversicherungsrecht gerade kein Dreiecksverhältnis, da es auf den vertraglichen Beziehungen allein zum Versicherten fußt und dieser seinerseits mit dem Krankenhaus kontrahiert. Nach eigener Darstellung der PKV ${ }^{826}$ kommen hinzu der geringe Marktanteil sowie die Tatsache, daß die Patientenklientel ein hohes Interesse an der freien Arzt- und Krankenhauswahl hat. Managed Care hingegen würde - wie auch im Bereich der GVK gezeigt - gerade zur Einschränkung des Wahlrechts führen. 827

Nach dem Vorbild der IV bestünde überdies ein relativer „Regulierungsbedarf“ für selektive Verträge, der nicht nur Aspekte der ergänzenden Qualitätssicherung betrifft. Vielmehr müssten vor allem Möglichkeiten zum Dispens von der GOÄ geschaffen werden. Solche Möglichkeiten werden im Rahmen der freien Diskussion häufiger erwogen, sind aber - soweit ersichtlich - bisher nicht Gegenstand gesundheitspolitischer Aktivitäten.

All dies schließt nicht aus, daß auch die PKV-Versicherten mittelbar von den im Vertragswettbewerb erzielten Rationalisierungsgewinnen, die sich allgemein in den Organisations- und Behandlungsstrukturen der Krankenhäuser niederschlagen, profitieren. Ebenfalls nicht ausgeschlossen ist etwa ein Disease Management, das auf zusätzliche Beratungsangebote und (Informations-)Services setzt, ohne eine statusrelevante Einschreibung mit Einschränkung von Wahlrechten bzw. mit Auswirkungen auf die Gestaltung des Versicherungsverhältnisses zu verlangen. 828

Ein Bereich allerdings bleibt von besonderer Bedeutung: Über die Abrechnung der Wahlleistung „Unterkunft“ haben die Unternehmen der privaten Krankenversicherung mit mehr als $66 \%$ der Krankenhäuser Verträge abgeschlossen. ${ }^{829}$

\section{Wettbewerb zwischen Krankenhäusern}

Im Wettbewerbsverhältnis der Krankenhäuser untereinander gilt - v.a. für die Frage nach der Marktbeherrschung - als „,der sachlich relevante Markt ... der Angebotsmarkt für moderne Dienstleistungen, die in Krankenhäusern gegenüber Patienten erbracht werden“. $830 \mathrm{Da}$ für diesen vom BKartA fusionsrechtlich bestimmten Krankenhausmarkt „die ambulanten praxisärztlichen Behandlungen, Rehabilitationseinrichtungen sowie reine Privatkliniken nicht einzubeziehen sind“, hat für die Untersuchung allerdings nicht zur Folge, daß in diese Richtung bestehende Beziehungen außer Betracht

825 S. nachfolgend II.4.1.

826 Vgl. Gent, Integrierte Versorgung in der PKV (s. Literatur).

827 Siehe oben II.1.1.4.

828 Vgl. Benner, Disease-Management-Programme - Unterschiedliche Ansätze von privater und Gesetzlicher Krankenversicherung, Deutsches Ärzteblatt, Vol. 100, 2003, Heft 3, S. A82-A84.

829 S. DKI (Hrsg.), Krankenhaus Barometer, Umfrage 2005, S. 17.

830 BKartA, Beschl. v. 23.3.2005, B 10 - 109/04, Rn. 65. 
bleiben könnten. Im Gegenteil: Auch wenn es insofern nicht um (versicherte) „Krankenhausleistungen“"geht, markieren die letztgenannten Bereiche doch Wirkungsfelder, in denen die Krankenhäuser Einfluß zu nehmen suchen, z.B., um eine Einweisungssteuerung durch niedergelassene Ärzte, sektorenübergreifende Behandlungskonzepte oder freie Preisgestaltung durch eine Symbiose mit reinen Privatkliniken zu erreichen.

Der Wettbewerb zwischen den Krankenhäusern ist in erster Linie Wettbewerb um den Patienten und jedenfalls in seiner Bedeutung - äußerst grob skizziert - vergleichbar mit dem Mitgliederwettbewerb unter den gesetzlichen Krankenkassen. Der am Ende absatzorientierte Wettbewerb der Krankenhäuser um die Patienten vollzieht sich im Kontext der effizient vorgehaltenen Versorgungsstrukturen, ist also Wettbewerb um Fälle mit attraktiv oder als wirtschaftlich zu bezeichnender Vergütung.

Strukturkomponenten der Marktes für Krankenhausleistungen sind danach der $\mathrm{Zu}$ schnitt des Versorgungsangebotes, der eigentliche Absatzmarkt unter besonderer Berücksichtung möglicher Einweisungssteuerung sowie das Verhandlungsgewicht bei verbleibenden Möglichkeiten der Preisgestaltung (z.B. im Selektivvertragsbereich) bzw. der Beteiligung im Rahmen der Preisregulierung. Überdies lassen sich spezifische Erscheinungsformen des Wettbewerbs in einem Krankenhausmarkt im Umbruch ${ }^{831}$ konstatieren, so der Wettbewerb um Kooperationsgemeinschaften, die Neu- bzw. der Restrukturierung vieler Häuser i.V.m. einem Wettbewerb der Gesellschaftsformen und Organisationsmodelle, der Verdrängungswettbewerb und schließlich der Fusionswettbewerb.

\subsection{Markteintritt; Konkurrenz um die (Plan-)Position im regulierten System}

Wesentliche Vorwirkungen für Chancen und Verhalten am Markt vermittelt die Markteintrittsposition. Dabei geht es auch um den „Eintritt“ in das regulierte System und die Teilhabe an Privilegien. Gerade „unter Plankrankenhäusern herrscht Wettbewerb" auf einem „eng umschriebene[n] Markt »der Privilegierten«“. ${ }^{832}$ Erst die Aufnahme in den Krankenhausplan mit der Wirkung einer ,Mindestgarantie wirtschaftlicher Sicherheit“ ${ }^{\star 83}$ eröffnet diesen Markt. Infolgedessen herrscht Konkurrenz um die begrenzte - Planposition.

Der um die Planposition ringende Bewerber kann nur bedingt durch wettbewerbsorientiertes Verhalten Einfluss auf die Zuerkennung nehmen. Er muss v.a. seine für die Krankenhausanalyse der Krankenhausplanung erhebliche Leistungsfähigkeit nachweisen, sofern er seiner Angebotsstruktur nach überhaupt dem Bedarf (entsprechend der Bedarfsanalyse der Planung) entspricht. ${ }^{834}$ Erhält der die Aufnahme in den Plan begehrende Konkurrent einen entsprechend ablehnenden Bescheid, ist er, so die grundsätzli-

831 So auch der Titel des Krankenhaus-Reports 2006, hrsg. von Klauber/Robra/Schellschmidt (2007).

832 BVerwG, Urt. v. 25.9.2008, Az. 3C 35/07, GesR 2009, S. 27 (31).

833 Burgi, Konkurrentenschutz und Krankenhausplanung, NZS 2005, S. 169 (170); oben I.3.4.3.

834 Vgl. oben I.3.4.3. 
chen Erwägungen des $B V e r w G$ im September 2008, v.a. auf Rechtsschutz „,in eigener Sache“, d.h. gegen diesen Bescheid verwiesen. ${ }^{835} \mathrm{Da}$ Mitbewerbern gegenüber positive Feststellungsbescheide über die Aufnahme ergangen sind, stehe dem nicht entgegen. Streng genommen verneint das $B V e r w G$ damit die Drittwirkung des begünstigenden Bescheides. Es gesteht gleichwohl ein Rechtsschutzbedürfnis gegen eine mit Hilfsfunktion ausgestattete - flankierende - Anfechtungsklage gegen den Drittbescheid zu, wenn zwischenzeitlicher Vollzug der Drittbegünstigung droht und so die Erfolgsaussichten des Betroffenen, der selbst die Planaufnahme erstrebt, geschmälert sein könnten, v.a. dann, wenn der Dritte ebenfalls Neubewerber war und statt des Konkurrenten erstmals in den Plan aufgenommen wurde. ${ }^{836} \mathrm{Ob}$ dies konsistent ist und nicht vielmehr Drittwirkung grundsätzlich anzunehmen und zusätzlich vorläufiger Rechtsschutz in Betracht zu ziehen wären, 837 mag hier dahinstehen.

Umgekehrt kann 1t. BVerwG gegen eine Planherausnahme nur im Wege der dagegen gerichteten Anfechtungsklage vorgegangen werden. ${ }^{838}$ Indes wäre zu erwägen, ob konsequenterweise nicht auch in einer solchen Konstellation das „Interesse an der Unterbindung des Vollzugs der Aufnahmeentscheidung“ eine ähnliche Behandlung wie im Falle der „flankierenden Anfechtungsklage“ verlangte. ${ }^{839}$ Mindestens unzulässig, so ist der Rspr. zu entnehmen, sind solche Klagen von Konkurrenten mit Planposition, die im Grunde vorläufigen Rechtsschutz begehrend - die Aufnahme eines Konkurrenten in den Plan ohne eine damit (gleichzeitig) verbundene, d.h. nicht alsbald zum Vollzug anstehende Auswahlentscheidung der Behörde über Planherausnahmen angreifen; die Neuordnung ist dann abzuwarten. 840

Abgesehen davon betrifft die Planaufnahme eines Bewerbers als solche ein vorhandenes Plankrankenhaus nicht in dessen Rechten. ${ }^{841}$ Eine eigenständige Klagebefugnis lässt sich insbesondere ${ }^{842}$ weder aus der Befürchtung ableiten, im Zuge der Konkurrenz könnten für die Erbringung (zu Lasten der GKV) erforderliche Mindestmengen (§ 137 Abs. 3 S. 1 Nr. 2, S. 2 SGB V) nicht mehr erreicht werden, noch besteht ein subjektives Recht eines Plankrankenhauses auf Vermeidung von Überversorgung.

835 BVerwG, Urt. v. 25.9.2008, Az. 3C 35/07, GesR 2009, S. 27 (29).

836 A.a.O., S. 30.

837 Bracher, Anm. zu BVerwG, Urt. v. 25.9.2008, Az. 3C 35/07, DVB1. 2009, S. 49 (50).

838 BVerwG, Urt. v. 25.9.2008, Az. 3C 35/07, GesR 2009, S. 27 (30).

839 Bracher, Anm. zu BVerwG (a.a.O.), DVBl. 2009, S. 49 (50).

840 BVerwG, Urt. v. 25.9.2008, Az. 3C 35/07, GesR 2009, S. 27 (31).

841 A.a.O.

842 Umfassend BVerwG, a.a.O., S. 31 ff. 


\subsection{Angebotswettbewerb und Preiswettbewerb}

In Anbetracht des Systems der Fallpauschalen als Festpreisen ist bereits darauf hingewiesen worden, daß die Verhaltensspielräume der Krankenhäuser in aller Regel nicht die Preisgestaltung betreffen; ${ }^{843}$ die Monopolkommission konstatiert:

„Daher existiert gegenwärtig kein spürbarer Preiswettbewerb auf den deutschen Krankenhausmarkt." 844

Maßgeblich sind vielmehr das Versorgungsangebot und die Spezialisierung des Hauses sowie die Qualität der Krankenhausleistung.

Dies gilt umso mehr, als sich in der sog. Konvergenzphase die Spannweite der Basisfallwerte der Krankenhäuser auf dem Weg der Annäherung an den landeseinheitlichen Basisfallwert von 2005 bis 2007 mehr als halbiert hat. ${ }^{845}$ Insofern gab es - auf dem Weg zu einheitlichen Wettbewerbsbedingungen, wenngleich ohne wesentlichen Preiswettbewerb - „Konvergenzgewinner“ und „-verlierer“. 846 Die vollständige Annäherung wird diese Unterschiede ebenso nivellieren wie sie beinahe zwangsläufig zu effizienzorientierten Restrukturierungsbemühungen geführt hat. Gleichzeitig wird mittelfristig die Entwicklung hin zu einem auch bundeseinheitlichen Basisfallwert erwartet. ${ }^{847}$

\subsubsection{Preisgestaltung nach Art der Leistung}

Inwieweit den Krankenhäusern überhaupt Preisgestaltungsspielräume zustehen, ist abhängig von der Art der Leistung. $\mathrm{Zu}$ unterscheiden sind insofern allgemeine Krankenhausleistungen, wahlärztliche und Wahlleistungen der Unterkunft, sonstige medizinische Wahlleistungen sowie solche im Rahmen der IV. Die für ambulante Leistungen nach $\S 116 \mathrm{~b}$ SGB V vor der Reform durch das GKV-WSG noch bestehenden marginalen Verhandlungsspielräume für die Vergütung der vormals einzelvertraglich zu vereinbarenden ambulanten Behandlung im Krankenhaus sind nach der Reform entfallen bzw. in Form von Maßstäben an die Abrechnung der vertragsärztlichen Leistungen angepasst worden.

Entgelte für allgemeine Krankenhausleistungen müssen für alle Benutzer des Krankenhauses einheitlich berechnet werden; die Fallpauschalen gelten damit auch für die Versorgung privat Versicherter. ${ }^{848}$ Dasselbe gilt für Pflegesätze und Vergütungen der vor- und nachstationären Behandlung. ${ }^{849}$ Begrifflich Pflegesätze, ${ }^{850}$ sind auch Wahl-

843 II.1., II.1.1.3.

844 Siebzehntes Hauptgutachten, BT-Drs. 16/10140, S. 319, Ziff. 817.

845 Leclerque/Friedrich, in: Klauber/Robra/Schellschmidt (Hrsg.), Krankenhaus-Report 2008/2009 (2009), S. 229 (240).

846 A.a.O., S. 231.

847 A.a.O., S. 240.

$848 \S \S 8$ Abs. 1 S. 1 KHEntgG, $\S 17$ Abs. 1 S. 1 KHG, $\S 14$ Abs. 1 HS. 1 BPflV.

849 Vgl. § 17 KHG; ausführlich unter I.3.4.2. 
leistungen (zwischen Krankenhäusern und Patienten) für alle Benutzer einheitlich abzurechnen, wobei die GOÄ weithin entsprechende Anwendung findet. ${ }^{851}$ Gemäß entsprechender Tarife übernehmen die privaten Versicherungen die Kosten für die wahlärztliche (v.a. Chefarztbehandlung) sowie die Wahlleistung Unterkunft (als nichtwahlärztliche Leistung).

Bei alledem bedeutet die pauschale Abgeltung über das DRG-System nicht, daß ein solches nicht grundsätzlich auch für eine preiswettbewerbliche Komponente tauglich sein könnte. Das wäre aber nur dann der Fall, wenn das Bewertungsmodul im Sinne wettbewerblich ausgehandelter Basisfallwerte pro Haus ausgestaltet würde. ${ }^{852} \mathrm{Im}$ deutschen System landeseinheitlicher, kollektiv ausgehandelter Basisfallwerte nach $\S 10$ KHEntgG ist dies allerdings gerade nicht vorgesehen.

Auch hinsichtlich der beschriebenen Wahlleistungen bleiben der Gestaltungsspielraum und die damit verbundene Möglichkeit der Steigerung des wirtschaftlichen Erfolges gering. Daher wird mitunter versucht, das gesamte Leistungsspektrum (allgemeine Krankenhausleistungen und Wahlleistungen) außerhalb der öffentlich-rechtlichen Vergütungsbestimmungen abzurechnen. ${ }^{853}$ Da dies - die Zulässigkeit zunächst unterstellt organisatorische Strukturen im Sinne einer Symbiose von gefördertem Krankenhaus und ,privatklinischen Einrichtungen“ voraussetzt, soll diese Frage im Zusammenhang mit der wettbewerbsorientierten strukturellen Reorganisation vieler Krankenhäuser beleuchtet werden. 854

Unbeschadet dessen können „sonstige Wahlleistungen“ in Form medizinischer Wahlleistungen erbracht werden, die mangels Anknüpfung an die Arztwahl nicht als wahlärztliche Leistungen zu bezeichnen sind. ${ }^{855}$ Sie sind Fallgruppen v.a. der fehlenden medizinischen Indikation (wie z.B. bei der ästhetischen Chirurgie) sowie Alternativleistungen zuzuordnen, die sich durch einen höchst innovativen Produkt- und Qualitätsstandard auszeichnen, hinsichtlich dessen kein Kostenübernahmeanspruch besteht. ${ }^{856}$ Deren dennoch ausbedungene Erstattung setzt zahlungskräftige Patienten voraus, zumal die Tarifbedingungen der PKV-Unternehmen mit Blick auf die „medizinisch notwendige Heilbehandlung" hier meist nicht erfüllt sein werden. Der Ausrichtung an

850 Quaas, in: Wenzel (Hrsg.), Handbuch des Fachanwalts Medizinrecht (2007), Kap. 12, S. 1137 Rn. 83.

851 Quaas, a.a.O.

852 Siehe Neubauer, Zur ökonomischen Steuerung der Krankenhausversorgung unter DRGFallpauschalen, in: Klauber/Robra/Schellschmidt, Krankenhaus-Report 2003 (2004), S. 101 (109 f.).

853 Quaas, Sind Wahlleistungen und Privatkliniken eine finanzierungsrechtliche Alternative?, KHuR 2006, S. 114 (119 ff.).

854 Siehe II.4.7.2.

855 Quaas, Sind Wahlleistungen und Privatkliniken eine finanzierungsrechtliche Alternative?, KHuR 2006, S. $114 \mathrm{ff}$.

856 Quaas, a.a.O., S. $118 \mathrm{ff}$. 
medizinischen Wahlleistungen ist daher bescheidener Erfolg zu attestieren: „Das Krankenhaus wird daran gewiss nicht finanziell genesen“. 857

Anders stellt sich die Lage dar, wenn spezialisierte Privatkliniken überhaupt nur solche Leistungen anbieten oder als reine Privatkliniken ohne Planstatus und organisatorische Verbindung zu solchen Plankrankenhäusern rein privatrechtlich abrechnen. Dann gelten die öffentlich-rechtlichen Vergütungsbestimmungen nicht. Da die Kliniken hier allein auf den Patienten als Selbstzahler angewiesen sind und im übrigen in kleiner Zahl bestehen (2004: 130), 858 sind sie nach Auffassung des BKartA nicht dem allgemeinen Krankenhausmarkt zuzurechnen. 859

Damit bleibt für die weitergehende selektivvertragliche Preisgestaltung im Krankenhausbereich wenig Raum. Die vormals existente Vertragskompetenz im Bereich des $\S 116 \mathrm{~b}$ SGB V ist - ungeachtet der ohnehin gem. S. 2 (a.F.) vermittelten Einschränkungen hinsichtlich der Vergütung - entfallen. Darüber hinaus und unbeschadet der vormals gebundenen Anschubfinanzierung (vgl. § 140d Abs. 4 SGB V) bleibt nur die individuelle Preisgestaltung im Rahmen der integrierten Versorgung möglich. ${ }^{860}$

Festzuhalten ist danach, daß vorbehaltlich des Vordringens der Krankenhäuser in den ambulanten und den Schnittstellenbereich die Möglichkeiten zur Preisgestaltung und zur Schaffung dadurch bedingter Wettbewerbsvorteile gering sind.

\subsubsection{Qualität, Versorgungsangebot und Spezialisierung}

a) Die zuletzt genannten Versorgungsformen markieren jedoch ein besonderes Feld mit der Möglichkeit zur Spezialisierung und Steigerung der Qualität, die die Akquisition der Patienten maßgeblich bestimmt, wenngleich angesichts einer Informationsasymmetrie nur in eingeschränktem Umfang. ${ }^{861}$ Die Veröffentlichung der Qualitätsberichte im Internet i.V.m. mit der Handlungsalternative vergleichenden Informierens und des Aussprechens von Empfehlungen durch Krankenkassen und Kassenärztliche Vereinigungen (§ 137 Abs. 3 S. 1 Nr. 4, S. 4 SGB V) macht das Qualitätskriterium jedenfalls operabel.

Der im Hinblick auf die Wahl des Krankenhauses bereits näher betrachtete Wettbewerbsparameter "Qualität“ ist von allgemeiner Bedeutung auch dann, wenn er von den Nachfragern nur unvollkommen wahrgenommen wird. Das ergibt sich bereits aus den gesetzlichen Vorgaben der Qualitätssicherung (vgl. v.a. § 137 SGB V), die das Leistungsniveau in puncto Versorgungsqualität nach $\S 137 \mathrm{a}$ SGB V mess- und darstellbar ${ }^{862}$ bestimmen. In diesem Zusammenhang ist die (nunmehr) mit Richtliniencharak-

857 Quaas, a.a.O., S. 119.

858 Vgl. BKartA, Beschl. v. 23. März 2005, B 10 - 109/04, Rn. 73 Fn. 93.

859 Vgl. BKartA, Beschl. v. 23. März 2005, B $10-109 / 04$, Rn. 73.

860 S.o. II.3.1.4.3.

861 II.1.1.3.

862 Durch ein unabhängiges Qualitätsinstitut; als solches fungierte zunächst die Bundesgeschäftsstelle Qualitätssicherung (BQS), vgl. insoweit zum Qualitätsreport 2007 auch Veit, Qualitätssicherung im 
ter ausgestattete Vereinbarung zur Qualitätssicherung für Krankenhäuser des Gemeinsamen Bundesausschusses zu nennen; 863 im Wege der Anlage einbezogen sind verpflichtend zu dokumentierende Leistungsbereiche. Ebenfalls der Qualitätssicherung zuzurechnen sind die zur Teilnahme am Markt wichtigen Mindestmengen, die sich aus der mit Richtlinienqualität (vgl. § 137 Abs. 1 S. 1 i.V.m. § 92 Abs. 1 S. 2 Nr. 13 SGB $\mathrm{V})$ ausgestatteten Mindestmengenvereinbarung ergeben. ${ }^{864}$ Die zuletzt in der Anlage 1 (OPS Version 2009) ausgewiesene jährliche Mindestmenge pro Krankenhaus etwa beträgt bei Lebertransplantationen 20 und bei Nierentransplantationen 25 .

Dessen ungeachtet wird es stets regulatorischer Vorgaben bedürfen, um Qualität im sensiblen Gesundheitssektor als einem unvollkommenen Markt sicherzustellen. Über beides geht die Monopolkommission hinweg, wenn sie konstatiert:

„Die insgesamt geringe Qualitätstransparenz im Krankenhaussektor diszipliniert Krankenhäuser derzeit nur unzureichend in ihren Anreizen, die Behandlungsqualität abzusenken." “865

Gleichzeitig weist sie darauf hin, die Steigerung des Wettbewerbs führe in „Bereichen, die sich Qualitätstransparenz entziehen, zu einer Qualitätsreduktion“.866 Dennoch gelte:

„Gesetzliche Qualitätsvorgaben für Krankenhausleistungen sind ungeeignet, ein hohes Qualitätsniveau zu garantieren." 867

Eine andere Frage ist es hingegen, wenn pauschalierte Entgelte eine verfrühte, „blutige“ 868 Entlassung zur Folge haben und an anderer Stelle im System (ambulante) Folgekosten generieren. 869

b) Der Angebotsmarkt wird im wesentlichen von den Allgemeinkrankenhäusern und den Fachkliniken bedient. In Anbetracht der Existenz von Krankenhäusern der Grund-, Regel-, Schwerpunkt- und Maximalversorgung decken Krankenhäuser der höheren Versorgungsstufe 870 zumindest auch die Bereiche der niedrigeren Versorgungsstufen ab. ${ }^{871}$ In diesem Sinne konkurrieren etwa Allgemeinkrankenhäuser ebenso mit den be-

Krankenhaus, ErsK 2008, S. 423 ff.; zuletzt wurde das „Aqua-Institut für angewandte Qualitätsför-

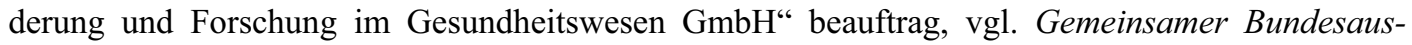
schuss (Hrsg.), Newsletter 2/2009.

863 Zul. geänd. am 19.2.2009, BAnz. 2009, S. 1271.

864 Zul. geänd. am 18.12.2008, BAnz. 2009, S. 4809.

865 Siebzehntes Hauptgutachten, BT-Drs. 16/10140, S. 321, Ziff. 822.

866 A.a.O., S. 321, Ziff. 821.

867 A.a.O., S. 320, Ziff. 819.

868 A.a.O., S. 324, Ziff. 831.

869 A.a.O., S. 321, Ziff. 822.

$870 \mathrm{Zu}$ den Versorgungsstufen I-III, neben den Fachkrankenhäusern des Krankenhausplanes des Freistaates Bayern 2007, vgl. dessen Teil I, Allgemeine Grundsätze, Ziff. 5.2.1.-5.2.3.

871 Vgl. BKartA, Beschl. v. 23. März 2005, B $10-109 / 04$, Rn. 78. 
nachbarten wie mit Fachkliniken. ${ }^{872}$ Der Patient hingegen nimmt das Angebot einheitlich wahr, auch wenn er im Bedarfsfalle stets Teilleistungen nachfragt. ${ }^{873}$

In diesem Zusammenhang spielt die Bindung des Patienten an bestimmte Häuser eine nicht zu unterschätzende Rolle, wobei tendenziell Krankenhäuser im Vorteil sind, die ein umfassendes Leistungssortiment anbieten. Qualitätsberichte vermögen eine entsprechende Bindung mitunter zu durchbrechen. Wiederum andere Patienten fühlen sich eher sortimentsunabhängig dem - z.B. kirchlichen - Träger der Krankenhäuser verbunden.

Sowohl Ausdruck des Angebotszuschnitts als auch der Kooperation mit anderen Krankenhäusern ist die qualitätssichernd erfolgende Abstimmung der Leistungsspektren der Krankenhäuser untereinander. Das Krankenhaus Barometer (Umfrage 2007) weist hinsichtlich der stationären Leistungen Abstimmungsaktivitäten von einem Viertel der Häuser aus; im ambulanten Bereich sind es ca. 10\%. ${ }^{874}$

\subsubsection{Absatzmenge}

Im Rahmen betriebswirtschaftlicher Überlegungen spielt naturgemäß die Absatzmenge eine Rolle. Die gewinnorientierte Erfolgswirksamkeit ergibt sich in Abhängigkeit der Relevanz der Behandlungsleistungen bzw. Fälle entsprechend der Leistungsstrukturen im Versorgungsmanagement und nach Maßgabe einer positiv aufwandsorientierten Darstellung im case-mix.

Gleichzeitig impliziert der Wettbewerb um die Leistungsmenge einen solchen um Strukturvorteile in der Regulierung bzw. um den Erhalt der systemimmanent gefestigten Marktposition. Insoweit stellen sich die Regelungen über Mindestmengen (vgl. § 137 Abs. 3 S. 1 Nr. 3, S. 2 SGB V) nicht allein als Qualitätssicherungsmaßnahmen, sondern aus Sicht der Krankenhäuser als Marktteilnehmer auch als „Zweite Komponente wirtschaftlicher Mindestsicherung “875 dar. Umgekehrt könnte man auch von einer zweiten Hürde im Zeichen besonderer Regulierungsverdichtung sprechen. Dabei kann es zu Zielkonflikten kommen, bezieht man die Krankenhausplanung im Spannungsfeld zwischen Bedarfsanalyse und grundrechtlichen Marktein- und Zutrittschancen in die Betrachtung mit ein. Qualitäts- und Versorgungsziele können mithin miteinander konfligieren. 876

\subsubsection{Exkurs: Einflüsse veränderten Kodierverhaltens}

Die im Bereich der DRG bekannte Problematik der Einflussnahme auf die erlösrelevante Behandlungsdokumentation in Form des sog. ,up-codings“ oder „gamings“ 877 ist

872 A.a.O.

873 A.a.O., Rn. 79.

874 DKI (Hrsg.), Krankenhaus Barometer, Umfrage 2007, S. 26 f.

875 Vgl. oben II.4.1.

876 Becker, in: ders./Kingreen, SGB V (2008), § 137 Rn. 12.

877 S. Wasem/Rotter/Focke/Igl, in. Klauber/Robra/Schellschmidt, Krankenhaus-Report 2007 (2008), S. $49 \mathrm{ff}$. 
letztlich ein allgemeines Problem, das sich im Gefolge der „Klassifikation“ von Fällen der Krankenhausbehandlung ebenso zu stellen vermag, wie bei einer solchen von Versicherten nach Morbiditätsgruppen. Während die Problematik des sog. ,up-“ bzw. „rightcodings" im Hinblick auf den M-RSA v.a. ambulante Diagnosen betrifft und die Finanzbasis der Krankenkassen tangiert, ${ }^{878}$ ist das seitens der Krankenhäuser „,veränderte Kodierverhalten als eine der ungeklärten Herausforderungen der DRG-Einführung“"879 bereits länger Gegenstand der Diskussion. Die Versuchung lässt sich kaum nicht negieren:

„Das Interesse eines Krankenhauses an einer möglichst hohen Wertigkeit der behandelten Fälle
mag über das »Rightcoding« hinaus aber auch Überlegungen auslösen, durch Manipulationen der
Dokumentationen gegenüber dem realen Leistungsgeschehen hinsichtlich der Höhe der abrechen-
baren Bewertungsrelationen »nachzuhelfen«, wofür sich der Begriff des »Upcoding« eingebürgert
hat.“ 880

Auch die Monopolkommission hat „manipulatives Kodierverhalten“ kritisiert und u.a. auf die regelmäßige Kodierung von Geburtskomplikationen hingewiesen. 881

Wichtig ist, daß es aus normativer Warte allein auf die Einhaltung der Kodier- und Meldevorschriften ankommt, wohingegen der verklärende Begriff des sog. „rightcodings“ häufig „operationalisiert" wird, um sachverhaltsunspezifisch Bestrebungen ohne Betrachtung der Kodier-, berufs-, datenschutz- und abrechnungsrechtlicher Vorschriften zu legitimieren. Insbesondere die manipulatorisch „korrigierende Nacherfassung“" von Diagnosen rührt an zentralen Steuerungskomponenten des Systems. Legalistisch gesehen kann hier auch nicht von Fehlanreizen gesprochen werden, unbeschadet der Frage erforderliche Kontrolle.

Soweit im übrigen nur die „nominelle Ebene“ 882 betroffen ist, sollen „Right-“ wie „Upcoding“ nach dem gesetzgeberischen Willen jedenfalls nicht zu Mehrerlösen führen, soll die effiziente Allokation also nicht konterkariert werden. Auch im Bereich der Anpassung der vertragsärztlichen Gesamtvergütung an die Morbiditätsentwicklung im Zuge des GKV-WSG hat der Gesetzgeber deutlich gemacht, daß „Mehrleistungen, die... durch statistische Effekte wie z.B. das „Up-“ oder auch „Right-Coding“ anfallen,... nicht zusätzlich von den Krankenkassen vergütet“" werden. ${ }^{883}$. Auf die Details zur Regulierung und „Isolierung“ der Ergebnisse veränderten Kodierverhaltens kann hier nicht näher eingegangen werden. ${ }^{884}$

878 Dazu unter II.5.1.2.

879 Gleichnamiger Titel des Beitrags von Wasem/Rotter/Focke/Igl, in: Klauber/Robra/Schellschmidt, Krankenhaus-Report 2007 (2008), S. 49 ff.

880 A.a.O., S. 49 ff.

881 Monopolkommission, Siebzehntes Hauptgutachten, BT-Drs. 16/10140, S. 325 f., Ziff. 837.

882 Wasem/Rotter/Focke/Igl, in: Klauber/Robra/Schellschmidt, Krankenhaus-Report 2007 (2008), S. 50.

883 BT-Drs. 16/3100, S. 120.

884 Dazu wiederum Wasem/Rotter/Focke/Igl, in. Klauber/Robra/Schellschmidt, Krankenhaus-Report 2007 (2008), S. 49 ff. 


\subsubsection{Besondere Versorgungsformen: Konzeption und Haftung}

Besondere Versorgungsformen wie DMP und insbesondere (deren Durchführung im Wege der) IV spielen nicht nur im Verhältnis der Krankenkassen zu den teilnehmenden Versicherten ein Rolle, sondern auch mit Blick auf die Binnenkonkurrenz der Leistungserbringer um den Zusammenschluss in Netzwerken ${ }^{885}$ und um den Abschluß von Selektivverträgen mit den Krankenkassen. ${ }^{886}$ Dies zusammengenommen erscheinen besondere Versorgungsformen zunehmend als notwendiger Bestandteil der „Angebotspalette" der Krankenhäuser im Wettbewerb untereinander und zur mindestens indirekten Steuerung der Akquisition von Patienten. Die hier verbleibenden Spielräume bei der Preisgestaltung untermauern dies. ${ }^{887}$

Konkurrenzfähigkeit in diesem Bereich bedeutet aus Sicht der Krankenhäuser dabei großen Planungs- und Organisationsaufwand sowohl im medizinischen als auch im ökonomischen, organisatorischen und juristischen Bereich. Für die Kalkulation ist angesichts des massiven Projektaufwandes eine exakte Analyse der wirklichen Aufwendungen auf der Grundlage der eigenen Zahlen erforderlich. „Sonst kann es passieren, daß $10 \%$ des Gesamtarbeitsaufwandes darauf verwendet werden, 1\% Erlössteigerung zu bewirken." 888 Das galt zunächst umso mehr, als mit der nach $§ 140$ c Abs. 1 S. 1 SGB V mit den Krankenhäusern zu finanzierenden Vergütung bis zum 31. Dezember 2008

„nur die Leistungen finanziert [wurden], die über die im Gesamtbetrag nach den $\S \S 3$ und 4 des

Krankenhausentgeltgesetztes oder dem $\S 6$ der Bundespflegesatzverordnung enthaltenen Leistungen hinaus vereinbart werden."

Danach mussten besondere Rationalisierungsgewinne erwirtschaften werden, um in Anbetracht der Rechnungskürzung nach $\S 140 d$ Abs. 1 SGB V das vor Einführung der Anschubfinanzierung erzielte Vergütungsniveau zu halten. ${ }^{889}$

„Die Anschubfinanzierung... konkurriert deshalb mit dem gesetzlichen Auftrag nach $\S 1$ Abs. 1 KHG, der wirtschaftlichen Sicherung der Krankenhäuser“".890

Unbeschadet der Anschubfinanzierung aber kann gleichwohl von den Vorschriften des KHG und KHEntgG abgewichen werden, wenn dies der Förderung qualitativ hochwertiger Versorgung im Integrationsverbund dient.

Überdies entbinden die in IV-Modellen zumeist definierten medizinischen Behandlungspfade nicht von den (arzt-) haftungsrechtlichen Anforderungen und sind daher mit

885 Vgl. nachfolgend II.4.3.

886 Dazu II.3.1.2.

887 Vgl. vorstehend II.4.1.

888 Mau, Integrierte Versorgung - Chancen und Risiken aus der Sicht der klinischen Praxis, KHuR 2004, S. 71 (75).

889 Bohle, Integrierte Versorgung - Aktuelle Rechtsfragen der Umsetzung, in: ders. (Hrsg.), Vertragsgestaltung in der Integrierten Versorgung (2005), S. 9 (17).

890 Bohle, a.a.O., S. 18. 
Risiken verbunden, ${ }^{891}$ wenn die Kongruenz der Standards nicht hinreichend bedacht wird. Das Problem ist ein doppeltes: In besonders gelagerten Fällen könnte ein Festhalten am Behandlungspfad unter Umständen eine Verletzung der erforderlichen Sorgfalt und damit des Behandlungsvertrages mit dem Patienten bedeuten, während umgekehrt ein Abweichen zur Verletzung des öffentlich-rechtlichen Versorgungsvertrags führt. ${ }^{892}$ Dem zu begegnen, bieten sich der Flexibilität Rechnung tragende Vertragsklauseln bzw. Kontrollen durch Fachausschüsse an. 893

\subsection{Einweisungssteuerung seitens der Krankenhäuser}

Wie gesehen wird der Absatzmarkt weniger über den Preis als vielmehr durch die Qualität und das Versorgungsangebot bestimmt. Diese Parameter setzen bei der Wahl des Krankenhauses durch die Patienten an. ${ }^{894}$ Unbeschadet der „neben“ ärztlicher Einweisungstätigkeit ausgeübten Wahlrechte aber ist die Nachfragetätigkeit und -macht der Patienten begrenzt, weniger ,aufgrund ihrer Zahl“ 895 als vielmehr durch die Begrenzung auf indizierten Behandlungsbedarf, deretwegen auch die Kostenträger „keinen relevanten Einfluß auf das mengenmäßige Volumen der Nachfrage“" haben. ${ }^{896}$

Angesichts dessen stellt sich für das Krankenhaus die Frage, wie es individuell oder in Kooperation mit anderen Häusern auf die Steuerung der Patientenströme Einfluß nehmen kann. Insofern liegt nahe, auch bei den Ärzten anzusetzen. Während allerdings die Gründung von und Steuerung über MVZ in der Trägerschaft der Krankenhausträger grundsätzlich zulässig ist, begegnen Einweisungspauschalen als „Entgelte“ an Ärzte bereits im Ansatz Bedenken, wobei hier die konkrete Ausgestaltung entscheidend ist. Unter Wettbewerbsgesichtspunkten ebenfalls kritisch zu beurteilen sind Vereinbarungen zwischen Krankenhäusern über die Beteiligung an Versorgungskonzepten und die Steuerung von Patientenströmen.

\subsubsection{Gründung von und Steuerung durch MVZ}

Ein strategisches Einweisungsmanagement können die Krankenhäuser bspw. im Gefolge der Gründung von MVZ durch ihre eigenen Träger avisieren. MVZ werden meist in (z.T. unmittelbarer) Nähe des Krankenhauses errichtet. Letztere sind schon von daher „nächsterreichbar“ i.S.d. § 73 Abs. 4 SGB V. Zu dieser organisatorischen kommt nach Inkrafttreten des $V$ ÄndG auch eine mögliche personelle Verschränkung. Entsprechend der Änderung der auch für MVZ maßgeblichen Zulassungsverordnung für Vertragsärzte

891 Siehe Koller: Haftungsrechtliche Probleme im Rahmen der Integrierten Versorgung nach dem GMG, KHuR 2005, S. 71 ff.

892 Koller, a.a.O., S. 72.

893 Koller, a.a.O., S. 72 f.

894 Vgl. II.1.1.1.

895 So BKartA, Beschl. v. 23.3.2005, B 10 - 109/04, Rn. 135.

896 A.a.O. 
(Ärzte-ZV), § 1 Abs. 3 Nr. 2, ist die Tätigkeit in oder die Zusammenarbeit mit einem zugelassenen Krankenhaus nach $\S 108$ SGB V mit der Tätigkeit des Vertragsarztes vereinbar, § 20 Abs. 2 S. 2 Ärzte-ZV.

Die Gründung von MVZ erlangt mithin nicht allein als Strategie im Verhältnis der Konkurrenzsituation der Krankenhäuser untereinander Bedeutung. Vielmehr haben - in personell-organisatorischer Hinsicht - auch die im MVZ angestellten Krankenhausärzte teil an der allgemeinen Konkurrenzsituation zwischen MVZ und niedergelassenen Ärzten. ${ }^{897}$ Leistungsbezogen hingegen konkurrieren (v.a. nicht in Krankenhausträgerschaft befindliche) MVZ allein mit solchen Krankenhäusern, die ausnahmsweise vertragsärztliche Leistungen erbringen. 898

Zentrale Bedeutung im Verhältnis der Konkurrenz der Krankenhäuser untereinander allerdings entfaltet die Einweisungssteuerung über die von den Trägern der stationären Einrichtungen gehaltenen MVZ. In beiden Untersagungsverfügungen des BKartA gegenüber der Rhön-Klinikum AG, andere Häuser zu übernehmen, wurde die „Einführung“ 899 bzw. „Übernahme“ 900 von MVZ als Möglichkeit zur „Akquisition stationärer Patienten durch ambulante Versorgung “901 im Sinne der Einflußnahmemöglichkeit auf potentielle Patientenströme gesehen. Insofern entstünden Wettbewerbsvorteile und die Möglichkeit der Erzielung von Synergieeffekten für das Kerngeschäft in erheblicher Weise. 902

Von einem „Siegeszug“ dieser Steuerungsoption durch Gründung von MVZ in der Hand der Krankenhäuser lässt sich allerdings derzeit nicht sprechen. Vielmehr hat sich in der Praxis erwiesen, daß zahlreiche Häuser ihre zunächst intensiv betriebenen Bemühungen (vorerst) eingestellt haben. ${ }^{903}$ Damit hat sich die im Jahr 2005 geäußerte Prognose, bis zum Jahre 2007 würden 500 krankenhausbetriebene MVZ errichtet, ${ }^{904}$ nicht bewahrheitet. Sie beruhte auf der Einschätzung, daß 28\% der Krankenhäuser innerhalb der auf das Jahr 2005 folgenden 2 Jahre die Einrichtung eines MVZ planten. ${ }^{905}$ Nach Erhebungen der Kassenärztlichen Bundesvereinigung (KVB) waren zum 3. Quartal 2008 insgesamt 1.152 MVZ zugelassen, in denen 5.183 (durchschnittlich 4,5) Ärzte tätig waren, 3.921 (76\%) davon im Anstellungsverhältnis. ${ }^{906}$ 37,2\%, das sind 429 und

897 Vgl. Kuhlmann, das Krankenhaus 2004, S. 13.

898 Kuhlmann, a.a.O.

899 BKartA, Beschl. v. 23.3.2005, B $10-109 / 04$, Rn. 130.

900 BKartA, Beschl. v. 10.3.2005, B 10 - 123/04, Rn. 172.

901 BKartA, Beschl. v. 23.3.2005, B 10 - 109/04, Ziff. 3.3.2 vor Rn. 129; Beschl. v. 10.3.2005, B 10 123/04, Ziff. 3.4.2. vor Rn. 171.

902 BKartA, Beschl. v. 10.3.2005, B 10 - 123/04, Rn. 173 f.; Beschl. v. 23.3.2005, B 10 - 109/04, Rn. $131 \mathrm{f}$.

903 Dazu und den Gründen DKI (Hrsg.), Krankenhaus Barometer, Umfrage 2006, S. 51.

904 Andreas, ArztRecht 2005, S. 144.

905 Andreas, a.a.O. m.N.

906 Siehe unter www.kbv.de (Fachbesucher, Themen, MVZ). Allein 243 MVZ, davon 58 in Krankenhausträgerschaft, existierten in Bayern, gefolgt von Berlin (119/28) und Niedersachsen (113/48); Stand: 3. Quartal 2008. 
damit immer noch deutlich unter 500 Einrichtungen, befinden sich in der Trägerschaft von Krankenhäusern. 907

Von den Krankenhäusern, die ihre Bemühungen um die Gründung eines MVZ (vorerst) eingestellt hatten - bezogen auf $341 \mathrm{im} \mathrm{Jahr} 2006$ befragte Häusern - gaben 57,1\% starke Widerstände der niedergelassenen Ärzte, 41,4\% Zulassungssperren für bestimmte Fachgebiete und $20,7 \%$ juristische Probleme ${ }^{908}$ als wesentliche Gründe für den Rückzug an.

Auch in Anbetracht des VÄndG hat sich die Quote der MVZ in Krankenhausträgerschaft 909 nicht signifikant verändert. Sie lag längere Zeit knapp unter 33\% und liegt seit dem 4. Quartal 2007 trotz des Rückgangs der Zahl der Krankenhäuser bei etwas über einem Drittel. 910 Die Öffnung für die Tätigkeit von Vertragsärzten hat den Anstieg nicht beflügelt, denn in MVZ in Trägerschaft eines Krankenhauses arbeiten überwiegend angestellte Ärzte, so im 3. Quartal 2008 insgesamt 1.901 gegenüber 44 Vertragsärzten. 403 Einrichtungen arbeiten ausschließlich mit angestellten Ärzten.

Die krankenhausbezogen besondere Bedeutung der MVZ für das Einweisungs- und Entlassungsmanagement rückt diese - nebst anderen Strategien der „Zentrenbildung“911 - in den Blickpunkt einer Versorgungszentrierung als Teil der strukturellen Reorganisation. 912

\subsection{2. „Einweisungspauschalen“ an Ärzte}

\subsubsection{Die Problematik}

Eine andere Möglichkeit der Einweisungssteuerung setzt direkt bei den niedergelassenen Ärzten an, denen Pauschalentgelte seitens der Krankenhäuser für bestimmte im Zusammenhang mit der Krankenhausbehandlung stehende Tätigkeiten gezahlt werden. Die sozialrechtlich intendierte Kooperation von ambulantem und stationärem Sektor fordert hier in besonderer Weise das Berufsrecht heraus, denn Zuweisungsentgelte sind nach $\S 31 \mathrm{MBO}$ in Gestalt der Landesberufsordnungen verboten. 913 Mangels unausweichlicher Normenkollision kann dabei das Berufsrecht nicht in toto mit dem Hinweis auf die Normenhierarchie ,ausgehebelt“ werden. Dies widerspräche methodischen Grundsätzen, führte zur allgemeinen Derogation weiter Anwendungsbereiche des Berufsrechts und liefe im Kernbereich schließlich der Rechtssetzungsautonomie vor dem

907 A.a.O.

908 „Aufgrund der komplizierten Rechtsmaterie, die vom Arztrecht über das Gesellschafts- und Arbeitsrecht bis hin zum Steuerrecht reicht", a.a.O., S. 51.

909 S. im Hinblick auf „Eine empirische Analyse der MVZ am Krankenhaus“ ausführlich Hansen, in: Klauber/Robra/Schellschmidt, Krankenhaus-Report 2008/2009 (2009), S. 35 ff.

910 www.kbv.de (Fachbesucher, Themen, MVZ); Stand: 3. Quartal 2008.

911 Dazu insgesamt die Beiträge in: Klauber/Robra/Schellschmidt, Krankenhaus-Report 2008/2009 (2009).

912 Vgl. II.4.7.

913 Vgl. I.4.4.6. 
Hintergrund der Berufsfreiheit nach Art. 12 Abs. 1 GG zuwider. Über die Inkorporation berufsrechtlicher Standards in das Wettbewerbsrecht schließlich kommt es (mittelbar) zur jedenfalls normhierarchisch nicht aufzulösenden bekannten Kollision zwischen Wettbewerbs- und Sozialversicherungsrecht.

Unproblematisch und damit im Wege des Berufsrechts disziplinarrechtlich oder i.V.m. $\S 4$ Nr. 11 UWG sanktionierbar sind nach $\S 31 \mathrm{MBO}$ unmittelbare Entgelte für die Einweisung von Patienten. Allerdings hat die Praxis zum Streit darüber Anlaß gegeben, ob etwa eine von Krankenhäusern angebotene „Betreuungspauschale“ für die postoperative Nachbetreuung gegen das Zuweisungsverbot verstößt. In einem vom $O L G$ Schleswig-Holstein entschiedenen Fall wurde eine solche Pauschale mittels eines Konsiliarvertrages denjenigen niedergelassenen Ärzten angeboten, die der Klinik regelmäßig oder überdurchschnittlich häufig Patienten zugewiesen hatten. ${ }^{914}$ Das Gericht hat die Zulässigkeit im wesentlichen deshalb verneint, weil für den Arzt die gesetzlich vorgesehenen Vergütungsmöglichkeiten nach EBM 2000 plus bzw. GOÄ bestünden, weil die nachstationäre Behandlung in den Aufgabenbereich des Krankenhauses falle bzw. als Behandlung im Krankenhaus stattzufinden habe und schließlich, weil vertragliche Vergütungsregelungen i.S.d. § 115a SGB V unterlaufen würden und Krankenhäuser Entgelte nicht einfach weitergeben dürften. Darüber hinaus gründete das Gericht sein Urteil auf einen Verstoß gegen das Berufsrecht:

„§ 32 BO [Schleswig-Holstein] soll gerade eine Wettbewerbsverzerrung zwischen Ärzten verhindern, die eintritt, wenn die Entscheidung über die Zuweisung zu einem bestimmten Arzt oder an ein bestimmtes Krankenhaus durch Entgeltzahlung beeinflusst wird.“ 915

Unter dem Gesichtspunkt der besseren Verzahnung zwischen ambulanter und stationärer Versorgung schließlich heißt es:

\begin{abstract}
„Die Verzahnung von ambulanter und stationärer Behandlung dient aber dann nicht den Interessen der Patienten, wenn - wie hier - durch die Zahlung eines Geldbetrages die Entscheidung des Augenarztes beeinflusst wird, wohin er einen Patienten für eine Operation überweist, unabhängig von der Qualität der zu erwartenden Behandlung. “ 916
\end{abstract}

Demgegenüber hat das $O L G$ Düsseldorf in einem ähnlich gelagerten Fall, der ebenfalls einen Vertrag mit Entgeltung prä- bzw. postoperativer Leistungen bei Kataraktoperationen betraf, anders, nämlich im Sinne der Zulässigkeit bzw. negativ über einen Unterlassungsanspruch gegen das Krankenhaus betreffend das Angebot entsprechender Kooperationsvereinbarungen entschieden. ${ }^{917}$ Dabei fällt zunächst auf, daß sich das Gericht auf das bereits im Rahmen der Erläuterung des Berufs- und Werberechts beschrie-

914 OLG Schleswig-Holstein, Urt, v. 4.11.2003, MedR 2004, S. 270 ff.

915 A.a.O., S. 274.

916 A.a.O., S. 274.

917 OLG Düsseldorf, Urt, v. 16.11.2004, MedR 2005, S. 169 ff. 
bene „Klinikprivileg“ zurückzieht, demzufolge eine Zurechnung918 an das Krankenhaus ausscheidet ${ }^{919}$ bzw. geringere Maßstäbe anzulegen sind. Zutreffend aber ist von anderer Seite darauf hingewiesen worden, daß die Ärzte an das Gebot gebunden bleiben. ${ }^{920} \mathrm{Da}$ die Frage im Fall also „dahinstehen“ 921 konnte, ändert nichts an einer für die Zulässigkeit erforderlichen Gesamtbetrachtung, die das Berufsrecht mit einbezieht. Dies aber hatte das Gericht nach eigener Anschauung ebenso wenig zu berücksichtigen wie es Ausführungen zu möglichen Verstößen gegen die sozialrechtlichen Bestimmungen (ggf. i.V.m. § 4 Nr. 11 UWG) machte. Es bringt überdies zum Ausdruck, daß im Zusammenhang mit der Vergütungsvereinbarung eine (billigenswerte) Abgeltung der Qualitätssicherung (Dokumentation) erfolgte. ${ }^{922}$

\subsubsection{Sozialrechtliches Selektivvertragsrecht versus Berufsrecht?}

Beide Fälle stehen Pate für die grundsätzliche Problematik des Verhältnisses zwischen Berufsrecht und sozialrechtlichem Selektivvertragsrecht, insbesondere im Bereich der IV. Die scheinbar schlichte Unterscheidung zwischen neuen Versorgungsformen und „Ein- oder Zuweiserbindungen“923 wird hier nicht weiterführen, geht es doch gerade um die weitere Konturierung der Verbotstatbestände im Zeichen ,innovativer“ Versorgungsformen und das Verhältnis der Regime zueinander in den näher zu beschreibenden Konfliktfällen. Im Rahmen der Rabattverträge nach § 130a Abs. 8 SGB V stellen sich in Anbetracht der vielfach organisierten Beteiligung der Ärzte an Einsparungen (in separaten Verträgen) ähnliche Probleme.

In diesem Sinne wird gefolgert, daß Pauschalentgelte der in den vorgenannten Gerichtsentscheidungen beschriebenen Formen im Bereich der IV weniger kritisch zu sehen sind als im übrigen. ${ }^{924}$ Es bleibt allerdings bei der Aufgabe, die „Öffnung des Berufsrechts für das Selektivvertragsrecht“ interpretatorisch zu begleiten: kollisionsspezifisch, teleologisch, verfassungsrechtlich und normhierachisch (i.̈̈.). Weitergehend enthält Art. 11 Abs. 1 S. 2 ApoG ${ }^{925}$ eine (formell-)gesetzliche Kollisionsregel zugunsten des IV-Vertragsrechts mit Blick auf das Ärztebevorzugungsverbot. Möglicherweise kommt dem indiziell eine gewisse Bedeutung für rechtspolitisch avisierte „Öffnungsklauseln“ zu.

918 Diese (i.S.e. Verschuldens) wurde überdies gerade für den vorliegenden Fall verneint, daß „sich eine Krankenhaus bei der Vertragsgestaltung an den Vorgaben eines Vertragsmodells einer Kassenärztlichen Vereinigung“ ”..., „orientiert“, a.a.O., LS. 2.

919 A.a.O., Rn. 170 f.

920 Walter, Pauschalentgelte von Krankenhäusern an niedergelassene Ärzte - zulässige Zuweiserbindung oder berufswidrige Provision?, KHuR 2006, S. 71 (74).

921 OLG Düsseldorf, Urt, v. 16.11.2004, MedR 2005, S. 169 (171 f.).

922 OLG Düsseldorf, a.a.O.

923 Siehe Ratzel, in: ders./Lippert, Kommentar zur MBO, 4. Aufl. (2006), § 31 Rn. 22.

924 Walter, Pauschalentgelte von Krankenhäusern an niedergelassene Ärzte - zulässige Zuweiserbindung oder berufswidrige Provision?, KHuR 2006, S. 71 (76).

925 Dazu Walter, a.a.O., S. 75 f. 


\subsubsection{Vereinbarungen/Absprachen zwischen den Krankenhäusern}

Das nationale ebenso wie das europäische Kartellrecht bilden den Maßstab für kollusives Zusammenwirken der Krankenhäuser als Unternehmen, durch welches die Freiheit des Wettbewerbs um Versorgungsverträge beeinträchtigt wird. Bei entsprechendem Bezug ist auch das europäische Recht einschlägig, etwa betreffend die Gesundheitsversorgung in Grenzregionen, z.B. für Patienten aus anderen Mitgliedstaaten, oder mit Blick auf Leistungserbringer im grenznahen In- und Ausland. Auf der - im Zeichen der Grundfreiheiten weithin deklaratorischen - Grundlage des § 140e SGB V, 926 neu gefasst durch das GKV-WSG, dürfen die Krankenkassen Verträge mit Leistungserbringern 927 in Staaten schließen, in denen die Verordnung (EWG) 1408/71 - in ihrer jeweiligen Fassung - anzuwenden ist.

Seitens der Krankenhäuser etwa vorgenommene Absprachen über die Aufteilung von Abteilungen oder die Patientenzufuhr ${ }^{928}$ sind ebenso wie solche über die (Nicht-) Beteiligung an der IV ${ }^{929}$ am Kartellverbot gem. Art. 81 EGV bzw. § 1 GWB zu messen. Im Verhältnis der Krankenhäuser untereinander oder zu anderen Leistungserbringer, das nicht in, sondern ggf. gegen die Erfüllung der versorgungsvertraglichen Beziehungen zu den Krankenkassen gerichtet ist, ist die Anwendung des $\S 1$ GWB nicht durch $\S 69$ SGB V ausgeschlossen.

Des weiteren kämen im Zeichen der Bildung von und der Konkurrenz um „horizontale(n) Vernetzungen“930 vielfältig kartellwidrige Absprachen in Betracht. Dabei ist im Lichte des $\S 140 b$ SGB V für IV-Modelle allerdings zu berücksichtigen, daß Gemeinschaften aus Vertragspartnern gerade intendiert sind, da „Zusammenschlüsse“ Integrationsleistungen hervorbringen; Kooperation ist hier Strukturmerkmal. Das darf allerdings nicht zu über die IV ausgreifenden kartell- oder wettbewerbswidrigen Absprachen führen. 931

Im übrigen ist bei hinreichender (gesellschaftsrechtlicher) „Integrationsdichte“ auch an kartellwidrige Beschlüsse von Unternehmensvereinigungen oder Marktmißbrauch zu denken. Die Untersagungsbeschlüsse in der Fusionskontrolle haben gezeigt, daß die Märkte der Gesundheitsversorgung in relativer räumlicher Begrenzung zu ermitteln

926 Die Vorschrift beschreibt keinen eigenen Vertragstyp, sondern ist in Kombination mit entsprechenden Regelungen, z.B. i.V.m. § 140a ff. SGB V, anzuwenden; Sichert, Gemeinschaftsrechtliche Vorgaben für Integrierte Versorgung, GuS 2007, S. 28.

927 Welche die Anforderungen des $\S 13$ Abs. 4 S. 2 SGB V bzw. die sekundärrechtlich geprägten Maßstäbe zur Anerkennung von Qualifikation und Zugang zur Berufsausübung erfüllen; s. Sichert, a.a.O., S. 30.

928 Klaue, in: Bruckenberger/Klaue/Schwintowski, Krankenhausmärkte zwischen Wettbewerb und Regulierung (2006), S. 172.

929 Zum Mittelstandskartell mit Blick auf $\S 3$ GWB Bohle, Die Bedeutung des Kartell- und Wettbewerbsrechts bei Krankenhausfusionen, MedR 2006, S. 259 (264).

930 Schwintowski, in: Bruckenberger/Klaue/Schwintowski, Krankenhausmärkte zwischen Wettbewerb und Regulierung (2006), S. 128.

931 Kamann/Gey, Wettbewerbsrecht im deutschen Gesundheitswesen, PhamR 2006, S. 255; 291 (294). 
sind, und so kann es in diesen Räumen schnell zur Marktbeherrschung durch dominierende Leistungserbringer, insbesondere die für die transsektorale Versorgung bedeutsamen Krankenhäuser kommen. Hinsichtlich dieser insofern marktbeherrschenden Leistungserbringer(gesellschaften) ist daher besonderes Augenmerk auf missbräuchliches Verhalten i.S.d. Art. 82 EGV zu legen, insbesondere im Hinblick auf das Diktat marktabschottender Exklusivitätsvereinbarungen als Behinderungsmißbrauch. ${ }^{932}$

\subsection{Der Wettbewerb um Kooperationsgemeinschaften: Die „Binnenkooperation“ mit anderen Leistungserbringern (,, Vorab-Integration“)}

Im Wettbewerb um Selektivverträge mit den Krankenkassen 933 sind viele Leistungserbringer bemüht, sich aus eigenem Antrieb oder im Zusammenhang mit konkreten Vorhaben der Kassen zunächst um erfolgreiche Kooperationsstrukturen mit anderen, potenten Leistungserbringern zu bemühen. In diesem Wettbewerb um Kooperationsgemeinschaften zur „Binnenkooperation“ oder auch „Vorab-Integration“ geht es darum, sich für den direkten Beteiligungswettbewerb gut aufzustellen, strategisch (durch komplementäre ,assets") zu ergänzen und Synergieeffekte vorzugeben. Dadurch kann den Krankenkassen ein abgestimmtes, gemeinsames Versorgungsangebot unterbreitet bzw. vorgezeichnet oder auf Angebote reagiert werden. Krankenhäuser v.a. derselben Versorgungs- und "Spezialisierungsstufe“ konkurrieren damit um Leistungserbringer als Partner, um sich gemeinsam gegen andere - einzeln oder ihrerseits kooperativ agierende - „Anbietergemeinschaften“ durchzusetzen. Entsprechend der Krankenhausdichte sind entsprechende Wettbewerbssituationen v.a. in Städten, z.B. zur Durchführung von DMP denkbar. Bei der Binnenkooperation kann es sowohl zu projektbezogenen Zusammenschlüssen kommen, die zweckgerichtet auf die IV beschränkt und z.B. als „Netzwerkpartnervertrag“ 934 ausgestaltet sind, als auch zu solchen, die im gleichen Zuge eine grundsätzliche und dauerhafte Neuorganisation anstreben. Somit kann sich der Wettbewerb bei der IV auch auf die Landschaft der Leistungserbringer insgesamt auswirken.

Die Krankenhäuser jedenfalls kennzeichnen besondere Attraktivität und Stärke. Dies gilt zum einen wegen ihrer Managementkompetenz, vor allem aber wegen ihrer Unentbehrlichkeit zum finanzierungserheblichen Integrationsverbund mit dem stationären Sektor nach $\S 140 \mathrm{a}$ ff. SGB V, auch wenn damit nur ein (leistungsrechtlich weiter konkretisierungsbedürftiger) Teilausschnitt der IV beschrieben ist. Auch in diesem Zusammenhang ist die Trägerschaft von MVZ durch Krankenhausträger von Bedeutung. Selbst wenn Krankenhäuser das Know-how für die ambulante ärztliche Versorgung in der Regel „einkaufen“ müssen, so haben sie doch besondere Erfahrung in Sachen des Betreibens von Einrichtungen, entweder selbst oder im Sinne des „Out“ bzw. „Insour-

932 Kamann/Gey, a.a.O.

933 Vgl. II.3.1.2.

934 Vgl. Wallhäuser, Verträge in der Integrierten Versorgung (2005), S. 29 ff., 51 ff. 
cing“ mittels Betreibergesellschaften. Kommt darüber hinaus die Gründung einer Managementgesellschaft in Frage, können sich Krankenhäuser zur Sicherstellung der Einflussmöglichkeiten an diesen beteiligen. Nimmt man Out- und Insourcing zusammen, wobei unter „Insourcing“ fachintern die Übertragung von Aufgaben an ein vom Krankenhaus oder dem Träger gegründetes Unternehmen verstanden wird, haben nach der Umfrage 2007 (Krankenhaus Barometer) 8 Prozent der Häuser sowohl In- als auch Outsourcing betrieben. 935

Das Bild der Kooperationen allerdings ist erst vollständig, wenn man auch die Kooperationen der Krankenhäuser miteinander berücksichtigt. „Knapp die Hälfte der Krankenhäuser (48\%) ist seit dem Jahr 2004 eine institutionalisierte Form von Kooperationen mit anderen Krankenhäusern eingegangen“, die „am häufigsten... im Bereich der medizinischen Kernleistungen und Funktionsbereiche (wie z.B. Labor, Radiologie)“ stattfinden. 936

\subsection{Verdrängungswettbewerb: Konzentrationsprozeß und Gemeinwohlaufgaben}

\subsubsection{Entwicklungstendenzen}

Die vorgenannten Aspekte lassen erahnen, daß zunehmende Konzentrationsprozesse die Sicherstellung der „,bedarfsgerechten Versorgung der Bevölkerung“ ( $§ 1$ Abs. 1 KHG) vor besonderen Herausforderungen stellt. Das gilt umso mehr, als ein Qualitätsund Spezialisierungswettbewerb in Anbetracht begrenzter „Nachfragekapazitäten“ auch als Verdrängungswettbewerb zum Ausdruck kommt. Die (möglichen) Folgen im Zuge eines immer stärkeren Konzentrationsprozesses bzw. einer abnehmenden Zahl der Krankenhäuser sind bekannt; es bestehen zunehmend Defizite hinsichtlich der Versorgung in der Fläche. Für die Notfallversorgung wiederum ergeben sich zudem die im Zusammenhang mit der strukturellen Reorganisation der Krankenhäuser (4.7.1) zu beschreibenden Effekte. Dies bestärkt auch die „Konzentration der Notfallversorgung auf wenige Kompetenzzentren, was für die Patienten zum Teil lange Transportzeiten erforderlich mache“.937

Problematisch ist also, wie diesem Befund zumindest teilweise steuernd entgegengewirkt werden kann. Prospektiv betrachtet könnten insoweit Finanzierungsanreize gewährt werden, sei es als Entgeltbestandteil oder -zuschlag oder qua Ausgleichsfinanzierung durch ein Fondsmodell. ${ }^{938}$ Aktuell jedenfalls scheint wenig Erfolg versprechend, (gerade) die flächendeckend stationäre Versorgung durch IV-Modelle zu betreiben, um

935 DKI (Hrsg.), Krankenhaus Barometer, Umfrage 2007, S. 26 f.

936 A.a.O., S. 29 f.

937 S. die Meldung „Ist die flächendeckende Notfallversorgung in Bayern in Gefahr?“, Ärzte Zeitung, 21.10.2002 (www.aerztezeitung.de).

938 S. Ebsen, Perspektiven der Krankenhausplanung in einem gewandelten Markt und einem föderalen Gefüge, in: Klauber/Robra/Schellschmidt, Krankenhaus-Report 2006 (2007), S. 117 (129 f.). 
dadurch Infrastrukturprojekte zu fördern. ${ }^{939}$ Allerdings steht zu erwarten und wird auch z.T. verlautbart, daß gerade größere Krankenhausketten im Sinne des nicht zu unterschätzenden Imagewettbewerbs sich auch in der Fläche mit Häusern engagieren. Im Konzernverbund bestehen dazu auch entsprechende Möglichkeiten; zahlreiche „Leistungen“ sind gleichwohl mittels „outsourcing“ organisierbar, wie etwa die externe Röntgendiagnostik bzw. zentrale Auswertung.

\subsubsection{Verlustausgleich}

Dennoch droht oftmals gerade den Häusern, die sich in erster Linie als Einrichtungen zur Daseinsvorsorge verstehen, das wirtschaftliche Aus. Daher gewähren Kommunen ihren Einrichtungen z.T. Ausgleichszahlungen für die Erfüllung von Aufgaben der Daseinsvorsorge. Hier ist bereits darauf hingewiesen worden, daß es einer Notifizierung der Verlustausgleiche nur dann nicht bedarf, wenn die bzgl. Art. 87 Abs. 1 EGV in der Sache Altmark-Trans aufgestellten Kriterien erfüllt sind. ${ }^{940}$

$\mathrm{Ob}$ dies der Fall ist, muß im Einzelfall geprüft werden. Ein nachträglicher Verlustausgleich bzw. ein solcher ohne vorherige Aufstellung objektiver und transparenter Kriterien erfüllt stets den Anwendungsbereich des Art. 87 EGV. ${ }^{941}$ Unbeschadet der Festlegung der Kriterien handelt es sich ,in der Tat... bei den herkömmlichen Ausgleichszahlungen um einen nachträglichen Verlustausgleich“. ${ }^{942} \mathrm{Da}$ die den Tatbestand ausschließenden Altmark-Kriteren allerdings generell nicht erfüllt seien, weil es an einer Aufgabe fehle, „mit welcher gerade die öffentlichen Krankenhäuser betraut worden sind“, 943 kann nicht gesagt werden. Zwar besteht der Versorgungsauftrag der Plankrankenhäuser unabhängig von der Trägerschaft, und Planungs- und kommunaler Krankenhausträger sind auch nicht identisch. Eine derartige Akzessorietät ist den AltmarkKriterien allerdings ebenso wenig zwingend zu entnehmen, wie eine in diese Richtung tendierende Sichtweise den rechtlichen Bedingtheiten der Verankerung der Krankenhausversorgung als kommunaler Pflichtaufgabe nicht gerecht würde. ${ }^{944}$

939 Speziell unter diesem Gesichtspunkt kam die ausdrücklich an einer entsprechenden Zielrichtung orientierte Studie über „Integrierte Versorgung als Baustein für eine moderne Gesundheitswirtschaft in den neuen Bundesländern“" zu negativen Ergebnissen, vgl. Bundesamt für Bauwesen und Raumordnung unter www.brr.bund.de (Navigation: Forschungsprogramme, Aufbau Ost, Infrastruktur).

940 Dazu oben I.4.4.3.2.

941 Siehe auch Cremer, Krankenhausfinanzierung im europarechtlichen Kontext, in: Ministerium für Arbeit, Gesundheit und Soziales des Landes Nordrhein-Westfalen (Hrsg.), Krankenhausrecht: Herausforderungen und Chancen, 2005, S. 23 (36); Vollmöller, Krankenhausfinanzierung und EGBeihilferecht, in: Bauer u.a. (Hrsg.), FS für Reiner Schmidt (2006), S. 205 (209).

942 Vollmöller, a.a.O.

943 Cremer, Krankenhausfinanzierung im europarechtlichen Kontext, in: Ministerium für Arbeit, Gesundheit und Soziales des Landes Nordrhein-Westfalen (Hrsg.), Krankenhausrecht: Herausforderungen und Chancen, 2005, S. 23 (35 f.).

944 Vollmöller, Krankenhausfinanzierung und EG-Beihilferecht, in: Bauer u.a. (Hrsg.), FS für Reiner Schmidt (2006), S. 205 (209). 
Liegt aber (dennoch) eine Beihilfe vor, ist die Ausnahmevorschrift des Art. 86 Abs. 2 EGV zu prüfen, die Unternehmen, die mit Dienstleistungen von allgemeinem wirtschaftlichen Interesse (DAWI) betraut sind, unter bestimmten Voraussetzungen von der Anwendung der Wettbewerbsvorschriften ausnimmt. Nach Art. 16 EGV ist die Durchführung der Aufgaben der DAWI gemeinschaftsrechtlich spezifisch anerkannt. Das neue Protokoll (Nr. 9) 945 über DAWI, das nach dem Entwurf des Änderungsvertrages vom 5. Oktober 2007 dem Vertrag über die Arbeitsweise der Europäischen Union beizufügen ist, dürfte an den hier maßgeblichen Grundsätzen im Ergebnis wenig ändern. ${ }^{946}$ Der Rechtfertigung kommt in der Praxis besondere Bedeutung zu. Im November 2005 erließ die Kommission eine Entscheidung über die Anwendung des Art. 86 Abs. 2 EGV auf staatliche Beihilfen, die bestimmten mit der Erbringung von DAWI betrauten Unternehmen als Ausgleich gewährt wurden, ${ }^{947}$ flankiert von einem Gemeinschaftsrahmen für Beihilfen, die als Ausgleich für die Erbringung öffentlicher Dienstleistungen gewährt werden. ${ }^{948}$ Nach Art. 2 Abs. 1 lit. a der Entscheidung gilt diese auch für „Ausgleichszahlungen an Krankenhäuser“. Im Krankenhaussektor allerdings existieren Besonderheiten. Während nach dem Gemeinschaftsrahmen grundsätzlich eine Notifizierungspflicht bestehen bleibt, ${ }^{949}$ nimmt die Entscheidung u.a. den Krankenhausbereich von der Notifizierung aus. ${ }^{950}$ Die Entscheidung erkennt für den Krankenhaussektor an, 951

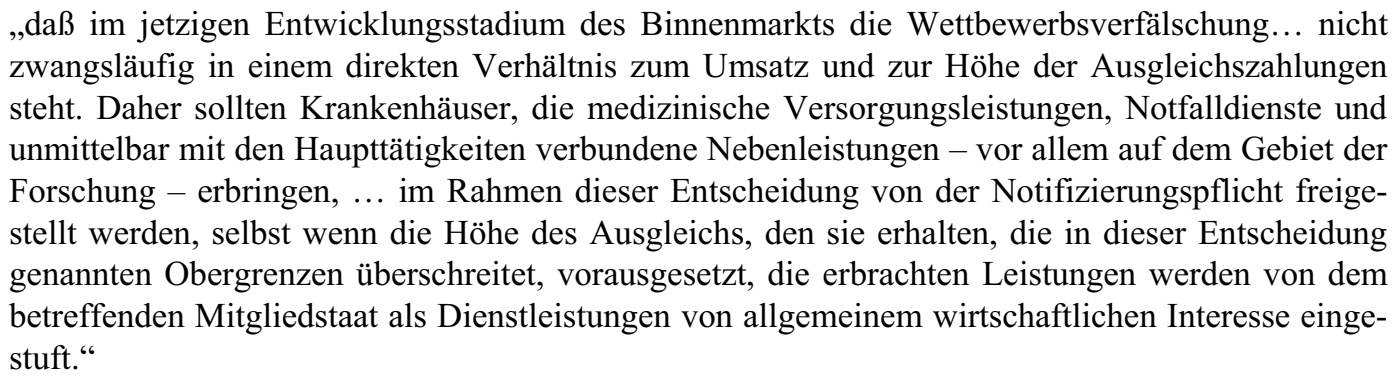

Die Freistellung, vgl. Art. 3 der Entscheidung, setzt allerdings die Erfüllung der dort aufgestellten Voraussetzungen voraus. Sie entsprechen weithin den Kriterien in Sachen Altmark-Trans, wobei das „Monti-Paket“ ein transparentes Verfahren für die Ermittlung der Parameter für die Berechnung, Überwachung und etwaige Änderung der Ausgleichszahlungen nicht erforderlich macht. ${ }^{952}$ Da mangels der Erfüllung der Entscheidungskriterien die Notifizierung aber erforderlich bleibt und die Mitgliedstaaten aufge-

945 CIG 2/1/07 REV 1, S. 25.

946 Sichert, Gemeinschaftsrechtliche Vorgaben für Integrierte Versorgung, GuS 2007, S. 28 (34 f.).

947 2005/842/EG, ABl. EU 2005 Nr. L 312, S. 67.

948 ABl. EU 2005 Nr. C 297, S. 4.

949 Nach Ziff. 2.4. Rn. 16, Ziff. 5 Rn. 25.

950 Vgl. Erwägungsgrund 16, Art. 3 der Entscheidung.

951 A.a.O., Erwägungsgrund 16.

952 Vgl. Art. 4 lit. d der Entscheidung; Ziff. 2.3. Rn. 12 des Gemeinschaftsrahmens; siehe auch Becker, EU-Beihilfenrecht und soziale Dienstleistungen, NZA 2007, S. 169 (175). 
fordert waren, innerhalb von achtzehn Monaten nach Veröffentlichung des Gemeinschaftsrahmens ihre jeweiligen Regelungen hinsichtlich des Ausgleichs für die Erbringung von DAWI mit dem Gemeinschaftsrahmen in Einklang zu bringen, bestand vielerorts Handlungsbedarf. Eine Umfrage seitens des Bayerischen Staatsministeriums für Arbeit und Sozialordnung, Familie und Frauen ergab, daß 38\% von 200 Krankenhäusern mit Verlustausgleichen operierten, vorrangig solche der Grund- und Regelversorgung. ${ }^{953}$ Die Gesamtsumme der Ausgleiche betrug 1,5\% der Budgets aller 200 Häuser, 4,1\% des Budgets der defizitären Krankenhäuser. 954

In Zweifelsfällen ist daher eine Notifizierung angezeigt. Im übrigen besteht die Herausforderung in der Aufstellung der Kriterien für den Verlustausgleich. Soweit dies im Gesamtzusammenhang der Betrauung ,im Wege eines oder mehrerer Verwaltungsoder Rechtsakte“ erfolgen kann, dürfte die „duale Zuständigkeit“" von Planbehörde und kommunalem Träger kein Hindernis darstellen. 955

\subsection{Fusionswettbewerb}

Der Fusionswettbewerb der Krankenhäuser 956 stand bereits mehrfach im Blickpunkt, etwa unter dem Gesichtspunkt der Wahlrechte des Patienten, der Einweisungssteuerung, der Betrachtung von MVZ u.a. „Seit dem Jahr 2004“, so die Umfrage (Krankenhaus Barometer) 2007, „haben 9\% der Krankenhäuser Fusionen mit einem oder mehreren Krankenhäusern vollzogen. Dabei nehmen mit steigender Bettengrößenklasse die Fusionen zu." $" 957$

Die im Bereich der Zusammenschlußkontrolle ergangenen Untersagungsentscheidungen durch das BKartA und die Bestätigung der Entscheidung vom April 2007 durch das $O L G$ Düsseldorf 958 haben den Markt für Krankenhausleistungen in seinen Wirkungsbeziehungen unter normativen Gesichtspunkten näher konturiert. Das gilt insbesondere für den Absatzmarkt und mit Blick auf die Wahlrechte der Patienten. Zugleich wurde die Reichweite der Exklusivitätsbestimmung nach § 69 SGB V zugunsten der Anwendbarkeit der Zusammenschlußkontrolle konkretisiert. Kontrastiert mit den Entscheidungen des $B S G^{959}$ und des $B G H$ zugunsten der materiellen Ausschlußwirkung

953 Knorr, Krankenhausversorgung und EU-Wettbewerbsrecht, Präsentation zur Fachtagung des BKKLandesverbandes Bayern am 12. Juni 2007, Folie 11.

954 A.a.O.

955 Vgl. Vollmöller, Krankenhausfinanzierung und EG-Beihilferecht, in: Bauer u.a. (Hrsg.), FS für Reiner Schmidt (2006), S. 205 (211).

956 Böge, Der Markt für Krankenhausleistungen aus Sicht des Bundeskartellamts, in: Klauber/Robra/ Schellschmidt (Hrsg.), Krankenhausreport 2006 (2007), S. 35 ff.; Bohle, Die Bedeutung des Kartellund Wettbewerbsrechts bei Krankenhausfusionen und Krankenhauskooperationen, MedR 2006, S. $259 \mathrm{ff}$.

957 DKI (Hrsg.), Krankenhaus Barometer, Umfrage 2007, S. 31.

958 S. Fn. 429.

959 BSG 89, 24 (33). 
wettbewerbsrechtlicher Ansprüche, die sich ,auch auf die Beziehungen von Leistungserbringern untereinander“ erstreckt, ,soweit es um Handlungen in Erfüllung des öffentlich-rechtlichen Versorgungsauftrags der Krankenkassen geht" ,960 ist der Zusammenschluss keine der Erfüllung des öffentlich-rechtlichen Versorgungsauftrags dienende Veraltensweise. Dies gilt auch insoweit, als der Konzentrationsprozess im Sinne effizienter Versorgung intendiert ist.

Wie im Rahmen der Wahlrechte des Patienten, aber auch bezüglich der Akquisition stationärer Patienten im Kontext ambulanter Versorgung (MVZ) zum Ausdruck kam, sind den Krankenhäusern erhebliche Verhaltensspielräume attestiert worden. Auch angesichts dessen kam ein Ausschluß des GWB danach nicht in Betracht. ${ }^{961}$ Bei alledem aber ist zu bedenken, daß das BKartA ein Zusammenschlußvorhaben bisher allein in 2 von mindestens 50 geprüften Fällen mit Blick auf $\S 36$ Abs. 1 GWB untersagt hat, weil die Akquisition die martktbeherrschende Stellung des erwerbenden Klinikums auf dem Markt für Krankenhausleistungen im räumlich relevanten Markt verstärkt hätte. 962

Der Konzentrationsprozeß wird durch die organisationsspezifischen Grunddaten moderat abgebildet: Die Zahl der Krankenhäuser hat in den Jahren 2000-2007 um über 150, die Zahl der aufgestellten Betten um mehr als 52.000 abgenommen. ${ }^{963}$ Die Zahl der Betten je Einrichtungen blieb dennoch im wesentlichen gleich (250/243). Veränderungen gibt es hier bezogen auf die ab 2002 registrierten Angaben zu den noch in öffentlich-rechtlicher Form geführten öffentlichen Einrichtungen, bei denen entgegen gleichbleibender Zahl bis 2006 ein Zuwachs um 21 Betten im Folgejahr zu verzeichnen war, sowie - als Ausdruck eines kontinuierlichen stetigen Anstiegs - bei den privaten Einrichtungen ( 35 gegenüber 2002).

\subsection{Wettbewerbsdruck und strukturelle Reorganisation}

Die vorgenannten wettbewerbsorientierten Entwicklungen erweisen sich als Katalysatoren vielfältiger Tendenzen der strukturellen Reorganisation der Krankenhäuser. Sie betreffen in erster Linie die Organisationsform - bis hin zur materiellen Privatisierung das Leistungsangebot und das Versorgungsmanagement. Sie sind einerseits Ausdruck und Folge, andererseits Kennzeichen einer Neuaufstellung, die wettbewerbsorientiertes Verhalten ermöglicht und beflügelt. Insbesondere der seitens des G-DRG-Systems ausgelöste Spezialisierungsdruck führt ebenso wie der Ausschluß der Möglichkeiten zur Entgeltgestaltung durch das regulierte Preissystem zu verstärkten Bestrebungen struktureller Reorganisation.

960 BGH Urt. v. 23.2.2006, GRUR 2006, S. 517 ff. = NJW RR 2006, S. 1046 ff.

961 A.a.O., Ziff. $48 \mathrm{ff}$.

962 A.a.O., a.a.O., zusammenfassend Ziff. 187.

963 StBA, Grunddaten der Krankenhäuser 2007 (2008), Allgemeiner Überblick 2007, sowie Ziff. I. 1.4. Näher zu den Strukturdaten unter I.3.2.; s. auch II.4.7.4. 


\subsubsection{Allgemeine Tendenzen, Zentrenbildung und Bedeutung für die Notfallversorgung}

Viele öffentliche Krankenhäuser haben durch die Wahl privatrechtlicher Rechtsformen neue Strukturen für ein effizienteres Management geschaffen, 964 wie etwa die „kommunale Krankenhaus-GmbH“.965 Weitergehend wird mitunter auch ein Wechsel in private Trägerschaft vollzogen. ${ }^{966}$ Beides hat freilich nicht das Ausscheiden aus dem Krankenhausplan zur Folge. Im Zuge struktureller Reorganisation kann es dazu kommen, daß sich das Krankenhaus neu aufstellt und im Rahmen einer nunmehr niedrigeren Versorgungsstufe oder durch Schließungen von Fachabteilungen vormals wahrgenommene Aufgaben der Notfallversorgung nicht mehr erfüllen kann; dabei folgt die generelle Verpflichtung zur Notfallversorgung für Plankrankenhäuser aus den Landeskrankenhausgesetzen i.V.m. den (allgemeinen) Bestimmungen in den Krankenhausplänen. Ausweislich der jüngeren Umfrage des DKI 2007 hat nach der verbindlichen Einführung der DRGs im Jahr 2004 ,rund ein Fünftel der Krankenhäuser mindestens eine Fachabteilung neu errichtet.“967 Zugleich aber wird berichtet, daß Fachabteilungen verstärkt aufgelöst worden sind. 968

Die „Konzentration auf die eigenen Stärken“ als „Möglichkeit zur Stärkung der wettbewerblichen Position bei gleichzeitiger Wahrung oder Verbesserung der medizinischen Leistungsfähigkeit der Krankenhäuser“ ist die Bildung von Zentren. ${ }^{969}$ Man mag dies zunächst institutionell im Hinblick auf die Bildung und Steuerung mit Hilfe von $\mathrm{MVZ}^{970}$ oder auch im Hinblick auf „Zentren und Schwerpunkte“ im Sinne von $\S 2$ Abs. 2 S. 2 Nr. 4 KHEntgG verstehen. Für letztere indes werden unbeschadet der Problematik einer akkuraten Bestimmung der Zugehörigkeit „,weitgehend inhaltsfreie Vorgaben zur Bestimmung einer Vergütung" konstatiert. ${ }^{971}$ Jedenfalls sind die Vergütungen, insbesondere Zuschläge, nach $\S 17 \mathrm{~b}$ Abs. 1 S. 4 KHG i.V.m. § 11 KHEntgG von den Vertragsparteien (nach $\S 18$ Abs. 2 KHG) zu regeln. 972 Der ökonomische Vorteil ist insoweit nicht garantiert. Ungeachtet dessen, d.h. jenseits „,besonderer Leistungen“, kann eine zentrierte Versorgung v.a. als ,konzentrierte“, spezialisierte Versorgung stattfinden, die sich an den (allgemeinen) Maßstäben des Angebotswettbewerbs ausrich-

964 Vgl. oben I.3.2.2.1. Näher noch unter II.4.7.4.

965 Quaas, in: Wenzel (Hrsg.), Handbuch des Fachanwalts Medizinrecht (2007), Kap. 12 Rn. 21.

966 Vgl. das Zusammenschlußvorhaben der Rhön-Klinikum AG mit den Krankenhäusern des Landkreises Rhön-Grabfeld, BKartA, Beschl. B 10 - 123/04.

967 DKI (Hrsg.), Krankenhaus Barometer, Umfrage 2007, S. 25.

968 A.a.O.

969 Klauber/Robra/Schellschmidt, Krankenhaus-Report 2008/2009 (2009), Schwerpunkt: Versorgungszentren, Vorwort.

970 Vgl. II.4.3.1.

971 Deh/Dralle, in: Klauber/Robra/Schellschmidt, Krankenhaus-Report 2008/2009 (2009), S. 61 (65).

972 A.a.O., S. $63 \mathrm{ff}$. 
tet. 973 Des weiteren lassen sich Zentrumsstrukturen ausmachen, die „output-orientiert“, d.h. an spezifisch zentrierter Leistungserbringung und nicht fachbezogen funktionsorientiert sind. ${ }^{974}$ Dies lässt sich letztlich wohl so begreifen, daß von einer ,produktorientierten" Optimierung derart auszugehen ist, daß die Organisationsstruktur der integrierten Leistungsstruktur folgt. Man könnte ggf. auch von organisationsstruktureller Binnenintegration und -konzentration sprechen. Damit verbunden jedenfalls ist eine Spezialisierung, und die Definition von Behandlungspfaden wird erleichtert. 975

Der Spezialisierungswettbewerb schließlich hat zu massiven Befürchtungen geführt, ineffiziente Strukturen vor allem der Notfallversorgung einschließlich der vorzuhaltenden Kapazitäten abzubauen, da eine Refinanzierung durch DRG nicht kostendeckend, eine Quersubventionierung hingegen nicht zulässig sei. Das gilt insbesondere im Hinblick auf chirurgisch zu versorgende Polytraumata, bzgl. derer im Jahre 2004 „,bei jährlich etwa 25.000 Schwerverletzten ... voraussichtlich dramatische Auswirkungen auf die ... Schwerstverletztenversorgung“" prophezeit wurden. ${ }^{976}$ Die Kapazität zur Akutversorgung entfällt überdies gänzlich, wenn die Krankenhäuser - wie in Bayern 22 Häuser in den Jahren von 1990 bis 2002 - aus dem Krankenhausplan ausscheiden. ${ }^{977}$ „Reine Privatkliniken“ zur exklusiven Behandlung selbstzahlender Patienten werden nicht in den Plan aufgenommen und verfügen über keinen Versorgungsauftrag nach $\S \S 108$ Nr. 3, 109 SGB V. ${ }^{978}$ Als regulierendes Korrektiv allerdings rückt $\S 17 \mathrm{~b}$ Abs. 1 S. 4 KHG in den Vordergrund; nach der entsprechenden Vereinbarung, § 2, gilt: Krankenhäuser, die nicht an der stationären Notfallversorgung teilnehmen, erhalten einen Abschlag vom Basisfallwert. ${ }^{979}$

\subsubsection{Symbiose von Plankrankenhaus und gewerblicher Privatklinik}

Vor diesem Hintergrund stellt sich das Problem der Zulässigkeit organisatorischer Verschränkungen, wenn das öffentliche bzw. Plankrankenhaus die Strategie einer finanziellen Sanierung durch das Angebot „rein privatrechtlich“ abzurechnender Leistungen verfolgt und zu deren Realisierung auf Einheiten einer ,integrierten“ oder kooperierenden Privatklinik zurückgreift. Ziel ist es hier, wie bei selbständigen reinen Privatpatientenkliniken auch, ohne Bindung und Beschränkung an KHG, BPflV und KHEntgG Erlöse zu erwirtschaften. Diese Strategien sind nicht auf die Erbringung von Wahlleistun-

973 Vgl. II.4.2.

974 Kuntz/Wittland, in: Klauber/Robra/Schellschmidt, Krankenhaus-Report 2008/2009 (2009), S. 87 (88 ff.).

975 A.a.O.

976 Siehe Deutsche Gesellschaft für Unfallchirurgie, DRG-News, 25.8.2004, abrufbar unter www.dguonline.de.

977 Siehe die Meldung „Ist die flächendeckende Notfallversorgung in Bayern in Gefahr?“, Ärzte Zeitung, 21.10.2002 (www.aerztezeitung.de).

978 Quaas, sind Wahlleistungen und Privatkliniken eine finanzierungsrechtliche Alternative?, KHuR 2006, S. 114 (119 f.).

979 Siehe unter http://www.g-drg.de/systemgrundlagen/drg_verzuab.php?m=3. 
gen gerichtet, welche die allgemeinen Krankenhausleistungen ergänzen, sondern es geht darum, das gesamte Leistungsspektrum des Krankenhauses ,,außerhalb des Sozialleistungsrechts und Pflegesatzrechts berechnen zu können." 980 Solche Vorhaben werden durch verschiedene Konzepte verfolgt, wobei die ersten beiden der sogleich zu benennenden dominieren: 981

- Neugründungen gewerblicher Privatkliniken ( $\$ 30 \mathrm{GewO}$ ) und Angliederung an das öffentliche Krankenhaus; dabei werden die Unterkunftsleistungen mit eigenen Mitteln der Privatklinik erbracht und die Behandlungsleistungen beim Plankrankenhaus „,eingekauft“; 982

- Ausgründungen von Bereichen zur Behandlung selbst zahlender Privatpatienten aus dem öffentlichen Krankenhaus;

- Einbeziehung bestehender Privatkliniken durch Übernahme, Beteiligung oder Kooperation.

„Wenn ... der Betrieb eines Plankrankenhauses und einer Privatklinik ... in derselben Stadt zulässig ist", so fragt Quaas, ,warum soll dann nicht das »Krankenhaus im Krankenhaus« bzw. der Betrieb zweier Krankenhäuser »unter einem Dach« möglich sein?"983

Der Kern dieser Fragestellung ist nicht in erster Linie ein organisationsrechtlicher, sondern betrifft die Auswirkungen auf bzw. Folgen nach Krankenhausplanungsrecht sowie die Befugnis zur Abrechnung außerhalb der für die öffentlich-rechtlichen Bestimmungen. Daß die Verschränkung Auswirkungen auf die Krankenhausplanung haben kann, so insbesondere hinsichtlich der Festlegung des Bettenangebotes des Hauses bzw. die Reduktion der Fördermittel auf den spezifisch planerischen - auf die allgemeine Bevölkerung bezogenen 984 - Bereich, ist nicht zu bestreiten. ${ }^{985}$ Fraglich ist nur, ob dies zur Unzulässigkeit entsprechender organisatorischer Abrechnungseinheiten führt, 986 wenn die Vorgaben im übrigen beachtet und Fördermittel ggf. zurückgefordert werden. Unter Beachtung der Folgen für die Planung aber ist gegen die Symbiose nichts einzuwenden, auch wenn man sich insofern fragen mag, ob damit nicht maßgebliche Vorteile des Konzepts überhaupt entfallen.

980 Quaas, sind Wahlleistungen und Privatkliniken eine finanzierungsrechtliche Alternative?, KHuR 2006, S. 114 (119).

981 Patt, in: Uleer/Miebach/Patt, Abrechnung von Arzt- und Krankenhausleistungen, 3. Aufl. 2006, 2. Teil A Rn. 71.

982 Siehe auch Quaas, Sind Wahlleistungen und Privatkliniken eine finanzierungsrechtliche Alternative?, KHuR 2006, S. 114 (120).

983 A.a.O.

984 Dazu gehören - mit der Folge der Bindung an das Finanzierungsrecht - auch Privatpatienten, Patt, in: Uleer/Miebach/Patt, Abrechnung von Arzt- und Krankenhausleistungen, 3. Aufl. 2006, 2. Teil A Rn. 74.

985 Vgl. Quaas, Sind Wahlleistungen und Privatkliniken eine finanzierungsrechtliche Alternative?, KHuR 2006, S. 114 (120).

986 So Patt, in: Uleer/Miebach/Patt, Abrechnung von Arzt- und Krankenhausleistungen, 3. Aufl. 2006, 2. Teil A Rn. 73 f. 
Sinn und Zweck solcher Strategien erweisen sich danach bei Beantwortung der Frage, ob bei entsprechender organisatorischer Verschränkung von den öffentlichen Finanzierungsvorschriften abgewichen werden darf. Die dazu vertretenen Auffassungen sind konträr. ${ }^{987}$ Die Beurteilung über das Vorliegen zahlreicher Verstöße gegen das KHG (§ 1 Abs. 1, § 6 Abs. , § 17 Abs. 1) hängt letztlich davon ab, ob rechtstatsächlich eine wirtschaftlich und organisatorisch einheitliche Betrachtungsweise angezeigt ist oder nicht, bzw. ob darüber hinaus Art. 12 GG im Sinne einer Trennungsthese den entsprechenden Betrieb der Privatklinik ebenso wie deren Errichtung schützt, bis hin zur Verhinderung eines Erlösausgleichs. ${ }^{988}$ Allerdings ist zu bedenken, daß Freiheit nicht a priori das Recht erfaßt, Strukturvorteile aus der Planung einzubringen, wenn die Planungsziele, d.h. vor allem die Versorgung der - gesamten - Bevölkerung durch Ausgliederung oder Neugründung, bewusst negiert werden. Nicht die „Privatisierung“ ist unzulässig, wohl aber das planwidrige (Mit-) Betreiben mittels der ,geplanten“" Versorgungseinheit.

Über den Erfolg derartiger Strategien schließlich ist bislang wenig bekannt. Im übrigen ist darauf hinzuweisen, daß entsprechende Möglichkeiten zahlungskräftige Patienten voraussetzen. Insoweit und bereits mit Blick auf medizinische Wahlleistungen tritt die private Krankenversicherung nur bei entsprechender Vereinbarung bzw. für den nach $\S 1 \mathrm{MB} / \mathrm{KK}$ beschriebenen Versicherungsfall der medizinisch notwendigen Heilbehandlung wegen Krankheit oder Unfallfolgen ein.

\subsubsection{Case- und Personalmanagement}

Ein Schwerpunkt der in Folge wettbewerbsorientierter Strukturen indizierten krankenhausinternen strukturellen Reorganisation liegt auch im Bereich des CaseManagements durch eigens dafür zuständiges (und ausgebildetes) nichtärztliches (Pflege-)Personal. ${ }^{989}$ Sie führt zur Entlastung der Ärzte von betriebswirtschaftlich relevanten Optimierungsplanungen sowie zur spezifischen Professionalisierung dieser Art der Tätigkeit. Die Aufwertung des Case Managements korrespondiert mit dem durch das GKV-WSG neu geschaffenen $§ 11$ Abs. 4 S. 1 SGB V, der bestimmt: „Versicherte haben Anspruch auf Versorgungsmanagement...". Unbeschadet der Entlastung des Arztes hat diese Neuausrichtung unter dem Gesichtspunkt der Ausführung durch nichtärztliches (Pflege-)Personal allerdings unverkennbar Konsequenzen für das Berufs- und Tätigkeitsfeld der Heilberufe. Ohne Zweifel „,verantwortet“ der Case Manager medizinisch relevante und medizinisch zu fundierende Entscheidungen. Während eine spezifische Revision der Ausbildung - und entsprechende Besoldung - tendenziell leichter zu erzielen ist, sind Haftungs- und Zurechnungsfragen noch weithin ungeklärt. Das gilt auch für das Berufsrecht.

987 Für Unzulässigkeit Patt, a.a.O., Rn. 71 ff.; a.A. Quaas, Sind Wahlleistungen und Privatkliniken eine finanzierungsrechtliche Alternative?, KHuR 2006, S. 114 (120 f.).

988 Quaas, a.a.O.

989 Vgl. von Rechenberg, Case-Manager: Wenn die Schwester den Arzt ersetzt (s. Literatur); Löcherbach, Ausbildung zur Case Managerin bzw. zum Case Manager in Deutschland (s. Literatur). 
Darüber hinaus zeigt sich in der jüngeren Umfrage (Krankenhaus Barometer) 2007, daß die Fachwelt $\S 11$ Abs. 4 SGB V als normative Basis für ein umfassendes Entlassungsmanagement betrachtet, das schriftliche Standards, die Bereitstellung von Fachkräften, die Organisation des Entlassungsmanagements sowie die Einbeziehung von Nachsorgern in die Entlassungsplanung beinhaltet. ${ }^{990}$ Ferner werden an das Case Management unter neuen Strukturen höchste Erwartungen geknüpft, wie Einschätzungen zur Erzielung von Effizienzreserven im Umfang von 1,5 Stunden Arbeitszeit/Tag des Arztes bei gleichzeitiger Patientenzufriedenheit über die raschere und personenorientierte Abläufe illustrieren. ${ }^{991}$ Letzteres ist nicht allein kostenrelevant, sondern auch mit Anreizen für die Wahl des Krankenhauses durch den Patienten.

Ohne Zweifel schließlich stellen DRG und integrierte Systeme mannigfache Herausforderungen auch an das Personalmanagement; 992 diesbezüglich allerdings sind im wesentlichen Fragen der Governance und der Unternehmenspolitik in Bezug genommen, die hier - unbeschadet einzelner juristischer Implikationen - aus der Perspektive normativer Steuerung im Sinne der Untersuchung nicht weiter verfolgt werden können.

\subsubsection{Grunddaten der Krankenhäuser: Indikatoren struktureller Reorganisation? ${ }^{993}$}

Aus juristischer Sicht erscheint es problematisch, die Auswirkung der Effekte normativer Steuerung mittels der Grunddaten der Krankenhäuser veritabel messen zu wollen. Wie bereits angedeutet, sind diese Effekte in einem Kausalitäts- und Beziehungsgeflecht zu würdigen, wobei „wettbewerbsverzerrende“ regulatorische Steuerungsansätze ebenso einzubeziehen sind. Danach waren eher einzelne Vertrags-, Einweisungs- und Finanzierungsstrategien und ihre Einbindung in eine Regulierungsverflechtung zu identifizieren.

Aus normativer Warte sind bereits die Maßstäbe einer Bewertung und deren relative Gewichtung zu hinterfragen. So mögen der Konzentrationsprozeß und die Senkung der Verweildauer Ausdruck allgemein funktionierenden Wettbewerbs sein, ohne daß damit Aussagen über die Qualität der Behandlung verbunden sind. Die Verweildauer - im Hinblick auf die sog. „,blutige Entlassung“ oftmals kritisch betrachtet - besagt ebenfalls wenig über die Qualität der Behandlung. „Qualität“ wiederum ist auch ein Rechtsbegriff, müßte zudem im Hinblick auf Struktur-, Prozeß- und Ergebnisqualität unterschieden werden. Die Sicherstellung der Versorgung schließlich hat nicht nur finanzielle, sondern auch berufsrechtliche und weiterbildungsrechtliche Implikationen, und die ge-

990 DKI (Hrsg.), Krankenhaus Barometer, Umfrage 2007, S. 71 ff.

991 Von Rechenberg, Case-Manager: Wenn die Schwester den Arzt ersetzt (s. Literatur); Löcherbach, Ausbildung zur Case Managerin bzw. zum Case Manager in Deutschland (s. Literatur).

992 Siehe Amelung, DRG's und integrierte Versorgungssysteme: Neue Herausforderungen an das Personalmanagement von Krankenhäusern, in: Henke/Rich/Stolte (Hrsg.), Integrierte Versorgung und neue Vergütungsformen in Deutschland (2004), S. 214 ff.

993 Siehe zu den Grunddaten im Hinblick auf die Trägerschaft oben I.3.2.2.1 sowie insgesamt I.3.2. und I.3.3. 
deihliche Kostendeckung stellt sicherlich nicht das wirtschaftliche Optimum dar, dessen Erreichung den Anliegen des Sozialversicherungsrechts wiederum nicht notwendig entsprechen muß.

Das schließt nicht aus, die Anreizfunktion etwa des „Integrationsrechts“ durch Vertragsvolumina und Gestaltungsmodelle ebenso zu ermessen wie die Bedeutung juristischer Intervention bezogen auf wettbewerbsgefährdendes Verhalten, insbesondere in Form (nicht zwangsläufig) EG-vertragswidriger Verlustausgleiche für kommunale Häuser. Insgesamt jedenfalls zeigt sich, daß krankenhausindividuelle Marktstrategien und Managementkapazität weiter an Bedeutung zunehmen. Zugleich setzt erfolgsorientiertes Handeln voraus, normativ abgesteckte Spielräume zu erkennen und rechtskonform zu nutzen.

Entsprechende Spielräume räumt - dies immerhin könnten die Grunddaten ausweisen - offensichtlich das Privatrecht ein, sei es, daß Krankenhäuser als private Einrichtungen, sei es, daß öffentliche Einrichtungen in privatrechtlicher Form betrieben werden. Denn während die Zahl der stationären Einrichtungen insgesamt im ZehnJahreszeitraum von 1995-2005 von 2.325 ebenso wie die Zahl der öffentlichen (972) und der freigemeinnützigen Einrichtungen (944) deutlich und überwiegend kontinuierlich zurückging (2.139/751/818), ${ }^{994}$ nahm die Zahl der privaten Häuser von 409 auf 570 zu. Gleichzeitig stieg trotz Rückgangs der Zahl der öffentlichen Einrichtungen insgesamt die Zahl der in privatrechtlicher Form betriebenen öffentlichen Krankenhäuser, hinsichtlich derer seit dem Jahre 2002 nach ihrer Rechtsform differenziert wird, von 231 auf 332. Dennoch ist die Frage, „Macht die Trägerschaft einen Unterschied?“', keinesfalls vollständig geklärt oder einheitlich zu beantworten. 995 Gerade mit Blick auf Regelungen zu Mindestmengen aber dürfte zunehmend wichtiger werden, daß diese „im Kontext von Klinikketten leichter zu erreichen" sind. 996

Fest steht jedenfalls, daß die Trägerschaft als „Strukturmerkmal“ und diejenige als Rechtsform in enger Verbindung zueinander stehen, daß die Formenwahl ,unmittelbare betriebswirtschaftliche Konsequenzen zur Folge" hat und daß vor diesem Hintergrund zu konstatieren ist:

„Krankenhäuser in der Rechtsform privater Betriebe nehmen deutlich zu (insbesondere bei der

Rechtsform der $\mathrm{GmbH}$ ), wohingegen Häuser in der Rechtsform öffentlicher Betriebe abnehmen (hier insbesondere bei den Eigen- und Regiebetrieben).“997

Unter Effizienzgesichtspunkten schließlich dürfte zu würdigen sein, daß im besagten Zehn-Jahres-Vergleich einem Rückgang der Betten um ca. 80.000 auf rund 524.000 ein

994 StBA, Grunddaten der Krankenhäuser 2005 (2006), Allgemeiner Überblick 2005, sowie Ziff. I. 1.4.

995 Vgl. das Gutachten (2007) des Sachverständigenrates zur Begutachtung der Entwicklung im Gesundheitswesen, S. $360 \mathrm{ff}$.

996 A.a.O., S. 363.

997 DKI (Hrsg.), Krankenhaus Barometer, Umfrage 2007, S. 32 ff. 
Anstieg der Fallzahlen um mehr als 900.000 bei gleichzeitigem Rückgang der Verweildauer von 11,4 auf 8,6 Tage gegenüber steht. 998

\section{Wettbewerb zwischen den Versicherungsträgern}

\subsection{Gesetzliche Krankenkassen als Konkurrenten im Wettbewerb}

Das wettbewerbsorientierte Verhältnis der Krankenkassen zueinander manifestiert sich auch nach der Neuordnung der Finanzierungsströme vor allem in zwei Tätigkeitsfeldern, nämlich im Bereich des „Mitgliederwettbewerbs“ auf dem (höchst unvollkommenen) „Versicherungsmarkt" sowie bei der Nachfragetätigkeit in Ansehung spezifischer Versorgungsangebote und Kooperationen. Beide Felder sind - aus der Perspektive des Mitgliederwettbewerbs und ungeachtet des sozialrechtlichen Dreiecksverhältnisses - eng miteinander verbunden. Ähnliches gilt für das durch Werbung erfasste multipolare Beziehungsgeflecht, will man Werbung nicht überhaupt eigenständig betrachten.

Im direkten Wettbewerbsverhältnis der Krankenkassen zueinander geht es v.a. um einen Vergleich der wirtschaftlichen Leistungsfähigkeit nach Maßgabe und im Spiegel der Finanzierungsgrundlagen. Soweit das Wettbewerbsverhältnis im übrigen bereits durch die Wahl der Versicherung durch den Verbraucher 999 und die Bindung an ebenso leistungsfähige wie kostengünstige Krankenhäuser mittelbar geprägt ist, ${ }^{1000}$ war davon bereits die Rede.

Mit Blick auf die Krankenhausbehandlung rückt nunmehr ein Vergleich der Kostenbelastung bzw. der verfügbaren Mittel der Krankenkassen in den Vordergrund; dabei treten die Krankenhäuser zwar in Vorleistung, jedoch sind die Entgelte zeitnah an die Krankenhäuser und ggf. auch in Teilzahlungen zu entrichten, und bei Verzug bzw. verspäteter Zahlung drohen wirtschaftliche Folgen. ${ }^{1001}$ Entsprechend hohe Zahlungen belasten die Krankenkassen auch, wenn es - äußerstenfalls - darum geht, wie es um deren Zahlungs(un)fähigkeit 1002 bestellt ist, die im Rahmen einer nach insolvenzrechtlichen Grundsätzen zu erstellenden dynamischen Liquiditätsbilanz ${ }^{1003}$ zu ermitteln ist. Ungeachtet des (geringen Einflusses) selektiver Vergütungsunterschiede ist zu bedenken, daß die Leistungsausgaben für Krankenhausbehandlung entsprechend der Morbidität der Versicherten kassenindividuell deutlich differieren. Differenziert nach Kassenarten etwa fielen im 1.-4. Q 2008 bei den Ortskrankenkassen mit rund $€ 908$ je Versicherten für die Krankenhausbehandlung insgesamt wesentlich höhere Kosten an als bei den Innungs-

998 A.a.O., Ziff. I. 1.1.

999 Vgl. oben 2.

1000 S. 3.

1001 Vgl. § 11 Abs. 2 S. 3 KHEntgG.

1002 Vgl. § 171b Abs. 1 S. 2 SGB V i.V.m. § 17 InsO.

1003 S. Reischl, Insolvenzrecht, 2008, § 3 Rn. 102. 
krankenkassen (€ 560). ${ }^{1004}$ Damit rückt auch der Risikostrukturausgleich (RSA) in den Blickpunkt.

Maßgeblich für die Steuerung des Konkurrenzverhältnisses der gesetzlichen Krankenkassen untereinander sind danach v.a. zwei Regelungskreise: ein aus Sicht der solidarischen Wettbewerbsordnung GKV „interner“", in SGB V und RSAV verankerter Steuerungsmechanismus, sowie ein „externer“. Der erste betrifft neben Optionen verbleibender kassenindividueller Finanzierung den finanzwirksamen Ausgleichs- bzw. Verteilungsmechanismus, mit dem GKV-spezifische Wettbewerbsgrundlagen geschaffen werden, damit Wettbewerb nicht zu Lasten der allgemein kostenträchtigen Versorgung Kranker erfolgt, insbesondere also solidaritätswidrige Risikoselektion vermieden wird. Letzterer betrifft die Problematik, inwiefern Wettbewerbs- und Kartellrecht auf das Verhalten der Krankenkassen im Rahmen der diesen verbleibenden Spielräume kontrollierend einwirken oder aber Privilegierungen bzw. Bereichsausnahmen für diesen Sektor bestehen.

\subsubsection{Exkurs: Der Risikostrukturausgleich (RSA) alter Prägung}

Der interne Finanzierungsmechanismus fungiert als Wettbewerbsgrundlage im solidarischen System der GKV. Insoweit ist für die Idee des Wettbewerbs zwischen gesetzlichen Krankenkassen ein kurzer Abriss der Einführung des RSA alter Prägung unerlässlich.

Als echter Finanzausgleich stand der RSA alter Prägung unmittelbar im Zusammenhang mit der Einführung der Kassenwahlrechte der Versicherten. Das kassenartübergreifende, 1005 bundesweit angelegte System diente dem Ausgleich ausgewählter wettbewerbsrelevanter Strukturunterschiede zwischen den Krankenkassen:1006 Es waren die Einnahmenunterschiede aufgrund der unterschiedlichen Höhe der beitragspflichtigen Einnahmen der Mitglieder einer Krankenkasse (Grundlohnsummen) und Belastungsunterschiede aufgrund unterschiedlicher Anteile von beitragsfrei versicherten Familienangehörigen sowie v.a. wegen der unterschiedlichen Verteilung der Risiken in typisiert abzubildender Risikostruktur ( $§ 266$ Abs. 1 S. 2 und 3 SGB V aF). Um die Morbiditätsrisiken zu erfassen, wurden Alter, Geschlecht, der Bezug einer Erwerbsminderungsrente sowie die Einschreibung in DMP-Programme herangezogen (§ 266 Abs. 1 S. 2 und 3, $\S 267$ Abs. 2 SGB V a.F.). Andere Faktoren waren nicht ausgleichsfähig (§ 266 Abs. 1 S. 3 SGB V a.F.). Überstieg der Beitragsbedarf 1007 einer Krankenkasse deren Finanz-

1004 Mitglieder und Rentner zusammen; vgl. die vorläufigen Rechnungsergebnisse der gesetzlichen Krankenversicherung nach der KV 45 (BMG, 5.3.2009), S. 39. Bei den Ersatzkassen für Arbeiter $€ 580$, bei den Betriebskrankenkassen $€$ 605, bei den Ersatzkassen für Angestellte $€ 696$ und bei der KBS $€ 1.210$.

1005 Die landwirtschaftlichen Krankenkassen hingegen sind nicht beteiligt.

1006 Auf Details kann hier nicht näher eingegangen werden; vgl. diesbezüglich die Darstellung durch das $B V A$, Wie funktioniert der Risikostrukturausgleich? (Literatur).

1007 Vgl. § 266 Abs. 2 S. 2 SGB a.F.: „Der Beitragsbedarf einer Krankasse ist die Summe ihrer standardisierten Leistungsausgaben.“. 
kraft, 1008 stand dieser „Empfängerkasse“ ein Ausgleichsanspruch in Höhe der Differenz zu; im umgekehrten Fall bestand eine Zahlungsverpflichtung. 1009 Das BVA führt(e) den Ausgleich durch, $1010 \S 266$ Abs. 5 SGB V a.F., die zahlungstechnische Abwicklung oblag der DRV Bund (vgl. zum monatlichen Ausgleich bzw. der Selbstbescheidung der Krankenkassen sowie zum Zwischen- und Jahresausgleich v.a. §§ 17 und 19 RSAV).

Um Wettbewerbsnachteile der Krankenkassen mit ungünstigen Versichertenstrukturen abzubauen und Anreize für eine höhere Wirtschaftlichkeit im Gesamtsolidarsystem zu schaffen, wurden (und werden) nur die durchschnittlichen, standardisierten (und nicht die tatsächlichen) Leistungsausgaben berücksichtigt, vgl. vormals $§ 266$ Abs. $2 \mathrm{~S}$. 2 und 3 SGB V a.F. Aufwendungen für die Durchführung von DMP sind in die Ermittlung der durchschnittlichen Leistungsausgaben einzubeziehen, § 266 Abs. 4 S. 2 SGB V. Bis zum Inkrafttreten des weiterentwickelten RSA im Gesundheitsfonds existierte darüber hinaus ein Risikopool, mit dem aufwändige Leistungsfälle solidarisch finanziert wurden, § 269 (Abs. 8) SGB V.

Die Bedeutung des RSA für die wettbewerbsorientierte Organisationsreform der GKV wurde im Jahre 1993 in der Begründung zum GSG wie folgt beschrieben:

„Der ab 1. Januar 1997 mögliche Kassenwechsel kann... erstmals zwei Jahre nach der vollständigen Einführung des Risikostrukturausgleichs zwischen allen Krankenkassen... ausgeübt werden. Dieser Übergangszeitraum ist unabdingbar, um größtmögliche Chancengleichheit zwischen allen Krankenkassen herzustellen. Erst nach Durchführung des kassenartübergreifenden Risikostrukturausgleichs sind gleiche Wettbewerbsbedingungen für alle Krankenkassen vorhanden.“1011

5.1.2. Interner Steuerungsmechanismus: Neuordnung der Finanzierungsströme im Zeichen des Gesundheitsfonds 1012

\subsubsection{1. (Risikoadjustierte) Zuweisungen aus dem Gesundheitsfonds}

Seit dem Ausgleichsjahr 2009 ist der RSA integraler Bestandteil des Gesundheitsfonds. ${ }^{1013}$ Aus diesem erhalten die Krankenkassen Zuweisungen, bestehend aus einer Grundpauschale sowie alters-, geschlechts- und risikoadjustierten $\mathrm{Zu}$ - und Abschlägen

$1008 \S 266$ Abs. 3 S. 1 SGB V a.F.: „Die Finanzkraft einer Krankenkasse ist das Produkt aus den beitragspflichtigen Einnahmen ihrer Mitglieder und dem Ausgleichsbedarfssatz".

1009 S. zur Höhe der Ausgleichsforderungen und -verpflichtungen im RSA 2006 bei 182 Krankenkassen mit Zahlungsverpflichtungen in Höhe von insgesamt $€$ 13,9 Mrd. und 59 Empfängerkassen mit Ansprüchen in Höhe von $€ 16.3$ Mrd. Otto/Göpffarth, in: Göpffarth, u.a., Jahrbuch Risikostrukturausgleich (2008), S. 301 (319).

1010 Bzw. führt ihn durch, soweit das Ausgleichsjahr mit dem Jahresausgleich (im Herbst des Folgejahres) noch nicht abgeschlossen ist.

1011 BT-Drucks. 12/3608, S. 74.

1012 S. Pfohl, Die Neuregelung der Finanzierungsströme durch den Gesundheitsfonds, in. Göpffarth, u.a. (Hrsg.) Jahrbuch Risikostrukturausgleich 2007 (2007), S. $195 \mathrm{ff}$.

1013 Pfohl/Sichert, Der Gesundheitsfonds: Sondervermögen des Bundes oder der Krankenkassen?, NZS 2009, S. 71 (72 f.). 
(vgl. § 266 Abs. 1 S. 1 SGB V) und Zuweisungen für sonstige Ausgaben (§ 270 SGB V). ${ }^{1014}$ Soweit deren besondere Voraussetzungen erfüllt sind, erfolgt im Zeichen der neu gefassten Konvergenzklausel (§ 272 SGB V) gem. dessen Abs. 2 S. $1^{1015}$ eine Erhöhung der Zuweisungen.

Für die Durchführung ergibt sich aus $\S 39$ Abs. 2 RSAV die verfahrensrechtliche Differenzierung zwischen der grundlegenden Feststellung der kassenindividuellen Werte (vorläufige Höhe der Zuweisungen gem. S. 1, „Grundlagenbescheid“ 1016) und der darauf basierenden Zuweisung der Mittel in Abhängigkeit der Versichertenzahl (S. 2, Zuweisungsbescheid). Nach $\S 39$ Abs. 3 S. 1 RSAV berechnet das BVA alsdann für alle Krankenkassen im Rahmen mehrerer Strukturanpassungen 1017 die vorläufige Höhe der Zuweisungen unter Berücksichtigung der Datenmeldung nach $\S 32$ RSAV (aktuelle Daten über die Versichertenstruktur) neu (neue Zuweisungsgrundlage); die bis dahin geleisteten Zuweisungen für das Ausgleichsjahr werden auf dieser Grundlage neu ermittelt ( $\$ 39$ Abs. 3 S. 2 RSAV) und korrigiert ( 39 Abs. 3a RSAV, Korrekturbescheid). Der Jahresausgleich erfolgt nach $\S 41$ RSAV.

Wesentlich für die risikoadjustierten Zuschläge entsprechend der morbiditätsgruppenorientierten Versichertenklassifikation nach Maßgabe der (derzeit) 80 ausgewählten Krankheiten 1018 sind die diagnosebezogenen Datenmeldungen der Krankenkassen. ${ }^{1019}$ Betreffend die Aufgreifkriterien für einen Morbiditätszuschlag sind Krankenhausdiagnosen 1020 von besonderer Wertigkeit: Jede (krankheits-)relevante stationäre Diagnose bedingt eine Zuordnung der Versicherten zu einer Morbiditätsgruppe und hat somit einen Zuschlag zur Folge. ${ }^{1021}$

Bereits aus der Datenbasis für die Weiterentwicklung sind unterschiedliche Belastungen bzw. Risikostrukturen der Kassen erkennbar. Bezogen zunächst auf die Stichprobe zur Weiterentwicklung des RSA - für die nachfolgende Ermittlung der vorläufigen Werte zur Durchführung war dann die Vollerhebung maßgeblich - waren bspw. $21,2 \%$ der seitens der KBS gemeldeten Versichertenpseudonyme solche mit Krankenhausaufenthalten; die Quote bei der AOK betrug 15,16\%, die der IKK 10,88\%. ${ }^{1022} \mathrm{Da}$ -

1014 Zur Anpassung der Zuweisung in der Konvergenzphase § 272 SGB V, §§ 33a ff. RSAV, jeweils idF. des GKV-OrgWG; die zur Erhöhung der Zuweisungen erforderlichen Beträge werden aus Mitteln der Liquiditätsreserve (§ 271 Abs. 2 SGB V) aufgebracht, § 272 Abs. 2 S. 2 SGB V.

1015 I.V.m. §§ 33a ff. RSAV, vgl. insbes. § 33c Abs. 2 RSAV.

1016 Siehe BT-Drs. 16/10609 (elektron. Vorabfassung), S. 90.

1017 Zum 31. März und zum 30. September des Ausgleichsjahres sowie zum 31. März des auf das Ausgleichsjahr folgenden Jahres.

$1018 \S \S 268$ Abs. 1 S. 1 SGB V, $\S 29$ Nr. 1 RSAV sowie $\S 31$ RSAV. Die Festlegungen trifft das BVA nach $\S 31$ Abs. 4.

1019 Vgl. § 30 RSAV.

1020 S. $\S 30$ Abs. 1 s. 1 Nr. 6 RSAV i.V.m. Meldevorschriften des SGB V.

1021 Göpffarth, Wie der morbiditätsorientierte Risikostrukturausgleich funktioniert, SozSich 2009, S. 12 (13 f.).

1022 Auf Grundlage der Darstellung bei Schmidt/Wittmann/Göpffarth, in: Göpffarth u.a., Jahrbuch Risikostrukturausgleich (2008), S. 275 (289, 291). 
bei entfielen bei der KBS auf einen Versicherten mit Krankenhausaufenthalt im Schnitt 10,5 Diagnosen, wohingegen bei der AOK 8,3 und der IKK 6,8 zu verzeichnen waren. 1023

Auch im Zusammenhang mit dem RSA sind Versuche der Einflussnahme auf das ärztliche Kodierverhalten betreffend (RSA-) relevante ambulante Diagnosen bekannt geworden. ${ }^{1024} \mathrm{Im}$ RSA setzt die Zuordnung zu Morbiditätsgruppen mittels ambulanter Diagnosen eine Zweitdiagnose in einem weiteren Quartal (,M2Q“) und ggf. zusätzliche Validierung durch Arzneimitteltherapie voraus. Das Bestreben, Daten nachzuerfassen oder zu korrigieren, versuchten einige Krankenkassen damit zu rechtfertigen, sie betrieben keine Manipulation bzw. „up-“, sondern „right-coding“. Dieser lediglich phänomenologische Terminus „right-coding“ indes wird uneinheitlich verwendet und leitet fehl, reklamiert er doch sachverhaltsunspezifisch und gleichsam begriffsjuristisch eine antizipierte Rechtfertigung. ${ }^{1025}$ Demgegenüber sind Einflussnahmen in jedem Einzelfall zu würdigen und als solche in aller Regel unzulässig.

Der M-RSA im Gesundheitsfonds 1026 bildet keine „Ist-Morbidität“ ab, sondern die für die risikoadjustierten Zuweisungen maßgebliche morbiditätsorientierte Zuordnung folgt daraus, dass Versicherte aufgrund ihrer Morbidität im maßgeblichen Zeitpunkt unter entsprechender Diagnosestellung behandelt wurden und deswegen Leistungsausgaben verursacht haben. Der RSAV knüpft, vgl. § $30 \mathrm{RSAV}$, „nur“ an - vorliegende (Abrechnungs-)Daten an, die Ergebnis der Erbringung von Versicherungsleistungen zu einem bestimmten Zeitpunkt (Quartal) aus konkretem Behandlungsanlass sind, soweit die Behandlung nach Auffassung des Arztes indiziert war; § 295 Abs. 1 S. 2 SGB V (Verschlüsselung nach den Vorgaben des Deutschen Instituts für medizinische Dokumentation und Information (DIMDI)) ist zu beachten. Durch „Zuweisungsrelevanz“ im RSA motivierte Nacherhebungen oder Korrekturen sind melde-, berufs- und datenschutzrechtlich (vgl. § 284 SGB V) unzulässig. ${ }^{1027}$ Hier ist aufsichtsrechtliche Steuerung ebenso angezeigt, wie gesetzgeberisches „Nachsteuern“1028, das durch Schaffung des neuen $§ 273$ SGB V, einer erweiterten Plausibilitätsprüfung ex ante, erfolgte. 1029

1023 A.a.O., S. 291.

1024 Ausf. Göpffarth/Sichert, Morbi-RSA und Einflussnahmen auf ärztliches Kodierverhalten, KrV 2009, S. $186 \mathrm{ff}$.

1025 Göpffarth/Sichert, a.a.O., S. 187 f.

1026 S. dazu unter II.5.1.2.

1027 Göpffarth/Sichert, Morbi-RSA und Einflussnahmen auf ärztliches Kodierverhalten, KrV 2009, S. 186 (188 ff.).

1028 S. oben I.2.3.1.8.

1029 Näher Göpffarth/Sichert, Morbi-RSA und Einflussnahmen auf ärztliches Kodierverhalten, KrV 2009, S. 186 (190). 


\subsubsection{Kassenindividuelle Finanzierungsinstrumente (Zusatzbeiträge, Prämien)}

Vergleichbar dem vormals kassenindividuell erhobenen Beitragssatz kommt den neuen zusätzlichen Wettbewerbsinstrumenten ${ }^{1030}$ Zusatzbeitrag (§ 242 Abs. 1 SGB V) und Prämie (Abs. 2) besondere Publizitätsfunktion zu. Die tarifäre Vergleichbarkeit der allgemeinen Versicherungsleistung wird für den zu Versichernden dadurch offenbar, daß Kassen am Markt auftreten, die einen Zusatzbeitrag erheben, daß andere ohne einen solchen auskommen und wiederum andere gar eine Prämie ausschütten.

Um Details der Ausgestaltung von Zusatzbeitrag und Prämie ranken sich jenseits der zulässigen Erhebung bzw. Ausschüttung als Pauschalbetrag bzw. in Prozent der beitragspflichtigen Einnahmen 1031 zahllose Einzelfragen, die hier nicht weiter verfolgt werden können, 1032 zumal sie nicht in besonderem Zusammenhang mit Steuerungsfragen bei der Krankenhausversorgung stehen. Unter Steuerungsgesichtpunkten gleichwohl interessant sind drei Aspekte:

Der erste betrifft das strategische Verhalten unter dem Eindruck der „Besorgnis“, (als erster) einen Zusatzbeitrag erheben zu müssen, was bis in das zweite Quartal des Jahres 2009 hinein nicht der Fall war. Jede gesetz- oder verordnungsgeberische Änderung im Anschluss an das GKV-WSG wurde zudem besonders kritisch dahingehend beäugt, ob sie als ,zusatzbeitragsrelevant“" erschien. Nicht zuletzt deshalb auch bestand das Bestreben, das Zuweisungsverfahren und Korrekturen stets möglichst nah an das im Jahresausgleich zu erwartete Ergebnis heranreichen zu lassen. Dennoch führen u.U. auch die Strukturanpassungen, d.h. die Neuberechnungen der vorläufigen Höhe der Zuweisungen mit entsprechenden Korrekturbescheiden (vgl. § 39 Abs. 3, 3a RSAV) einer Krankenkasse vor Augen, inwieweit es mit Blick auf die Vorgaben des $\S 242$ Abs. 1 SGB V geboten ist, einen Zusatzbeitrag zu erheben. Gleichzeitig hatte noch vor der Jahreswende 2008/2009 eine massive Fusionswelle eingesetzt, um Zusatzbeiträgen, zumal als kleine Kasse, zu entkommen.

Der zweite Aspekt betrifft den Fall, daß es tatsächlich zur Erhebung eines Zusatzbeitrages kommt. Der komplexe Prozess der Erhebung und Berechnung v.a. dann, wenn der Zusatzbeitrag nicht als Pauschale erhoben werden sollte, hat zu intensiven Überlegungen zum „Outsourcing“ eines Zusatzbeitragsmanagements geführt. Das auch im Bereich der Selbstverwaltung nicht per se unzulässige „contracting out“ bewegt sich zwischen funktionaler (Erfüllungs-) Privatisierung sowie einer Finanzierungs- und Verfahrensprivatisierung, muss allerdings die Erhebung in Form der Festsetzung als Kernaufgabe der Kasse ebenso belassen, wie die Verantwortlichkeiten klar zuweisen, den Sozialdatenschutz beachten und schließlich von gewerblichen Koppelungsgeschäften Abstand nehmen.

1030 BT-Drs. 16/3100, S. 165.

1031 A.a.O.

1032 Dazu etwa Wolf, Der neue Zusatzbeitrag nach § 242 SGB V, GesR 2008, S. 567 ff. 
Drittens wird es - im gegebenen System - u.U. zu massiven Problemen führen, daß die Erhebung des Zusatzbeitrag auf 1 vom Hundert der beitragspflichtigen Einnahmen begrenzt ist ( $\$ 242$ Abs. 1 S. 2 SGB V). Dies gilt v.a. mit Blick auf eine mögliche ,geplante Unterdeckung“" des Gesundheitsfonds (vgl. § 220 Abs. 2 S. 1 SGB V) oder bezogen auf zusätzliche Finanzierungslasten etwa aus Haftungsverbünden bei Schließung bzw. Insolvenz. Als echtes bzw. zusätzliches „Wettbewerbsinstrument“ kann der Zusatzbeitrag dann letztlich nicht mehr bezeichnet werden.

\subsubsection{Externer Kontrollmechanismus: Zur Anwendung des Wettbewerbs- und Kartellrechts}

Der Stabilisierung des Fundamentes für den Kassenwettbewerb dient neben den systeminternen Finanzierungs- und Ausgleichsmechanismen ein externes Kontrollsystem zur Unterbindung wettbewerbswidrigen bzw. marktmissbräuchliches Verhaltens. Die Bestimmung und die Konsequenzen des materiellen Kontrollrahmens allerdings werden kontrovers diskutiert.

\subsubsection{Allgemeines und Mitgliederwerbung}

Keinem Zweifel unterliegt, daß für den Wettbewerb der Krankenkassen untereinander betreffend die Mitgliederwerbung gem. $§ 51$ Abs. 1 Nr. 2, Abs. 2 S. 1 SGG grundsätzlich der Rechtsweg zu den Sozialgerichten eröffnet ist und das Rechtsverhältnis diesbezüglich v.a. durch die sozialrechtlichen Vorschriften ( $§ 207$ ff., 265 ff. SGB V, $\S \S 13$ ff. SGB I u.a.) bestimmt ist. ${ }^{1033}$ Bereits aus $\S 51$ Abs. 2 S. 2 SGG folgt überdies, daß der Rechtsweg auch bei privatrechtlichen Streitigkeiten in Angelegenheiten der GKV gegeben ist, d.h. auch in wettbewerbsrelevanten Zusammenhängen. 1034

Eine auch in diesem Zusammenhang strittige Frage aber ist, ob z.B. mit Blick auf wettbewerbswidrige Werbung auch die Anwendung des materiellen Lauterkeitsrechts ausgeschlossen ist, und falls nein, ob auch dann ausschließlich der Weg zu den Sozialgerichten eröffnet ist. Die Problematik betrifft das Verhältnis des $\S 69$ SGB V zur Rechtswegzuweisung und die Reichweite der Norm zugunsten sämtlicher Konstellationen mit sozialrechtlichem Bezug bzw. die Frage der Regelung der Rechtsbeziehung zwischen den Krankenkassen ausschließlich durch öffentliches Recht. ${ }^{1035}$ So wird vertreten, das Verhalten der Krankenkassen im Mitgliederwettbewerb betreffe stets das öffentlich-rechtlich geregelte - Verhältnis zu den Versicherten und deshalb gelte ausschließlich Sozialrecht. ${ }^{1036}$ Indes lassen sich Fragen des Rechtsweges und des Anwendungsausschlusses materiellen Rechts durch $\S 69$ SGB V durchaus voneinander unter-

1033 BGH, Urt. v. 9.11.2006, GRUR 2007, S. 535 (536).

1034 Vgl. Groß, in: Hk-SGG, § 51 Rn. 19.

1035 Vgl. BSGE 82, 87.

1036 Vgl. Roth, Kartellrechtliche Aspekte der Gesundheitsreform nach deutschem und europäischem Recht, GRUR 2007, S. 645 (648 f.). 
scheiden. Die (mögliche) Trennung von Rechtsweg und Kontrollmaßstab kommt in $\S$ 51 Abs. 2 S. 1 SGG zum Ausdruck; sie soll allerdings nach strittiger ${ }^{1037}$ Auffassung nicht nur für die kartellrechtliche Würdigung der Mitgliederwerbung gesetzlicher Krankenkassen im Verhältnis zu PKV-Unternehmen, ${ }^{1038}$ sondern auch für einen privatrechtlichen Kontrollmaßstab des Kassenwettbewerbs gelten. 1039

Gleichwohl kann zunächst nur gesagt werden, daß sich die materielle Exklusivität des Sozialrechts jedenfalls auf $\S 69$ SGB V nicht eindeutig stützten lässt. ${ }^{1040}$ Die Vorschrift betrifft nicht das Verhältnis der Krankenkassen zueinander, ${ }^{1041}$ sondern nur das der Krankenkassen und ihrer Verbände zu den Leistungserbringern, wenngleich auch, soweit Dritte betroffen sind (etwa bei Konkurrenz mit privilegierten Leistungserbringern wegen deren „Beteiligung“ an der Erfüllung von Versorgungsaufgaben im Selektivvertragsverbund mit Kassen). Zur Erweiterung als „Grundnorm“ ausschließlich öffentlich-rechtlichen Handelns 1042 reicht die Fassung rechtssystematisch nicht aus. ${ }^{1043}$ Da $\S 69$ Abs. 1 S. 1 bis 3 SGB V auch im Verhältnis zu „Dritten“ nur gilt, sofern deren Rechte durch „diese Rechtsbeziehungen... betroffen“ sind, § 69 Abs. 1 S. 4 SGB V, scheidet eine auch aus systematischen Gründen ${ }^{1044}$ verfehlte Qualifikation anderer gesetzlicher Krankenkassen als „Dritten“ aus.

Dessen ungeachtet ist zu konstatieren, daß die eigentlichen Maßstäbe und Grenzen der Werbung ganz überwiegend durch das öffentliche Recht vorgegeben werden. Deutlich macht dies die im Zivil-1045 ebenso wie im Sozialrechtsweg 1046 behandelte The-

1037 Siehe BGH, Beschl. v. 9.11.2006, I ZB 23/06, GRUR 2007, S. 535 (536 m. zahlr. N.). Zur Kritik an der Entscheidung des BGH bezüglich dessen Zuständigkeit auch Roth, a.a.O. (S. 648): „Freilich ist dieser Lösungsansatz keineswegs über alle Zweifel erhaben. Denn auch im Bereich der Mitgliederwerbung handeln die gesetzlichen Krassen ebenfalls mit Bezug auf ihren gesetzlichen Versorgungsauftrag“".

1038 Vgl. auch Roth, a.a.O., S. 649.

1039 Vgl. auch Steinmeyer, Wettbewerbsrecht im Gesundheitswesen (2000), S. 82 ff.

1040 Vgl. aber Knispel, Doppelter Rechtsweg für Klagen gegen Mitgliederwerbung einer Krankenkasse?, NZS 2008, S. 129, und Keller, in: Meyer-Ladewig/Keller, Leitherer, SGG, 9. Aufl. (2008), $\S 51$ Rn. 23.

1041 Engelmann (2009), jurisPK SGB V, § 69 Rn. 144.

1042 Siehe die Nachweise bei Mühlhausen, Mitgliederwettbewerb innerhalb der gesetzlichen Krankenversicherung (2002), S. 58 Fn. 19, S. 58 f.

1043 Mühlhausen, a.a.O., S. 58 f. m.w.N. Der Rekurs auf die Begründung, die Kassen handelten „nicht als Unternehmen im Sinne des Privatrechts, einschließlich des Wettbewerbs- und Kartellrechts“ stellt eine inhaltliche Abkopplung vom ersten Satzteil dar: „Die Krankenkassen und ihre Verbände erfüllen in diesen Rechtsbeziehungen ihren öffentlich-rechtlichen Versorgungsauftrag“".

1044 Mühlhausen, a.a.O., S. 57.

1045 S. LG Itzehoe, Urt. v. 10.06.2008, 5 O 32/07, 6 U, 28/08, WRP 2009, S. 494: Irreführende Werbung i.S.d. $\S \S 3$ und 5 UWG: „Wirbt eine Krankenkasse allein mit dem Hinweis auf die ab dem 1.1.2009 geltenden einheitlichen Beitragssätze, ist dies irreführend, weil der unzutreffende Eindruck erweckt wird, die finanziellen Belastungen der Versicherten seien bei allen Kassen im Ergebnis gleich."

1046 S. mit Blick auf $\S \S 13-15$ SGB I, § 86 SGB X und $\S 4$ Abs. 3 SGB V LSG Rheinland-Pfalz, Beschl. v. 13.12.2007, L 5 ER 289/07 KR: „Die Krankenkasse darf nicht mit der Aussage werben, ... ohne 
matik der Werbung unter Verweis auf den allgemeinen und gleichen Beitragssatz ab 2009 ohne Information über die Möglichkeit kassenindividueller Zusatzbeiträge oder Prämien. Ob der Sanktionsanspruch hier zivilrechtlicher (vgl. § 8 UWG) oder öffentlich-rechtlicher Natur ist (öffentlich-rechtlicher Unterlassungsanspruch entsprechend § 1004 BGB bzw. grundrechtlich fundiert), kann nicht entscheidend sein. In der Sache handelt es sich jedenfalls um Angelegenheiten der Krankenversicherung, die im Sozialrechtsweg zu verfolgen sind. ${ }^{1047}$ Das gilt mit Blick auf $\S 51$ Abs. 2 S. 1 SGG auch für eine Streitigkeit zwischen privaten und gesetzlichen Krankenversicherungen. 1048

Eindeutiger sind die Fälle, in denen Fragen des Mitgliederwettbewerbs im Verhältnis der Krankenkassen zu den Leistungserbringern wurzeln, etwa wenn die Krankenkasse im Mitgliederwerb Werbung für günstige Leistungserbringer macht, mit denen sie Vertragsbeziehungen eingegangen ist. 1049 Diese Vorgehensweise kommt v.a. in Betracht, wenn die Kasse mit Vertragsbeziehungen zu Krankenhäusern in IV-Modellen und DMP wirbt. Ist dies aber der Fall, kann es, nimmt man die materielle Ausschlusswirkung des $\S 69$ SGB V ernst, die auch der $B G H$ anerkannt hat, nicht darauf ankommen, ob der Anspruch ,nicht auf einen Verstoß gegen Vorschriften des SGB V, sondern ausschließlich auf wettbewerbsrechtliche Normen gestützt [wird], deren Beachtung auch jedem privaten Mitbewerber obliegt“". ${ }^{1050} \mathrm{Im}$ Falle erheblicher Beeinträchtigung einzelner Leistungsanbieter - etwa durch grob irreführende oder sozialrechtliche Vorschriften verletzende Werbung - wäre ggf. auf eine Beeinträchtigung der Berufsfreiheit drittbetroffener (privater) Grundrechtsträger zu rekurrieren. ${ }^{1051}$ Darauf (i.V.m. mit der Verletzung sozialrechtlicher Grenzen) kann ggf. auch ein Unterlassungsanspruch gestützt werden. 1052

\subsubsection{Fusionskontrolle}

Auch die Fusion gesetzlicher Krankenkassen unterliegt staatlicher Kontrolle. Strittig allerdings ist, ob die kartellrechtlichen Maßstäbe einer Fusionskontrolle (§§ 35 ff. GWB) zur Anwendung kommen. ${ }^{1053}$ Immerhin hatten Konzentrationsprozesse zur Fol-

auf die Möglichkeit der Erhebung eines kassenindividuellen Zusatzbeitrages und der Auszahlung von Prämien an Mitglieder... hinzuweisen.“ Vgl. dagegen LSG Baden-Württemberg, Beschl. v. 26.8.2008 am Maßstab des $\S 175$ Abs. 2 und 4 SGB V: „Grenzen des Wettbewerbs zwischen den Krankenkassen nicht überschritten.“

1047 Knispel, Doppelter Rechtsweg für Klagen gegen Mitgliederwerbung einer Krankenkasse?, NZS 2008, S. 129, und Keller, in: Meyer-Ladewig/Keller, Leitherer, SGG, 9. Aufl. (2008), § 51 Rn. 23.

1048 Knispel, a.a.O.; Keller, a.a.O., jeweils entgegen BGH, Beschl. v. 9.11.2006, I ZB 23/06, GRUR 2007, S. 535

1049 SG Frankfurt/Main v. 9.8 2006, GewArch 2006, 487 ff.

1050 Vgl. allerdings BGH, Beschl. v. 9.11.2006, GRUR 2007, S. 535. Näher zum materiell-rechtlichen Kontrollmaßstab unten II.5.1.2.

1051 Vgl. BSGE 89, 24 (33 f.).

1052 SG Frankfurt/Main v. 9.8 2006, GewArch 2006, 487 (488).

1053 In diesem Sinne Lübbig/Klasse, Kartellrecht im Pharma- und Gesundheitssektor (2007), S. 46 f. 
ge, daß mitunter Mitgliederbestände von wenigen Tausenden auf mehrere Hunderttausend „angewachsen“1054 waren. Am Vorabend der Einführung des Gesundheitsfonds nahmen die Fusionen nochmals deutlich zu, wodurch Krankenkassen mit ganz erheblicher Marktmacht entstanden. ${ }^{1055} \mathrm{Im}$ Zuge des GKV-WSG wurde ferner die kassenartübergreifende Vereinigung der Kassen möglich, § 171a SGB V; ein entsprechender Beschluß der Verwaltungsräte bedarf der Genehmigung der vor der Vereinigung zuständigen Aufsichtsbehörden. ${ }^{1056} \S 171$ a SGB V selbst jedenfalls schließt die Anwendbarkeit der $\S \S 35$ ff. GWB nicht aus. Die Vorschrift wurde vor dem Hintergrund eingeführt, daß das BKartA bereits zuvor Kassenfusionen geprüft hatte, ${ }^{1057}$ worauf der Gesetzgeber in seiner Begründung selbst hingewiesen hatte. ${ }^{1058}$ Dieser Passus wird mitunter als Beleg für den gesetzgeberischen Willen zugunsten der Anwendbarkeit der Fusionskontrollvorschriften genommen, ${ }^{1059}$ während anderer Auffassung zufolge die Streichung in der Beschlussfassung des Ausschusses und die Ablehung einer klarstellenden Regelung zugunsten der Anwendbarkeit der GWB-Vorschriften vom Gegenteil zeugen, ohne daß der de lege lata letztlich (doch) nicht verfügte Ausschluß interpretatorisch herbeizuführen wäre. ${ }^{1060}$

Das GKV-OrgWG hat die Frage der Anwendung kartellrechtlicher Kontrollmaßstäbe erneut offen gelassen. Ein Rückschluss aus der begrenzten Inbezugnahme im erweiterten $\S 69$ Abs. 2 SGB V überzeugt noch nicht, da auch dann, wenn der Zusammen-

1054 Vgl. etwa die Chronik der Taunus-BKK (unter www.taunus.bkk.de) und zur Fusionskontrolle durch das BKartA (unv.), Gaßner/Ahrens, Anwendbarkeit der Regeln der Fusionskontrolle des GWB bei der Vereinigung gesetzlicher Krankenkassen, SGb 2007, S. 528 (535).

1055 Davon zeugt etwa die Fusion von TK und IKK direkt.

1056 Siehe oben unter I. 4.5.3.

1057 Ungeachtet dessen wäre die Konturierung materiellrechtlicher Maßstäbe durch das BKartA interessant. Sie beträfe bereits die Betrachtung des sachlich relevanten Marktes, der als „Angebotsmarkt für Leistungen der gesetzlichen Krankenversicherung“ mit Blick auf die systemimmanenten Besonderheiten womöglich nur „unvollkommen“ beschrieben ist, Gaßner/Ahrens, Anwendbarkeit der Regeln der Fusionskontrolle des GWB bei der Vereinigung gesetzlicher Krankenkassen, SGb 2007, S. 528 (534). Die bisher bekannten (beiden) „Fälle“ der Fusionskontrolle durch das BKartA, in denen es nicht zur Untersagung des Zusammenschlußvorhabens kam, sind unveröffentlicht geblieben, a.a.O., S. 535.

1058 Siehe BT-Drucks. 16/3100, S. 156: „Flankierende gesetzliche Regelungen, die verhindern, daß durch kassenartübergreifende Fusionen wettbewerbsschädliche Monopolbildungen entstehen, sind nicht erforderlich. Auch Vereinigungen von Krankenkassen sind nach den Regeln der Fusionskontrolle des Gesetzes gegen Wettbewerbsbeschränkungen (GWB) durch das Bundeskartellamt zu prüfen. Das Bundeskartellamt hat bisher schon Vereinigungen von Krankenkassen daraufhin geprüft, ob sie zur Entstehung einer marktbeherrschenden Stellung führen. Auch kassenartübergreifende Fusionen wird es daraufhin überprüfen und bei Entstehung oder Verstärkung einer marktbeherrschenden Stellung untersagen.“.

1059 Gaßner/Ahrens, Anwendbarkeit der Regeln der Fusionskontrolle des GWB bei der Vereinigung gesetzlicher Krankenkassen, SGb 2007, S. 528 (532).

1060 Roth, Kartellrechtliche Aspekte der Gesundheitsreform nach deutschem und europäischem Recht, GRUR 2007, S. 645 (656). 
schluss primär ,auf der Nachfrageseite Sinn“ habe, 1061 im Grunde das Verhältnis der Krankenkassen zueinander betroffen ist. Dieses Verhältnis als solches wird von $§ 69$ SGB V nicht unmittelbar erfasst. 1062

Demgegenüber bringt $\S 69$ SGB V jedenfalls zum Ausdruck, daß die Krankenkassen nach Vorstellung des nationalen Gesetzgebers grundsätzlich nicht als Unternehmen tätig werden, bestenfalls ausgewählte Vorschriften des GWB „entsprechend“ zur Anwendung gelangen. (Auch) nach deutschem Kartellrecht lassen sich die Krankenkassen daher nicht als solche bzw. per se als Unternehmen qualifizieren. Und obwohl die Ergebnisse im Hinblick auf ein bestimmtes Tätigwerden in Anknüpfung an die Rspr. des FENIN-Urteils divergieren können, gelangt man zum selben Ergebnis, wenn man der Auffassung folgt, im Zuge der 7. GWB-Novelle sei eine übereinstimmende Auslegung der nationalen und europarechtlichen Kartellvorschriften im Hinblick auf den Unternehmensbegriff indiziert. 1063

Zudem beschreiben Fusionen keinen „bestimmten Markt“, auf dem Krankenkassen autonom wirtschaften, d.h. Martkanteile preisbewertet „kaufen“ könnten. Gestützt wird das Ergebnis durch die Spezialität der Vorschriften über die Vereinigung von Krankenkassen nach dem SGB V,1064 das insoweit auch Exklusivität reklamiert. Der Genehmigung durch die Aufsichtsbehörde kommt rechtsgestaltende Wirkung zu; überdies kann die Vereinigung ggf. durch Rechtsverordnung erfolgen (vgl. § 168a Abs. 2 SGB V). Weiter kann die durch GKV-OrgWG in ihren Befugnissen umfassend gestärkte Aufsichtsbehörde 1065 im Zusammenwirken mit dem Spitzenverband Bund den Zusammenschluss aktiv betreiben, vgl. § 172 Abs. 3 SGB V und ggf. ersetzende Beschlüsse fassen. Die insoweit nicht marktgeleitete Vereinigung der Krankenkassen als Körperschaften des öffentlichen Rechts und Teil der mittelbaren Staatsverwaltung kann damit der allgemeinen Fusionskontrolle für Unternehmen grundsätzlich nicht unterstellt werden, sondern vollzieht sich im Rahmen aufsichtsrechtlicher Genehmigung (vgl. § 144 Abs. 3, $\S 150$ Abs. 1 S. $2, \S 160$ Abs. 1 S. 2 , § 168 Abs. 1 S. 2 SGB V). ${ }^{1066}$

1061 Engelmann (2009), jurisPK-SGB V, § 69 Rn. 163.

1062 Vgl. in vorliegendem Zusammenhang Roth, Kartellrechtliche Aspekte der Gesundheitsreform nach deutschem und europäischem Recht, GRUR 2007, S. 645 (655); Gaßner/Ahrens, Anwendbarkeit der Regeln der Fusionskontrolle des GWB bei der Vereinigung gesetzlicher Krankenkassen, SGb 2007, S. $528 \mathrm{ff}$.

1063 Roth, a.a.O., S. 645, 656, 659, der gleichwohl eine unternehmerische Tätigkeit im Selektivvertragsbereich ablehnt.

1064 S. auch Eggert, Krankenkassen und Kartellamt: Fusionskontrolle zum Schutz des Wettbewerbs in der GKV?, VSSR 2007, S. $335 \mathrm{ff}$.

1065 Vgl. oben I.4.5.3.

1066 Zur Kontroverse über den Umfang aufsichtsrechtlicher Kontrollmöglichkeiten Mühlhausen, in: Becker/Kingreen: SGB V, 2008, § 144 Rn. 8 m.w.N. 


\subsection{4. „Ausschluss vom Markt“: Schließung und Insolvenz}

Im Zuge der Herstellung der Insolvenzfähigkeit aller Krankenkassen (vgl. § 171b SGB V m.W.v. 1.1.2010) droht diesen mit der Eröffnung des Insolvenzverfahrens bzw. der Ablehnung mangels Masse die Schließung, § 171b Abs. 5 SGB V. Es gelten die Insolvenzeröffnungsgründe des Insolvenzrechts, §§ 17-19 InsO. § 171b Abs. 2 SGB V ist einerseits Rechtsgrundverweisung; andererseits hat der Gesetzgeber ausweislich der Begründung zum GKV-OrgWG auch hinsichtlich der insolvenzrechtlichen Vorschriften Art. 74 Abs. 1 Nr. 12 (Sozialversicherung) als Kompetenztitel in Anspruch genommen und als „Sache der Sozialversicherung“ begriffen, ${ }^{1067}$ nicht jedoch Art. 74 Abs. 1 Nr. 1 GG herangezogen, der die Verlängerung der Befristung des zweistufigen Überschuldungsbegriffs (s.u.) trägt. Die sozialstaatliche Prägung kann damit bei der Auslegung nicht außer Betracht bleiben.

Gerade unter dem Eindruck hoher Verbindlichkeiten für Krankenhausrechnungen muss andererseits ein Liquiditätsdefizit der Kassen vermieden werden, damit es nicht zur Zahlungsunfähigkeit kommt (vgl. § 17 InsO). Maßgeblicher Beobachtungszeitraum für die insoweit vorzunehmende dynamische Liquiditätsbilanz ist - grundsätzlich - ein stichtagseröffneter Drei-Wochen-Zeitraum, durch den auch Zahlungsstockungen aus der Betrachtung auszunehmen sind; darüber hinaus kommt dem Schuldner eine Bagatellgrenze von $10 \%$ zugute. 1068

Zukünftig wird die „GKV-adäquate“ Auslegung des $§ 17$ InsO betreffend das Vorliegen von Zahlungsunfähigkeit wesentliche Bedeutung erlangen. So steht nach hier vertretener Auffassung ggf. zu erwarten, daß eine wesentliche, durch die Rspr. entwickelte Ausnahme zum kurzen dreiwöchigen Betrachtungszeitraum Bedeutung erlangen könnte, nach der die Liquiditätslücke ohne Eintritt der Zahlungsunfähigkeit auch innerhalb eines längern Zeitraums geschlossen werden kann, wenn die Lücke einschließlich weiter hinzutretender Passiva erstens (nahezu) vollständig geschlossen werden kann und den Gläubigern (darunter indes nicht den Beziehern von Krankengeld) dies nach den Umständen zuzumuten ist. 1069 Denn auf der Aktivseite der Liquiditätsbilanz ist zu bedenken, daß die Einnahmen - staatlich verwaltet - durch Zuweisungen aus dem Gesundheitsfonds grundsätzlich garantiert sind (§ 266 SGB V, § 39 RSAV). Was die Fälligkeit der Zahlungen anbelangt, so gilt für die Liquidität folgendes: Die Auszahlungen erfolgen in Teilbeträgen, die sich insbesondere an den monatlichen Hauptfälligkeitszeitpunkten der beim Gesundheitsfonds eingehenden Beiträge 1070 orientieren, wobei

1067 BT-Drs. 16/9559, S. 17.

1068 S. dazu m.w.N. zur Rspr. Reischl, Insolvenzrecht, 2008 Rn. 93 ff.

1069 A.a.O., Rn. 105.

1070 S. BT-Drs. 16/10609, S. 90: „Hauptfälligkeitszeitpunkte sind insbesondere der erste Bankarbeitstag im Monat für die Beteiligung des Bundes an Aufwendungen (§ 221 Satz 3 SGB V), der Achte eines Monats für die Abschlagszahlung durch die Deutsche Rentenversicherung Bund (§ 255 Abs. 3 Satz 3 SGB V) sowie für Beträge aus Sozialleistungen (§ 23 Abs. 2 Satz 1 SGB IV), der 15. des Monats für sonstige Beiträge einschließlich der Beiträge der Künstlersozialkasse (§ 23 Abs. 1 Satz 4 SGB 
die Zuweisungen für einen Ausgleichsmonat vollständig bis zum 15. des diesem Monats folgenden Monats ausgezahlt werden, § 39 Abs. 4 RSAV. Insbesondere die „sichere Schlusszahlung“ ist hier in den Betrachtungszeitraum ebenso mit einzustellen wie Zahlungsanspruche in Form von Korrekturbeträgen, die sich aus der Strukturanpassung zu den in § 39 Abs. 3 RSAV gem. § 39 Abs. 3a S. 1 RSAV ergeben.

Fraglich ist ferner die Konkurrenz eines Insolvenzeröffnungsgrundes zum Tatbestand der nicht mehr auf Dauer gesicherten Leistungsfähigkeit 1071 , bei dessen gleichzeitigem Vorliegen die Aufsichtsbehörde dem Primat des Schließungsrechts folgend die Kasse schließen soll, $\S 171$ b Abs. 3 S. 2 SGB V. Die bisherigen Anforderungen an die Krankenkasse, wie vergleichbare Mitbewerber entsprechende Regel- und Mehrleistungen zu gewähren und sich dabei annähernd im durchschnittlichen Beitrags- und Leistungsniveau der anderen Krankenkassen halten können, 1072 müssen tw. neu konturiert werden. Denn die genannten Überschreitungsgrenzen, 10\% des landesdurchschnittlichen Beitragsbedarfssatzes der Krankenkassen der Kassenart bzw. 12,5\% des bundesdurchschnittlichen Beitragsbedarfsatzes aller Krankenkassen, können nach der Neuordnung der Finanzierung nicht mehr maßgeblich sein. ${ }^{1073}$ Es muss also erstens auf kassenindividuelle Finanzierungselemente ankommen, ${ }^{1074}$ wobei die Einnahmen selbst unter Einsatz der Rücklage die Ausgaben längerfristig nicht mehr decken können. Zweitens kann bei ggf. erforderlicher Vergleichsbetrachtung im wettbewerblichen Umfeld die Kassenart entsprechend der Lockerung des „Verbundes“ nicht mehr maßgeblich sein. Insgesamt dürfte die prognostiziert dauerhafte Erforderlichkeit eines Zusatzbeitrages am Rande der Sozialgrenze, welche die Liquidität der Kassen mit bestimmt, bestenfalls dann hinnehmbar sein, wenn dem ein besonderes Leistungsangebot gegenüber steht und dieses ausweislich der Mitgliederbewegungen als solches wahrgenommen wird.

Obwohl die danach preis-/leistungsbezogene Wettbewerbs- bzw. Leistungsfähigkeit ${ }^{1075}$ gesichert erscheint, könnte es zwar ausnahmsweise zur Zahlungsunfähigkeit kommen, etwa dann, wenn Zahlungsansprüche im Rahmen der Strukturanpassung nach $\S 39$ Abs. 3 und 3a RSAV - erwartbar oder bereits beschieden - noch nicht realisierbar bzw. fällig sind 1076 oder aber die im Betrachtungszeitraum nicht zu vernachlässigenden Forderungen per Saldo nicht ausreichend bedient werden können. Meist aber wird die

IV), der drittletzte Bankarbeitstag des Monats für Beiträge aus Arbeitsentgelt und Arbeitseinkommen ( 23 Abs. 1 Satz 2 SGB IV) sowie der letzte Tag des Monats für Beiträge aus Renten der Deutschen Rentenversicherung Bund (§ 255 Abs. 3 Satz 1 und 2 SGB V).“

1071 Vgl. $\S 146,153,163,170$ SGB.

1072 Hebeler, Die Vereinigung, Auflösung und Schließung von Sozialversicherungsträgern, NZS 2008, S. 238 (243).

1073 Koch, jurisPK-SGB V, § 146a Rn. 4 m.w.N.

1074 Vgl. oben II.5.1.2.2.

1075 Hebeler, Die Vereinigung, Auflösung und Schließung von Sozialversicherungsträgern, NZS 2008, S. 238 (243).

1076 Was der Durchführung des Zahlungsverkehrs insgesamt und der Liquiditätssituation geschuldet wäre. 
Leistungsfähigkeit als ein Minus zur Zahlungsfähigkeit begriffen bzw. „,in der Regel“ deren Mangel bei gleichzeitiger Zahlungsunfähigkeit attestiert. ${ }^{1077}$ Der Tatbestand der „drohenden Zahlungsunfähigkeit“" (vgl. § 171b Abs. 2 S. 1 SGB V) macht wegen des aufsichtsrechtlichen Instrumentariums im Vorfeld (wenn nicht bereits ein Schließungsgrund vorliegt) und mit Blick auf $\S 172$ Abs. 3 SGB V wenig Sinn: Maßnahmen zur Vermeidung der Zahlungsunfähigkeit sind im Grunde erst dann indiziert, wenn die Zahlungsunfähigkeit droht: Dann aber wäre, da der Tatbestand ja vorläge, sogleich der Insolvenzantrag zu stellen, will man nicht eine negative Vermeidbarkeitsprognose (Erfolglosigkeit der Rettungsfusion und anderer Maßnahmen) vorschalten, die dann wieder bereits an der Leistungsfähigkeit zweifeln lässt.

Eine echte Divergenz zwischen Insolvenztatbestand und Schließung soll nach zuweilen geäußerter Ansicht beim Insolvenztatbestand „Überschuldung“ möglich sein, 1078 dessen zweistufige Prüfung (Überschuldungsbilanz und Fortführungsprognose) eine (zuletzt bis 2013 befristete) Renaissance erfahren hat. ${ }^{1079}$ Zwar ist die Handhabung des Überschuldungsbegriffs wegen der parallelen Wirkungsrichtung des Schließungs- und des Insolvenzgrundes, wegen der zeitlichen Beschränkung des sog. „modifizierten Verschuldungsbegriffs" sowie angesichts des Primats des Schließungsrechts bei gleichzeitiger Fristsetzung zur Stellung des Insolvenzantrages und dem „Anreiz“ der Vermeidung von Amtshaftungsansprüchen 1080 problematisch. ${ }^{1081}$ In der Praxis indes würde eine negative Fortführungsprognose sicherlich auch die Wertung der auf Dauer nicht mehr gesicherten Leistungsfähigkeit enthalten, so daß unter Anwendung der Konkurrenzregel des $\S 171 \mathrm{~b}$ Abs. $3 \mathrm{~S}$. 2 SGB V vorrangig geschlossen würde.

Vor diesem Hintergrund werden aufsichtsrechtlich präventive Maßnahmen besondere Bedeutung erlangen und die (Beaufsichtigung hinsichtlich der) Pflicht zur Auffüllung der Rücklage besondere Bedeutung erhalten.

\subsection{Wettbewerb zwischen privaten Versicherungsunternehmen}

Für das Verhältnis der PKV-Unternehmen zueinander gilt das Wettbewerbsrecht wie v.a. unter I.4.4.3. und 4. beschrieben ohne Einschränkungen. Zwar lässt sich mit der Reform durch das GKV-WSG das Einsickern sozialer und solidarischer Elemente,

1077 Vgl. Bultmann, Die Insolvenzfähigkeit der gesetzlichen Krankenkassen nach dem GKV-OrgWG, MedR 2009, S. 25 (31); Füsser, Das GKV-OrgWG, SGb 2009, S. 126 (128).

1078 Vöcking, Beiträge zur Insolvenzproblematik - aus Sicht der Beteiligten und Betroffenen, GesR 2009, S. 138 (139 f.).

1079 Siehe zuletzt BT-Drs. 16/13980 sowie Annahme in 3. Beratung: BT-P1.Prot. 1/233, S. 26361B 26261C. S. ferner Uwer, Der rechtliche Rahmen der Insolvenz von Krankenkassen, GesR 2009, S. 113 (115 f.).

1080 BT-Drs. 16/9559, S. 20.

1081 Vöcking, Beiträge zur Insolvenzproblematik - aus Sicht der Beteiligten und Betroffenen, GesR 2009, S. 138 (139 ff.). 
bspw. eines Risikoausgleichs, auch in das PKV-System beobachten. ${ }^{1082}$ Dies allerdings hat weder zu besonderen Schutzbestimmungen bzw. Bereichsausnahmen von der Anwendung des Wettbewerbs- und Kartellrechts geführt noch fordert es die tradierte Subsumtion unter den Unternehmensbegriff ernsthaft heraus. ${ }^{1083}$

Dessen ungeachtet entsteht zwischen den PKV-Unternehmen im Zeichen des GKVWSG neuer Konkurrenzdruck, denn der Wechsel innerhalb der PKV wird erleichtert, jedoch zuvorderst für die ab 2009 geschlossenen Verträge. Das den Wettbewerb antreibende Kernelement ist die gesetzlich verbriefte Mitnahme der Altersrückstellungen, wenngleich dies nur im Umfang der Bemessung am Basistarif möglich ist. Bis dato war die mangelnde Portabilität der Altersrückstellungen jedenfalls aus Sicht der PKVVersicherten ein Hindernis, das Versicherungsunternehmen zu wechseln, ggf. kumuliert mit der Abschlußfreiheit (auch) dieser Unternehmen und der Möglichkeit, Leistungsbereiche auszuschließen oder Risikozuschläge zu erheben. Seitens der PKV-Unternehmen wurde überdies das „Wechsel-Zeitfenster“ in Form einer Frist vom 1. Januar bis zum 30. Juni 2009 als ebenso ,riskant“ wie interessant eingestuft, innerhalb derer (junge) Versicherte aus dem Angebotstarif eines anderen PKV-Unternehmens in das eigene (mit Kontrahierungszwang) wechseln durften, zwar (zunächst) im Basistarif, 1084 doch ggf. mit der von PKV-Unternehmen später forcierten Option, dort in den Angebotstarif „aufzusteigen“.

Auch die Konkurrenz um den Vertreib von Krankenhaus-Zusatzversicherungen kennzeichnet einen lukrativen Markt. Hier geht es mit Blick auf $\S 194$ Abs. 1a SGB V zugleich um einen Vertrags- und Kooperationswettbewerb mit gesetzlichen Krankenkassen. Denkbar ist dabei auch ein strategisches Marktverhalten von PKVUnternehmen, die eine BKK errichtet haben, durch deren Fusion oder Kooperation mit anderen BKKen Krankenhaus-Zusatzversicherungsmarkt über § 194 Abs. 1a SGB V zu eröffnen.

\subsection{Wettbewerb zwischen gesetzlichen Krankenkassen und PKV-Unternehmen}

Das (Konkurrenz-) Verhältnis der gesetzlichen Krankenkassen zu den PKVUnternehmen entfaltet seine Bedeutung vor allem dort, wo im Gefolge hinreichender Überschreitung der Jahresarbeitsentgeltgrenze die Möglichkeit zur freiwilligen Versicherung besteht. Wie bereits in der Entscheidung AOK-Bundesverband ${ }^{1085}$ und darüber hinaus im Schrifttum ${ }^{1086}$ zum Ausdruck kommt, ist nach verbreiteter, im Rahmen der

1082 Vgl. nochmals oben I.1.1.

1083 Siehe auch Roth, Kartellrechtliche Aspekte der Gesundheitsreform nach deutschem und europäischem Recht, GRUR 2007, S. 645 (659).

1084 Siehe Art. 44 Ziff. 5 b) GKV-WSG (BGB1. I 2007, S. 469) betreffend $\S 12$ b S. 2 VAG.

1085 Siehe oben II.3.1.5.

1086 Roth, Kartellrechtliche Aspekte der Gesundheitsreform nach deutschem und europäischem Recht, GRUR 2007, S. 645 (658 f.). 
Untersuchung tw. kritisierter Ansicht allein das ausschnittweise direkte (Versicherungs-) Konkurrenzverhältnis zwischen Krankenkassen und PKV-Unternehmen geeignet, eine Unternehmenseigenschaft der gesetzlichen Krankenkassen zu begründen. Damit berühren die gesetzlichen Krankenkassen die im PKV-Bereich vorfindbaren Marktstrukturen; insoweit besteht ein nur partielles und unvollkommenes Konkurrenzverhältnis. 1087

Gleichwohl erfährt auch im Verhältnis der Kostenträger beider Systeme zueinander wettbewerbsschädigendes Verhalten beider Seiten eine dezidierte Kontrolle, bei welcher der sozialrechtliche Kontrollmaßstab dominiert und der Rechtsstreit im Sozialgerichtsweg zu verfolgen ist. ${ }^{1088}$ Eine Anwendung des UWG ist jedenfalls durch $§ 69$ SGB V zwar nicht völlig ausgeschlossen, jedoch nur in seltensten Fällen nicht mir der Frage der Konturierung einer Angelegenheit der gesetzlichen Krankenkassen verbunden, soweit es sich nicht um ,schlicht“ unlautere Handlungen geht.

Darüber hinaus besteht ein kooperatives Verhältnis zwischen Krankenkassen und PKV-Unternehmen im Hinblick auf die Vermittlung privater Zusatzversicherungen für die Krankenhausbehandlung durch die Krankenkassen. ${ }^{1089}$ Je nach Perspektive führt dies wiederum zur Konkurrenz unter den PKV-Unternehmen, aber auch die Vermittlungstätigkeit gesetzlicher Krankenkassen unterminiert die Akquisition seitens der für die PKV tätigen Versicherungsmakler, so daß es - untechnisch gesprochen - zur Konkurrenz zwischen „Fremd- und Eigenvermittlung“ betreffend dasselbe Versicherungsprodukt kommt.

Als ein der PKV tendenziell ähnliches Versicherungselement schließlich ist nochmals der GKV-Wahltarif für Kostenerstattung bei „Chefarztbehandlung“ und „Zweibettzimmer" zu betrachten. Wichtig ist dabei, daß Kostenerstattung insoweit - mindestens - den Bereich der Krankenhausleistungen insgesamt erfassen muss. 1090 Darüber hinaus ist ebenso eindeutig, daß der gesetzliche Leistungskatalog die Chefarztbehandlung nicht ausschließt, sondern diese im Gegenteil im Einzelfall sogar erfordert. ${ }^{1091} \mathrm{Zu}$ diskutieren indes gilt, ob es sich vor diesem Hintergrund um eine unzulässige Ausweitung des Leistungskatalogs der GKV handelt. Daß hier oftmals der Vergleich mit klassischen Zusatzversicherungen angestellt wird, die sonst nur die PKV anbietet, ${ }^{1092}$ ist jedenfalls kein zwingendes Argument gegen die rechtliche Zulässigkeit, soweit eine gewisse Konvergenz bzw. Konkurrenz gesetzlich intendiert ist. Letzteres lässt sich jedenfalls ausweislich der Gesetzesbegründung schwer von der Hand weisen:

1087 Dazu unter II.3.1.6.

1088 Vgl. dazu oben II.5.1.3.1 sowie die zahlr. N bei BGH, Beschl. v. 9.11.2006, GRUR 2007, S. 535 (536); siehe ferner Dieckman/Wildberger/von Quast, Rechtswegzuweisung nach § 51 Abs. 2 SGG bei unlauterem Wettbewerb gesetzlicher Krankenkassen, NZS 2005, S. 187 ff.

1089 Ausführlich oben II.2.1.4, sowie vorstehend II.5.2.

1090 S. II.2.1.6.

1091 Schlegel (2007), jurisPK SGB V, § 53 Rn. 113.

1092 Vgl. Lang, in: Becker/Kingreen, SGB V (2008), § 53 Rn. 15. 
„Diese Tarifmöglichkeit stärkt die Wettbewerbsposition der gesetzlichen Krankenkassen gegenüber der privaten Krankenversicherung.“"1093

$\mathrm{Da}$ die PKV nach anderen Regeln operiert und etwa wegen der Bindungen an die Kalkulationsverordnung „Nachteile“ erlangt, ist eine höchstrichterlicher Klärung harrende Frage verfassungsrechtlicher Rechtfertigung, ggf. verfassungskonformer Interpretation. Deutlich wird jedenfalls, daß sich die Frage der Zulässigkeit nach Art und Umfang auf der Ebene der Wirtschaftlichkeit und dem dahingehenden Prinzip des $\S 12$ Abs. 1 SGB V zuspitzt. ${ }^{1094}$ Dabei aber ist zu bedenken, daß $§ 53$ Abs. 9 SGB V eine Quersubventionierung ausschließt, und zwar auch zwischen den Wahltarifen. Die Anforderungen sind daher hoch, und jedenfalls unter Gesichtspunkten der Kostenbelastung anderer, nicht im Wahltarif Versicherter, erfolgt eine Begrenzung, die mit Blick auf eine behauptete Erosion des Solidarprinzips für bedeutsame Abmilderung sorgt. Insofern kann, will man $§ 53$ Abs. 4 SGB V jenseits verfassungsrechtlicher Fragen einen maßgeblichen Anwendungsbereich belassen, nicht gefordert werden, daß es gegenüber der Erbringung von Sachleistungen nicht zu Mehrkosten kommen wird. Das bringt der Gesetzgeber selbst deutlich zum Ausdruck:

„Beispielsweise wäre es möglich, dem Versicherten den 2,3-fachen Satz nach GOÄ/GOZ zu erstatten. Für die Mehrkosten, die dies gegenüber Sachleistungen bedeutet, muss die Kasse eine entsprechend kalkulierte Prämienzahlung des Versicherten einfordern.“1095

Die „Probebohrung“1096 Wahltarif für stationäre Leistungen einschließlich Chefarztbehandlung und Zweibettzimmer ist daher - wie die Auseinandersetzungen zeigen 1097 - durchaus wettbewerbsrelevant, wenngleich angesichts der Kalkulationserfordernisse und Rechtsunsicherheiten in begrenztem Rahmen.

1093 BT-Drs. 16/3100, S. 109.

1094 S. Isensee, Wahltarif "Krankenhauskomfort" - Chefarztbehandlung und Ein-/Zweibettzimmer als Wahlleistungen de Kassen - Sicht des Sozial- und des Verfassungsrechts, NZS 2007, S. 449 (451).

1095 BT-Drs. 16/3100, S. $108 \mathrm{f}$.

1096 S. Isensee, Wahltarif "Krankenhauskomfort" - Chefarztbehandlung und Ein-/Zweibettzimmer als Wahlleistungen de Kassen - Sicht des Sozial- und des Verfassungsrechts, NZS 2007, S. 449 (450).

1097 Vgl. oben II.2.1.6. 


\section{Wesentliche Ergebnisse - Wahl und Wettbewerb im regulierten System}

\section{Zur Einführung und Kontrolle marktförmiger Strukturen durch Recht}

\section{1.}

- Auch nach den jüngsten Reformen präsentiert sich Wettbewerb im Bereich der Krankenhausversorgung als ein in besonderem Maße regulierter Wettbewerb.

- Er ist weithin unvollkommen und als solcher Teil eines gemischt regulativwettbewerblich orientierten Systems im Gesamtzusammenhang bundes- und landesrechtlicher sowie kollektiv- und selektivvertraglicher Normen.

- Wettbewerb in der Krankenhausversorgung bedeutet mangels echten Preiswettbewerbs (derzeitiges DRG-System) v.a. „Wettbewerb um Strukturvorteile im regulierten System“, beginnend etwa mit der Konkurrenz um die abrechnungsprivilegierte Planposition (Wettbewerb um den „Systemzutritt“).

- Wettbewerb um den Erhalt der privilegierten Position und Systemverbleib etwa wird durch Mengenanforderungen (Mindestmengen) gesteuert und beflügelt die Konkurrenz um Absatzanteile im begrenzten Nachfragesegment.

- Wahlrechte wie das Recht nach § 39 Abs. 2 SGB V sind nur unvollkommen verankert und regulatorisch bzw. planbedingt begrenzt.

- Das Selektivvertragsprinzip in der GKV wird letztlich nicht um der Freiheit der Leistungserbringer willen, sondern aus Gründen der Allokation der Ressourcen zugunsten der gesamten Solidargemeinschaft verfolgt.

- Wo dessen Ergebnisse nicht zufrieden stellen, dominiert wieder die Regulierung (vgl. § 116b Abs. 2 SGB V) oder es wird „kollektivierend“ durch Inhaltsvorgaben (vgl. § 140a Abs. 1 SGB V) oder mittels Kontrahierungszwang bzw. -macht (vgl. § 73b Abs. 1, § 73c SGB V) ebenso regulatorisch befördert wie durch andere systemische Privilegierungen und -verschränkungen. 1098

- Der Erhalt der staatlichen Investitionsfinanzierung sowie die Begrenzung bzw. der Ausschluss eines generellen selektiven Preiswettbewerbs unter im übrigen gleichen Bedingungen begrenzen im wesentlichen wettbewerbliche Beziehungen zwischen Krankenkassen- und Krankenhäusern.

- Vor allem im Gefolge des GKV-WSG ist eine „Konvergenz“ der gesetzlich solidarischen und der Privatversicherung erkennbar. 
1.2.

- Auch wenn im ordnungspolitischen bzw. gesamtsystemischen Rahmen regulatorische Elemente dominieren, bestehen en detail doch mannigfache Wettbewerbsbeziehungen, selbst wenn die dahinter stehenden Vergütungsvolumina einen deutlich geringeren Teil ausmachen bzw. die Krankenhausversorgung nur mittelbar andere Wettbewerbsverhältnisse bestimmt.

- Insbesondere im (sozialversicherungs-)rechtlichen Dreiecksverhältnis müssen die wettbewerbsrelevanten Beziehungen multipolar gedacht werden: Der Wettbewerb der Krankenkassen etwa ist Mitgliederwettbewerb, z.B. mittels beworbener Versorgungsangebote (IV, DMP), die im Kooperationswettbewerb mit den Krankenhäusern ausgehandelt wurden.

- Der mehrfach revidierte $\S 69$ SGB V ist ebenso Ausdruck einer beteiligtenspefizisch erforderlichen Analyse der Wettbewerbsbeziehungen wie gemischt regulatorisch-wettbewerblicher Steuerung durch partiellen Einlass ausgewählter Normen des GWB.

- Soweit nach maßgeblicher sozialrechtlicher Betrachtung anwendbar, tragen das bundesrechtlich verankerte Wettbewerbsrecht im engeren Sinne und das landesrechtlich verbindliche Berufsrecht in den Beziehungen der Krankenhäuser untereinander zum Schutz der wachsenden Wettbewerbsbeziehungen bei. Der sozialund krankenhausrechtlich vermittelte Wettbewerbsdruck führt dazu, daß das Wettbewerbsrecht zunehmend einen neuen Wirkbereich erhält.

- Primäres und sekundäres Gemeinschaftsrecht gewinnen im Zeichen zunehmend marktförmiger Strukturen selbst innerhalb der nationalen ,solidarischen Wettbewerbsordnung" stärker an Einfluß.

- Das Zusammenspiel zwischen nationaler Wettbewerbsorientierung und dem hinsichtlich seiner Anwendung vorrangigen europäischen Wettbewerbsrecht wird die marktförmige Entwicklung weiter beflügeln.

- Schutz gegen kartellwidriges Handeln der Krankenkassen wird trotz Ausschlusses des $\S 1$ GWB nach $\S 69$ SGB V ggf. durch Art. 81 EGV vermittelt; in der „solidarischen Wettbewerbsordnung“ agieren die Krankenkassen im Hinblick auf besondere Selektivverträge ausnahmsweise als Unternehmen.

- Die Ambiguität eines gemischt wettbewerblich-regulierten Systems kommt ferner dadurch zum Ausdruck, daß Krankenkassen teilweise nicht nur als Unternehmen, sondern u.U. zugleich als öffentlicher Auftraggeber tätig werden.

- Im Zeichen multipolarer Wettbewerbsbeziehungen lassen sich normativ verankerte „Steuerungswirkungen“ zumeist nur relativ bemessen. Steuerungsinstrumente in bestimmten Wettbewerbsverhältnissen sind Teil eines Kausalitätsge- 
flechts mit mittelbarer Einflussnahme auf Beziehungen zu weiteren Akteuren und treten in Wechselwirkung mit anderen steuerungspolitischen Maßnahmen.

- Normative Steuerung im Krankenhaussektor durch Wettbewerb ist daher nur in ihrer Mehrdimensionalität zu begreifen. Dabei kommt die Komplexität der Wettbewerbsbeziehungen im Spannungsfeld zwischen Markt und Regulierung bereits durch divergierende Interpretationsansätze des $§ 69$ SGB V zum Ausdruck.

- Daß ein „Zielkonflikt zwischen Wettbewerbsrecht und Gesundheitspolitik“ im Bereich der Zusammenschlüsse von Krankenhäusern nicht besteht (OLG Düsseldorf), entspricht dem Befund, daß Fusionen nicht in hinreichend engem Zusammenhang mit Handlungen in Erfüllung des öffentlich-rechtlichen Versorgungsauftrags der Krankenkassen stehen.

\section{4 .}

- Der Markt für Krankenhausleistungen ist als Absatzmarkt an der Nachfrage durch den Patienten, auch den gesetzlich Versicherten, ausgerichtet. Die rechtliche Ausgestaltung und praktische Bedingtheit des Krankenhauswahlrechts der Patienten dient dem allerdings nur unvollkommen.

- Bedeutender Wettbewerbsparameter ist die Behandlungsqualität. Hier setzten neue Mechanismen wie die Veröffentlichung von Qualitätsberichten im Internet und vergleichende Empfehlungen seitens der Kasse als normativ fixierte Steuerungsmechanismen fördernd an.

- Mittelbar wird der Absatzmarkt über die Angebote der Krankenkassen gesteuert, sei es durch entsprechende Tarife, besondere Versorgungsformen, die Vermittlung privater Zusatzleistungen für die Krankenhausbehandlung oder krankenhausspezifische Sonderleistungen.

- Das Preisrecht spielt für die Konkurrenz der Krankenhäuser untereinander nur bedingt eine Rolle. Es eröffnet in Abhängigkeit von der Art der Leistung nur geringe Gestaltungsspielräume.

- DRG in ihrer nationalen Ausprägung sind mangels autonom verhandelbarer Bewertungskomponente kein an der wettbewerblichen Preisgestaltung orientiertes Instrument.

- Zunehmend Bedeutung erlangen Strategien der Einweisungssteuerung durch Krankenhäuser. Während allerdings die Einflußnahme über die Trägerschaft von MVZ weithin zulässig ist, sind ,innovativen“ Strategien in Form von Einweisungspauschalen (im Rahmen des Managed Care) stärker Grenzen gesetzt.

- Letztere sind im Beziehungsgeflecht zwischen Sozial- und - tw. konträrem - Berufsrecht allerdings schwer zu konturieren. Verstärkt kooperatives und die Versorgungssektoren verzahnendes Handeln erhöht den „Druck“ auf das Berufsrecht und läßt immer deutlicher Konfliktfelder zutage treten, die nach einer grundlegenden Betrachtung des Verhältnisses beider Regime zueinander verlangen. 
- Angesichts reduzierter berufsrechtlicher Restriktionen spielt die Krankenhauswerbung eine zunehmend bedeutsame Rolle.

- Im Zeichen zunehmenden Wettbewerbs durch neue Versorgungsformen eröffnet der Selektivvertragswettbewerb auch einen Wettbewerb der „Binnenkooperation“ der Leistungserbringer um effiziente Vernetzung.

- Konzentrationsprozesse infolge struktureller Reorganisation und dem Bemühen um Effizienz und Spezialisierung führen zu Versorgungsproblemen in der Fläche und im Notfallbereich. Das rechtliche Instrumentarium, dem entgegenzusteuern, ist ausbaubedürftig.

- Kommunale Verlustausgleiche für besonders an der Daseinsvorsorge orientierte öffentliche Häuser sind bei Erfüllung der eigens dafür aufgestellten europarechtlichen Anforderungen zulässig.

- Eine Symbiose von plankrankenhäuslichen und privatklinischen Organisationseinheiten zur Lösung der Bindung an öffentlich-rechtliche Preisvorschriften widerspricht Planungsrecht.

1.6 .

- Im Selektivvertragswettbewerb mit den Krankenhäusern entspricht das Handeln der Krankenkassen zunehmend marktförmigen Strukturen. Sie agieren in diesem Bereich mitunter als Unternehmen i.S.d. Art. 81 f. EGV.

- Es erscheint mit Blick auf besondere wirtschaftliche und systemische Privilegierungen sowie die zunehmende, oft auch verzahnte Diversifikation von Prämienund Angebotsstrukturen ebenso zulässig wie geboten, auch innerhalb der GKV Marktstrukturen zu identifizieren, die der Kontrolle marktförmigen Handelns bedürfen, wie sie für (private) Unternehmen in herkömmlicher Weise bekannt ist.

- Dies hat eine Abkehr von einer einheitlichen Betrachtungsweise von Gesamtversicherungs-Märkten zur Folge, die allein im Hinblick auf den Kreis der freiwillig Versicherten Marktstrukturen identifiziert.

- Integrationsverträge sind im Zeichen der europäischen Vorgaben nach Maßgabe des $\S 69$ Abs. 2 S. 1 Hs. 2 SGB V in Abhängigkeit von der konkreten Ausgestaltung öffentlich auszuschreiben. Bei qualifizierter flächendeckender Versorgung mit Budgetverantwortung und unter Einschluss der stationären Versorgung ist dies der Fall. Dadurch allerdings entstehen zusätzliche, nicht unerhebliche Transaktionskosten.

- Ausschreibung einerseits und die Kontrolle unternehmerischen Handelns andererseits sind besonderer Ausdruck der Ambiguität marktorientierter Strukturen im GKV-System. Gerade umfassende Ausschreibungspflichten stehen einem von 
der Kreativität der Leistungserbringerseite her induzierten eigenen Angebotswettbewerb in größerem Umfang entgegen.

- Selbst im regulierten GKV-System herrscht mit Blick auf das Selektivvertragsrecht kein Vertragstypenzwang, auch wenn die Gestaltungsspielräume in besonderem Maße systemimmanenten Beschränkungen unterliegen.

\section{7.}

- Im Vergleich mit den gesetzlichen Krankenkassen ist der (originäre) Versicherungsmarkt der PKV-Unternehmen sehr viel stärker ausgeprägt und unterliegt echten Marktbedingungen.

- Andererseits besteht dieser losgelöst von - selten vorfindbaren - Vertragsbeziehungen der PKV-Unternehmen zu Krankenhäusern als Leistungserbringern. Dies ist den Strukturen des deutschen PKV-Systems geschuldet, für welches im übrigen keine der GKV vergleichbaren Regularien für integrierte Versorgung in puncto (Dispens vom) Gebührenrecht, Qualitätssicherung etc. bestehen.

- Angesichts genereller Erstattung der Kosten für allgemeine Krankenhausleistungen und im Einklang mit privatversicherungsrechtlichen Strukturen bilden Versorgungskonzepte zwischen PKV-Unternehmen und Krankenhäusern die Ausnahme und eignen sich nicht als Wettbewerbsparameter.

- Ungeachtet dessen ist ein wettbewerbsorientierter Fusions- und Konzentrationsprozeß sowohl bei den gesetzlichen als auch den privaten Kostenträger zu konstatieren, der im PKV-Bereich materiell-rechtlich der Kontrolle am Maßstab des GWB, für die gesetzlichen Krankenkassen hingegen allein den Vorgaben des SGB V, unterliegt.

- Im Zeichen strittiger Wahltarife zur Kostenerstattung bei stationärer Versorgung inklusive Zweitbettzimmer und Chefarztbehandlung kommt es in gewissem Umfang ebenso zur Konvergenz der Systeme wie durch einen Kontrahierungszwang im Bereich der privaten Basisversicherung und begrenzte Risikoausgleichsmechanismen in der PKV.

- Im Hinblick auf die Vermittlung stationärer Zusatzversicherungen kommt es zu einem Wettbewerb um Kooperationspartner, der sich sowohl innerhalb von GKV und PKV als auch versicherungsübergreifend abspielt. 


\section{Steuerungseffekte und Interdependenzen}

\subsection{Finanzierung, Ausgaben und Effizienz}

- Der Schwerpunkt normativer Steuerung der Krankenhausversorgung durch Wettbewerb liegt im Bereich der (im case-mix günstigen) Absatzsteigerung, vermittelt v.a. durch Qualität.

- Als Katalysator sowohl der Absatzoptimierung als auch des Erhaltes des Abrechnungsprivileges fungieren Mengenssteuerung bzw. die Leistungskonzentration bei entsprechend leistungsstarken Häusern.

- Anstelle selektiven Preiswettbewerbs dienen (tendenziell) einheitliche Finanzierungsgrundlagen dazu, eine kostenregulierte (landes-)einheitliche Ausgangsposition für Wettbewerb mittels anderer Parameter zu schaffen.

- Fehlender Preiswettbewerb im DRG-System macht komplexe, regulativ korrigierende Eingriffe zur Austarierung des Vergütungssystems zugunsten der Versorgungssicherheit und Qualitätssicherung erforderlich.

- Da und soweit Investitionskosten im DRG-System nicht „eingepreist“ sind, sondern der dualen Finanzierung folgend planbezogener Förderung durch unterschiedliche Hoheitsträger unterliegen, fehlt es insgesamt nicht nur an Preiswettbewerb, sondern überdies an „echten“ (und bereits bundes-) einheitlichen Wettbewerbsgrundlagen für einen Wettbewerb jenseits des Parameters „Preis“.

- Ein „Investitionsbedarfsausgleich“ erfolgt nicht.

- Gewisse Möglichkeiten zur Preisgestaltung hingegen bestehen im Rahmen eines begrenzten Selektivvertragswettbewerbs.

- Die Opportunitätskosten neuer Versorgungsmodelle und der Aufwand der Gestaltung des Selektivvertragswettbewerbs sind nicht zu unterschätzen.

- Im Rahmen der IV sind Integrations-, Koordinations- und Dokumentationsaufwand vergütungsfähig. Erst seit Wegfall der Anschubfinanzierung eröffnen sich weitere Preisgestaltungsmöglichkeiten durch Ausgliederung der Leistungen aus dem Krankenhausbudget.

- Unter enormem Kosten- und Rationalisierungsdruck entseht ein Verdrängungswettbewerb, dem sich die Häuser oftmals in struktureller Reorganisation stellen und so einen Wettbewerb der Organisations- und Gesellschafts- und Geschäftsmodelle befördern, der auch Formen des „,in-“ oder „,outsourcing“ oder einer PPP erfasst. 


\subsection{Zusammenwirken und Wechselwirkungen: „Regulierungsverflechtung“}

- Normative Steuerungsansätze lassen sich kaum in isolierter Wirkungsrichtung, sondern nur in ihrer Einbindung in multipolare Wettbewerbsverhältnisse und im Zusammenwirken mehrer Maßnahmen und des Verhaltens aller betroffenen Akteure bewerten.

- Im föderalen System besteht ein hohes Maß an „Regulierungsverflechtung“ (vgl. $\S 116 b$ Abs. 2 SGB V; § 39 Abs. 2 SGB V).

- Dies kann, muß aber nicht Ausdruck einer Zielverschränkung bzw. -kollision sein, wie sie etwa im Bereich des mit Planungs- und Bedarfserwägungen „belasteten" Rechts des Patienten, das Krankenhaus zu wählen, hervorsticht.

- Vor diesem Hintergrund ist, wie das KHRG verdeutlicht, einem echten Monistischen System in Deutschland auf absehbare Zeit voraussichtlich kein Erfolg beschieden.

- Sowohl im Zuge der unter 1.1. beschriebenen Tendenzen als auch im Hinblick auf die gesteigerte Marktmacht „kooperierender“ Vertragsgemeinschaften ist eine „Kollektivierung“ selektivvertraglicher Strukturen bzw. eine Kollektivierung der im Selektivvertragswettbewerb erzielten Ergebnisse zugunsten wohlfahrtstaatlicher Allokation zu erkennen.

- Planung und Wettbewerb können einander ebenso hemmen, wie Wettbewerb hinsichtlich der Planposition und um deren Erhalt bestehen kann.

- Im Bereich der Wahl des Krankenhauses werden sowohl ein freiheitlich wettbewerbsorientierter als auch ein regulatorisch und planungsorientiert zuweisender Ansatz gleichzeitig verfolgt. Das Wahlrecht jedoch erweist sich damit als unvollkomen.

- Nach Abschluss der Konvergenzphase bei den DRG wird die auch nach Erlaß des KHG in partiell modifizierter Form fortexistierende dualistische Finanzierung grundsätzlich permanent zu überdenken sein.

- Entlastungswirkungen im Zeichen des Endes der Konvergenzphase werden durch einen nicht unerheblichen Entwicklungs- und Implementierungsaufwand der Investitionspauschalen ab 2012 teilweise aufgezehrt.

- Das System ist hochkomplex (vgl. nur $\S \S 17$ a bis 17d SGB V). Die in $\S 10 \mathrm{KHG}$ verankerten Schritte zur Entwicklung der optionalen Investitionsfinanzierung durch leistungsorientierte Investitionspauschalen nach Maßgabe von Investitionsbewertungsrelationen sind kein Beitrag zur Deregulierung.

- In dem vorstehend beschriebenen Regulierungsgeflecht bieten nur optimierte, langfristig kooperativ angelegte PPP-Modelle eine Chance, das Investitionsdefizit zu begrenzen. 


\subsection{Wettbewerbsverzerrungen und exogene Stützung}

- Das dualistische Finanzierungssystem wirkt ungeachtet seiner Bedeutung für die Sicherstellung regionaler Versorgung tendenziell wettbewerbsverzerrend, da der in den Ländern unterschiedlich zugestandene Investitionsaufwand naturgemäß weder Erlösbestandteil noch unmittelbar erfolgsabhängig ist.

- Die Aufrechterhaltung der dualistischen Finanzierung nach Ende der DRGKonvergenzphase kann zu merklichen standortspezifischen Wettbewerbsverzerrungen führen, wenn der landeseinheitliche Preis und die Investitionsförderung in einzelnen Ländern entsprechend gering ausfallen.

- Unter den gegebenen Bindungen wird daher ein „Konvergenzdruck“ in Richtung bundeseinheitlicher Preise erfolgen.

- Ein zentrales Problem regulierten Wettbewerbs besteht naturgemäß darin, wie mittelfristig wohlfahrtsfördernde Investitionskosten im solidarischen Wettbewerbssystem internalisiert werden können.

- Daß der Monismus die Investitionskosten in diesem Sinne richtig abbilden kann, ist keinesfalls garantiert, hängt jedenfalls von der Ausgestaltung und Vergütungsgarantie, d.h. davon ab, inwiefern erhöhte Ausgabenvolumina tatsächlich bei den Krankenhäusern ankommen. Gleichzeitig würde es andere Formen der Regulierung bedürfen, ggf. auch in Form monetärer Anreize mit tendenziell wettbewerbsverzerrender Wirkung, um eine flächendeckende und Notfallversorgung jenseits rein ökonomischer Marktmechanismen sicherzustellen.

- Wettbewerb bedeutet vielfach Verteilungswettbewerb innerhalb des Budgets oder gar Konkurrenz um Wiedererlangung „abgezogener“ Budgetanteile. Die vormalige Anschubfinanzierung der Integrierten Versorgung ist Beispiel einer solchen Verzerrung.

- Ein grundsätzliches Problem „morbiditätsorientierter“, an Diagnosen ausgerichteter Systeme besteht in Tendenzen des „Up-Codings“ oder anderweitiger Manipulation, oft fälschlich als „Right-Coding“ verklärt. Hier ist eine Vermeidung von „Fehlanreizen“ und -verhalten ebenso wie eine Sanktionierung unrechtmäßiger „Input-Manipulation“ gefragt.

- In komplexen Systemen stellt das Erfordernis der Kohärenz den Gesetzgeber oft vor besondere Herausforderungen. Ziel- und Politikverflechtung bedeuten auch Steuerungsverflechtung mit hohem Regulierungsaufwand zur Wahrung der Kompatibilität der Regelungskomponenten, der Kohärenz und „Systemimmanenz" sowie zur Abwehr von Fehlanreizen. Solche Systeme sind daher in hohem Maße „selbstreferentiell“.

- Eine besondere Form der ohnehin wettbewerbsbegrenzenden Preisregulierung ist die „Beteiligung“ einzelner Akteure an der GKV-weiten Ausgabenfinanzierung in Form vielfältiger Finanzierungskürzungen. 


\section{Fazit}

- Ein gemischt wettbewerblich-regulatorischer Ansatz normativer Steuerung der Krankenhausversorgung ist im Kontext sozialstaatlich und grundrechtlich determinierter Gewährleistungsverantwortung in Diensten der allgemeinen Gesundheitsversorgung alternativlos.

- Dieser Ansatz ist der Sicherstellung der Versorgung ebenso verpflichtet wie dem Einsatz und der Kontrolle effizienz-, wirtschaftlichkeits- und angebotsoptimierender marktförmiger Anreizstrukturen; muß grundrechtlich fundierten wirtschaftlichen Freiheiten der Akteure hinreichend Rechnung tragen.

- Die „Steuerungsmix“ verliert an Ausgewogenheit, Verlässlichkeit, und Operationalität und damit insgesamt an Zielbezug und ggf. erforderlicher Rechtfertigungsfähigkeit, wenn er sich weithin auf einen Wettbewerb um Strukturvorteile im regulierten System beschränkt und Wahlrechte als unvollkommene oder unechte Wahlrechte erscheinen lässt.

- Regulierungsverflechtung und Mehrdimensionalität im Kausalitätsgeflecht der Anreizstrukturen lassen normative Steuerung immer komplexer erscheinen. Abgesehen von unmittelbaren Mittelkürzungen bzw. „Sanierungsbeiträgen“ wird gerade positive Verhaltenssteuerung zunehmend indirekter.

- Im Bereich der Krankenhausfinanzierung lautete die Frage - aus rechtlicher Sicht - nicht zwingend Monismus oder Dualismus. In einem monistischen System etwa sind regional abgestufte besondere Investitionszuschläge zum Leistungsentgelt zur Sicherung flächendeckender und notwendiger Versorgung ebenso denkbar, wie ein mit hinreichenden Finanzmitteln ausgestattetes, nach stärker einheitlichen Grundsätzen ausgerichtetes System der länderverantworteten Investitionsfinanzierung zum Abbau von Wettbewerbsverzerrungen und Komplexität beitragen könnte.

- Effektive normative Steuerung ist mehr als die v.a. formal koordinierte Einbindung von Partikularinteressen in das System und Wettbewerb mehr als diese Summe instrumenteller Anreize. Vielmehr muss sie eine enge Abstimmung der verschiedenen Hoheitsträger abbilden, die politische, akteursbezogene und versorgungsspezifische „Sektoren“ integriert. 


\section{Literatur}

Altendorfer, Reinhold/ Merk, Wolfgang/ Jensch, Ingolf: Das Medizinische Versorgungszentrum, Frankfurt am Main 2004.

Amelung, Volker Eric u.a. (Hrsg.): Integrierte Versorgung und Medizinische Versorgungszentren. Von der Idee zur Umsetzung, Berlin 2006.

Andreas, Manfred: Medizinische Versorgungszentren, ArztRecht 2005, S. 144-151.

Anonymus: Unlautere Methoden im Gesundheitswesen - Wettbewerbszentrale berichtet über Tätigkeit gegen Ärzte und Krankenhäuser, KHuR 2006, S. 3739.

Augurzky, Boris/ Tauchmann, Harald/ Werblow, Andreas/ Felder, Stefan: Effizienzreserven im Gesundheitswesen. Hrsg.: Rheinisch-Westfälisches Institut für Wirtschaftsforschung (RWI): Materialien, Heft 49, Private Krankenversicherung, München 2002.

Axer, Peter: Einbeziehung der PKV in die GKV. Standard- und Basistarif als Gegenstand der Sicherstellung in der vertragsärztlichen Versorgung, MedR 2008, S. 482-492.

Bach, Peter/ Moser, Hans: Private Krankenversicherung, München 2002.

Ballast, Thomas: Integrierte Versorgung zwischen Anspruch und Wirklichkeit, ErsK 2004, S. 221-224.

Balzer, Miriam: Aktuelle Rechtsprobleme aus dem Arzt- und Klinikwerberecht, KHuR 2003, S. 87-99.

dies.: Arzt- und Klinikwerberecht, Berlin u.a. 2004.

Bangard, Annette: Fusionskontrolle bei Krankenhäusern, Präsentation zum Symposium „Krankenhaus und Recht“, 9. November 2005, S. 9, abrufbar unter www.stamas.bayern.de (Krankenhaus).

Bauer, Stefan: Rechtssicherheit bei der Finanzierung gemeinwirtschaftlicher Leistungen?, EuZW 2006, S. 7-11.

Becker, Stefan: Das Recht der Hochschulmedizin, Berlin/Heidelberg 2005.

Becker, Ulrich: Gesetzliche Krankenversicherung zwischen Markt und Regulierung, JZ 1997, S. 534-544.

ders.: Rechtliche Rahmenbedingungen der integrierten Versorgung, NZS 2001, S. 505-514.

ders.: Bayerisches Kommunalrecht, in: Becker, Ulrich/ Heckmann, Dirk/ Kempen, Bernhard/ Massen, Gerrit, Öffentliches Recht in Bayern, 3. Aufl., München 2005, 2. Teil.

ders.: EU-Beihilfenrecht und soziale Dienstleistungen, NZS 2007, S. 169-176.

Becker, Ulrich/ Kingreen, Thorsten: SGB V, Kommentar, München 2008.

Beckschäfer, Hartmut: Die Wahltarife nach $\S 53$ SGB V - Bilanz und Ausblick aus der Sicht des Bundesversicherungsamtes, ErsK 2008, S. 438-441.

Benner, Volkmar: Disease-Management-Programme. Unterschiedliche Ansätze von privater und Gesetzlicher Krankenversicherung, Deutsches Ärzteblatt, Vol. 100, 2003, Heft 3, S. A82-A84.

Beule, Claudia: Rechtsfragen der integrierten Versorgung, Berlin/Heidelberg 2003.

dies: Integrierte Versorgung nach neuem Recht, GesR 2004, S. 209-214.

Binder, Stefan, u.a. (Hrsg.): Sozialgerichtsgesetz, Handkommentar, Baden-Baden 2003 (zitiert: Hk-SGG).

Boetius, Jan: Gegen den Wind - der Basistarif der Gesundheitsreform bricht Europa- und Verfassungsrecht, VersR 2007, S. 431-440.

Böge, Ulf: Der Markt für Krankenhausfusionen aus Sicht des Bundeskartellamts, in: Klauber/Robra/Schellschmidt (Hrsg.), Krankenhausreport 2006 (2007), S. 35-48.

Bohle, Thomas (Hrsg.): Vertragsgestaltung in der Integrierten Versorgung, Landsberg/Lech 2005.

ders.: Die Bedeutung des Kartell- und Wettbewerbsrechts bei Krankenhausfusionen und Krankenhauskooperationen, MedR 2006, S. 259-267. 
Bomba, Wolfgang: Verfassungsmäßigkeit berufs- und standesrechtlicher Werbebeschränkungen für Angehörige freier Berufe (2003).

Braun, Thomas/ Rau, Ferdinand, Tuschen, Karl Heinz: Die DRG-Einführung aus gesundheitspolitischer Sicht. Eine Zwischenbilanz, in: Klauber, Jürgen/ Robra, Bernt-Peter/ Schellschmidt, Henner (Hrsg.), Krankenhaus-Report 2007, Stuttgart 2008, S. 3-22.

Bretthauer, Stefan: Zusammenschlüsse von Krankenhäusern in der Fusionskontrolle, NJW 2006, S. 28842888.

Breuer, Rüdiger: Freiheit des Berufs, in: Isensee, Josef/ Kirchhof, Paul (Hrsg.), Handbuch des Staatsrechts, Bd. VI, § 147, Heidelberg 1989.

Bruckenberger, Ernst: Wettbewerb und Planung, in: Arnold, Michael/ Klauber, Jürgen/ Schellschmidt, Henner (Hrsg.), Krankenhaus-Report 2002, Stuttgart 2003, S. 93-102.

Bruckenberger, Ernst/ Klaue, Siegfried/ Schwintowski, Hans-Peter: Krankenhausmärkte zwischen Regulierung und Wettbewerb, Berlin u.a. 2006.

Bülow, Peter/ Ring, Gerhard: Heilmittelwerbegesetz, Kommentar, 3. Aufl., München 2005.

Bundesversicherungsamt: Wie funktioniert der Risikostrukturausgleich?, abrufbar unter http://www.bundesversicherungsamt.de/Fachinformationen/Risikostrukturausgleich/Informationen /Wie-funktionert-RSA.pdf.

Bultmann, Britta: Die Insolvenzfähigkeit der gesetzlichen Krankenkassen nach dem GKV-OrgWG, MedR 2009, S. 25-32.

Burgi, Martin: Konkurrentenschutz in der Krankenhausplanung, NZS 2005, S. 169-176.

Byok, Jan/ Jansen, Nicola: Die Stellung der gesetzlichen Krankenkassen als öffentlicher Auftraggeber, NVwZ 2005, S. 53-56.

Calliess, Christian /Ruffert, Matthias: EUV/EGV, Kommentar, 3. Aufl., 2007.

Clausen, Tilmann/ Schroeder-Printzen, Jörn: Wahlleistungsvereinbarung/Privatliquidation bei stationären Behandlungen, Heidelberg 2006.

Cremer, Wolfram: Krankenhausfinanzierung im europarechtlichen Kontext, in: Ministerium für Arbeit, Gesundheit und Soziales des Landes Nordrhein-Westfalen (Hrsg.): Krankenhausrecht: Herausforderungen und Chancen, Düsseldorfer Krankenhausrechtstag 2005, S. 23-43.

Dahm, Franz Josef/ Möller, Karl-Heinz/ Ratzel, Rudolf: Rechtshandbuch Medizinische Versorgungszentren, Berlin/ Heidelberg 2005.

Degener-Hencke, Udo: Zum Recht der Krankenhausinvestitionsförderung: Die Baupauschale nach dem nordrhein-westfälischen Krankenhausgestaltungsgesetz vom 11. Dezember 2007, NZS 2009, S. 615.

Deh, Uwe/ Dralle, Ralf: Zentrenbildung im Krankenhaus - ein ungesteuerter Großversuch, in: Klauber, Jürgen/ Robra, Bernt-Peter/ Schellschmidt, Henner (Hrsg.), Krankenhaus-Report 2008/2009, Stuttgart 2009, S. 61-73.

Depenheuer, Otto: Verfassungsrechtliche Grenzen einer Portabilität von Altersrückstellungen in der Krankenversicherung, Gutachten, 2006, erstellt im Auftrag des Verbandes der PKV, abrufbar unter www.pkv.de (Wissenschaft).

Deutsch, Erwin/ Spickhoff, Andreas: Medizinrecht, 6. Aufl., Berlin u.a. 2008.

Deutsches Krankenhausinstitut: Krankenhaus Barometer Umfrage 2005 (2005) bis Umfrage 2008 (2008).

dass.: Krankenhaus Trends, Erhebung Januar 2009. Kurzfristige Investitionsmaßnahmen der Krankenhäuser im Jahr 2009, Düsseldorf 2009.

Dieckman, Thomas J./ Wildberger, Nils/ von Quast, Alexander: Rechtswegzuweisung nach $\S 51$ Abs. 2 SGG bei unlauterem Wettbewerb gesetzlicher Krankenkassen, NZS 2005, S. 187-192. 
Drösler, Saskia E.: Qualitätsberichte gem. § 137 SGB V und ihre Darstellung im Internet - eine vergleichende Analyse, in: Klauber/Robra/Schellschmidt, Krankenhausreport 2006 (2007), S. 207-221.

Ebsen, Ingwer: Perspektiven der Krankenhausplanung in einem gewandelten Markt und einem föderalen Gefüge, in: Klauber, Jürgen /Robra, Bernt-Peter/Schellschmidt, Henner, Krankenhaus-Report 2006 (2007), S. 117-131.

Eggert, Albrecht: Krankenkassen und Kartellamt: Fusionskontrolle zum Schutz des Wettbewerbs in der GKV?, VSSR 2007, S. 335-344.

Ehlers, Alexander P.F./ Sichert, Markus: „Die sozialrechtliche Fortbildungs- und Fortbildungsnachweispflicht der Ärzte“, in: Pharmazeutische Industrie (pharmind) (Vol. 66) Nr. 7/2004, S. 824-826.

Eickmann, Tobias/ Ernst, Johanna: Abgrenzung ambulanter, voll- und teilstationärer Krankenhausbehandlung, KHR 2008, S. 85-88.

Faltin, Jürgen: Freigemeinnützige Krankenhausträger im System staatlicher Krankenhausfinanzierung, Köln u.a. 1996.

Fechner, Frank: Ist das Krankenhausprivileg am Ende? - Vor der Entscheidung des Großen Senats des Bundessozialgerichts (BSG), GesR 2007, S. 355-359.

Fellgiebel, Andreas/ Schanz, Benno/ Huwer, Frank: IV-Vertrag zur Früherkennung von Demenzpatienten mit der Barmer Ersatzkasse, das Krankenhaus 2007, S. 319-324.

Frenz, Walter: Handbuch Europarecht, Band 2, Europäisches Kartellrecht, Berlin u.a. 2006.

ders.: Handbuch Europarecht, Band 3, Beihilfe- und Vergaberecht, Berlin u.a. 2007.

Friedrich, Jörg/ Beivers, Andreas: Patientenwege ins Krankenhaus: Räumliche Mobilität bei Elektiv- und Notfallleistungen am Beispiel von Hüftendoprothesen, in: Klauber, Jürgen/ Robra, Bernt-Peter/ Schellschmidt, Henner (Hrsg.), Krankenhaus-Report 2008/2009, Stuttgart 2009, S. 155-181.

Füsser, Sonja: Das GKV-OrgWG - Gesetz zur Weiterentwicklung der Organisationsstrukturen in der gesetzlichen Krankenversicherung - Inhalt und Motive, SGb 2009, S. 126-131.

Gaßner, Maximilian/ Ahrens, Michaela: Anwendbarkeit der Regeln der Fusionskontrolle des GWB bei der Vereinigung gesetzlicher Krankenkassen, SGb 2007, S. 528-535.

Gent, Andreas: Integrierte Versorgung in der PKV, Präsentation, 1. Hamburger Symposium zur Integrierten Versorgung, 30. November 2005, abrufbar unter http://www.iv-hh.de/iv2006/2005/pdf/Gent.pdf.

Geraedts, Max: Auswirkungen von Qualitätsregulierungen auf das Angebot von Krankenhausleistungen, in: Klauber/Robra/Schellschmidt, Krankenhaus-Reprort 2006 (2007), S. 187-203.

Gerlinger, Thomas: Zwischen Korporatismus und Wettbewerb: Gesundheitspolitische Steuerung im Wandel, Veröffentlichungen der Arbeitsgruppe Public Health, Wissenschaftszentrum Berlin für Sozialforschung 2002.

Göpffarth, Dirk: Der neue Risikostrukturausgleich im Gesundheitsfonds. Wie der morbiditätsorientierte Finanzkraftausgleich funktioniert, Soziale Sicherheit 2009, S. 12-17.

Göpffarth, Dirk/ Sichert, Markus: Morbi-RSA und Einflussnahmen auf ärztliches Kodierverhalten. „Logik“ - Fehlannahmen - Rechtslage, KrV 2009, S. 186-191.

Görs, Benjamin: Krankenhäuser im Wettbewerb: Ausschreibung von Elektivleistungen, KHR 2008, S. 57-61.

Grau, Ulrich/ Dierks, Christian: Die Versorgungsbereiche von öffentlicher und Krankenhausapotheke in der Integrierten Versorgung, A\&R, S. 106-109.

Greß, Stefan/ Ebsen, Ingwer/ Haas, Anne-Katrin/ Schulze, Sabine/ Szecsenyi, Joachim/ Wasem, Jürgen: Wettbewerbliche Steuerung in der GKV - Perspektiven für mehr Qualität und Wirtschaftlichkeit, SF 2003, S. 105-108. 
Greuèl, Marius: Neue Steuerungsansätze im Gesundheitswesen durch Integrierte Versorgung, Betreuungsmanagement 2005, S. 10-14.

Hamann, Erik: Ziele und Strategien der Krankenhausfusionen, Baden-Baden 2000.

Hambüchen, Ulrich: Aktuelle Rechtsprechung des BSG zum Krankenhausrecht, GesR 2008, S. 393-402.

Hansen, Leonhard: Eine empirische Analyse der MVZ im Krankenhaus, in: Klauber, Jürgen/ Robra, Bernt-Peter/ Schellschmidt, Henner (Hrsg.), Krankenhaus-Report 2008/2009, Stuttgart 2009, S. 35-47.

Hauck, Karl/ Noftz, Wolfgang: SGB V, Gesetzliche Krankenversicherung, Kommentar, Bd. 1 und 2, Berlin 2007.

Häussler, Bertram/ Berger, Ursula: Bedingungen für effektive Disease-Management-Programme, BadenBaden 2004.

Hefermehl, Wolfgang/ Köhler, Helmut/ Bornkamm, Joachim: Wettbewerbsrecht, 25. Auflage, München 2007.

Heller, Günther: Gibt es einen Zusammenhang zwischen Menge und Ergebnis bei operativen Routineeingriffen in Deutschland?, in: Klauber, Jürgen/ Robra, Bernt-Peter/ Schellschmidt, Henner (Hrsg.), Krankenhaus-Report 2004, Stuttgart 2005, S. 213-232.

Henke, Klaus-Dirk, Rich, Robert F., Stolte, Hilmar: Integrierte Versorgung und neue Vergütungsformen in Deutschland, Baden-Baden 2004.

Hermann, C./ Hildebrandt, H./ Richter-Reichhelm, M./ Schwartz, F.W./ Witzenrath, W., Managementgesellschaft organisiert Integrierte Versorgung einer definierten Population auf Basis eines - Das Modell „Gesundes Kinzigtal“, GuS 2006, S. 11-29.

Hesselmann, Hildegard/ Motz, Thomas: Integrierte Versorgung und Vergaberecht, MedR 2005, S. 498502.

Höfling, Wolfram: Von Krankenhausrecht zum Krankenhausregulierungsrecht, GesR 2007, S. 289-295.

Isensee, Josef: Wahltarif „Krankenhauskomfort“ - Chefarztbehandlung und Ein-/Zweibettzimmer als Wahlleistungen der Kassen - Sicht des Sozial- und Verfassungsrechts, NZS 2007, S. 449-455.

Isensee, Josef/ Kirchhof, Paul: Handbuch des Staatsrechts der Bundesrepublik Deutschland, Band V, 2. Aufl., München 2000; Band VI, München 1989.

Jarass, Hans D./ Pieroth, Bodo: Grundgesetz, Kommentar, 10. Aufl., München 2009.

Jasper, Jörg/ Sundmacher, Torsten: Wettbewerb und Kooperation als Koordinationsverfahren für die Integrierte Versorgung, SF 2005, S. 53-62.

juris Praxiskommentar SGB V, hrsgg. von Schlegel, Rainer/ Voelzke, Thomas, Saarbrücken, 1. Aufl. 2007.

Kallenberg, Stefan: Der Einheitliche Bewertungsmaßstab (EBM) in der Fassung vom 1.4.2005, GesR 2005, S. 97-102.

Kaltenborn, Markus: Vergaberechtliche Strukturen im Recht der Gesetzlichen Krankenversicherung, VSSR 2006, S. 357-377.

Kamann, Hans-Georg/ Gey, Peter: Wettbewerbsrecht im deutschen Gesundheitswesen - Grenzen der Integrierten Versorgung und der Kooperation von Krankenkassen, Leistungserbringern und pharmazeutischer Industrie, PharmR 2006, S. 255-264; S. 291-297.

Kasseler Kommentar zum Sozialversicherungsrecht, Gesamtredaktion: Klaus Niesel, Band 1, München, Stand: 54. Ergänzungslieferung 2007.

Kies, Friedrich: Der Versorgungsauftrag des Plankrankenhauses, Frankfurt am Main u.a., 1998.

Kingreen, Torsten: Wettbewerbliche Aspekte des GKV-Modernisierungsgesetzes, MedR 2004, S. 188197. 
Knappe, Eckhard (Hrsg.): Wettbewerb in der gesetzlichen Krankenversicherung, Baden-Baden 1999.

Knispel, Ulrich: Doppelter Rechtsweg für Klagen gegen Mitgliederwerbung einer Krankenkasse?, NZS 2008, S. 129-131.

Knorr, Gerhard: Sind PPP und Fördermittel kompatibel? Erfahrungen aus der Sicht der Förderbehörde, das Krankenhaus 2007, S. 793-798.

Knorr, Gerhard/ Kraemer, Alexander: Krankenhausrecht 2005, München 2005.

Koller, Christian: Risikomanagement im Krankenhaus: Haftungsrechtliche Probleme im Rahmen der Integrierten Versorgung nach dem GMG, KHuR 2005, S. 71-74.

ders.: Die Abgabebefugnis von Krankenhausapotheken in der ambulanten Versorgung, ApoR 2006, S. 20-23.

Kölking, Heinz (Hrsg.): DRG und Strukturwandel in der Gesundheitswirtschaft, Stuttgart 2007.

Korenke, Thomas: Innovativer Wettbewerb infolge integrierter Versorgung in der gesetzlichen Krankenversicherung?, SF 2001, S. 268-277.

Krauskopf, Dieter: Soziale Krankenversicherung, Pflegeversicherung, Kommentar, Bd. 1 und 2, München, Stand: März 2007.

Kuchinke, Björn A.: Krankenhausdienstleistungen und Effizienz in Deutschland, Baden-Baden 2004.

Kuhlmann, Jens: Neue Versorgungsmöglichkeiten für Krankenhäuser durch das GMG, das Krankenhaus 2004, S. 13-18.

Kuntz, Ludwig/ Wittland, Michael: Zentrenbildung zur Verbesserung von Qualität und Effizienz - Evidenz am Beispiel der Universitätsklinik Köln, in: Klauber, Jürgen/ Robra, Bernt-Peter/ Schellschmidt, Henner (Hrsg.), Krankenhaus-Report 2008/2009, Stuttgart 2009, S. 87-100.

Laufs, Adolf (Hrsg.)/ Uhlenbruck, Wilhelm (Begründer): Handbuch des Arztrechts, 3. Aufl., München 2002.

Leclerque, Gregor/ Friedrich, Jörg. Die Krankenhausbudgets 2005 bis 2007 unter dem Einfluss der Konvergenz, in: Klauber/Robra/Schellschmidt (Hrsg.), Krankenhaus-Report 2008/2009 (2009), S. 229240.

Lehment, Cornelis: Werberecht für Krankenhäuser und Institute - Grenzüberschreitungen nicht erlaubt, DÄ 2000, S. A 2916-2918.

Lenz, Carl Otto (Hrsg.): EU- und EG-Vertrag, Kommentar, 4. Aufl., Köln u.a. 2006.

Lenz, Christopher/ Dettling, Heinz-Uwe/ Kieser, Timo (Hrsg): Krankenhausrecht, Stuttgart, 2007.

Löcherbach, Peter: Ausbildung zur Case Managerin bzw. zum Case Manager in Deutschland, Vortrag, 4. Augsburger Nachsorgesymposium 2005, abrufbar unter http://www.betainstitut.de/download/ symposium-loecherbach.pdf.

Loewenheim, Ulrich/ Meessen, Karl/ Riesenkampff, Alexander: Kartellrecht, Bd. 1, Europäisches Recht, Kommentar, München 2005.

Loewenheim, Ulrich/ Meessen, Karl/ Riesenkampff, Alexander: Kartellrecht, Bd. 2, GWB, Kommentar, München 2006.

Lübbig, Thomas/ Klasse, Max: Kartellrecht im Pharma- und Gesundheitssektor, Baden-Baden 2007.

Lüttike, Jürgen/ Schellschmidt, Henner: Qualitätsberichte nach § 137 SGB V - Bewertung und Vorschläge zur Erweiterung, in: Klauber, Jürgen/ Robra, Bernt-Peter/ Schellschmidt, Henner (Hrsg.), Krankenhaus-Report 2004, Stuttgart 2005, S. 197-211.

von Mangoldt, Hermann/ Klein, Friedrich/ Starck, Christian (Hrsg.): Das Bonner Grundgesetz, Kommentar, Bd. 1 und 2, 5. Aufl., München 2005.

Mayntz, Renate/ Derlien, Hans-Ulrich: Die Organisation der gesetzlichen Krankenversicherung, - eine strukturell-funktionale Problemstudie, Bonn 1979. 
Mau, Harald: Integrierte Versorgung - Chancen und Risiken aus der Sicht der klinischen Praxis, KHuR 2004, S. 71-75.

Mickwitz, Elena von: Organisation und Haftung vernetzter Kooperationsformen in der gesetzlichen Krankenversicherung, Baden-Baden 2006.

Möller, Ralf: Kartellrechtliche Kontrolle des Krankenhaussektors, das Krankenhaus 2007, S. 313-312.

Monopolkommission: Weniger Staat, Mehr Wettbewerb - Gesundheitsmärkte und staatliche Beihilfen in der Wettbewerbsordnung, Siebzehntes Hauptgutachten der Monopolkommission gemäß $§ 44$ Abs. 1 Satz 1 GWB (2006/2007) 2008, BT-Drs. 16/10140.

Mühlhausen, Mitgliederwettbewerb innerhalb der gesetzlichen Krankenversicherung, Baden-Baden 2002.

Müller, Matthias: Krankenhauswerbung - Möglichkeiten und rechtliche Grenzen, Pflege \& Krankenhausrecht 2006, S. 88-91.

Neubauer, Günter: Zur Zukunft der dualen Finanzierung unter Wettbewerbsbedingungen, in: Arnold, Michael/ Klauber, Jürgen/ Schellschmidt, Henner (Hrsg.), Krankenhaus-Report 2002, Stuttgart 2003, S. 71-91.

ders.: Zur ökonomischen Steuerung der Krankenhausversorgung unter DRG-Fallpauschalen, in: Klauber, Jürgen/ Robra, Bernt-Peter/ Schellschmidt, Henner (Hrsg.), Krankenhaus-Report 2003, Stuttgart 2004, S. 101-119.

ders./ Beivers, Andreas/ Minartz, Christof: Marktwandel und Sicherstellung der regionalen Krankenhausversorgung, in: Klauber, Jürgen/ Robra, Bernt-Peter/ Schellschmidt, Henner (Hrsg.), KrankenhausReprort 2006 (2007), S. 65-85.

Orlowski, Ulrich/ Rau, Ferdinand/ Schermer, Joachim/ Wasem, Jürgen/ Zipperer, Manfred (Hrsg.), GKVKommentar, SGB V, Bd. 1 und 2, Heidelberg, Stand: August 2007.

Otto, Frank/ Göpffarth, Dirk: RSA-Jahresausgleich 2006 - Daten und Fakten, in: Göpffarth, Dirk u.a. (Hrsg.), Jahrbuch Risikostrukturausgleich 2008, S. 303-350.

Patt, Joachim: System und Kontrolle der Preisbildung für Krankenhausleistungen, Baden-Baden 1996.

Pester, Rüdiger: Die wettbewerblichen Rechtsbeziehungen im Mitgliederwettbewerb der gesetzlichen Krankenkassen, Hamburg 2005.

Peters, Horst: Handbuch der Krankenversicherung Teil II - Sozialgesetzbuch V, Bd. 2 und 3, Stuttgart, Stand: 65. Ergänzungslieferung (Juli 2007).

Pfaff, Martin/ Wassener, Dietmar: Das Krankenhaus im Gefolge des Gesundheits-Struktur-Gesetzes 1993, Baden-Baden 1995.

Pfohl, Andreas: Die Neuregelung der Finanzierungsströme durch den Gesundheitsfonds, in. Göpffarth, Dirk u.a. (Hrsg.) Jahrbuch Risikostrukturausgleich 2007 (2007), S. 195-214.

Pfohl, Andreas/ Sichert, Markus: Der Gesundheitsfonds: Sondervermögen des Bundes oder der Krankenkassen?, in: NZS 2009, S. 71-79.

Pinter, Erwig: Qualitätssicherung im Krankennhaus nach dem GKV-WSG, KHR 2008, S. 1-6.

Pouvourville, Gérard de: Kann Qualität ein Wettbewerbsparameter im Gesundheitswesen sein?, in: Arnold, Michael/ Klauber, Jürgen/ Schellschmidt, Henner (Hrsg.), Krankenhaus-Report 2002, Stuttgart 2003, S. 175-188.

Prölls, Jürgen/ Martin, Anton: Versicherungsvertragsgesetz, Kommentar, 27. Aufl., München 2004.

Quaas, Michael: Vertragsgestaltungen zur integrierten Versorgung aus der Sicht der Krankenhäuser, VSSR 2004, S.175-195.

ders.: Sind Wahlleistungen in Privatkliniken eine finanzierungsrechtliche Alternative?, KHuR 2006, S. 114-121.

ders./ Zuck, Rüdiger: Medizinrecht, 2. Aufl., München 2008. 
Ratzel, Rudolf/ Lippert, Hans-Dieter, Kommentar zur Musterberufsordnung der deutschen Ärzte (MBO), 4. Aufl., Berlin u.a. 2006.

Rau, Ferdinand, Regelungen des Krankenhausfinanzierungsreformgesetzes, das Krankenhaus 2009, S. 198-208.

Rechenberg, Christian von: Case-Manager: Wenn die Schwester den Arzt ersetzt, abrufbar unter http://morgenmagazin.zdf.de/ZDFde/inhalt/17/0,1872,7011985,00.html.

Regorz, Arndt: Vertragswettbewerb in der Krankenhausversorgung - Auswirkungen auf den Kassenwettbewerb, KHR 2008, S. 66-69.

Reischl, Klaus: Insolvenzrecht, Heidelberg 2008.

Rengeling, Hans-Werner/ Middeke, Andreas/ Gellermann, Martin: Handbuch des Rechtsschutzes in der Europäischen Union, 2. Aufl., München 2003.

Richter-Reichhelm, Manfred: Faire Wettbewerbschancen für alle, Forum für Gesundheitspolitik 2004, S. 165-169.

Rixen, Stephan: Vergaberecht oder Sozialrecht in der gesetzlichen Krankenversicherung? - Ausschreibungspflichten von Krankenkassen und Kassenärztlichen Vereinigungen, GesR 2006, S. 49-58.

Robbers, Boris: Geplanter Wettbewerb. Mögliche Auswirkungen von Monistik und Einzelverträgen auf die Flächenversorgung, das Krankenhaus 2007, S. 207-210.

Rolfs, Christian/ Kreikebohm, Ralf/ Giessen, Richard/ Udsching, Peter (Hrsg.): Beck'scher OnlineKommentar Sozialrecht, Edition 13, München 2009.

Römer, Wolfgang/ Langheid, Theo: Versicherungsvertragsgesetz, Kommentar, 2. Aufl., München 2003.

Roth, Wulf-Henning: Kartellrechtliche Aspekte der Gesundheitsreform nach deutschem und europäischem Recht, GRUR 2007, S. 645-659.

Rüffer, Wilfried/ Halbach, Dirk/ Schimikowski, Peter: Versicherungsvertragsgesetz, Handkommentar, Baden-Baden, 2009.

Rüfner, Wolfgang: Grundrechtsträger, in: Isensee, Josef/ Kirchhof, Paul (Hrsg.), Handbuch des Staatsrechts, Bd. V, § 116, Heidelberg, 2. Aufl. 2000.

Sachverständigenrat zur Begutachtung der Entwicklung im Gesundheitswesen, Kooperation und Verantwortung, Voraussetzungen einer zielorientierten Gesundheitsversorgung, Gutachten 2007, abrufbar unter http://www.svr-gesundheit.de/Startseite/Startseite.htm.

Scharpf, Fritz/ Reissert, Bernd/ Schnabel, Fritz: Politikverflechtung: Theorie und Empirie es kooperativen Föderalismus in der Bundesrepublik, Kronberg 1976.

Schmehl, Arndt/ Wallrabenstein, Astrid (Hrsg): Steuerungsinstrumente im Recht des Gesundheitswesens, Bd. 1, Wettbewerb, Tübingen 2005.

Schmidt, Dorothee/ Wittmann, Rüdiger, Göpffarth, Dirk: Morbiditätsorientierte Datenmeldungen für die Weiterentwicklung des Risikostrukturausgleichs, in: Göpffarth u.a. (Hrsg.), Jahrbuch Risikostrukturausgleich 2008, S. 275-302.

Schmidt, Tillman: GKV-WSG: die Relativierung klassischer Sozialversicherungsmerkmals in der GKV durch veränderte Beitragsbemessung und Wahltarife, GesR 2007, S. 295-302.

Schnapp, Friedrich/ Wigge, Peter (Hrsg.): Handbuch des Vertragsarztrechts, 2. Aufl., München 2006.

Schrappe, Matthias: Qualitätstransparenz - Qualitätsmanagement und Qualität im Wettbewerb: Klauber, Jürgen/ Robra, Bernt-Peter/ Schellschmidt, Henner (Hrsg.), Krankenhaus-Report 2004, Stuttgart 2005, S. 17-26.

Schwarze, Jürgen (Hrsg.): EU-Kommentar, 2. Aufl., Baden-Baden 2009.

Seeringer, Stefanie: Der Gemeinsame Bundesausschuß nach dem SGB V, Baden-Baden 2006. 
Sichert, Markus: Abkehr vom Kollektivvertragssystem in der integrierten Versorgung: Verbot und Folgen der Beteiligung Kassenärztlicher Vereinigungen, VSSR 2006, S. 272-299.

ders.: Constitutional Review of Social Laws in Germany and its Impact on Social Policy and LawMaking, Zbnornik Pravnog fakulteta Sveučilišta u Rijeci, Vol. 27 (2006) no. 2, S. 725-759.

ders.: Neue Versorgungsformen und Rabattregelungen für ausländische Leistungserbringer, in: Schwarze, Jürgen/ Becker, Ulrich (Hrsg.), Arzneimittel im Europäischen Binnenmarkt, EuR, Beiheft 2/2007, S. 101-144.

ders.: Die Rolle des europäischen Rechts für die Zukunft des deutschen Gesundheitswesens, Die BKK 2007, S. 396-400.

ders.: Gemeinschaftsrechtliche Vorgaben für Integrierte Versorgung, GuS, Heft 11/2007.

Sichert, Markus/ Walser, Christina: The Management of Chronic Diseases in The Netherlands and in Germany, EJSS 3/2007, S. 243-275.

Sodan, Helge: Private Krankenversicherung und Gesundheitsreform 2007, Berlin 2007.

Steinbrück, Ralph: Medizinische Versorgungszentren - Chancen und Risiken für Vertragsärzte und Krankenhäuser!, KHuR 2005, S. 1-6.

Steiner, Peter/ Bussmann, Jens/ Koerdt, Stefan: „Vereinbarung zum Fallpauschalensystem für Krankenhäuser (FPV 2007) - Abrechnung nach der PFV 2007, Redaktionsbeilage/FPV 2007, in: das Krankenhaus 1/2007.

Steinmeyer, Heinz-Dietrich: Wettbewerbsrecht im Gesundheitswesen, Berlin 2000.

Stollmann, Frank: Mindestmengenregelung nach $§ 137$ SGB V - Ausnahmeentscheidung der Planungsbehörde, GesR 2007, S. 303-309.

Strehl, Rüdiger: Privatisierungswelle im deutschen Krankenhauswesen?, in: Arnold, Michael/ Klauber, Jürgen/ Schellschmidt, Henner (Hrsg.), Krankenhaus-Report 2002, Stuttgart 2003, S. 113-129.

Streinz, Rudolf (Hrsg.): EGV/EUV, München 2003.

Tuschen, Karl Heinz/ Trefz, Ulrich: Krankenhausentgeltgesetz, 1. Aufl., Stuttgart 2004.

Uleer, Christoph/ Miebach, Jürgen/ Patt, Joachim: Abrechnung von Arzt- und Krankenhausleistungen, Kommentar, München 2006.

Veit, Christof, Qualitätssicherung im Krankenhaus: Verbesserte Ergebnisse - weiterhin Handlungsbedarf, ErsK 2008, S. 423-425.

Verband der privaten Krankenversicherung e.V. (Hrsg.): Zahlenberichte der privaten Krankenversicherung 2003/2004 bis 2007/2008, Köln 2004 bis 2008.

Vollmöller, Thomas: Rechtsfragen bei der Umsetzung von Disease-Management-Programmen, NZS 2004, S. 63-68.

ders.: Die Schnittstelle Krankenhaus - Rehabilitation, ZMGR 2006, S. 171-177.

ders.: Krankenhausfinanzierung und EG-Beihilfenrecht, in: Bauer u.a. (Hrsg.), Wirtschaft im offenen Verfassungsstaat, Festschrift für Reiner Schmidt, München, 2006, S. 205-214.

ders.: Die Vereinbarkeit der Öffnung der Krankenhäuser für ambulante Leistungen (§ 116b II SGB V) mit der Berufsfreiheit der niedergelassenen Vertragsärzte (Art. 12 I GG), NZW 2006, S. 572-578.

Wagner, Karin/ Lenz, Immo (Hrsg.): Erfolgreiche Wege in die Integrierte Versorgung, Stuttgart 2007.

Wallhäuser, Matthias: Verträge in der Integrierten Versorgung, Heidelberg 2005.

Walter, Ute: Pauschalentgelte von Krankenhäusern an niedergelassenen Ärzte - zulässige Zuweiserbindung oder berufsrechtswidrige Provision?, KHUR 2006, S. 71-77.

Waltermann, Raimund: Sozialrecht, 6. Aufl., Heidelberg 2006. 
Wasem, Jürgen/Rotter, Isabelle/ Focke/Axel, Igl, Gerhard: Verändertes Kodierverhalten als eine der ungeklärten Herausforderungen der DRG-Einführung, in: Klauber, Jürgen/ Robra, Bernt-Peter/ Schellschmidt, Henner (Hrsg.), Krankenhaus-Report 2007, Stuttgart 2007, S. 49-59.

Weatherly, John N. u.a., Leuchtturmprojekte Integrierter Versorgung und Medizinischer Versorgungszentren. Innovative Modelle in der Praxis, Berlin 2007.

Welmer, Tobias: Klinikwerberecht - Marketing und Strategie, Pflege \& Krankenhausrecht 2005, S. 7376.

Wenner, Ulrich: Einbeziehung von Krankenhäusern in die ambulante ärztliche Versorgung - Auswirkungen des Vertragsarztrechtsänderungsgesetzes (VÄndG) und des GKV-Wettbewerbsstärkungsgesetzes (GKV-WSG), GesR 2007, S. 337-246.

Wenzel, Frank (Hrsg.): Handbuch des Fachanwalts Medizinrecht, Köln 2007.

Wigge, Peter: Medizinische Versorgungszentren nach dem GMG, MedR 2004, S. 123-134.

Wille, Eberhard (Hrsg.): Zur Rolle des Wettbewerbs in der Gesetzlichen Krankenversicherung, BadenBaden 1999.

Winterer, Arndt: Neue Wege der Krankenhausfinanzierung - leistungsbezogene Investitionsförderung in NRW, in: Klauber, Jürgen/ Robra, Bernt-Peter/ Schellschmidt, Henner (Hrsg.), KrankenhausReport 2008/2009 (2009), Stuttgart 2009, S. 143-154.

Wolf, Sven: Der neue Zusatzbeitrag nach $\S 242$ SGB V, GesR 2008, S. 567-573.

Zwingel, Bernd/ Preißler, Reinhold: Das Medizinische Versorgungszentrum, Köln 2005. 


\title{
Wahlmöglichkeiten und Wettbewerb in der Krankenhausversorgung aus gesundheitsökonomischer Sicht - Kommentar zum Bericht über Deutschland
}

\author{
Günter Neubauer
}

1. Das deutsche Krankenhausversorgungssystem als planwirtschaftlicher Entwurf 264

2. Transformation von der Planwirtschaft zu einer Wettbewerbswirtschaft 266

3. Ist freier Wettbewerb für die Krankenhausversorgung zielführend? 266

4. Krankenhausversorgung und Wettbewerbsrecht 268

5. Zweiteilung des Vertragsmarktes durch kollektive und selektive Verträge 269

6. Ausblick: Das Krankenhausfinanzierungsreformgesetz von 2008/9 272 